\title{
Synthesis and Analysis of Modified SNARE Proteins with Respect to Assembly and Disassembly of the SNARE Complex
}

\author{
Dissertation \\ for the award of the degree \\ "Doctor rerum naturalium" \\ of the Georg-August-Universität Göttingen \\ within the doctoral program \\ International Max-Planck Research School \\ "Physics of Biological and Complex Systems" \\ of the Georg-August University School of Science (GAUSS) \\ submitted by \\ Meike Pauline Wilhelmine Junius, née Quast \\ from Lemgo
}

Göttingen 2016 


\section{Thesis Committee}

Prof. Dr. Ulf Diederichsen

(Institute of Organic and Biomolecular Chemistry, Georg-August-University Göttingen)

Prof. Dr. Reinhard Jahn

(Department of Neurobiology, Max-Planck-Institute for biophysical Chemistry Göttingen)

Prof. Dr. Claudia Steinem

(Institute of Organic and Biomolecular Chemistry, Georg-August-University Göttingen)

\section{Members of Examination Board}

Referee:

Prof. Dr. UIf Diederichsen (Institute of Organic and Biomolecular Chemistry, Georg-AugustUniversity Göttingen)

$2^{\text {nd }}$ Referee:

Prof. Dr. Reinhard Jahn (Department of Neurobiology, Max-Planck-Institute for biophysical Chemistry Göttingen)

\section{Further members of the Examination Bord}

Prof. Dr. Claudia Steinem (Institute of Organic and Biomolecular Chemistry, Georg-AugustUniversity Göttingen)

Prof. Dr. Claudia Höbartner (Institute of Organic and Biomolecular Chemistry, Georg-AugustUniversity Göttingen)

Dr. Franziska Thomas (Institute of Organic and Biomolecular Chemistry, Georg-AugustUniversity Göttingen)

Dr. Alexander Breder (Institute of Organic and Biomolecular Chemistry, Georg-August-University Göttingen)

Date of oral examination: 26.08 .2016 
This work was supported by the Deutsche Forschungsgemeinschaft via the Collaborative Research Center 803 (SFB 803) "Functionality controlled by organization in and between membranes" and has been carried out under the supervision of Prof. Dr. Ulf Diederichsen at the Institute of Organic and Biomolecular Chemistry of the Georg-August-University Göttingen between July 2012 and June 2016.

I like to thank Prof. Dr. Ulf Diederichsen for the opportunity to work on an interesting and highly collaborative research topic within his group, for his support and guidance as well as for the freedom of research. 


\section{Table of Contents}

\begin{tabular}{lll}
\hline 1 & Introduction & 1
\end{tabular}

2 SNARE-Mediated Membrane Fusion $\quad \mathbf{5}$

2.1 Synaptic Transmission . . . . . . . . . . . . . . . . . . . . . . . . . . . . . 6

2.2 SNARE Proteins and SNARE Complex . . . . . . . . . . . . . . . . . 7

2.3 A Closer Look at SNARE Protein Synaptobrevin-2 . . . . . . . . . . . . . . . . . . 10

2.4 The Cycle of SNARE-Mediated Membrane Fusion $\ldots \ldots \ldots$. . . . . . . . . . . . . 12

2.5 Priming and Assembly of the SNARE Complex . . . . . . . . . . . . . . . . 13

2.6 The NSF $/ \alpha$-SNAP-Mediated SNARE Disassembly Reaction . . . . . . . . . . . 16

$2.6 .1 \quad N$-ethylmaleimide-sensitive factor (NSF) $\ldots \ldots \ldots$

2.6 .2 Soluble $N$-Ethylmaleimide-Sensitive Factor Attachment Proteins (SNAPs) 19

2.6 .3 Structure of the $20 \mathrm{~S}$ (super)complex . . . . . . . . . . . . . 20

2.6 .4 Mechanism of NSF $/ \alpha$-SNAP-Mediated SNARE Disassembly . . . . . . 24

2.7 Complexin - A Regulatory Protein of SNARE-Mediated Membrane Fusion . . . 24

2.7 .1 Structure of the Complexin/SNARE Complex . . . . . . . . . . . . . . 26

2.7 .2 Controversially Discussed Function of Complexin . . . . . . . . . . . . 26

2.7 .3 Collaborative Regulation by Complexin and Synaptotagmin . . . . . . 31

2.7 .4 Does Complexin Compete with $\alpha$-SNAP? . . . . . . . . . . . . . . . . 34

3 Photocleavable Protecting Groups and Their Applications 37

3.1 General Design of PPGs and Caged Peptides/Proteins . . . . . . . . . . . . . 37

3.2 Instrumentations for Photocleavage Reactions . . . . . . . . . . . . . . . . . 38

3.3 Most Frequently Used Photocleavable Protecting Groups . . . . . . . . . . . . . . 40

3.3.1 0 o-Nitrobenzyl Groups . . . . . . . . . . . . . . . . . . . . . . . . . . 40

3.3 .2 Coumarin-4-ylmethyl Groups . . . . . . . . . . . . . . . . . . . . . . . 41

3.4 Two-Photon Uncaging . . . . . . . . . . . . . . . . . . . . . . . . . . . . . . . . . . 42

3.5 Wavelength-Selective Photocleavage . . . . . . . . . . . . . . 43

3.6 Biological Applications of PPGs in Neuroscience . . . . . . . . . . . . . . . . 45

4 Synthesis and Analyses of Adamantyl-Modified Synaptobrevin Analogues 47

4.1 The Interaction Between $\alpha$-SNAP and SNARE Complex . . . . . . . . . . . . 47

4.2 Solid-Phase Peptide Synthesis (SPPS) . . . . . . . . . . . . . . . . . . . . . . . . . . . 49 
4.3 Synthesis of Adamantyl-Modified Synaptobrevin Analogues . . . . . . . . . . . . 51

4.3.1 Synthesis of N-Fmoc Adamantyl Amino Acid Building Block (4). . . . 52

4.3.2 Synthesis of Adamantyl-Modified Synaptobrevin-2 (28-84) . . . . . . 53

4.4 Fluorescence Anisotropy . . . . . . . . . . . . . . . . . . . . . . . . . . 54

4.5 SNARE Assembly and Disassembly Properties of Adamantyl-Modified Synaptobrevin Analogues . . . . . . . . . . . . . . . . . . . . . . . . . . . . . . 57

4.5.1 SNARE Complex Formation and Reconstitution into SUVs and CoFloatation Assay . . . . . . . . . . . . . . . . . . . . . 57

4.5 .2 Fluorescence Anisotropy Experiments of SNARE Complex Formation . 59

4.5 .3 Fluorescence Anisotropy Experiments of SNARE Disassembly Reaction 61

4.6 Complexin Binding Studies with Adamantyl-Modified Synaptobrevin Analogues 63

4.6.1 Fluorescence Anisotropy Experiments of Complexin Binding - Proof of Principle . . . . . . . . . . . . . . . . . . . . . 63

4.6.2 Complexin Binding to SNARE Complexes Containing Syb-2 (28 - 84) Wild-Type (6) . . . . . . . . . . . . . . . . 65

4.6.3 Complexin Binding to SNARE Complexes Containing Adamantyl-Modified Synaptobrevin-2 Analogues . . . . . . . . . . . . . . . . 66

4.7 Conclusion $\ldots \ldots \ldots \ldots$. . . . . . . . . . . . . . . . . . . . . . . . . . . . . . . . . . . . . . . . . . . 72

5 Synthesis and Impact on SNARE Zippering of a Soluble Caged Synaptobrevin2 Analogue $\quad 75$

5.1 SNARE Zippering Studied by Synaptobrevin-2 Mutants. . . . . . . . . . . . . 76

5.2 Design and Synthesis of Soluble Caged Synaptobrevin-2 . . . . . . . . . . . . 79

5.2 .1 Synthesis of Photocleavable Amino Acid Building Blocks . . . . . . . . 81

5.2 .2 Synthesis and Photocleavage of Caged Test Peptides 28 - 31 . . . . . 84

5.2 .3 Synthesis Strategy and Photocleavage of Soluble Caged Synaptobrevin-2

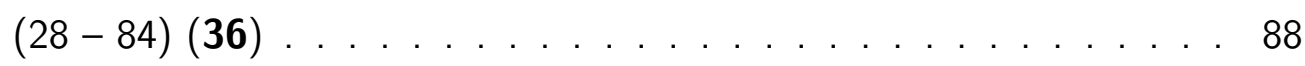

5.3 Binding Studies of Soluble Caged Synaptobrevin-2 $(28-84)$. . . . . . . . . . . 90

5.4 Conclusion . . . . . . . . . . . . . . . . . . . . . . . . . . . . . . . . . . . . . . 91

6 Synthesis of Labelled Syntaxin Transmembrane Domains for Investigations of $\begin{array}{ll}\text { Syntaxin Clustering } & 95\end{array}$

6.1 Synthesis of Labelled Syntaxin TMDs . . . . . . . . . . . . . . . . . . . . . . . . . 95

6.1 .1 Labelling reaction with Atto647N . . . . . . . . . . . . . . . . . . . 96

6.1 .2 Labelling reaction with Rhodamine Red . . . . . . . . . . . . . . . . . 96

6.1 .3 Labelling Reaction with Oregon Green . . . . . . . . . . . . . . . . . 97

6.1 .4 Labelled Syntaxin Transmembrane Domains. . . . . . . . . . . . . . . 98

6.2 Synthesis of Labelled VAMP TMDs $\ldots \ldots \ldots$

6.3 Application of Labelled Syntaxin TMDs . . . . . . . . . . . . . . . . . . . . . . . . . . . . . 99 
\begin{tabular}{lll}
\hline 7 & Summary & 101
\end{tabular}

8 Experimental Part 105

8.1 General Synthetic Methods and Materials . . . . . . . . . . . . . . . . . 105

8.2 Characterisation . . . . . . . . . . . . . . . . . . . . . . . . . . . 106

8.3 Analytical and Spectroscopic Methods . . . . . . . . . . . . . . . . . . . 107

8.3 .1 UV/Vis Spectroscopy . . . . . . . . . . . . . . . . . . . . . . . . 107

8.3 .2 Bradford Assay . . . . . . . . . . . . . . . . . . . . . . . . . . 107

8.3 .3 BCA Assay . . . . . . . . . . . . . . . . . . . . . . . . . . 107

8.3 .4 Photocleavage . . . . . . . . . . . . . . . . . . . . . . . 108

8.3.5 SNARE Protein Constructs, Expression and Purification . . . . . . . . 108

8.3 .6 SNARE Complex Assembly . . . . . . . . . . . . . . . . . . . . . . 108

8.3.7 Reconstitution of SNAREs into Small Liposomes . . . . . . . . . . . 110

8.3 .8 Co-floatation Assay . . . . . . . . . . . . . . . . . . . . . . . . 111

8.3.9 Fluorescence Anisotropy Experiments . . . . . . . . . . . . . . . . . . 111

8.4 General Synthesis Procedures (GSP) $\ldots \ldots$. . . . . . . . . . . . . . . . 112

8.4 .1 GSP 1: Manual Solid Phase Peptide Synthesis . . . . . . . . . . . . 113

8.4.2 GSP 2: Automated Solid Phase Peptide Synthesis . . . . . . . . . . 113

8.4 .3 GSP 3: Peptide Cleavage from Solid-Support . . . . . . . . . . . . . . . 114

8.4 .4 GSP 4: KAISER Test . . . . . . . . . . . . . . . . . . . . . . . . . . . . . 115

8.5 Synthesis of Adamantyl-Modified Amino Acid Building Block (4) . . . . . . . 116

8.6 Adamantyl modified Synaptobrevin-2 analogues . . . . . . . . . . . . . . . . . 120

8.6 .1 Synthesis of Synaptobrevin-2 (66 - 84) (5) . . . . . . . . . . . . . . 120

8.6 .2 Synthesis of Synaptobrevin-2 $(28-84)(6)$. . . . . . . . . . . . . . . 121

8.6.3 Synthesis of Synaptobrevin-2 (28 - 84) E55N(Ad), D65N(Ad) (7) . . . 123

8.6.4 Synthesis of Synaptobrevin-2 (28 - 84) D51N(Ad), E55N(Ad) (8) . . . 124

8.6 .5 Synthesis of Synaptobrevin-2 (28 - 84) D51N(Ad), E55N(Ad), D65N(Ad)

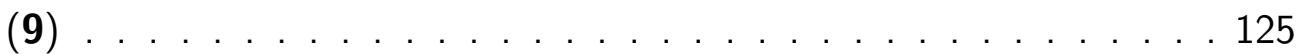

8.6 .6 Synthesis of Synaptobrevin-2 (28 - 84) Q58N(Ad) (10) . . . . . . . . 126

8.6.7 Synthesis of Synaptobrevin-2 (28 - 84) E62N(Ad) (11) . . . . . . . . 127

8.7 Photocleavable Amino Acid Building Blocks $\ldots \ldots$. . . . . . . . . . . . . 128

8.7.1 Synthesis of caged lysine $N$-Fmoc buidling block (16) . . . . . . . . . 128

8.7.2 Synthesis of caged arginine $N$-Fmoc building block (21) . . . . . . . . 131

8.7.3 Synthesis of caged aspartic acid N-Fmoc building block (27) . . . . . 136

8.8 Caged Synaptobrevin-2 analogues . . . . . . . . . . . . . . . . . . . . . . . . 145

8.8.1 Synthesis of Test Peptides 28 - 33 . . . . . . . . . . . . . . . . . . 145

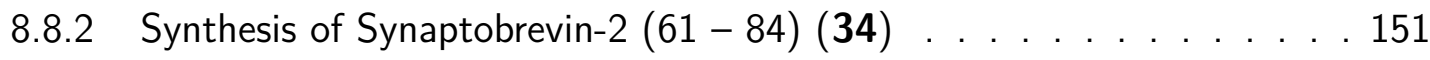

8.8.3 Synthesis of Synaptobrevin-2 (51 - 84) K52K(Nvoc), D57D(DMNPB), K59K(Nvoc) (35) . . . . . . . . . . . . . . . . . . . . . 152 
8.8.4 Synthesis of Synaptobrevin-2 (28 - 84) K52K(Nvoc), D57D(DMNPB), K59K(Nvoc) (36) . . . . . . . . . . . . . . . . . . . 153

8.9 Labelled SNARE transmembrane domains . . . . . . . . . . . . . . . . . . . . 154

8.9.1 Synthesis of Syntaxin-4 (262 - 297) Homo sapiens (37) . . . . . . . . 154

8.9 .2 Synthesis of Syntaxin-1A (254 - 288) Homo sapiens (38) . . . . . . . 155

8.9.3 Synthesis of Syntaxin-1A (254 - 288) K264A, K265A Homo sapiens (39)156

8.9.4 Synthesis of Syntaxin-1A (257 - 288) M267A, C271A, I279A Rattus norvegicus (40) . . . . . . . . . . . . . . . . . . . . . 157

8.9.5 Synthesis of Atto647N-labelled Syntaxin-4 262 - 297 Homo sapiens (41) 158

8.9.6 Synthesis of Atto647N-labelled Syntaxin-1A (254 - 288) Homo sapiens (42)

8.9.7 Synthesis of Atto647N-labelled Syntaxin-1A (254 - 288) K264A, K265A Homo sapiens (43) . . . . . . . . . . . . . . . . . . . . . . . . . . . 160

8.9.8 Synthesis of Atto647N-labelled Syntaxin-1A (257 - 288) M267A, C271A, I279A Rattus norvegicus (44) . . . . . . . . . . . . . . . . . . . . 161

8.9.9 Synthesis of RhodamineRed-labelled Syntaxin-4 (262 - 297) Homo sapiens (45) . . . . . . . . . . . . . . . . . . . . . . . . . . . . . 162

8.9.10 Synthesis of RhodamineRed-labelled Syntaxin-1A (254 - 288) Homo sapiens (46) . . . . . . . . . . . . . . . . . . . . . . . . 163

8.9.11 Synthesis of RhodamineRed-labelled Syntaxin-1A (257 - 288) M267A, C271A, I279A Rattus norvegicus (47) . . . . . . . . . . . . . . . . . 164

8.9.12 Synthesis of OregonGreen 488-labelled Syntaxin-1A (254 - 288) Homo sapiens (48) . . . . . . . . . . . . . . . . . . . . . . . . . . . 165

8.9.13 Synthesis of OregonGreen 488-labelled Syntaxin-1A (257 - 288) M267A, C271A, I279A Rattus norvegicus (49) . . . . . . . . . . . . . . . . . 166

8.9.14 Synthesis of VAMP-3 $(70-100)+C V S$ Rattus norvegicus (50) . . . . 167

8.9.15 Synthesis of VAMP-8 (63 - 100) Rattus norvegicus (51) . . . . . . . . . 168

8.9 .16 Synthesis of hybrid construct VAMPext (52) . . . . . . . . . . . . . . 169

8.9.17 Synthesis of Atto647N-labelled VAMP-3 (70 - 100)+CVS Rattus norvegicus (53) . . . . . . . . . . . . . . . . . . . . . . . . . . 170

8.9.18 Synthesis of Atto647N-labelled VAMP-8 (63 - 100) Rattus norvegicus

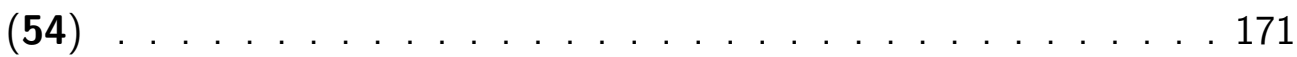

8.9.19 Synthesis of Atto647N-labelled hybrid construct VAMPext (55) . . . . 172

8.9.20 Synthesis of RhodamineRed-labelled VAMP-3 (70 - 100)+CVS Rattus norvegicus (56) . . . . . . . . . . . . . . . . . . . . 173

8.9.21 Synthesis of RhodamineRed-labelled VAMP-8 (63 - 100) Rattus norvegicus (57) . . . . . . . . . . . . . . . . . . . . . . . . . . . . . . 174 
\begin{tabular}{lr}
\hline Bibliography & 180
\end{tabular}

\begin{tabular}{lr}
\hline List of Figures & 189
\end{tabular}

\begin{tabular}{ll}
\hline Acknowledgement & 195
\end{tabular} 


\section{Introduction}

During the evolutionary process, development of eukaryotic cells and their membrane-enclosed organelles enabled the reaction separation within the cells as well as reaction localisation on membrane surfaces. The impermeability of the lipid bilayers towards most hydrophilic molecules brought these advantages, but for maintaining the communication within and between the cells, certain mechanisms to specifically overcome the barrier were needed. One transport possibility through the lipid bilayer are transport vesicles filled with cargo molecules. The vesicles are loaded by membrane fission or endocytosis in which the membrane is everted, the corresponding cargo is surrounded, and subsequently clamped from the source membrane. As soon as the target membrane is reached, the cargo molecules are released from the vesicle by membrane fusion or exocytosis. The two opposing membranes of vesicle and target compartment are pulled in close proximity and water molecules are excluded to enable the fusion process. A machinery of specialised proteins are necessary for each of these basic mechanisms to enable cell communication and nutrition by transport vesicles. [1 2]

Several life essential events are depending on endo- and excocytosis, for example immune reactions, propagation, and synaptic transmission. For many of these processes, membrane fission and fusion are executed and regulated by their own set of specialised proteins. One example is the family of soluble $N$-ethylmaleimide-sensitive factor attachment protein receptor (SNARE) proteins which mediate the fusion of transport vesicles along the secretory pathway of the cells. By anchorage in the opposing membranes and formation of a highly stable coiled coil, SNARE proteins pull the membranes in close proximity and provide a certain amount of energy required for fusion. [3 4] First identified as targets of neurotoxins, 3] 4] SNARE proteins were especially found to mediate neurotransmitter release at presynaptic nerve terminals. $3-7.7$

Within the central nervous system, the electrical signals are transferred between nerve cells via the synaptic cleft, but to overcome this interface, the electrical signal needs to be translated into a chemical signal. For this purpose, neurotransmitters are stored in synaptic vesicles at the presynaptic nerve terminal. Upon stimulation, the membrane fusion process is triggered by $\mathrm{Ca}^{2+}$ influx, the neurotransmitters are released in the synaptic cleft and the signal is thereby transferred to the postsynaptic nerve terminal. Subsequent binding of the neurotransmitters to receptors triggers again an electrical signal within the postsynaptic nerve cell.11 2] Dysfunctions of this process cause neuronal and/or neurodegenerative diseases and therefore the process 


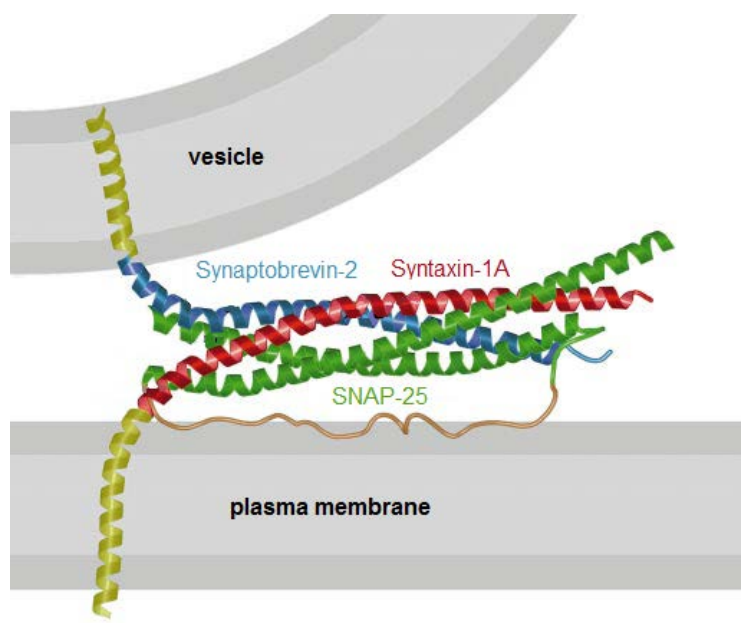

Figure 1.1: Model of neuronal SNARE complex resided between synaptic vesicle and plasma membrane. Synaptobrevin-2 (blue) and Syntaxin-1A (red) are anchored in the membranes by transmembrane domains (TMD, yellow). The anchorage of SNAP-25 (green) occured by palmitoylated Cys residues within its linker region (beige). Figure modified from SUTTON et al. 9 ] Adapted by permission from Macmillan Publishers Ltd: Nature, reference [9], copyright 1998.

is of special interest for research. The mechanism of the highly regulated SNARE-mediated membrane fusion of synaptic vesicles could offer possible approaches for development of suitable treatments. Further mechanistic insights might also be transferred to other membrane fusion events such as bacterial or viral infections. ${ }^{8}$

Since the late 1980s, SNARE proteins are intensively investigated regarding structure, mechanism and regulation. Nowadays, it is generally accepted that the SNARE proteins form the key components of membrane fusion along the secretory pathway. ${ }^{[-3}$ The neuronal membrane fusion of synaptic vesicles is mediated by the three SNARE proteins Synaptobrevin-2, also known as VAMP-2 (vesicle-associated membrane protein), Syntaxin-1A and SNAP-25 (25 kDa synaptosome-associated protein). The formation of the highly stable four helix bundle, the so-called SNARE complex, consisting of one helix each of Synaptobrevin-2 and Syntaxin-1A, and two helices of SNAP-25, provides the required energy for membrane fusion (figure 1.1). A variety of further executive and regulatory proteins have been identified such as Sec1/Munc18like (SM) proteins, CATCHR (complex associated with tethering containing helical rods) proteins, Complexins and Synaptotagmins, e.g. to maintain the dependency on $\mathrm{Ca}^{2+}$ influx of the neuronal membrane fusion. 3 [3.7]

After membrane fusion occured, the very stable SNARE complex is dissociated again into the free SNARE proteins due to energetical reasons and to close the cycle. The hexameric enzyme $N$-ethylmaleimide-sensitive factor (NSF) and its cofactors soluble NSF attachment proteins (SNAPs) catalyse the so-called disassembly reaction upon adenosine triphosphate (ATP) hydrolysis, and thereby free SNARE proteins are provided again. The enzyme itself exhibits no direct binding affinity for the SNARE complex. By binding of the cofactor $\alpha$-SNAP around the SNARE complex surface, NSF is able to catalyse the reaction. ${ }^{3+7.7}$ 
Besides recent progress in solving the structure of the disassembly machinery, the exact mechanism is still not completely understood. Especially, a specific inhibitor for NSF, which would facilitate crystallisation studies, is not identified until today. Hence, the first aim of this study is to synthesise a suitable inhibitor for the SNARE disassembly reaction which is based on the SNARE protein Synaptobrevin-2. Specific amino acid side chains of the Synaptobrevin-2 SNARE motif contributing in coiled coil formation, will be modified with sterically demanding side chains. On the surface of the SNARE complex, this sterical hindrance will prevent or weaken the binding of the cofactor $\alpha$-SNAP, and consequently, prevent SNARE disassembly reaction by NSF. The modified Synaptobrevin- 2 analogues are synthesised by solid-phase peptide synthesis (SPPS) and their properties regarding SNARE complex formation and disassembly are investigated by fluorescence anisotropy. This technique provides the advantage to monitor the individual steps of the disassembly reaction, such as $\alpha$-SNAP binding to the SNARE complex, and upon NSF addition, dissociation into the free SNARE proteins.

Furthermore, the second aim of this study is to investigate the Complexin binding to SNARE complexes containing the sterically modified Synaptobrevin-2 derivatives. Complexin is a small regulatory protein of the SNARE-mediated membrane fusion and binds to the groove between Synaptobrevin-2 and Syntaxin-1A. It is proposed to regulate the $\mathrm{Ca}^{2+}$ dependency of the neuronal membrane fusion together with the $\mathrm{Ca}^{2+}$ sensor Synaptotagmin. Influence on Complexin binding provides also influence on the regulation of SNARE complex formation. Therefore, the Complexin binding to the modified SNARE complexes is also monitored by fluorescence anisotropy.

In a third part of this study, the SNARE complex formation itself, the so-called zippering process, is addressed. It is believed that the coiled coil formation starts from the $N$-terminal parts of the SNARE motifs and proceeds like a zipper to the $C$-terminal part and the transmembrane domains. 10.12] Some executive or regulatory proteins are discussed to arrest the SNARE complex during this process to obtain partially zippered intermediates. 13 Especially, Synaptobrevin-2 mutants were previously investigated to get further insights into the zippering process. 11 12, 14 -19] The third aim of this study is to develop a Synaptobrevin-2 derivative which is arrested in the middle of the zippering process, but can be activated again by an external trigger, and the zippering reaction can proceed. For this purpose, photocleavable protecting groups (PPGs) are attached at the amino acid side chains contributing in the zippering process. By irradiation, the PPGs are specifically cleaved and an immediate "jump" in concentration of the active species can be observed. The modified amino acid building blocks are introduced by SPPS and the so-called caged Synaptobrevin-2 is investigated regarding the photocleavage properties of the PPGs and the SNARE complex formation properties before and after irradiation. 
In the last part of this study, Syntaxin transmembrane domains (TMDs) are synthesised by SPPS and labelled with different fluorophores at the $N$-terminal end of the sequences. The labelled TMDs are provided for investigations of Syntaxin clustering within model membrane systems with fluorescence-based methods, such as fluorescence resonance energy transfer (FRET). 


\section{SNARE-Mediated Membrane Fusion}

The evolutionary formation of membrane-enclosed organelles in eukaryotic cells gave the advantage of reaction separation and reaction localisation on membrane surfaces due to the impermeability of the lipid bilayer towards most hydrophilic molecules. For maintenance of communication between organelles and also between cells, the membrane barrier needed to be overcome by certain mechanisms. Besides transporter proteins and channels, transport vesicles filled with impermeable molecules represent one possibility of transport through the lipid bilayer. The vesicles are filled by the mechanism of endocytosis or membrane fission in which the corresponding membrane everts, surrounding the cargo molecules, and is subsequently clamped from the source membrane. On the other hand, the cargo molecules need to be released at the target compartment by the mechanism of membrane fusion or exocytosis. A machinery of specialised proteins ensures that the membranes are pulled in close proximity and that water molecules are excluded to fuse the transport vesicle with the target membrane. These two processes represent the basic mechanisms for cell communication and nutrition by transport vesicles. 1 [ 2]

Several life essential processes depend on endo- and exocytosis, for example immune reactions, reproduction, and synaptic transmission. Many of these events exhibit their own set of specialised proteins for regulation and execution of membrane fission and fusion. One family of specialised proteins is represented by the SNARE proteins, which mediate membrane fusion of transport vesicles along the secretory pathway of cells. By anchorage in the opposing membranes and formation of a highly stable coiled coil, SNARE proteins pull the membranes in close proximity and provide a certain amount of energy required for fusion. [3 [4] First identified as targets of clostridial botulinum and tetanus neurotoxins, ${ }^{3}$ [4] SNARE proteins were especially found to mediate neurotransmitter release at presynaptic nerve terminals. ${ }^{3 \cdot-7}$ Neurotransmitters are stored in synaptic vesicles at the presynaptic nerve terminal and are released into the synaptic cleft to maintain the communication between nerve cells. This so-called synaptic transmission is required for transferring of stimuli from one neuron via the synapse to the next neuron. 1 1 [2]

Dysfunctions of this process cause neuronal and/or neurodegenerative diseases or, like in the case of neurotoxins, paralysis which can lead to death of the organism. [4] 6] The highly regulated SNARE-mediated membrane fusion of synaptic vesicles is therefore of special interest for research as a possible approach for the development of suitable treatments. In addition, 
mechanistic insights into neuronal exocytosis might be transferred to other membrane fusion events such as bacterial or viral infections. []

\subsection{Synaptic Transmission}

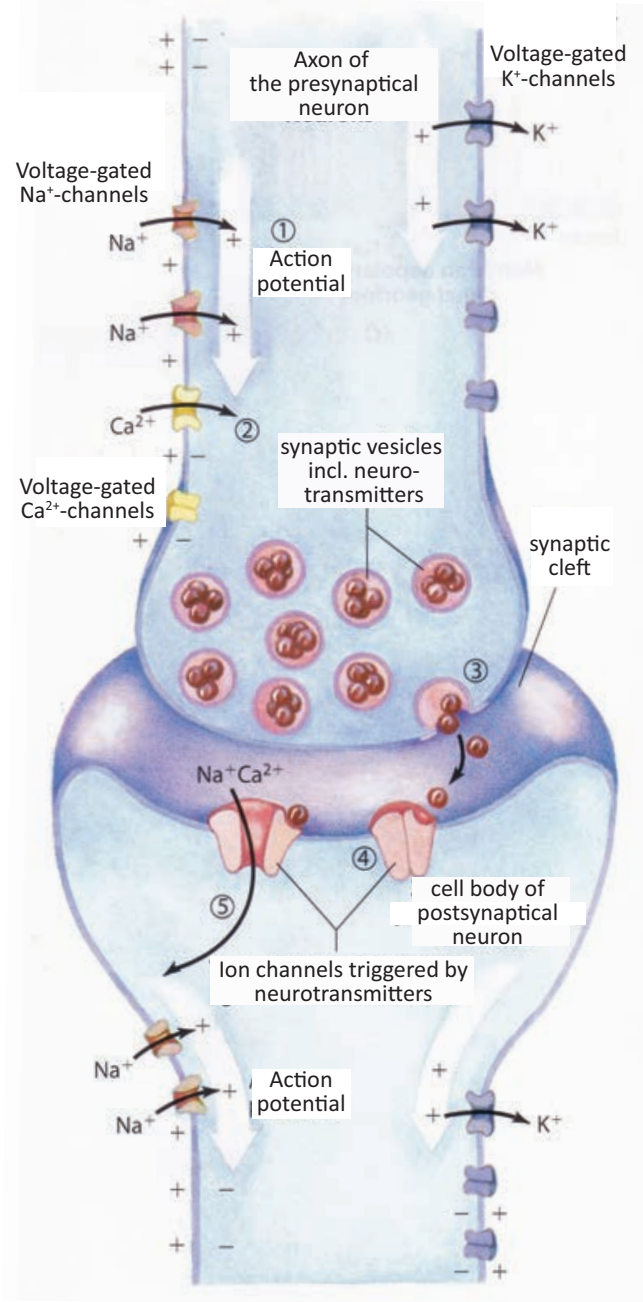

Figure 2.1: Scheme of synaptic transmission from the presynaptic neuron (top) via the synaptic cleft (middle) to the postsynaptic neuron (bottom). (1) Upon stimulation of the presynaptic neuron, an action potential propagates along the axon (white arrow), generated by voltage-gated $\mathrm{Na}^{+}$channels. The opening of these channels induces $\mathrm{Na}^{+}$influx, and thereby a local depolarisation of the plasma membrane. Propagation is achieved by triggering the opening of further $\mathrm{Na}^{+}$channels in close proximity. (2) Arrival of depolarisation at the nerve terminal triggers opening of $\mathrm{Ca}^{2+}$ channels and induces $\mathrm{Ca}^{2+}$ influx. (3) Increasing $\mathrm{Ca}^{2+}$ concentration triggers neuronal exocytosis of synaptic vesicles and thereby neurotransmitter release. The membrane fusion between the synaptic vesicle and the plasma membrane is mediated by the SNARE proteins Synaptobrevin-2, Syntaxin-1A and SNAP-25 in collaboration with further executive and regulatory proteins, like Synaptotagmin as $\mathrm{Ca}^{2+}$ sensor. (4) Neurotransmitters are released from the presynaptic neuron into the synaptic cleft and diffuse to receptors at the postsynaptic neuron. (5) Binding of neurotransmitters to receptors triggers opening of ion channels and $\mathrm{Na}^{+}$and $\mathrm{Ca}^{2+}$ influx induces depolarisation and thereby stimulation of the postsynaptic neuron. By this mechanism of synaptic transmission, the electrical signal of the presynaptic neuron is transformed to a chemical signal, thereby passed over the synaptic cleft, and afterwards in the postsynaptic neuron again transformed to an electrical signal. [2]

The importance of neuronal exocytosis can be illustrated by a closer look on its overall mechanism. The synaptic transmission is necessary for the transfer of a stimulus from one nerve cell via the synaptic cleft to the next nerve cell (figure 2.1). During this process, the electrical signal, called action potential, is transformed into a chemical signal within the synaptic cleft. Upon arrival of an action potential at the nerve terminal (figure 2.1 (1), opening of voltage-gated $\mathrm{Ca}^{2+}$ channels and thereby $\mathrm{Ca}^{2+}$ influx is induced (figure 2.1 (2)). The increase in $\mathrm{Ca}^{2+}$ concentration triggers the exocytosis of neurotransmitters from the synaptic vesicles into the synaptic cleft (figure 2.1 (3)). After diffusion through the synaptic cleft, the neurotransmitters bind to receptors at the plasma membrane of the postsynaptic nerve cell (figure 2.1 (4)) and thereby trigger the opening of ion channels. Influx of $\mathrm{Na}^{+}$and $\mathrm{Ca}^{2+}$ ions into the postsynaptic nerve cell induces depolarisation of the plasma membrane, and consequently stimulation of an action 
potential (figure 2.1 (5)). In this way, the electrical signal is transferred to the postsynaptic nerve cell and eventually forwarded to the next synapse. 112]

Synaptic transmission is a crucial process as impairments always have critical effects on the affected organism. For instance, this process is dysfunctional in neurodegenerative diseases or can be impaired by toxins, such as clostridial botulinum and tetanus toxins. These neurotoxins cause paralysis and can cause death by apnoea without treatment. The research of the exact mechanism of neuronal exocytosis can provide possible approaches for treatment of diseases or intoxications.

The process of neuronal exocytosis describes the membrane fusion between the synaptic vesicle and the plasma membrane. Interestingly, the influx of $\mathrm{Ca}^{2+}$ ions triggers membrane fusion of synaptic vesicles within milliseconds. This very fast response is challenging and requires a machinery of specialised proteins. The key elements are found to be the SNARE proteins Synaptobrevin-2, Syntaxin-1A and SNAP-25 which mediate the neuronal membrane fusion in collaboration with further executive and regulatory proteins. For example, Synaptotagmin-1 functions as a $\mathrm{Ca}^{2+}$ sensor. ${ }^{3-7}$ In the following chapters, structural and functional aspects of SNARE proteins as well as the mechanism of SNARE-mediated membrane fusion is discussed.

\subsection{SNARE Proteins and SNARE Complex}

SNARE (soluble $\underline{N}$-ethylmaleimide-sensitive factor attachment protein receptor) proteins were first identified around 1990. Especially, studies with clostridial botulinum and tetanus toxins revealed them as substrates for these highly specific proteases for inhibiting neurotransmitter release, and thereby their importance in neuronal membrane fusion was shown. ${ }^{3}$ 4 20-22] The name is based on the interaction with the previously identified components of membrane fusion NSF and SNAPs. 23. Today, it is generally accepted that the SNARE proteins form the key components of membrane fusion along the secretory pathway. ${ }^{3}$

Proteins of the SNARE family comprise a conserved domain of $60-70$ residues containing heptad repeats which is referred to as the characteristic SNARE motif. ${ }^{3+5}$, 7 In most of the SNARE sequences, these motifs are $C$-terminally connected to a transmembrane domain (TMD) by a small linker region. In addition, different $N$-terminal domains are among others the origin of further subgroups of the SNARE protein family. [3] [5]

The neuronal membrane fusion comprises a special case, due to its tight dependency on $\mathrm{Ca}^{2+}$ influx. ${ }^{[5]}$ The SNARE proteins Synaptobrevin-2, also known as VAMP-2 (vesicle-associated membrane protein), Syntaxin-1A and SNAP-25 (25 kDa synaptosomal-associated protein) were identified to mediate neurotransmitter release. Synaptobrevin-2 and Syntaxin-1A exhibit one 
SNARE motif each and a C-terminal TMD, whereas SNAP-25, as well as its homologues, represents an exception with two SNARE motifs joined by a palmitoylated linker for membraneattachment, instead of a TMD (figure 2.2 a). Among these three peptides, Synaptobrevin-2 constitutes the simplest domain structure with a short, unstructured and proline-rich $N$-terminal domain besides the SNARE motif and the TMD. In contrast, Syntaxin-1A exhibits a $N$-terminal domain that forms an antiparallel three-helix bundle and is referred to as Habc domain. In addition, at the very $\mathrm{N}$-terminal end, a short sequence called the $\mathrm{N}$-peptide is noteworthy. $\left.{ }^{3+5}, 7\right]$ It can be derived from the structure of Syntaxin-1A that it has more functions in membrane fusion than the other SNARE proteins. ${ }^{4]}$

a

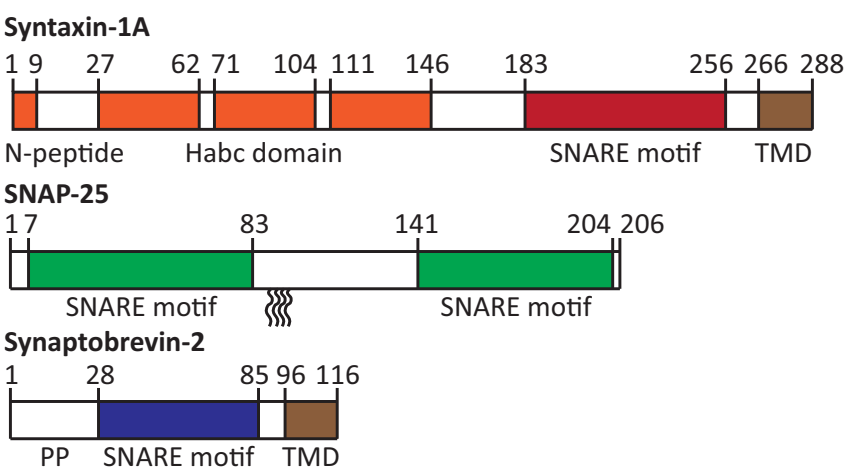

b

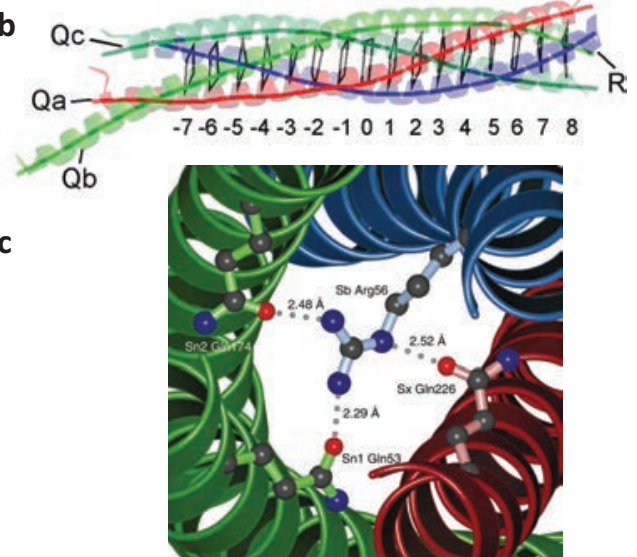

Figure 2.2: (a) Domain structure of neuronal SNARE proteins Syntaxin-1A, SNAP-25 and Synaptobrevin-2 including SNARE motifs, transmembrane domain (TMD), as well as Habc domain and $\mathrm{N}$-peptide of Syntaxin-1A, and proline-rich $N$-terminal part (PP) of Synaptobrevin-2. Figure modified from STEIN et al.[24] Adapted by permission from Macmillan Publishers Ltd: Nature, reference [24], copyright (C) 2009. (b) Crystal structure of neuronal SNARE complex including 16 layers $(-7-+8)$ as ribbon diagram ( $\mathrm{pdb}$ code $1 \mathrm{sfc}$ ). Complex consisting of SNARE motifs of Synaptobrevin-2 (R, blue), Syntaxin-1A (Qa, red), and SNAP-25 (Qb, Qc, green). Figure modified from KlOEPPER et al.25] Republished with permission of American Society for Cell Biology, from reference [25], copyright (C 2007; permission conveyed through Copyright Clearance Center, Inc. (c) Detailed structure of central hydrophilic zero layer of neuronal SNARE complex including Synaptobrevin-2 residue R56, Syntaxin-1A residue Q226, and SNAP-25 residues Q53 and Q174. Figure adapted from SuTton et al. 9 . Adapted by permission from Macmillan Publishers Ltd: Nature, reference [9], copyright (c) 1998.

Synaptobrevin-2 is predominantly located in synaptic vesicles, whereas Syntaxin-1A and SNAP25 are located in the plasma membrane at the nerve terminals, which led to their original classification into vesicle-SNAREs (v-SNAREs) and target-SNAREs (t-SNAREs). 3 6] As soon as their four SNARE motifs get in contact, a tight and highly stable coiled-coil of four parallel $\alpha$-helices is formed which is suggested to pull the opposing membranes into close proximity as well as to contribute the required energy for mediating membrane fusion. $3+5]$ The so-called neuronal SNARE complex was shown to be stable even against adverse conditions like high temperatures $\left(80{ }^{\circ} \mathrm{C}\right)$ or denaturing agents ( $8 \mathrm{M}$ urea or $\left.2 \% \mathrm{SDS}\right)$. 26] 27. The 
crystal structure revealed mainly hydrophobic interactions which are subdivided into 16 distinct layers (figure $2.2 \mathrm{~b}$ ). The only exception constitutes a central hydrophilic layer (zero layer) which was found to be highly conserved in all SNARE motif sequences and always consists of three glutamin $(Q)$ residues and one arginine $(R)$ residue (figure 2.2 c).9. On this basis, SNARE proteins were reclassified into Qa-, Qb-, Qc-, and R-SNAREs. [3-5] 7, 28]

Besides the formation of the ternary SNARE complex, which mediates membrane fusion, in vitro SNARE motifs tend to form a variety of homo- and hetero-oligomeres. Especially, the formation of the so-called binary complex consisting of SNAP-25's two SNARE motifs and two Syntaxin-1A molecules represents a dead-end along the reaction pathway. In this case, the binding site for Synaptobrevin is blocked and the second Syntaxin-1A cannot be replaced by Synaptobrevin. 111 29] In addition, the Habc domain of Syntaxin-1A exhibits an open or closed conformation (figure $2.3 \mathrm{a}$ and $\mathrm{b}$ ). In the closed conformation, the three-helix bundle of the Habc domain is associated to the SNARE motif (or H3 domain), and thereby suggested to prevent SNARE complex assembly. ${ }^{30] 31}$ Additionally, the Habc domain as well as the N-peptide of Syntaxin-1A were found to bind to the Sec1/Munc18-like (SM) protein Munc18-1 (figure 2.3 c). SM proteins are under discussion to be part of the SNARE assembly process during membrane fusion 31,32 and is addressed in more detail in chapter 2.5 , 3.-7]
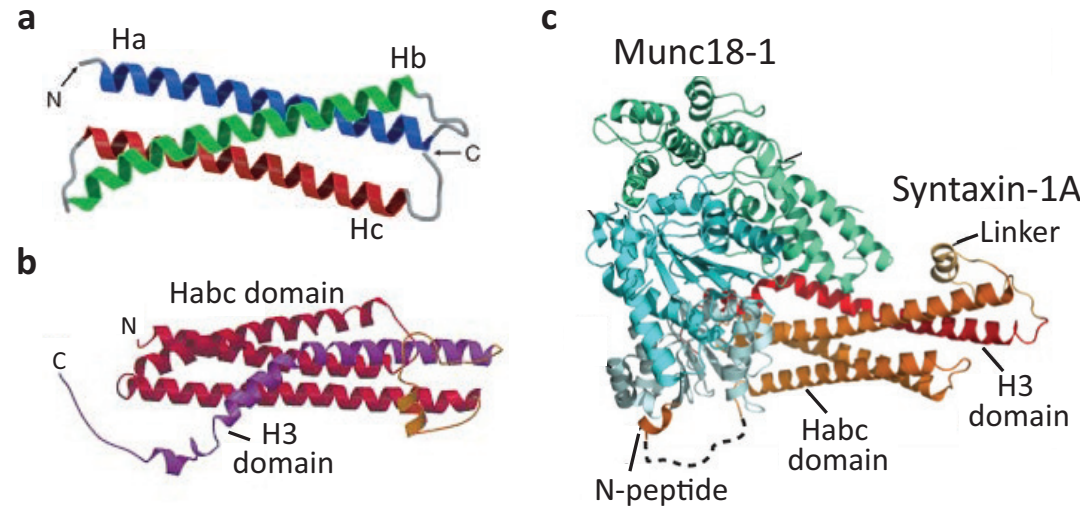

Figure 2.3: (a) Crystal structure of the open conformation of the $\mathrm{N}$-terminal Habc domain of Syntaxin-1A (pdb code 1br0) with the three $\alpha$-helices $\mathrm{Ha}$ (blue), $\mathrm{Hb}$ (green), and $\mathrm{Hc}$ (red). Figure modified from LERMAN et al. [30] Adapted with permission from reference [30]. Copyright (c) 2000 American Chemical Society. (b) Structure of closed conformation in which the Habc domain (red) is associated to the SNARE motif (H3 domain, purple). Figure modified from Misura et al. 31] Adapted by permission from Macmillan Publishers Ltd: Nature, reference [31], copyright (C) 2000. (c) Crystal structure of Munc18-1-Syntaxin-1A complex (pdb code 3c98) consisting of Munc18-1 (blue-green) and Syntaxin-1A (red-orange) in the closed conformation. Dashed lines represent flexible linker regions. Figure modified from JAhN \& FAsshauer. [5] Adapted by permission from Macmillan Publishers Ltd: Nature, reference [5], copyright (C) 2012.

These conformational as well as dead-end intermediates were among others considered to be responsible for the very slow in vitro SNARE complex assembly which usually takes several 
hours. ${ }^{[5}$ To overcome this problem, stabilised acceptor complexes ( $\Delta \mathrm{N}$ complex) are used in vitro consisting of SNAP-25, Syntaxin-1A and a C-terminal Synaptobrevin-2 fragment to obtain an available $N$-terminal binding site for full-length Synaptobrevin-2. Indeed, distinctly faster fragment displacement, and therefore, ternary SNARE complex formation was observed. $5[11]$

\subsection{A Closer Look at SNARE Protein Synaptobrevin-2}

The neuronal SNARE protein Synaptobrevin-2 or VAMP-2 is a small ( $\sim 12 \mathrm{kDa})$ synaptic vesicle protein. In 1988, it was firstly identified and isolated by TRIMBLE et al. from synaptic vesicles of the electromotor system of Torpedo californica. ${ }^{33}$ In 1989, Synaptobrevin-2 was named by BAUMERT et al. after the greek term synapto $(\sigma \psi \nu \alpha \pi \tau o)=$ to connect and the latin term brevis $=$ short. ${ }^{34}$ Only a few years later, in 1992, it was identified as an essential protein for membrane fusion because of specific cleavage by neurotoxins. 3 4 20] Initially classified as v-SNARE by Rothman et al. ${ }^{35]}$ due to main isolation from synaptic vesicles, Synaptobrevin-2 and the whole VAMP family was reclassified as R-SNAREs based on the highly conserved arginine residue within the zero layer. 28$]$

Among the neuronal SNARE proteins, Synaptobrevin-2 comprises the simplest domain structure with its short, unstructured, and proline-rich $N$-terminal domain $(1-27)$, its SNARE motif $(28-84)$, its short linker region $(85-95)$, and its $C$-terminal TMD $(96-116)$ (figure 2.2 a). 24. Together with its neuronal counterparts Syntaxin-1A and SNAP-25, it forms the highly stable neuronal SNARE complex which mediates membrane fusion. [3-5, 24]

The soluble part of Synaptobrevin-2 $(1-95)$ is mainly unstructured in aqueous solution, whereas the TMD $(96-116)$ forms an $\alpha$-helical structure. During assembly of the SNARE complex, extraordinary structural changes occur until helical conformation is reached. Studies using nuclear magnetic resonance (NMR) spectroscopy showed that Synaptobrevin-2 exhibits three helical regions in micelles of the detergent dodecylphosphocholine (DPC). [36] Two of these three helices comprises the SNARE motif region, separated by an unstructured region. The third helix consists of the TMD residues. Helix I $(36-54)$ and helix II $(77-88)$ are more flexible than helix III (93 - 115) (figure $2.4 \mathrm{~b}$ ). This structural arrangement was suggested to influence the assembly of the SNARE complex, as the $N$-terminal helix I could function as a nucleation site for complex formation. This would accelerate the $N$ - to $C$-terminal propagation of the coiled-coil by a preformed helical conformation. In addition, hydrophobic moment and hydropathy of the Synaptobrevin-2 sequence were analysed (figure $2.4 \mathrm{a}$ ). As expected, the TMD exhibits highest hydrophobicity within the sequence, and the SNARE motif shows a highly amphipathic region, especially around residue 44 . When modelled to a helical structure, the latter exhibits hydrophobic residues on one face and more hydrophilic regions on the other, which is consistent with hydrophobic residues contributing to SNARE complex formation and charged residues on the complex surface. Interestingly, a highly hydrophilic region was found 
which includes not only the zero layer but also a region comprising residues $\sim 56-65$ with the highest hydrophilicity at residues $58-61$. Within the structure in DPC micelles, this region is unstructured. [36] This is consistent with the identified SNARE complex binding sites for $\alpha$-SNAP (chapter 4.1) and Complexin (chapter 2.7.1) which are based on electrostatic interactions.
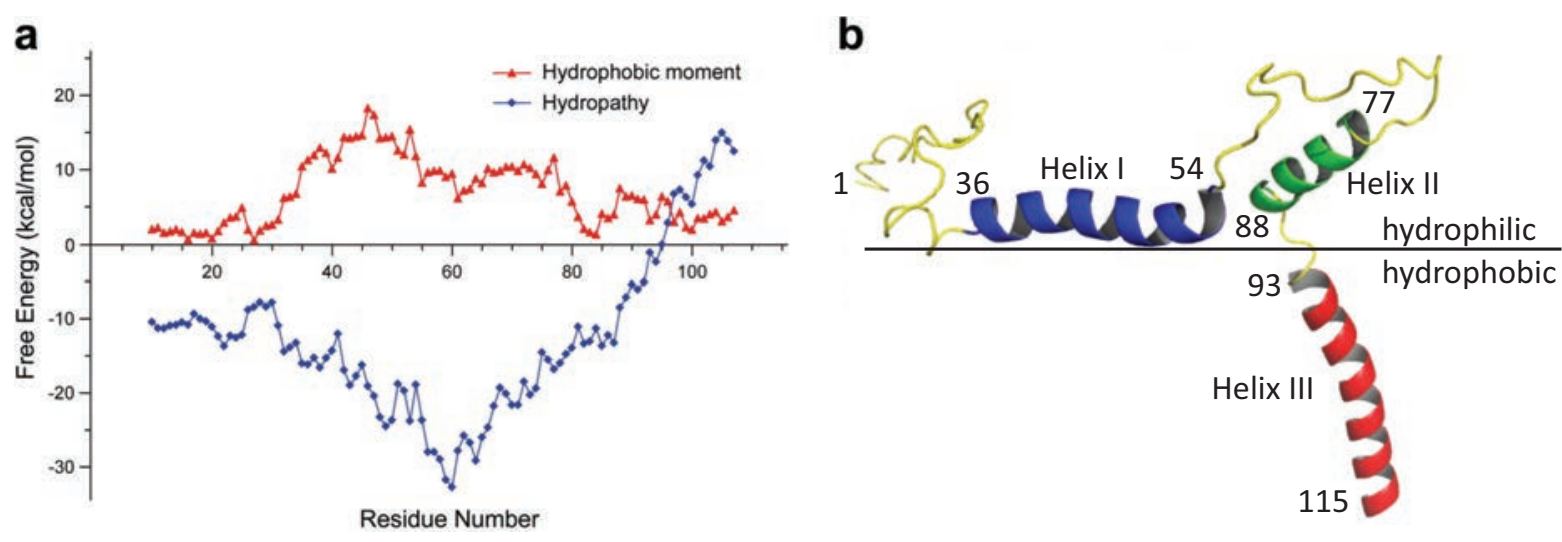

Figure 2.4: Structure of Synaptobrevin-2 $(1-116)$ in DPC micelles. (a) Analysis of hydrophobic moment and hydropathy with octanol-scale used for calculation of the free energy for transfer from bilayer to water. (b) Structure determined by NMR spectroscopy (pdb code $2 \mathrm{kog}$ ) with helix I (blue) at micelle surface, helix II (green) on hydrophilic site, and helix III (red) corresponding to the TMD on the hydrophobic site. Figure modified from ELLENA et al. 36]

More recently, these NMR studies were extended to bicelles and bilayers as well as to the usage of electron paramagnetic resonance (EPR) spectroscopy. For this purpose, Synaptobrevin-2 was reconstituted into DMPC/DHPC bicelles and POPC/POPS bilayers, respectively. It was found that the amount of helical conformation and association to the lipid interface decreases from micelles via bicelles to bilayers. This indicated that the structural conformation and interaction with the lipid head groups are strongly dependent on membrane curvature and were suggested to regulate Synaptobrevin-2's contribution to SNARE complex assembly. [37]

In 2010, a possible connection between Synaptobrevin-2 and certain neurodegenerative diseases was revealed by the discovery of $\alpha$-Synuclein binding to the $N$-terminal domain of Synaptobrevin-2. [38] $\alpha$-Synuclein is a small soluble protein which is enriched in presynaptic terminals. At the $N$-terminal end it exhibits an amphipathic helix for membrane interaction and with its $C$-terminal negatively charged domain it was found to bind to Synaptobrevin-2. $38+40$ $\alpha$-Synuclein was discovered to be the main component of fibrils in the so-called Lewy bodies which were identified to play a key role in Parkinson's disease and dementia. It is therefore of special interest in the research field of neurodegenerative diseases. 66 38, 39, 41 The interaction with Synaptobrevin-2 suggested the physiological function of $\alpha$-Synuclein as a SNARE complex chaperone, and hence, loss of this function was thought to initiate neurodegeneration. This 
hypothesis is still debated because there is evidence that the pathological effect of $\alpha$-Synuclein by formation of neurotoxic aggregates is not correlated to a loss of physiological function. ${ }^{39]}$

In contrast, $\mathrm{CHOI}$ et al. recently showed that a cooperative effect between $\beta$-Amyloid and $\alpha$-Synuclein induces oligomerisation of larger $\alpha$-Synuclein aggregates. ${ }^{41]} \beta$-Amyloid was found to be the major component of plaques and aggregates associated with Alzheimer's disease, in contrast to Parkinson's disease and dementia. But also combinations were found like $\beta$-Amyloid plaques in dementia patients or Lewy bodies containing $\alpha$-Synuclein in Alzheimer's patients, respectively, which often shows more severe progress of the diseases. Indeed, larger $\alpha$-Synuclein aggregates obtained by seeding with $\beta$-Amyloid were shown to inhibit SNARE-mediated vesicle fusion in vitro and in PC12 cells. On the other hand, aggregates formed only by $\beta$-Amyloid or $\alpha$-Synuclein, respectively, did not inhibit vesicle fusion. In addition, the inhibitory effect of these aggregates was shown to be based on the interaction with the $N$-terminal domain of Synaptobrevin-2. Probably, the large $\alpha$-Synuclein/ $\beta$-Amyloid aggregates bind to Synaptobrevin2 , and thereby prevent interaction with Syntaxin-1A and SNAP-25 to form the SNARE complex. This was consistent with observed clustering of Synaptobrevin-2-containing vesicles. These results indicated that not only the aggregates formed by $\alpha$-Synuclein or $\beta$-Amyloid alone, which are markers for diagnosis of neurodegenerative diseases, have a dramatic effect on neuronal functions. But also the induced $\alpha$-Synuclein aggregation by $\beta$-Amyloid or other seeding factors, like dopamin, probably exhibit the highest neurotoxicity due to direct inhibition of SNARE-mediated neurotransmitter release. ${ }^{41}$

In addition, a variety of studies were performed using Synaptobrevin-2 mutants to investigate the SNARE zippering process. ${ }^{[11}[12$ [14-19] This issue is discussed in detail in chapter 5.1 .

\subsection{The Cycle of SNARE-Mediated Membrane Fusion}

SNARE-mediated membrane fusion proceeds in a cycle of complex formation (SNARE assembly, chapter 2.5), which was proposed to induce fusion, and afterwards ATP-dependent dissociation of the SNARE complex (SNARE disassembly, chapter 2.6) catalysed by NSF and its cofactor $\alpha$-SNAP (figure 2.5). 3. In more detail, the cycle was suggested to begin from free SNARE clusters of Qa-, Qb-, and Qc-SNAREs in the acceptor membrane which, probably dependent on Sec1/Munc18-like (SM) proteins, form the acceptor complex. Upon approach of an R-SNAREcontaining vesicle, the loose trans-SNARE complex is formed by interaction of the $\mathrm{N}$-terminal parts of the corresponding SNARE motifs. In the trans-configuration, TMDs of contributing SNARE proteins are anchored in two opposing membranes. Complex assembly is suggested to proceed from $\mathrm{N}$ - to $\mathrm{C}$-terminal end of SNARE motifs to form the tight trans-SNARE complex, whereby regulatory proteins such as Complexins (chapter 2.7) and Synaptotagmins (chapter 2.7.3) are proposed to trigger the assembly process in response to $\mathrm{Ca}^{2+}$ influx in 


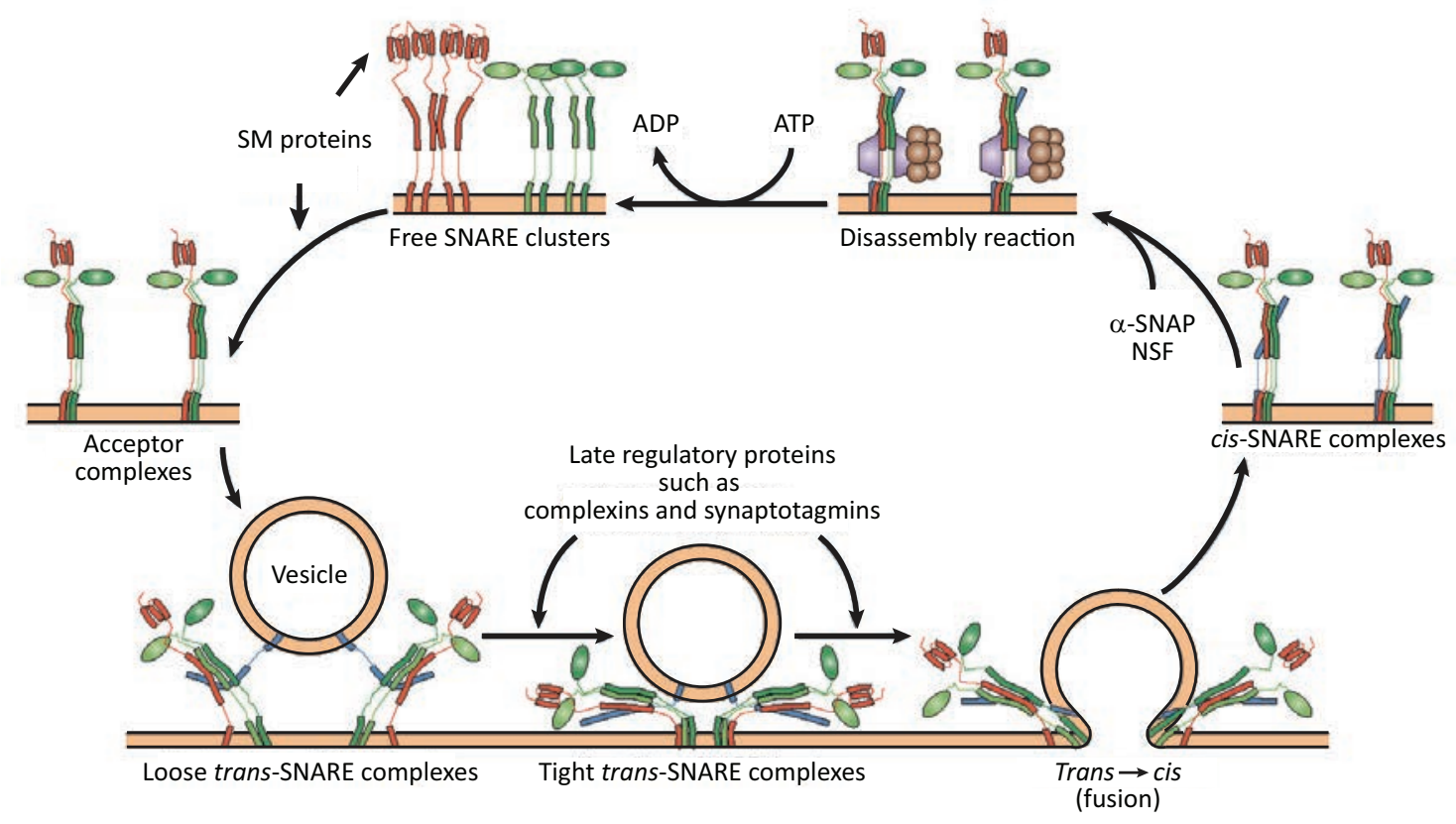

Figure 2.5: Cycle of SNARE-mediated membrane fusion including free SNARE clusters of Qa-SNAREs (red), Qb-, and Qc-SNAREs (green) in the acceptor membrane (top left), formed acceptor complexes (middle left), approaching R-SNARE (blue)-containing vesicle and formation of loose trans-SNARE complexes (bottom left), possible regulation of the tight trans-SNARE complex by regulatory proteins (Complexins/Synaptotagmins) (bottom middle), transformation from trans- to cis-complexes and membrane fusion (bottom right), cis-SNARE complexes within the acceptor membrane (middle right), association of NSF (brown balls) and $\alpha$-SNAP (purple trapezium) around the cis-complexes (top right), and finally, ATP-dependent Disassembly of cis-SNARE complexes into free SNARE proteins. Figure modified from JAHN \& SCHELLER. [3] Adapted by permission from Macmillan Publishers Ltd: Nature Reviews Molecular Cell Biology, reference [3], copyright (c) 2006.

neuronal exocytosis. The transformation from trans- to cis-SNARE complexes in combination with Synaptotagmin is hypothesised to open the fusion pore. Within the cis-complex, all contributing SNAREs are finally anchored in the same membrane, and this highly stable complex has to be dissociated again into the free SNARE proteins due to energetic reasons. The cofactor $\alpha$-SNAP bind around the SNARE complex and induce binding of hexameric NSF which catalyses, upon ATP-hydrolysis, the so-called disassembly reaction. Finally, free SNARE proteins are provided again and by various mechanisms sorted to their corresponding target localisation (e.g. Synaptobrevin-2 anchored in synaptic vesicles). ${ }^{[3-7]}$

\subsection{Priming and Assembly of the SNARE Complex}

The cycle of membrane fusion mediated by SNARE proteins was suggested to start from free SNARE clusters and afterwards formation of the SNARE acceptor complex comprising Syntaxin-1A and SNAP-25 (figure 2.5). 3. Recently, this starting point was questioned due to the increasing evidence that SM proteins, such as Munc18, and CATCHR (complex associated 
with tethering containing helical rods) proteins, like Munc13, also play key roles in membrane fusion. 4-7. It was even pointed out that the assembly of SNARE/SM complexes mediates fusion. [6] Physiologically, the combination of SNAREs, Munc18s, and Munc13s, as key components, is needed for neuronal exocytosis. This was already indicated by studies with Munc18-1 knockout mice ${ }^{42}$, and Munc13-1 and -2 double knockout mice ${ }^{43}$, respectively, which in both cases exhibited a total loss of neuronal exocytosis. $4-7.7$

As already mentioned in chapter 2.2, the SM protein Munc18-1 was found to bind to the Habc domain of Syntaxin-1A in the closed conformation ${ }^{31}$, to the N-peptide of Syntaxin-1A as well as to the ternary SNARE complex ${ }^{44}$ (figure 2.3). Initially, the formed Syntaxin-1AMunc18-1 complex was thought to inhibit SNARE assembly due to the blocked SNARE motif of Syntaxin-1A, but this would be totally contrary to the knockout studies. At this point, the interaction with the N-peptide of Syntaxin-1A seemed to be crucial because it comprises the connecting point between the interaction with the closed conformation and Syntaxin-1A in the open conformation assembled to the SNARE complex. For the transition from closed to open conformation, Munc13s were suggested to be important. $4-7$ ]

Structurally, Munc13s comprises two C2 domains for calcium binding and the so-called MUN domain which represents the key functional unit. 45.7 It could be shown that the isolated MUN domain of Munc13-1 interacts with SNARE complexes reconstituted in membranes, 45 with Syntaxin-1A/SNAP-25 heterodimers (Syntaxin-1A/SNAP-25, 1/1), 45 46] and also with low affinity with Munc18-1 and the Syntaxin-1A SNARE motif.47 In addition, it was shown that the MUN domain supports the conformational change of the closed Syntaxin-1A-Munc18-1 complex to the ternary SNARE complex, probably by opening the Syntaxin-1A conformation and thereby increasing the availability of Syntaxin-1A to contribute to SNARE assembly. 47] Taken together, these recent findings suggested a different starting point for the cycle of SNARE-mediated membrane fusion, namely the complex consisting of closed Syntaxin-1A and Munc18-1. Consequently, the assembly of the ternary SNARE complex is arranged by the cooperation of Munc13s and Munc18s, probably resulting in a partially zippered SNARE complex in the primed state. In addition, there is increasing evidence that one or both Muncs remain bound to the tight trans-SNARE complex due to the fact of counteracting premature disassembly by NSF/ $\alpha$-SNAP. 13] Also their crucial importance for neuronal exocytosis 42 , 43] as well as membrane-dependent affinity ${ }^{45}$ militates for their action also after assembly. 4 (5) 7] Nevertheless, essential questions regarding the starting point remain open. Consequently, no final definition can be set yet. ${ }^{7}$

The importance of SM and CATCHR proteins in vivo was underlined by the investigation of SNARE complex formation in vitro. A variety of $Q$ - and R-SNARE combinations were found, such as Qaabc (binary) complexes, 11 12] which in vivo needed to be prevented and channeled towards formation of QabcR-SNARE complexes. 
In addition to setting the starting point, Munc13s were also suggested to contribute in the localisation of the whole fusion machinery within the so-called active zone. This is the region where synaptic vesicles are localised in the nerve terminal in their docked and primed state, ready to release the neurotransmitters upon $\mathrm{Ca}^{2+}$ influx. The active zones of the plasma membrane contain specialised proteins to guide the synaptic vesicles during this preparing step prior to membrane fusion. Besides structural organisation of the machinery components, the close localisation to the $\mathrm{Ca}^{2+}$ channels for fast response upon $\mathrm{Ca}^{2+}$ triggering represents the second task of active zone proteins. Munc13s were suggested to constitute the connecting point between these two tasks by interaction with the SNARE/SM protein complexes on the one hand, and interaction with active zone proteins, such as RIM (Rab3-interacting molecule) and RIM-BP (RIM-binding proteins), on the other hand. [4-6] The formation of a complex consisting of Munc13, RIM and RIM-BP results in tethering of plasma membrane and synaptic vesicle, first by binding of RIM to the vesicular GTPases Rab3 and Rab27, and second by binding of RIM and RIM-BP to $\mathrm{Ca}^{2+}$ channels (figure 2.6). 4. 6]

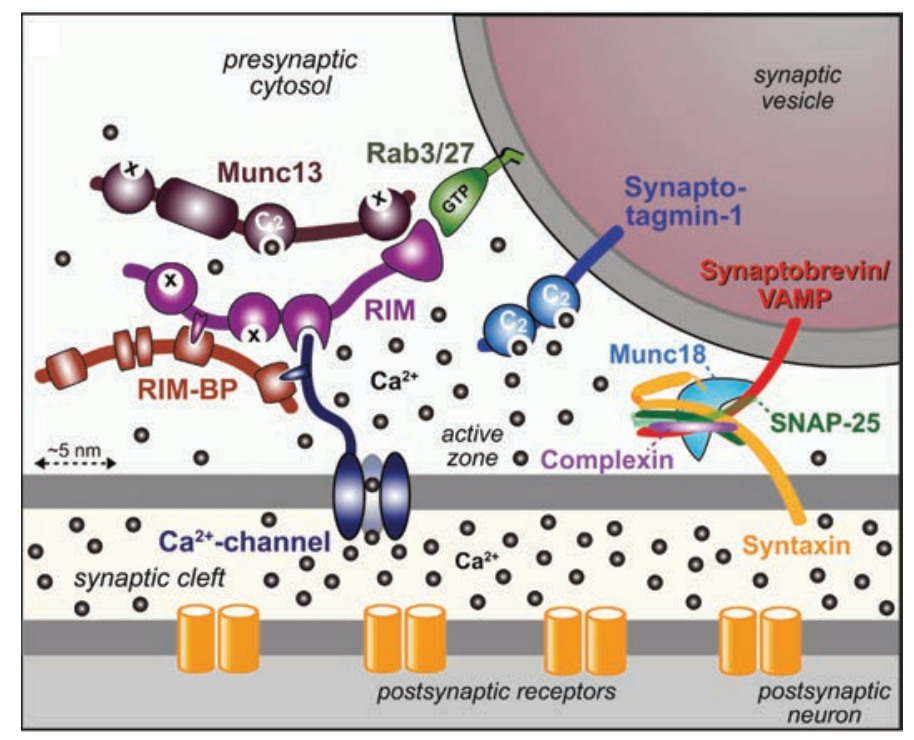

Figure 2.6: Schematic localisation of neuronal membrane fusion machinery at the active zone. Synaptic vesicle (top right) in the docked state including the key functional units for neurotransmitter release: (right) SNARE/SM protein complex consisting of Synaptobrevin-2 (red), Syntaxin-1A (yellow), SNAP-25 (green), and Munc18 (light blue). In addition, regulatory protein Complexin (light purple) bound to the SNARE complex. (middle) $\mathrm{Ca}^{2+}$ sensor protein Synaptotagmin-1 (blue), and (left) active zone protein complex containing Munc13 (dark brown), RIM (dark purple), RIM-BP (light brown) bound to vesicular GTPase Rab3/27 (light green) on the vesicle site, and to $\mathrm{Ca}^{2+}$ channel (dark blue) on the plasma membrane site. Figure used from SüDHOF. [6] Reprinted from reference [6], Copyright (c) 2013, with permission from Elsevier.

Localisation by active zone proteins as well as interaction with SM proteins arrange SNARE proteins into close proximity to assemble to the stable four-helix bundle upon contact. With a variety of studies it was confirmed that the SNARE complex formation begins from the $N$ terminal part of the SNARE motifs and proceeds towards the $C$-terminal part like a zipper. 10 -12] 
In this way, SNARE assembly pulls the opposing membranes together and brings them in close proximity for fusion. ${ }^{[3-5]}$ Especially, Synaptobrevin-2 mutants were investigated to get further insights into the zippering process. 11 [12 14-19]. These studies is discussed in more detail in chapter 5.1 .

After the synaptic vesicle and the whole participating fusion machinery is set to the docked and primed state, the system has to react to $\mathrm{Ca}^{2+}$ influx which is known to trigger neuronal exocytosis. This is found to be mediated by the $\mathrm{Ca}^{2+}$ sensor Synaptotagmin in possible combination with Complexins which is described in detail in chapter 2.7.3.

To conclude, the shown cycle of SNARE-mediated membrane fusion in figure 2.5 still represents an up-to-date possible mechanism but focuses only on the key components, the SNARE proteins. Especially for the SNARE assembly, the starting point, the Q-SNARE acceptor complex, is under discussion. Nevertheless, it gives a direct overview of the mechanism without causing confusion by including all participating proteins. However, to understand the whole process of neuronal exocytosis, it is necessary to investigate each protein as well as the overall interplay between them which is a challenging task with this amount of contributors. In the following chapter the SNARE disassembly reaction is discussed in detail.

\subsection{The NSF $/ \alpha$-SNAP-Mediated SNARE Disassembly Reaction}

After membrane fusion occurred, SNARE complexes are in cis-configuration, and all proteins of the complexes are located together in the fused membrane. In this configuration, the SNARE complexes exhibit a high stability, are inactive and have to be recycled for the next round of membrane fusion. To dissociate the cis-SNARE complexes, a remarkable amount of energy is required. The so-called disassembly reaction is catalysed by the enzyme $N$-ethylmaleimidesensitive factor (NSF) and its cofactors soluble NSF attachment proteins (SNAPs) upon ATP hydrolysis. $3-7$. The functional machinery, which is necessary for SNARE disassembly reaction, consists of NSF, SNAPs and the SNARE complex. It is called $20 \mathrm{~S}$ particle or $20 \mathrm{~S}$ (super)complex due to its sedimentation coefficient of 20 Svedberg units. 48, 49] The exact composition, especially the needed number of SNAPs, is still under discussion.

\subsubsection{N-ethylmaleimide-sensitive factor (NSF)}

In 1988, Rothman et al. firstly identified NSF to contribute in trafficking of eukaryotic cells. ${ }^{[50-52]}$ From these early studies, NSF derived its name due to the observed inhibition of protein transport by $\mathrm{N}$-ethylmaleimide, which could be restored by adding NSF-containing cell extract. 51 [52] 
NSF is a homohexameric type II ATPase with a molecular weight of $\sim 500 \mathrm{kDa}$ and belongs to the AAA+ (ATPases associated with various cellular activities) superfamily. ${ }^{3}[3]$ Meanwhile, it is one of the best-investigated members of this family. ${ }^{53}$. The AAA + ATPases all have in common that they transfer chemical energy from ATP hydrolysis into conformational changes, and subsequently induce mechanical action on their substrates. The functions of these enzymes are diversely distributed ranging from protein complex disassembly, like NSF, to e.g. DNA unwinding. 53 [54] Structurally, the main feature of this protein family is the ATP-binding domain (AAA domain) with about $200-250$ amino acid residues. ${ }^{54}$

In the case of NSF, one subunit consists of three domains: the $N$-terminal $N$ domain $(1-205)$, and two ATPase domains D1 (206 - 477) and D2 (478 - 744) (figure 2.7 and 2.8 a). The $\mathrm{N}$ domain functions as the binding site for the cofactors and substrate, the D1 domains exhibits most of the ATPase activity, and the D2 domain provides the hexamerisation of the protein. [53] [55] The crystal structures of the $\mathrm{N}$ domain ${ }^{[56]}$ and $\mathrm{D} 2$ domain ${ }^{57}$ were published in the late 1990s and showed two subdomains $\left(\mathrm{N}_{\mathrm{A}}\right.$ and $\left.\mathrm{N}_{\mathrm{B}}\right)$ for the $\mathrm{N}$ domain as well as for the D2 domain $(\alpha$ and $\alpha / \beta)$, with the latter to be generic for AAA+ domains (figure 2.7). .53 Nevertheless, crystal structures of the D1 domain or full-length NSF are still not available.

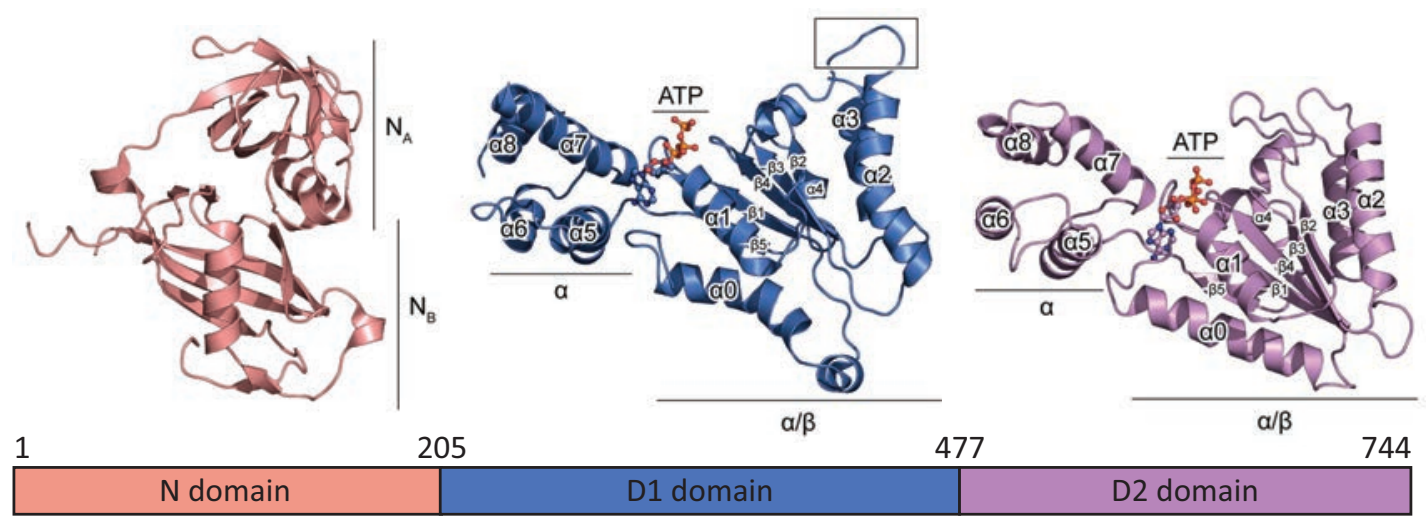

Figure 2.7: Domain structure of NSF with crystal structures of the $N$ domain ${ }^{[56}$ (pdb code $1 \mathrm{qcs}$ ) and the D2 domain [57] (pdb code 1nsf). Structure of the D1 domain $[50$ is derived by cryo-EM (pdb code 3j94). Figure modified from ZHAO et al. 53.

Continuous improvement of cryo-electron microscopy (EM) and NSF purification led to recently published near-atomic resolution structures of full-length NSF. 50 53] In 2015, BRUNGER et al. could solve the structures in ATP-bound and ADP-bound state of NSF with a resolution of $4.2 \AA$ and $7.6 \AA$, respectively (figure 2.8). .50$]$

From these structures, it was also possible to create a model of the D1 domain, which exhibits the characteristic $\alpha$ and $\alpha / \beta$ subdomains. In contrast to the D2 domain, it shows two helices twisted differently, one longer helix, as well as a pore loop with the typical sequence YVG (figure 2.7). 50 53] 
a

NSF

1

N domain

205

477

D2 domain 744

b

ATP-bound NSF

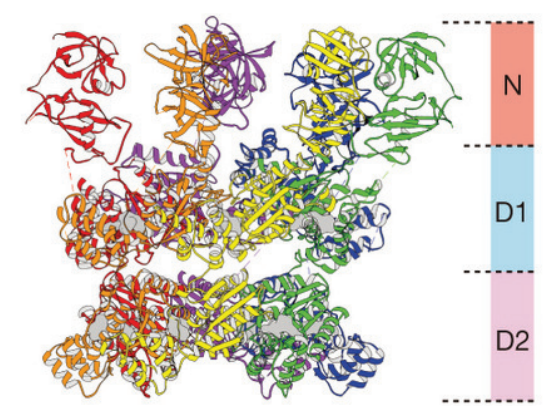

c

ADP-bound NSF

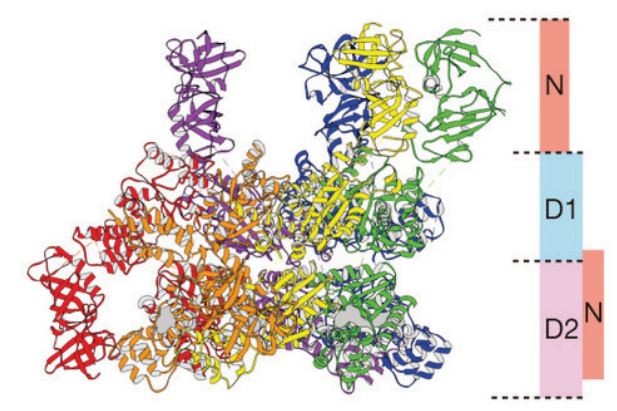

d

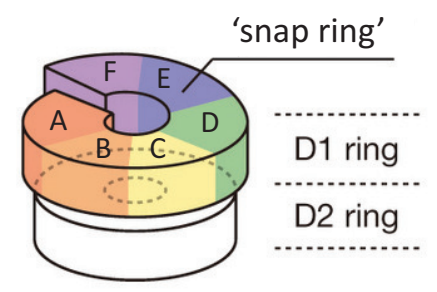

e

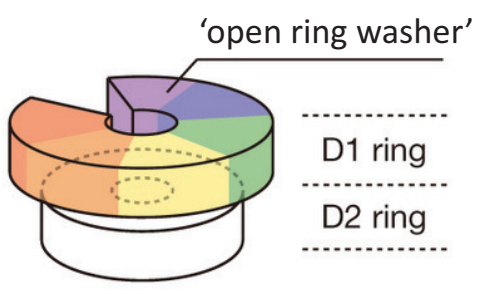

Figure 2.8: (a) Domains of NSF, ( $\mathbf{b}$ and $\mathbf{c}$ ) structures of full-length NSF in ATP-and ADP-bound state as side-views and ( $\mathbf{d}$ and $\mathbf{e}$ ) schematics to show topology of the D1 ring with colour-coded chains A to F. Figure modified from ZHAO et al. 50] Adapted by permission from Macmillan Publishers Ltd: Nature, reference [50], copyright (C) 2015.

In the recent structure of full-length NSF in the ATP-bound state, the six D2 domains are arranged as a 6-fold symmetric, planar ring and form the bottom of the structure. The six D1 domains are arranged like a snap ring on top of the D2 ring. The domains are stepping up anti-clockwise except for the distance between the subdomains $\mathrm{A}$ and $\mathrm{F}$. On top, the $\mathrm{N}$ domains are flexibly arranged and no symmetry was detected (figure 2.8 b and d). 50,53

In the ADP-bound state, the bottom D2 ring shows some slight differences, whereas the D1 ring is broader and exhibits an opening, like an open ring washer. Four $\mathrm{N}$ domains are still on top of the structure, whereas two domains are arranged down along the D1 and D2 domains (figure 2.8 c and e). 50 [53] The latter finding is consistent with previous cryo-EM structures from CHANG et al. where they proposed the existence of an up (ATP-bound) and down (ADP-bound) conformation of the $\mathrm{N}$ domains. ${ }^{55}$.

The comparison of both structures revealed the origin of the ATP-initiated conformational changes in the D1 domains, whereupon the $\mathrm{N}$ domains and D2 domains are restructured. All these structural movements have to be coupled to the disassembly of the SNARE complex. 50 [53]

With these higher-resolution structures, including the $20 \mathrm{~S}$ (super)complex, it was confirmed 
that full-length NSF, especially its N domains exhibits no binding site for the substrate (SNARE

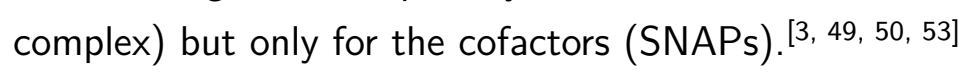

\subsubsection{Soluble $N$-Ethylmaleimide-Sensitive Factor Attachment Proteins (SNAPs)}

SNAPs are essential for the disassembly reaction due to no direct binding of NSF to the substrate, the SNARE complex. Without binding of SNAPs, the SNARE complex is not disassembled. On the other hand, NSF only binds to SNAPs, when the SNAP/SNARE subcomplex is already formed or to a trimerised mutant of SNAPs. [49] Three isoforms of SNAPs are identified: $\alpha-, \beta$ - and $\gamma$-SNAP. 30 [4] Only $\beta$-SNAP is an exception due to its expression limited to the brain, the other two isoforms are expressed in all cells of an organism. 49]

In the context of the SNARE disassembly reaction, $\alpha$-SNAP is the most-investigated isoform but still there is no crystal structure available. Only the crystal structure of its homologue from Saccharomyces cerevisiae (Baker's yeast) Sec17p provides indications for the interaction of $\alpha$-SNAP with NSF and the SNARE complex ${ }^{[58]}$ and is still used to refine recent structures. ${ }^{50}[55]$

Sec17p forms 14 antiparallely arranged $\alpha$-helices and exhibits two main domains: an $N$ terminal twisted sheet consisting of nine, anti parallel-arranged $\alpha$-helices, and a $C$-terminal $\alpha$-helical bundle. The $N$-terminal domain shows a right-handed twist, consequently, forming a concave and convex side of the domain. The $\mathrm{N}$ - and the $\mathrm{C}$-terminal ends of the protein are positioned at opposite parts of the structure (figure 2.9]. [58]
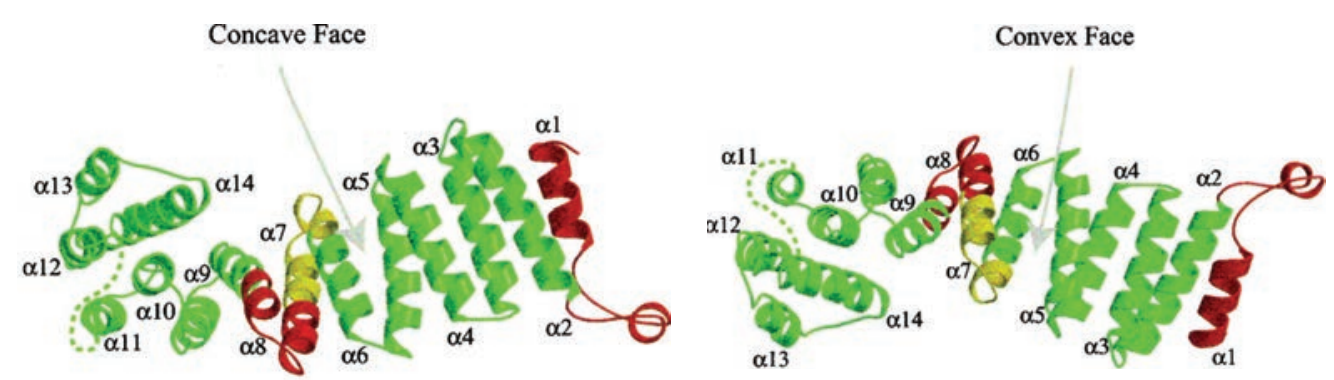

Figure 2.9: Crystal structure of Sec17 with the $14 \alpha$-helices showing the concave (left) and

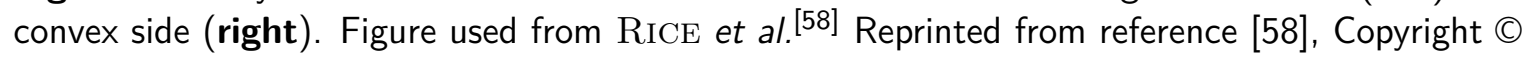
1999, with permission from Elsevier.

In the $N$-terminal domain, a longer loop connects the first two helices of the twisted sheet and the loop is arranged further away from the other helices. It exhibits mainly hydrophobic amino acids $(27-32)$ and is found to be the membrane-attachment site of $\alpha$-SNAP. WINTER et al. could show that $\alpha$-SNAP binding to the SNARE complex, as well as its efficiency during disassembly reaction, is increased when the SNARE complex is reconstituted into liposomes. 
They developed two $\alpha$-SNAP mutants, one lacking the entire $N$-terminal membrane anchor region $(33-295)$, and the second with two conserved phenylalanines mutated to serines (F27S, F28S). Both mutants indicated lower disassembly efficiency on liposomes as compared to the wild-type, and the second mutant was able to promote disassembly in solution at higher concentrations. ${ }^{59]}$ More recently published, PARK et al. were able to confirm that the binding efficiency of the $\alpha$-SNAP (F27S, F28S) mutant to the SNARE complex is comparable to the wild-type, on lipid-bound complex as well as in solution, but that the disassembly reaction is slower. On the other hand, the $\alpha$-SNAP $(33-295)$ mutant does not bind to the SNARE complex at all and no disassembly reaction occurs. 60 These results underline the influence of the membrane-attachment site of $\alpha$-SNAP. Consequently, it is necessary for investigations of the disassembly reaction to perform all experiments on lipid-bound SNARE complexes.

Recently, it was also shown by PARK et al.60] that $\alpha$-SNAPs exhibit an inhibitory effect on SNARE complex zippering. This issue is discussed in chapter 2.7.4.

$\alpha$-SNAP is needed for NSF binding, but little was known neither about the distinct interacting positions (NSF/ $\alpha$-SNAP and $\alpha$-SNAP/SNARE complex) nor about $\alpha$-SNAP stoichiometry within the $20 \mathrm{~S}$ complex. However, the question question remains, how the conformational change of NSF results in SNARE complex disassembly.

\subsubsection{Structure of the $20 \mathrm{~S}$ (super)complex}

Since 1997 it has been attempted to solve the structure of the $20 \mathrm{~S}$ (super)complex, first by quick-freeze/deep-etch EM ${ }^{[61]}$, by negative-staining EM ${ }^{[62]}$, and more recently by cryo-EM ${ }^{[55]}$. Due to low resolution $(>11 \AA)$ and sample preparation, these studies did not show neither clear structures for the D1 domain of the full-length NSF, nor for SNAPs and the SNARE complex in the $20 \mathrm{~S}$ (super)complex (figure 2.10). 53.

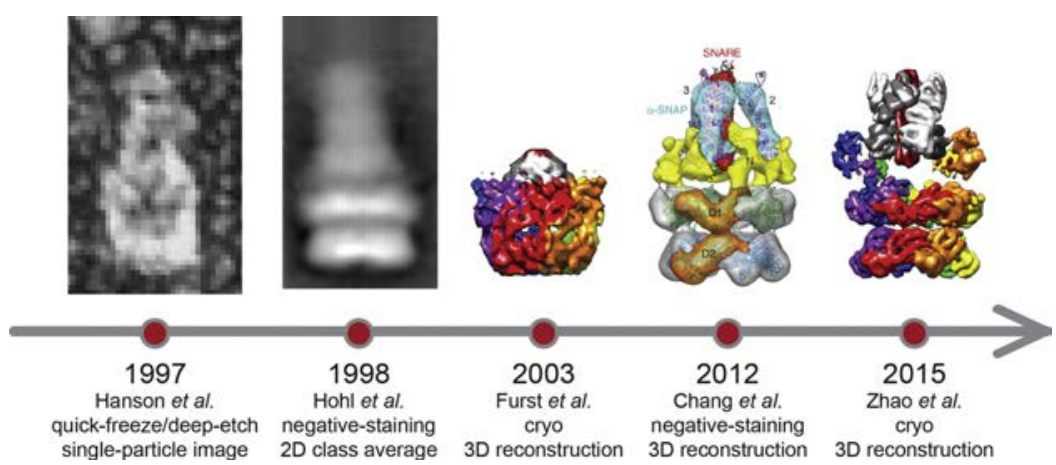

Figure 2.10: Timeline of structural studies on the $20 \mathrm{~S}$ (super)complex with representative images or reconstructions from quick-freeze/deep-etch single particle imaging by HANSON et al.61], negative-staining EM by HoHL et al. ${ }^{[62]}$, and cryo-EM by FURST et al. ${ }^{[63]}$, CHANG et al. [55], and ZHAO et al. [50]. Figure adapted from ZHAO et al. [53] 
With the recently published cryo-EM structures of full-length NSF, it was also possible to produce higher-resolution structures of the $20 \mathrm{~S}$ complex at a resolution of $7.6 \AA$ to $8.4 \AA$. The adenosine $5^{\prime}$-( $\beta, \gamma$-imido)triphosphate (AMPPNP)-bound $20 \mathrm{~S}$ complex is organised like a tower with the D2 and D1 rings of NSF hexamer at the basement, and $\alpha$-SNAPs and the SNARE complex at the spire encircled by the N domains of NSF. Within the structure, hardly any symmetry was found: at the basement a roughly six-fold symmetry is identified but at the top a pseudo four-fold symmetry is present. 50,53

In the case of the $20 \mathrm{~S}$ complex including the neuronal SNARE complex, BRUNGER et al. were able to observe four $\alpha$-SNAP molecules surrounding the SNARE complex. The distinct interactions between NSF $\mathrm{N}$ domains and $\alpha$-SNAPs were identified by mutagenesis studies. Based on the structure of the $20 \mathrm{~S}$ complex, $\alpha$-SNAP mutants were expressed and analysed regarding their disassembly efficiency. Two binding sites were detected where mutation affected the disassembly reaction. Both are located at the $C$-terminal end of $\alpha$-SNAP and are negatively charged: binding site I (D217, E249, E252, and E253) and binding site II (D290, E291, E292, and D293). 50] [53] On the other hand, one $\mathrm{N}$ domain exhibits one binding site with positively charged residues (R10, R67, K68, and K104) (figure 2.11 a).

This leads to a mismatch of binding modi: Four $\alpha$-SNAPs with an overall of eight binding sites facing six $\mathrm{N}$ domain binding sites. Consequently, each $\alpha$-SNAP interacts with at least one $\mathrm{N}$ domain. This results in two configurations for the six $\mathrm{N}$ domains surrounding four $\alpha$-SNAPs: Each $\mathrm{N}$ domain pair is arranged as direct neighbours or on opposing sites. The orientation of $\mathrm{N}$ domains is also dependent on the arrangement of the D1 ring, resulting in six possible patterns for each $\mathrm{N}$ domain and in a total of twelve modi (figure 2.11 b). Since the opposing $\mathrm{N}$ domain pair leads to patterns in excess by the factor of two, the resulting total number of possible patterns is nine. Three of these were observed by cryo-EM studies and were named as State I, II and III (IIla and IIIb), respectively (figure 2.11 b and c). 50.53

A possible explanation for observing only three states could be that the $\alpha$-SNAP/SNARE spire favours a certain position between the $\mathrm{N}$ domains. In fact, it exhibits a tendency towards chains $\mathrm{E}$ and $\mathrm{F}$ of the $\mathrm{N}$ domains which are in the raised position of the split washer $\mathrm{D} 1$ structure. This favoured orientation could facilitate a possible interaction with the pore loops of D1 domains $E$ and $\mathrm{F}$ with the SNARE/SNAP subcomplex. State III was divided into two subclasses, states IIla and IIIb, due to a difference in the spire orientation, but not in the $\alpha$-SNAP/N domain interaction mode (figure $2.11 \mathrm{c}$ ).

To summarise, the $20 \mathrm{~S}$ (super)complex was observed in four different states and could be distinguished by the $\alpha$-SNAP/N domain interacting modi and the orientation of the spire. The $\alpha$-SNAP/SNARE subcomplex and the basement D2 ring did not show any differences in these states. 50,53 
a

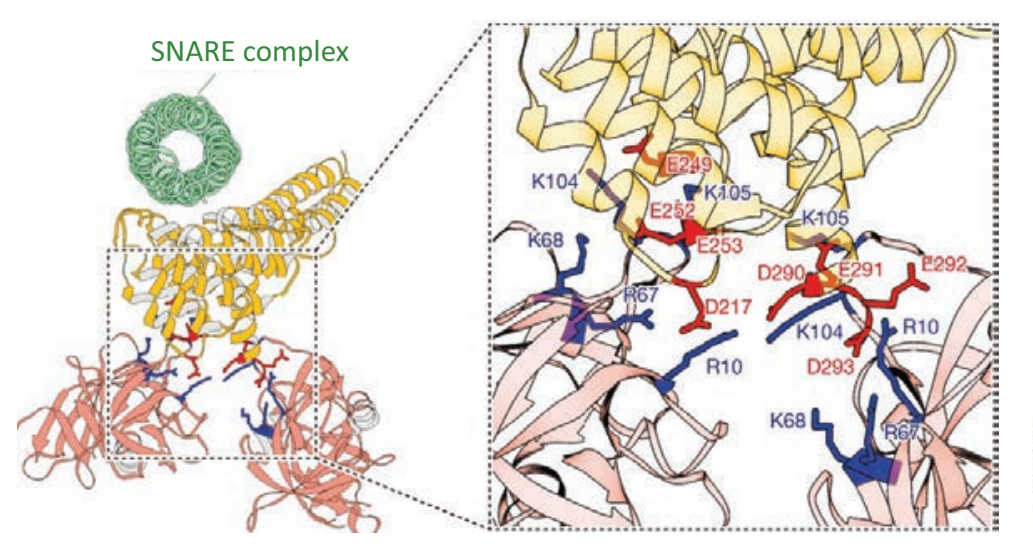

b $20 \mathrm{~S}$ (super)complex NSF $+4 \alpha-S N A P$

C

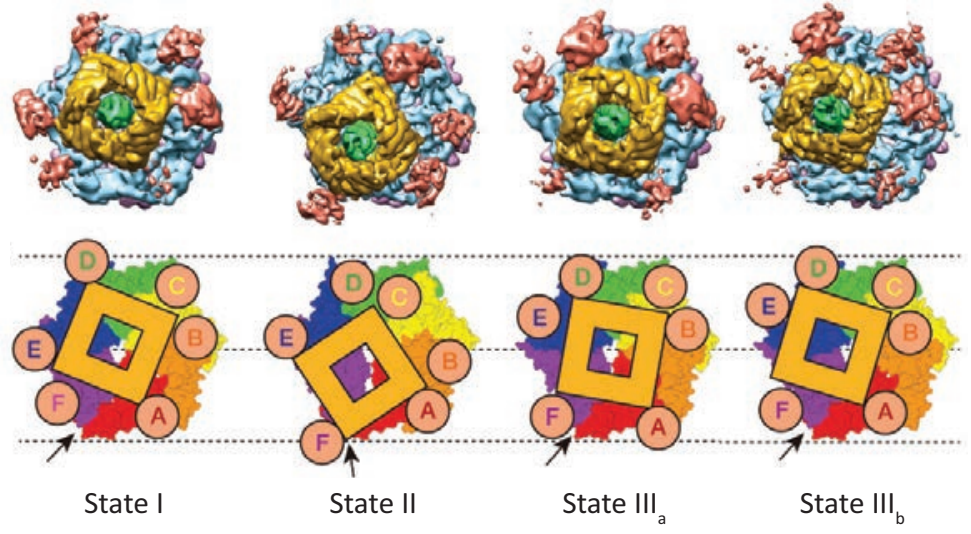

Figure 2.11: (a) Interactions between NSF N domain and $\alpha$-SNAPs with binding site I and II of $\alpha$-SNAP highlighted in red and binding site of $\mathrm{N}$ domain highlighted in blue. Figure modified from ZHAO \& BRUNGer. [53] (b) Possible states of the $20 \mathrm{~S}$ (super)complex regarding $\mathrm{N}$ domain $/ \alpha$-SNAP interaction patterns. Figure modified from ZHAO et al. [50] Adapted by permission from Macmillan Publishers Ltd: Nature, reference [50], copyright ( 2015 . (c) Four observed states of the $20 \mathrm{~S}$ (super)complex as sharpened maps (top) and schematically (bottom). The black arrow highlights the opening between subdomains $A$ and F. Figure modified from ZHAO \& BRUNGER. 53.

The recent $20 \mathrm{~S}$ (super)complex structure also reveals new insights into the interaction between $\alpha$-SNAPs and the SNARE complex within the subcomplex (figure 2.12). The lefthanded SNARE complex is surrounded by the right-handed $\alpha$-SNAPs, as shown previously (MARZ et al. ${ }^{[64]}$ ). Since the SNARE complex is a heterotetramer, it exhibits a pseudo four-fold symmetry. So it is not surprising that four $\alpha$-SNAPs surround the complex in a four-fold rotational manner. It is assumed that the $C$-terminal part of the SNARE complex is orientated towards the top of the structure due to the transmembrane domains. This is supported by the identified position of the membrane-attachment site of $\alpha$-SNAPs likewise towards the top of the spire (figure $2.12 \mathrm{a}$ ). The binding sites are based on electrostatic interactions, just as $\alpha$-SNAP/N domain binding, consisting of positively charged residues of $\alpha$-SNAP (K122, 
K163, K203 and R239) and negatively charged residues on the surface of the SNARE complex (figure $2.12 \mathrm{~b}$ ). The distinct residues were identified by mutagenesis studies and support the previously published results by MARZ et al. ${ }^{64}$. Residues $\mathrm{K} 122$ and $\mathrm{K} 163$ are orientated closely to the zero layer of the SNARE complex and residues K203 and K239 are localised at the $C$-terminal region of $\alpha$-SNAP. Mutation of negatively charged residues on the surface of $\alpha$-SNAP (E38A, E40A, E43A, and D80A) showed only a slight effect on the non-membrane bound disassembly reaction. This supports the hypothesis of the positively charged $\alpha$-SNAP surface binding to the negatively charged SNARE complex surface (figure $2.12 b-d$ ). 50,53 ]
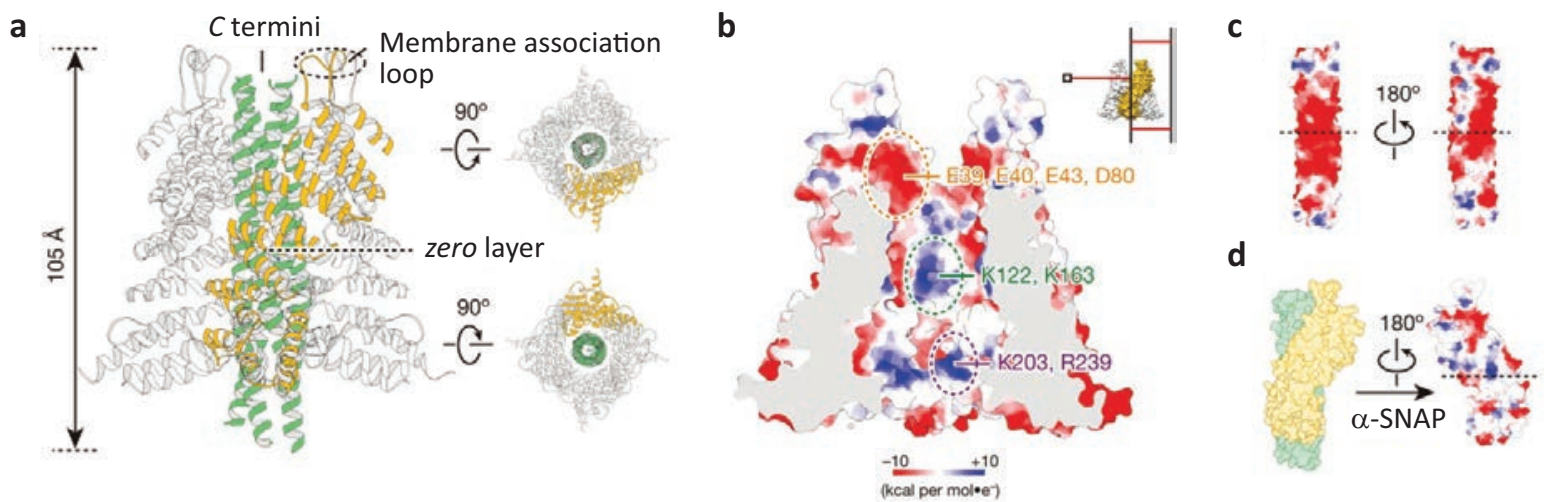

Figure 2.12: (a) Interactions between $\alpha$-SNAPs (yellow) and SNARE complex (green) as ribbon representation based on state I of the $20 \mathrm{~S}$ (super)complex. (b) Electrostatic potential of $\alpha$-SNAP barrel surface which interacts with the SNARE complex. Positively charged residues interacting with the SNARE complex are highlighted in green (K122, K163) and purple (K203, R239). (c) Electrostatic potential of the SNARE complex surface with the zero layer indicated by a dotted line. (d) Electrostatic potential of the surface of one $\alpha$-SNAP molecule. Figures modified from ZHAO et al. [50] Adapted by permission from Macmillan Publishers Ltd: Nature, reference [50], copyright (C) 2015.

With a different SNARE complex composition (VAMP-7 instead of Synaptobrevin-2), the so-called V7-20 S complex showed another $\alpha$-SNAP stoichiometry. In this case, only two $\alpha$-SNAP molecules are present in the structure. It was concluded that NSF is also able to disassemble the SNARE complex with fewer cofactors by aligning its flexible $\mathrm{N}$ domains. On the other hand, more than four $\alpha$-SNAPs are rather unrealistic due to sterical hindrance. 50,53

NSF as well as SNAPs are highly conserved and are able to disassemble various SNARE complexes. [3] The binding between NSF/ $\alpha$-SNAP as well as $\alpha$-SNAP/SNARE complex is based on electrostatic interactions. These are less specific than hydrogen bonds, explaining the non-specificity of the $\alpha$-SNAP/NSF system and its ability to disassemble a variety of SNARE complexes in the secretory pathway. ${ }^{[50}[53]$ 


\subsubsection{Mechanism of NSF $/ \alpha$-SNAP-Mediated SNARE Disassembly}

Based on the recent cryo-EM structures, a possible mechanism for the NSF/ $\alpha$-SNAP-mediated SNARE disassembly reaction was suggested (figure 2.13). 50 53] Firstly, up to four $\alpha$-SNAPs bind to the cis-SNARE complex (figure $2.13 \mathrm{a}$ and $\mathrm{b}$ ). The number of $\alpha$-SNAP molecules depends on the SNARE complex composition. Secondly, ATP-bound NSF binds to the $\alpha$-SNAP/SNARE subcomplex leading to the formation of the $20 \mathrm{~S}$ (super)complex (figure $2.13 \mathrm{c}$ ). Due to the binding interaction, the NSF D1 and D2 domains are tightened like a compressed spring. The four observed states of the $20 \mathrm{~S}$ (super)complex suggest that the whole system applies a torsional force to uncoil the SNARE complex while changing the states. ATP hydrolysis triggers the conformational changes of the $\mathrm{N}$ domains, which is transferred via $\alpha$-SNAPs. Lastly, in ADP-bound NSF, two $\mathrm{N}$ domains are pointed downwards and an opening occurs within the D1 ring, which both may function as an exit for the individual SNARE proteins (figure $2.13 \mathrm{~d}$ ). A pore translocation mechanism is unlikely due to the fact of the missing pore loops in the D2 domains. After the release of the SNARE proteins and $\alpha$-SNAPs, nucleotides are exchanged and the cycle can start from the beginning. 50 ]

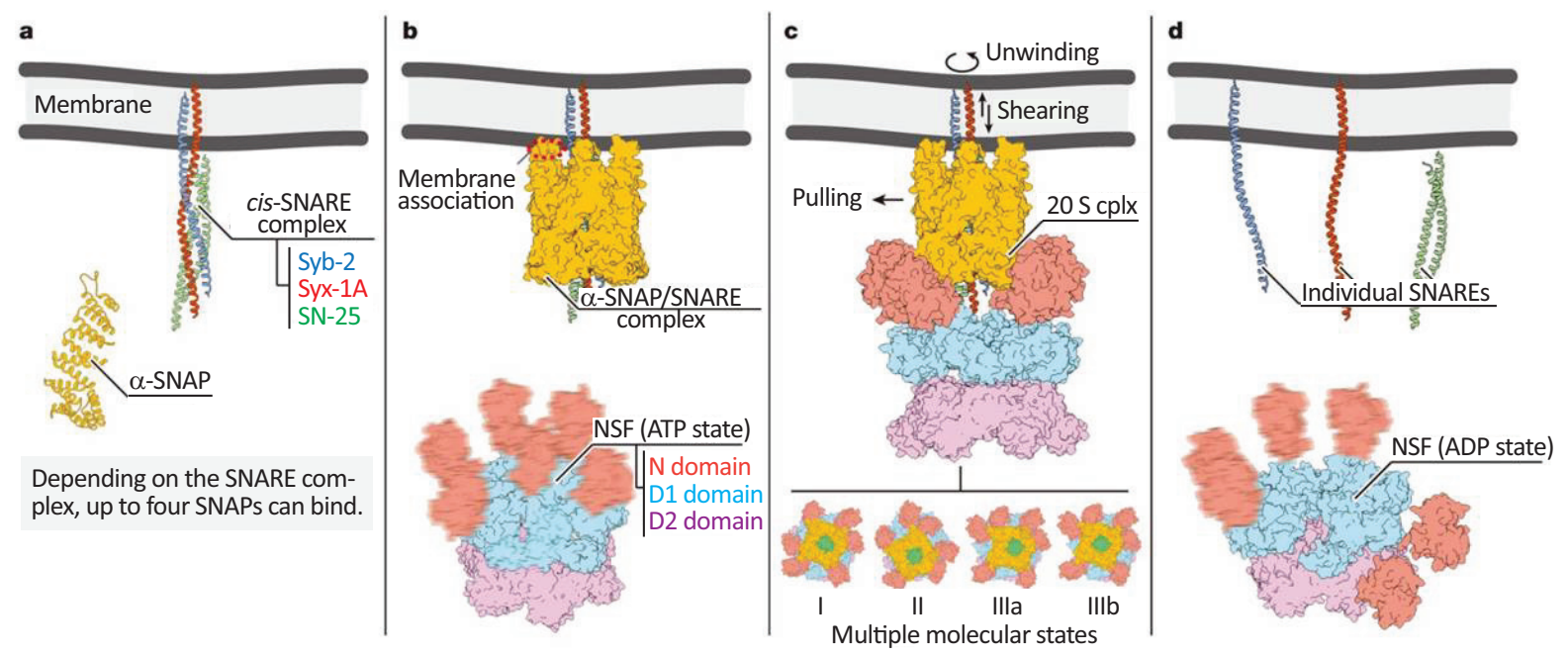

Figure 2.13: $(\mathbf{a}-\mathbf{d})$ Suggested mechanism of NSF/ $\alpha$-SNAP-mediated SNARE disassembly reaction consisting of four steps. $\mathrm{N}$ domains are blurred to indicate flexibility ( $\mathbf{b}$ and $\mathbf{d}$ ). Figure adapted from ZHAO et al.[50] Adapted by permission from Macmillan Publishers Ltd: Nature, reference [50], copyright (c) 2015.

\subsection{Complexin - A Regulatory Protein of SNARE-Mediated Membrane Fusion}

Complexins are a family of evolutionarily conserved proteins which are relatively small (15 $20 \mathrm{kDa}$ ), hydrophilic and mainly found in the neuronal cytosol. They were firstly identified in 
1995 by TAKAHASHI et al. ${ }^{65]}$ screening a murine brain cDNA library, by ABE et al.66] due to constant copurification with the neuronal SNARE complex, as well as by SÜDHOF et al. 67 due to their binding ability to the neuronal SNARE complex. [68 69] ABE et al. ${ }^{[66]}$ named them first "Synaphins" from the latin synaphia, meaning "maintenance of the same rhythm". But the name "Complexins" suggested by SüDHOF et al. $\frac{67}{}$, due to the binding properties to the SNARE complex, was generally accepted.

The first identified isoforms, Complexin I (Cpx I) and Complexin II (Cpx II) are unusually highly conserved which is an indication for their importance. Cpx I exhibits $97 \%$ sequence consensus in human and murine orthologs, and Cpx II is even identical in humans and murines. 67, 69 [70] In addition, between $\mathrm{Cpx}$ I and Cpx II an 80 - 86 \% sequence homology shows a close relationship of these two isoforms. ${ }^{65]}$ 67 [6] However, they exhibit only $24-28 \%$ sequence homology to Complexin III (Cpx III) and Complexin IV (Cpx IV). Consequently, the latter two set up a second subfamily. The main difference between the $\mathrm{Cpx} \mathrm{I/II-} \mathrm{and} \mathrm{Cpx} \mathrm{III/IV-subfamilies} \mathrm{constitute}$ their $C$-terminal domains. In the case of $\mathrm{Cpx}$ III/IV, an extension for lipidation can be found at the $C$-terminal ends. ${ }^{69}$ 10]. All Complexin isoforms are especially expressed in the central nervous system $65-67[69]$, with Cpx IV as an exceptional case, because its expression is mainly restricted to retinal ribbon synapses. $\frac{69}{70}$ In addition, Cpx IV exhibits a lower binding affinity to the SNARE complex than the other three isoforms. For its function, membrane anchoring is essential which leads to higher concentration at the active sites and, therefore, compensation of the lower binding affinity. Compared to mammals, in invertebrates a smaller number of Complexin isoforms is expressed. In a sequence-specific manner, they are related to the $\mathrm{Cpx} \mathrm{I/II}$ subfamily but additionally exhibit a $C$-terminal extension containing a farnesylation motif like Cpx III/IV. It seems that Complexins in mammals are functionally more specialised and evolve from an ancestral protein with a more general function. 69 70

Structurally, Complexins comprise four domains within their sequences. In the case of Complexin I, they are distributed as follows (figure 2.14): a $N$-terminal domain $(1-28)$, an accessory $\alpha$-helix $(29-47)$, a central $\alpha$-helix $(48-70)$, and a $C$-terminal domain $(71-134)$. 686961 The region between amino acid residues $29-86$ forms an $\alpha$-helical structure but overall Complexins exhibit no tertiary structure. The central part of the $\alpha$-helix was identified as the binding motif for the SNARE complex. Complexin $I$ is binding fast and with high affinity $\left(K_{\mathrm{d}}=10 \mathrm{nM}^{[72]}\right)$ to the ternary, neuronal SNARE complex, but no binding was observed to individual SNARE proteins. $68,69,72,74$

The research interest for Complexins especially arose when they were suggested to be involved in neuronal diseases, such as schizophrenia and Huntington's disease. ${ }^{74}$. Although, this correlation was soon questioned ${ }^{75}$, the interest did not diminish within the research field of Complexin function. 


Cpx I:
\begin{tabular}{|c|c|c|c|}
\hline \multicolumn{2}{|c}{29} & \multicolumn{2}{c}{70} \\
\hline $\begin{array}{c}N \text {-terminal } \\
\text { domain }\end{array}$ & $\begin{array}{c}\text { Accessory } \\
\alpha \text {-helix }\end{array}$ & $\begin{array}{c}\text { Central } \\
\alpha \text {-helix }\end{array}$ & C-terminal domain \\
\hline
\end{tabular}

Figure 2.14: Domain structure of Complexin I $(\mathrm{Cpx} \mathrm{I})$ with the $N$-terminal domain $(1-28)$, the accessory $\alpha$-helix $(29-47)$, the central $\alpha$-helix $(48-70)$, and the $C$-terminal domain $(71-134)$.

\subsubsection{Structure of the Complexin/SNARE Complex}

The crystal structure of the Complexin/SNARE complex revealed that Complexin binds to the groove between Synaptobrevin-2 and Syntaxin-1A in an antiparallel orientation and with a $1 / 1$ stoichiometry (figure $2.15 \mathrm{a}-\mathrm{c}$ ). This interaction stabilizes the interface between the Sybnaptobrevin-2/Syxntaxin-1A helices, but no further structural changes of the ternary complex could be observed. 68 69 74 The distinct interacting positions were found to be in the central $\alpha$-helix of Complexin (48 - 70), and Syntaxin-1A (214 - 232) and Synaptobrevin-2 $(47-68)$ amino acid residues close to the zero layer (figure $2.15 \mathrm{~d}-\mathrm{f}$ ). One lysine residue (K69), two tyrosine residues (Y52 and Y70) as well as three arginine residues (R48, R59, and R63) of Complexin were identified to be crucial for binding. Specifically, the lysine residue (K69) and the tyrosine residue (Y70) of Complexin interact with the aspartic acid residue (D218) of Syntaxin-1A (figure 2.15 d). In addition, the arginine residues (R59 and R63) of Complexin interact with the aspartic acid residue (D57) of Synaptobrevin-2 (figure 2.15 e). Finally, the tyrosine residue (Y52) of Complexin interacts with the aspartic acid residue (D64) of Synaptobrevin-2 as well as the arginine residue (R48) of Complexin with the aspartic acid residues (D65 and D68) of Synaptobrevin-2 (figure 2.15 f). To summarise, the Complexin binding to the SNARE complex is based on electrostatic interactions comparable to the $\alpha$-SNAP binding. 74

For the crystal structure analysis, the Complexin fragment $(26-83)$ was used but only amino acid residues $(32-72)$ could be resolved in the structure. This indicated that the $\mathrm{N}$-terminal part as well as the $\mathrm{C}$-terminal part are highly flexible and exhibit no contribution to binding. ${ }^{74]}$ Complexins bind to the ternary, neuronal SNARE complexes, but their function on neurotransmitter release remains controversially discussed.

\subsubsection{Controversially Discussed Function of Complexin}

Due to a large number of studies in various model systems concerning the function of Complexin, which have led to controversial results, it is generally accepted that Complexin comprises a dual function in neurotransmitter release. Though, it is only a small molecule, an inhibitor as well as an activator can be found. The inhibitor function is assumed to be needed for spontaneous neurotransmitter release and the facilitatory function for $\mathrm{Ca}^{2+}$-triggered release, but the molecular mechanisms of this dual function are still not understood. 68 69] It could be shown, 

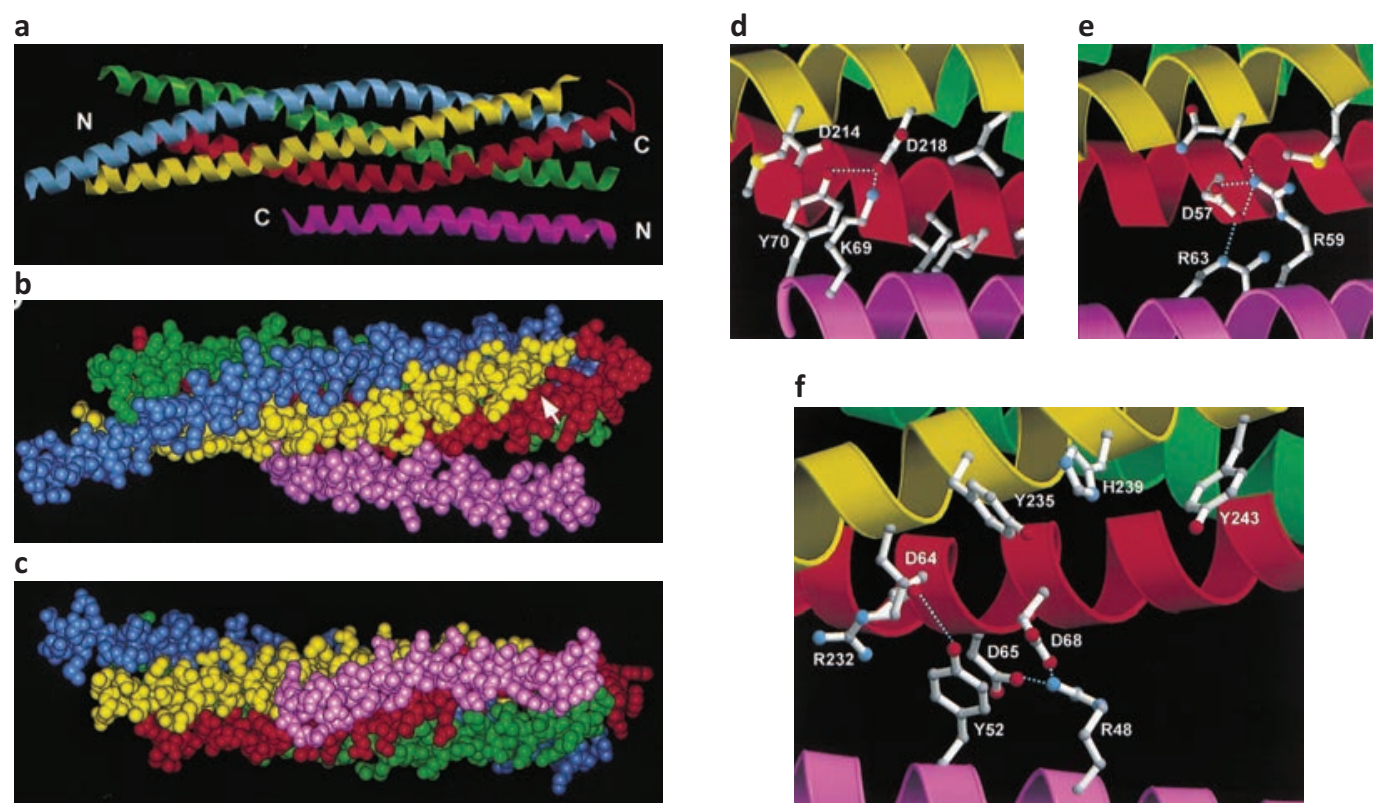

Figure 2.15: (a) Crystal structure of the Complexin/SNARE complex ( $\mathrm{pdb}$ code 1kil) as ribbon diagram and ( $\mathbf{b}$ and $\mathbf{c}$ ) as space filling models in two different views. The following color code is used: yellow = Syntaxin-1A, red = Synaptobrevin-2, blue/green $=$ SNAP-25, pink $=$ Complexin . $(\mathbf{d}-\mathbf{f})$ Close-ups of the binding interface between Complexin, Syntaxin-1A, and Synaptobrevin2. Figures used from CHen et al. ${ }^{74}$ Reprinted from reference [74], Copyright (C) 2002, with permission from Elsevier.

that the domains of Complexin take different parts in regulating neurotransmitter release. The central $\alpha$-helix as binding site as well as the binding to the SNARE complex are required for the overall function of Complexin. The accessory $\alpha$-helix was shown to contribute to the inhibitory function, whereas the $\mathrm{N}$-terminal domain facilitates $\mathrm{Ca}^{2+}$-triggered release. 68 69 71 16] Lastly, the $C$-terminal domain is suggested to target Complexin to the active site. ${ }^{68}[76$

Two mechanistic models have been proposed so far: The so-called 'fusion clamp' model explains the inhibitory function, whereas the fusion facilitatory function was suggested to be based on regulating the vesicle fusogenicity. $\frac{68}{60}$

\section{The 'fusion clamp' model}

The 'fusion clamp' model was suggested and investigated in detail by RoTHMAN et al. $77-82]$ It describes the inhibitory effect of Complexin to be based on the arrest of primed synaptic vesicles at the nerve terminal which should be released only upon $\mathrm{Ca}^{2+}$ influx. In other words, Complexin is responsible for 'clamping' the primed vesicles and prevents premature, spontaneous fusion. It is thought that Complexin first binds to the incompletely zippered SNARE complex via its central $\alpha$-helix $(48-70)$ and that its accessory $\alpha$-helix $(29-47)$ can compete with the C-terminal part of Synaptobrevin-2 to prevent full zippering. 68 69 67-79] GIRAUDo et al. developed a 'superclamp' Complexin ( $\mathrm{scCpx}$ ) on the basis of sequence similarities between the accessory $\alpha$-helix of Complexin and the $C$-terminal part of the Synaptobrevin-2 SNARE 
a

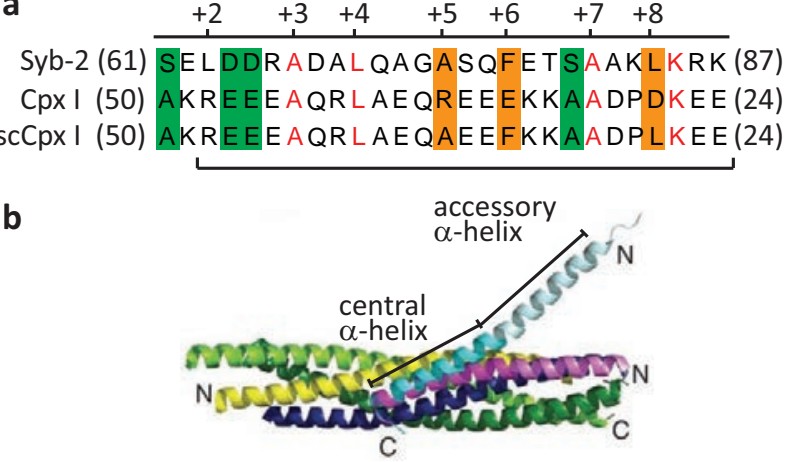

C

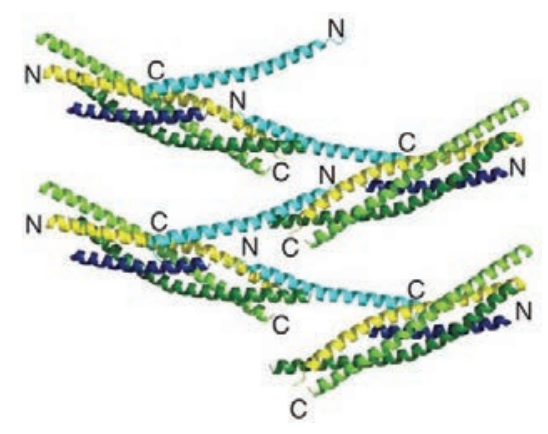

Figure 2.16: (a) Sequence alignment of the C-terminal SNARE motif of Synaptobrevin-2 (residues $61-87$ ) and Complexin I accessory $\alpha$-helix (residues $29-47$, antiparallel orientation). Sequence consensuses are highlighted in red, sequence similarities in green, and sequence adaption for 'superclamp' Complexin in orange. Figure modified from GiRAudo et al.79] From reference [79]. Reprinted with permission from AAAS. (b) Overlay of crystal structures of 'superclamp' Complexin/truncated SNARE complex (prefusion, pdb code 3rk3) and wild-type Complexin/SNARE complex (postfusion, pdb code $1 \mathrm{kil}$ ). The following color code is used: blue $=$ Synaptobrevin-2, yellow $=$ Syntaxin-1A, light and dark green $=$ SNAP-25, light and dark pink $=$ wild-type Complexin, and light blue and grey $=$ 'superclamp' Complexin. (c) Side view of the 'zigzag' topology formed by 'superclamp' Complexin/truncated SNARE complex. Figures modified from KüMMEL et al. [1] Adapted by permission from Macmillan Publishers Ltd: Nature Structural and Molecular Biology, reference [81], copyright (C) 2011.

motif (layer $+1-+8)$. In this Complexin mutant, three additional hydrophobic residues (D27L, E34F, R37A) were introduced to regenerate suitable interaction layers $(+5,+6$, and +8$)$ within the SNARE coiled-coil (figure 2.16 a). It was predicted to interact with the incompletely zippered SNARE complex by binding to the Syntaxin-1A/SNAP-25 interface at the C-terminal part of the SNARE motifs. Indeed, in vitro cell-cell fusion assays showed an increase in clamping efficiency. ${ }^{[79]}$ However, on the other hand, in vivo experiments exhibit controversial results. ${ }^{76}$.83]

KÜMMEL et al. were able to co-crystallize 'superclamp' Complexin with a truncated SNARE complex (figure $2.16 \mathrm{~b}$ ). The crystal structure of Complexin I (26 - 83 D27L, E34F, R37A) and SNARE $\Delta 60$ complex consisting of Synaptobrevin-2 (29-60), Syntaxin-1A (10 - 82) and SNAP-25 $(10-82,141-203)$ showed an intermolecular interaction. The central $\alpha$-helix of Complexin was bound to one SNARE complex, whereas its accessory $\alpha$-helix was bound to the $C$-terminal part of the SNARE motif of a second SNARE complex, and thereby, forming a 'zigzag' topology (figure 2.16 c). [1] Nevertheless, recently published NMR studies by TRIMBUCH et al. were not able to confirm the structure of the 'superclamp' Complexin/truncated SNARE complex in solution. Additionally, there is a bare possibility that the charged wild-type accessory $\alpha$-helix is really inserting into the hydrophobic Synaptobrevin- 2 binding site of the SNARE complex. 68 83]

KRISHNAKUMAR et al. further tested that Complexin has to be switched from open (prefusion) 
to closed (postfusion) conformation to enable Synaptotagmin $/ \mathrm{Ca}^{2+}$-triggered fusion. In the open or prefusion state, the 'superclamp' accessory $\alpha$-helix is able to bind to a second SNARE complex and blocks the Synaptobrevin-2 binding site. ${ }^{80}$. On the other hand, in the closed or postfusion conformation, the accessory $\alpha$-helix is more closely located to the SNARE complex to which its central $\alpha$-helix is bound. 74 [8] The switching process was monitored by FRET experiments between donor dye (stilbene) at residue 193 of SNAP-25 and acceptor dye (bimane) at residue 38 of Complexin. Zippering of Synaptobrevin- 2 until layer +4 was found to be crucial for switching between the two Complexin conformations. With truncated Synaptobrevin-2 mutants (Syb-2 65, Syb-2 69, Syb-2 73, and Syb-2 77), a stepwise zippering was imitated. Specifically, Synaptobrevin-2 residues D64, D65, and D68 have to be zippered and have to interact with the central $\alpha$-helix of Complexin to change the position of the accessory $\alpha$-helix. In addition, a Synaptobrevin-2 mutant lacking these three aspartic acid residues (D64A, D65A, and D68A) fully zippers into the ternary SNARE complex, but leaves Complexin in its open conformation. On the other hand, the truncated Synaptobrevin-2 mutants for stepwise zippering revealed that the aspartic acid residues do not only have to be present but also have to be zippered to act as a conformation switch for Complexin. Only the proceeding zippering of the ternary SNARE complex can switch the conformation of Complexin, and consequently enable the interaction with Synaptotagmin $/ \mathrm{Ca}^{2+}$ for triggered fusion ${ }^{80}$ (see chapter 2.7.3).

As expected, Complexin ( $\mathrm{Cpx}$ I 48 - 134) binding to the SNARE complex containing Synaptobrevin-2 (D64A, D65A, D68A) mutant exhibits lower affinity $\left(K_{\mathrm{d}}=620 \pm 110 \mathrm{nM}^{[80]}\right)$ than to the wild-type ternary SNARE complex $\left(K_{\mathrm{d}}=43 \pm 7 \mathrm{nM}^{[82]}\right)$. The latter still shows lower affinity compared to full-length wild-type Complexin I $\left(K_{d}=10 \mathrm{nM}^{[72]}\right)$.

To summarise, the hypothesis of the underlying mechanism of Complexin's fusion clamp function is still hotly debated. The results obtained by FRET, X-ray, NMR and isothermal titration calorimetry (ITC) to investigate the interaction between the accessory $\alpha$-helix of Complexin and the SNARE complex are inconsistent so far. It is generally accepted that the accessory $\alpha$-helix contributes to the inhibitory function of Complexin, but despite a huge number of studies and different hypotheses, no satisfying coincidence of the underlying mechanism has been found to date. $68,69,82,83$

\section{The fusion facilitatory model}

The Reduction of evoked neurotransmitter release in several knock-out studies of Complexin indicated its additional facilitatory function. 68 60 However, in comparison to the 'clamp model', the underlying mechanism is less investigated. Initially, the hypothesis was proposed that Complexin acts as a promoter by stabilising the SNARE complex due to the binding of its central $\alpha$-helix. ${ }^{74]}$ Detailed structure-function studies revealed that the $N$-terminal domain 


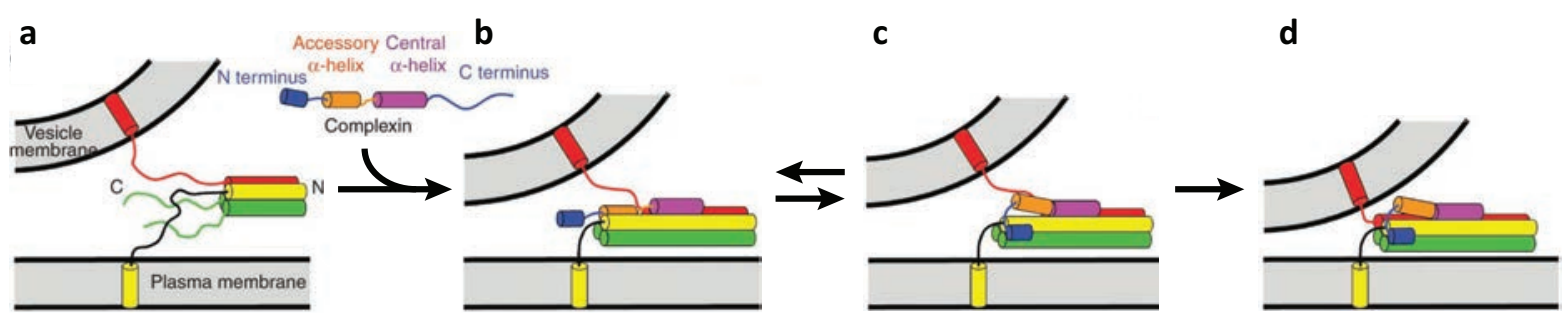

Figure 2.17: Hypothesised model for facilitatory function of Complexin's $N$-terminal domain and combination with inhibitory function of the accessory $\alpha$-helix. (a) SNARE complex zippering starts at the $N$-terminal part of SNARE motifs. (b) Complexin binds to the SNARE complex via its central $\alpha$-helix and stabilizes SNARE assembly. On the other hand, it possibly blocks Synaptobrevin-2 binding site by its accessory $\alpha$-helix. This corresponds to the 'clamped' state. (c) The inhibition is released by the interaction of Complexin's $N$-terminal domain with the $C$-terminal part of SNARE complex (exact location of interaction not clear, only schematic suggestion) and/or by Synaptotagmin- $1 / \mathrm{Ca}^{2+}$ (not shown). (d) Full assembly of SNARE complex and possible stabilisation by Complexin's $N$-terminal domain to regulate vesicle fusogenicity. Figure modified from XUE et al. [84] Adapted by permission from Macmillan Publishers Ltd: Nature Structural and Molecular Biology, reference [84], copyright (c) 2010.

(residues $1-26$ ) is crucial for the activating function and it was speculated to interact with the SNARE complex or with lipid membranes. 68 71 83 84] Indeed, NMR studies and fluorescence spectroscopy revealed that the $N$-terminal domain of Complexin interacts with the $C$-terminal end of the SNARE complex. ${ }^{84}$ By this interaction, it was suggested that Complexin stabilises the $C$-terminal part of the SNARE complex upon zippering, and thereby supports the SNARE machinery to provide the energy needed for fusion. Consequently, Complexin influences the vesicle fusogenicity and regulates spontaneous as well as $\mathrm{Ca}^{2+}$-triggered release. Fusogenicity describes the height of energy barrier which a primed vesicle has to overcome before membrane fusion can occur, and can be measured by $\mathrm{Ca}^{2+}$-independent, induced vesicle fusion through hypertonic solutions. Additionally, interaction of the $N$-terminal end of Complexin to the SNARE complex may promote the removal of the inhibitory function by the accessory $\alpha$-helix (figure 2.17). It could be shown, that the vesicle fusogenicity is increased possibly at a step prior to triggering by $\mathrm{Ca}^{2+}$ influx, due to the fact that Complexin facilitates $\mathrm{Ca}^{2+}$-triggered release even in the absence of Synaptotagmin-1. As a result, the facilitatory function of Complexin is suggested to act together with Synaptotagmin-1, but the latter was not essential for this function. 68 84

Overall it can be summarised, that the dual function of Complexin is based on the different contributions of its specific domains. The central $\alpha$-helix and the $N$-terminal domain feature the facilitatory function by stabilisation of the SNARE complex assembly, increase in the vesicle fusogenicity, and maybe interference with the accessory $\alpha$-helix interaction. The inhibitory function or 'fusion clamp' is contributed by the accessory $\alpha$-helix through the hypothesised blockage of Synaptobrevin-2 binding site within the incompletely zippered SNARE complex and/or electrostatic repulsions with the fusing membranes. In addition, it is generally accepted 
that Complexin and Synaptotagmin-1 cooperate in some way to regulate $\mathrm{Ca}^{2+}$-triggered neurotransmitter release, which is described in the following chapter $2.7 .3,68,69,71,79,81,84$

\subsubsection{Collaborative Regulation by Complexin and Synaptotagmin}

For $\mathrm{Ca}^{2+}$-triggered neurotransmitter release, a tight regulation of the fusion machinery is necessary. Among other regulatory proteins, the key players in this part are unquestionably Synaptotagmins as $\mathrm{Ca}^{2+}$ sensors and Complexins due to their direct SNARE complex binding. Complexin function was also found to be influenced by $\mathrm{Ca}^{2+}$ concentrations which suggests an collaborative regulation by Complexins and Synaptotagmins. 3 [5 [-7]

Synaptotagmin-1 (Syt-1), as well as its closely related isoforms Syt-2 and Syt-9, function as $\mathrm{Ca}^{2+}$ sensors for the fast neurotransmitter release less than $0.5 \mathrm{~ms}$ after $\mathrm{Ca}^{2+}$ influx, which is stated as synchronous release. ${ }^{[5+7]}$ [85] Structurally, Syt-1 comprises a transmembrane domain and two $\mathrm{Ca}^{2+}$-binding domains, $\mathrm{C}_{2} \mathrm{~A}$ and $\mathrm{C}_{2} \mathrm{~B}$ which bind three and two $\mathrm{Ca}^{2+}$ ions, respectively (figure 2.18 a and $b$ ). ${ }^{5-7} 85$ 86] Within the two domains, loops at the top a $\beta$-sandwich structure exhibit five aspartic acid residues which are responsible for $\mathrm{Ca}^{2+}$ binding as well as $\mathrm{Ca}^{2+}$-dependent negatively charged phospholipid binding. 5, 7, 86] The latter induces a membrane interaction by hydrophobic residues and additional interaction of positively charged residues of

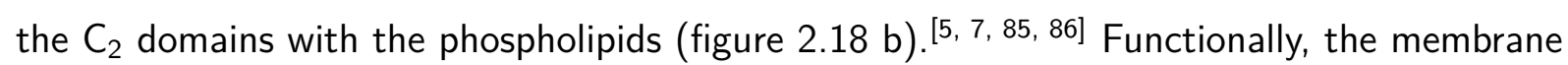
interaction was found to be crucial for Syt- 1 supporting $\mathrm{Ca}^{2+}$-dependent SNARE-mediated membrane fusion. ${ }^{785}$ Cryo-EM structures revealed that, upon $\mathrm{Ca}^{2+}$ binding, Syt-1 is able to bind two opposing membranes and at the same time pulls them together. 7.78 88. Before $\mathrm{Ca}^{2+}$ triggering, the negatively charged aspartic acid residues of the $\mathrm{Ca}^{2+}$ binding region were suggested to induce repulsive forces towards the phospholipids of the membrane, and thereby inhibit membrane fusion until the $\mathrm{Ca}^{2+}$ signal. ${ }^{7}$ Consequently, it was hypothesised that Syt-1

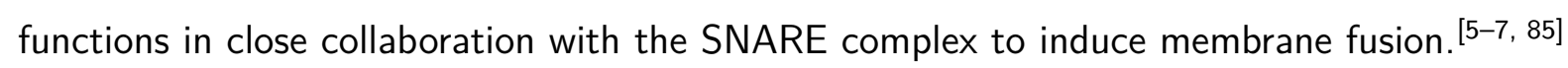

Syt-1 directly binds to the SNARE complex by positively charged residues localised at the side of the $C_{2} B$ domain $\beta$-sandwich (figure $2.18 \mathrm{~b}$ and $\mathrm{c}$ ). The binding site for Syt-1 on the surface of the SNARE complex was found to be localised at SNAP-25 helices in the middle of the complex. ${ }^{789]}$ This implies that the Syt-1 and Cpx I binding sites are not competitive and both proteins can bind concurrently. Upon binding to the SNARE complex surface, the $\mathrm{C}_{2} \mathrm{~B}$ domain would be perfectly located for binding to the opposing membranes upon $\mathrm{Ca}^{2+}$ influx. These interactions could induce repulsive forces between the accessory $\alpha$-helix of Complexin and the membranes which may be the explanation for Syt-1 induced removal of Complexin's 'clamp' function. 7

The following model was proposed by RIZO \& $\mathrm{XU}^{[7}$ including the cooperation between SNARE complex, Syt-1, Complexin and $\mathrm{Ca}^{2+}$-triggering (figure $2.18 \mathrm{c}$ ): Prior to the $\mathrm{Ca}^{2+}$ signal, the 

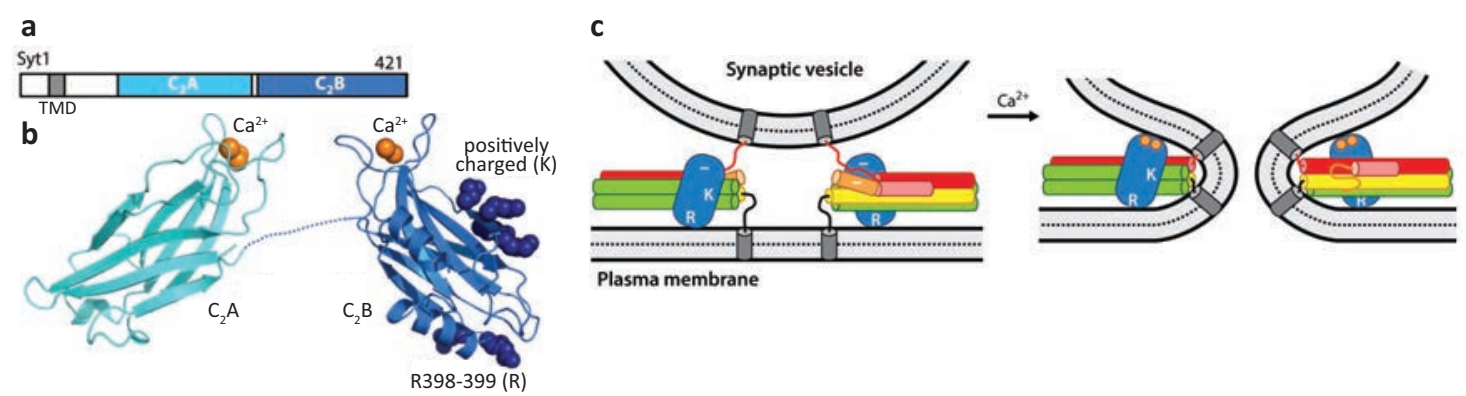

Figure 2.18: (a) Domains and (b) crystal structure of $\mathrm{Ca}^{2+}$-bound Synaptotagmin-1 (Syt-1, pdb code 1 byn and $1 \mathrm{k} 5 \mathrm{w}$ ) including a transmembrane domain (TMD) and two $\mathrm{Ca}^{2+}$ binding domains $\mathrm{C}_{2} \mathrm{~A}$ (light blue) and $\mathrm{C}_{2} \mathrm{~B}$ (dark blue). $\mathrm{Ca}^{2+}$ ions are shown in orange. Additionally, positively charged residues ( $\mathrm{K}$ and $\mathrm{R}$ ) of $\mathrm{C}_{2} \mathrm{~B}$ domain are highlighted in dark blue. (c) Hypothesised model for cooperation between SNARE complex, Syt-1 and $\mathrm{Cpx}$ triggered by $\mathrm{Ca}^{2+}$ influx. Figure modified from RIZO \& XU. 7$]$

SNARE complex is incompletely zippered with bound Complexin preventing further zippering by its accessory $\alpha$-helix. Simultaneously, Syt- 1 is bound to the SNARE complex and supports Complexin's 'fusion clamp' by electrostatic repulsion between its negatively charged $\mathrm{Ca}^{2+}$ binding residues of the $\mathrm{C}_{2} \mathrm{~B}$ domain and the negatively charged accessory $\alpha$-helix of Complexin (figure $2.18 \mathrm{c}$, left). As soon as $\mathrm{Ca}^{2+}$ concentration increases, Syt- 1 changes to the $\mathrm{Ca}^{2+}$-bound state and membrane attachment to phospholipids brings the opposing membranes in close proximity. By this interaction, Complexin's 'clamp function' of the accessory $\alpha$-helix is removed due to sterical hindrance and zippering of the $C$-terminal part of the SNARE complex can proceed. Consequently, the opposing membranes are brought in close proximity by a cooperation of Syt-1 and SNARE complex membrane interactions which increases the provided energy for membrane fusion. ${ }^{[7]}$

The recently published crystal structure of the Syt-1-SNARE complex revealed three interacting regions between the SNARE complex and the $C_{2} A$ and $C_{2} B$ domains of Syt- 1 (figure 2.19 a and b). ${ }^{85}$. The first region was shown to be most important for $\mathrm{Ca}^{2+}$-triggered neuronal exocytosis and comprises mainly electrostatic interactions between SNAP-25, Syntaxin-1A and $C_{2} B$ domain of Syt- 1 . In more detail, this region was subdivided into two subregions: Subregion I exhibits SNAP-25 residues E37, K40, N159, M163, and D166 interacting with Syt-1 residues E295, K297, N336, and Y338; subregion II exhibits SNAP-25 residues D51, E52, and E55, Syntaxin-1A residues D231, E234, and E238 interacting with Syt-1 residues R281, K288, R398, and R399, respectively (figure 2.19 b, left). In subregion II a clear interaction between the negatively charged SNARE complex surface and positively charged Syt-1 residues was observed. The second region comprises the interaction between a $\mathrm{C}_{2} \mathrm{~B}$ domain of a second Syt-1 molecule and mainly Syntaxin-1A residues, namely residues M221, E224, and E228 of Syntaxin-1A interacting with R281, T285, A286, K288, and R398 of Syt-1 (figure 2.19 b, middle). Interestingly, the third region partially overlaps with the Complexin binding region to 
the SNARE complex (chapter 2.7.1): Syntaxin-1A residue D218 and Synaptobrevin-2 residue D57 interact with R199 and R233 of Syt-1's $C_{2}$ A domain (figure 2.19 b, right). ${ }^{85}$

a

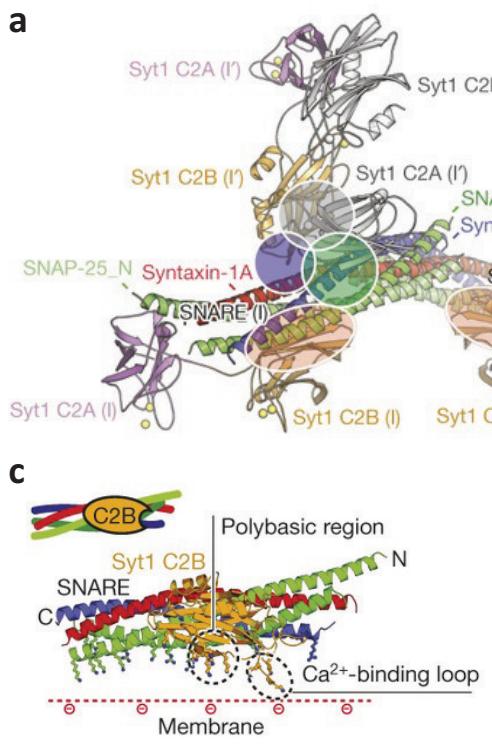

b

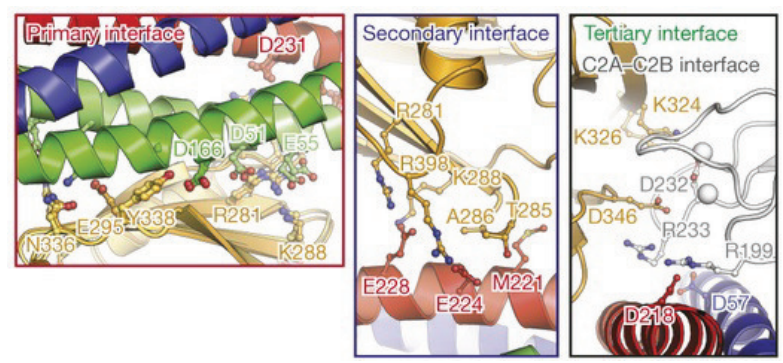

d Primed pre-fusion state
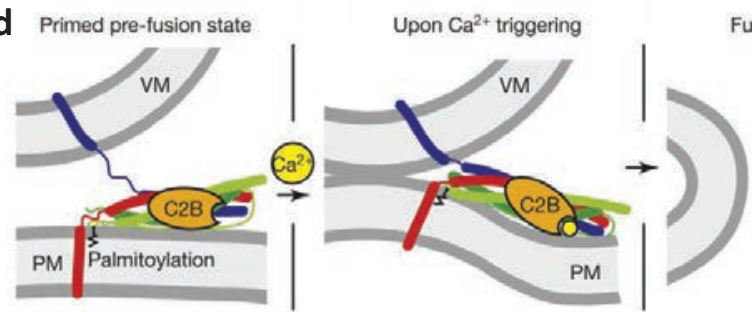

Fusion pore formation

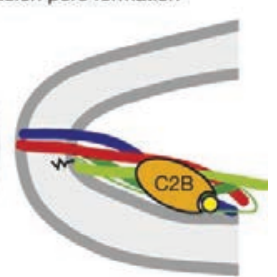

Figure 2.19: (a) Crystal structure of $\mathrm{Ca}^{2+}$-bound Synaptotagmin-1-SNARE complex (pdb code $5 \mathrm{ccg}$ ) showing three $\mathrm{C}_{2} \mathrm{~A}$ and $\mathrm{C}_{2} \mathrm{~B}$ domains (termed as $\mathrm{I}, \mathrm{I}$ ', and II and colored in grey, yellow and purple) and two SNARE complexes (I and II) including Synaptobrevin-2 (blue), Syntaxin-1A (red) and SNAP-25 (green). (b) Detailed view of the interacting regions termed as primary (left, red shaded), secondary (middle, blue shaded), tertiary (right, green shaded) as well as $\mathrm{C}_{2} \mathrm{~A}-\mathrm{C}_{2} \mathrm{~B}$ (right, grey shaded) interface. (c) Focus on the first interacting region between $C_{2} B$ domain of Syt-1 and the SNARE complex with positively charged side chains oriented towards suggested position of the membrane surface including negatively charged phospholipids (indicated by dotted red line). (d) Proposed model for the function of Syt-1-SNARE complex primary interface during $\mathrm{Ca}^{2+}$-triggered membrane fusion. Figure modified from ZHOU et al. ${ }^{85}$. Adapted by permission from Macmillan Publishers Ltd: Nature, reference [85], copyright (c) 2015.

Based on these results, a model of the Synaptotagmin- 1 function in $\mathrm{Ca}^{2+}$-triggered neuronal exocytosis was suggested focussing on the first interacting region of the $C_{2} B$ domain (figure 2.19 ( and $d$ ): In the primed state, the $C_{2} B$ domain of Syt-1 is already bound to a partially zippered SNARE complex without contact between the opposing membranes (figure $2.19 \mathrm{~d}$, left). Upon $\mathrm{Ca}^{2+}$ influx, $\mathrm{Ca}^{2+}$ binding induces membrane interaction of the $\mathrm{C}_{2} \mathrm{~B}$ domain in the region of the $N$-terminal part of the SNARE complex, thereby probably pulling the whole complex in that direction (figure $2.19 \mathrm{~d}$, middle). Thus, it induces membrane curvature and brings the membranes directly next to the SNARE TMDs in closer proximity to promote fusion (figure $2.19 \mathrm{~d}$, right). ${ }^{85}$.

In addition, the third interacting region including a $\mathrm{C}_{2} \mathrm{~A}$ domain of Syt- 1 is suggested to be involved in replacing the central $\alpha$-helix of Complexin from the SNARE complex due to the fact that the interacting region overlaps with the Complexin binding region. Thereby, the $C_{2} A$ domain of Syt-1 might be involved in 'unclamping' Complexin from the SNARE complex and 
in triggering complete zippering. ${ }^{85}$

The crystal structure revealed new insights into the interaction of Synaptotagmin-1 and the SNARE complex, but of course interactions with the membranes were not considered in these results. Therefore, it might be premature to abolish the first suggested model with additional membrane-bridging function of Syt-1. Anyhow, further investigations are needed to elucidate the overall mechanism of $\mathrm{Ca}^{2+}$-triggered neuronal exocytosis.

\subsubsection{Does Complexin Compete with $\alpha$-SNAP?}

Complexin binds to the groove between Synaptobrevin-2 and Syntaxin-1A helices of the SNARE complex between approximately the zero layer and the +4 layer. ${ }^{74}$. The same binding region around the middle of the SNARE complex was observed for $\alpha$-SNAP as well as specific interacting residues on the SNARE complex surface were revealed by side-specific mutation studies. [53 [64] Some of these residues binding to $\alpha$-SNAP (e.g. Syb-2 D55, D65) match or are in close proximity to the interacting residues of Complexin binding (e.g. Syb-2 D57, D64, D65, D68). 64 [74

Already in 1995, it was shown that Complexin and $\alpha$-SNAP compete for the binding to the SNARE complex in in vitro binding studies, despite no sequence homology was found. [67 In 2009 , these results were confirmed by fluorescence-based in vitro disassembly assays, in which the SNARE disassembly reaction was strongly inhibited by Complexin. Only with addition of excess $\alpha$-SNAP (1.2 eq.), the Complexin inhibition could be overcome. [59] In the same study, it was shown that $\alpha$-SNAP efficiency is distinct between soluble and membrane-bound SNARE complexes, whereas no difference in Complexin binding affinity between solution and lipid-bound complexes was observed. The authors suggested that whether $\alpha$-SNAP or Complexin binds to the SNARE complex is regulated by the membranes: Complexin may favourably bind to the trans-SNARE complex, whereas $\alpha$-SNAP exhibits a higher binding affinity to the cis-SNARE complex due to the position of its membrane-attachment site. $\frac{59}{5}$

Based on its overlapping binding site with Complexin and Synaptotagmin, $\alpha$-SNAP was proposed to interact not only with the tight cis-SNARE complex after fusion but also with the incompletely zippered trans-complex. In this context, PARK et al. were recently able to show an inhibitory effect of $\alpha$-SNAP itself on SNARE complex zippering. ${ }^{60}$ Certainly, side-specific mutations revealed a dependency not on $\alpha$-SNAP binding to the SNARE complex but on interaction of the membrane-attachment site of $\alpha$-SNAP with the membrane. Additionally, experiments with a truncated SNARE complex lacking the $N$-terminal part of Synaptobrevin-2 ( $\Delta \mathrm{N}$ complex) indicated that $\alpha$-SNAP arrests the SNARE complex zippering right in the middle of it, due to the fact that the Synaptobrevin-2 fragment (residues $49-96$ ) is not 
displaced by addition of 'full-length' Synaptobrevin-2 $(1-96)$ in the presence of $\alpha$-SNAP. On the other hand, the comparison between chromaffin granules $(\sim 350 \mathrm{~nm})$ and synaptic vesicles $(\sim 40 \mathrm{~nm})$ in these experiments showed that $\alpha$-SNAP has nearly no effect on fusion of synaptic vesicles. Hence, its impairment of SNARE zippering also depends on conditions like membrane curvature. In summary, it was shown that $\alpha$-SNAP also exhibits an inhibitory function on SNARE-mediated membrane fusion to generate primed and docked vesicles which was attributed so far only to SNARE regulatory proteins such as Complexins and Synaptotagmins. ${ }^{60}$ 



\section{Photocleavable Protecting Groups and Their Applications}

Biological systems exhibit a striking complexity of dynamic reactions and signalling cascades, which complicates investigations of these processes. Most challenging is the regulation of such systems without perturbation or contamination. 90 91 In addition, affecting multiple reaction steps simultaneously would provide fundamental insights into biological as well as chemical problems. ${ }^{91}$ In biology, investigations of intracellular pathways were performed by biochemical and chemical methods for a long time. But as soon as the used inhibitor or activator is injected into the cellular system no further control of distribution or timing is available. ${ }^{90}$ A possibility to overcome this problem is the usage of photo activatable compounds which were extensively investigated in the past decades. 90, 91] Light as an external trigger provides the advantages of high spatio-temporal precision, no contamination, and a non-invasive method. On a molecular level, two approaches were developed for this purpose: Firstly, so-called photoswitches, which upon irradiation reversibly isomerise, and secondly, photocleavable protecting groups (PPGs), which are irreversibly cleaved from the corresponding functionality upon irradiation. ${ }^{91} \mathrm{~A}$ compound protected by a PPG is also called "caged" compound and the photocleavage reaction is also stated as "uncaging". In this work, the second approach was applied and is further described in this chapter.

\subsection{General Design of PPGs and Caged Peptides/Proteins}

The design of a good PPG needs to fulfil certain criteria dependent on the application. Generally in in vivo experiments, a strong absorption at $\lambda>340 \mathrm{~nm}$ is necessary to prevent damage of biological systems upon irradiation. In addition, usage of longer wavelength provides the advantage of deeper penetration of the biological tissue. Besides a large extinction coefficient $\varepsilon$ at the corresponding wavelength, the photocleavage reaction should exhibit high efficiency or quantum yield $\Phi$ for release. The product of extinction coefficient and quantum yield $(\varepsilon \Phi)$ is proportional to the amount of photoreleased products, and therefore used to quantify the efficiency of a PPG. The reaction should be fast, without toxic side products, and the PPG needs to be stable and soluble in aqueous media. 91 92] As soon as a suitable PPG is found for the corresponding 
application, the caged compound has to be designed. The functional group responsible for the biological activity needs to be identified and covalently modified with the PPG. If a direct modification of the functionality is not possible or the activity is based on, e.g. molecular surface interactions, two possible alternative approaches can be applied: random multiple modifications at the interaction side or a single PPG in combination with triggered conformational changes. ${ }^{90}$

In the case of the caged peptide/protein design, direct modification of specific amino acid side chains is possible for Arg, Asp, Cys, Glu, Lys, Ser, Thr and Tyr via solid-phase peptide synthesis (SPPS) by introduction of the modified amino acid building block. Analogous to labeling reactions with fluorophores, the Cys side chain can be selectively modified with PPGs after peptide synthesis or expression. ${ }^{93}$.94 In addition, introduction of PPGs at the amide backbone ${ }^{[95]}$ and PPGs for light-induced backbone cleavage ${ }^{[96]}$ were reported. In general, caged

peptides are synthesised by SPPS but also expressed protein ligation (EPL) ${ }^{97}$ and genetic insertion of unnatural amino acids ${ }^{[98}$ are used to construct caged proteins. EPL provides the advantage of ligation between small synthesised peptides and larger expressed proteins but has also the disadvantage of certain sequence and modification restrictions. On the other hand, expression of proteins containing unnatural amino acids can introduce modifications at any position but is also technologically more demanding. Which method is used for constructing a caged peptide/protein depends on various factors, e.g. size and structure of the peptide/protein, injection or intracellular expression for in vivo applications. ${ }^{90]}$ In this work, SPPS was used for construction of a caged peptide.

\subsection{Instrumentations for Photocleavage Reactions}

Besides the design of a caged compound for a certain biological application, the used instrumentation for the photocleavage reaction represents a crucial point to consider. For simple experiments, a hand-held UV/vis lamp, $\mathrm{Hg}$ arc lamp or Xe flash lamp can be sufficient but often requires long irradiation times. In addition, the reaction volume needs to be taken into account, e.g. if the light energy is focused on a small area of the sample under a microscope, the photocleavage might take seconds, whereas in a cuvette the same reaction might take tens of minutes. The available light sources for uncaging can be categorised into general- and specific-purpose lamps/lasers. ${ }^{90}$

General-purpose light sources like $\mathrm{Hg}, \mathrm{Xe}$ and metal halide lamps, are used for imaging as well as for the photocleavage reaction. These lamps exhibit broad emission spectra between 250 - $700 \mathrm{~nm}$ (figure 3.1) which overlap with absorption of PPGs as well as commonly-used fluorophores, and therefore provide the advantage of two applications in one device by switching between excitation filters. On the other hand, simultaneous imaging and uncaging as well as fine-tuning of the intensity for photocleavage is not possible. $\left.{ }^{90}\right]$ 


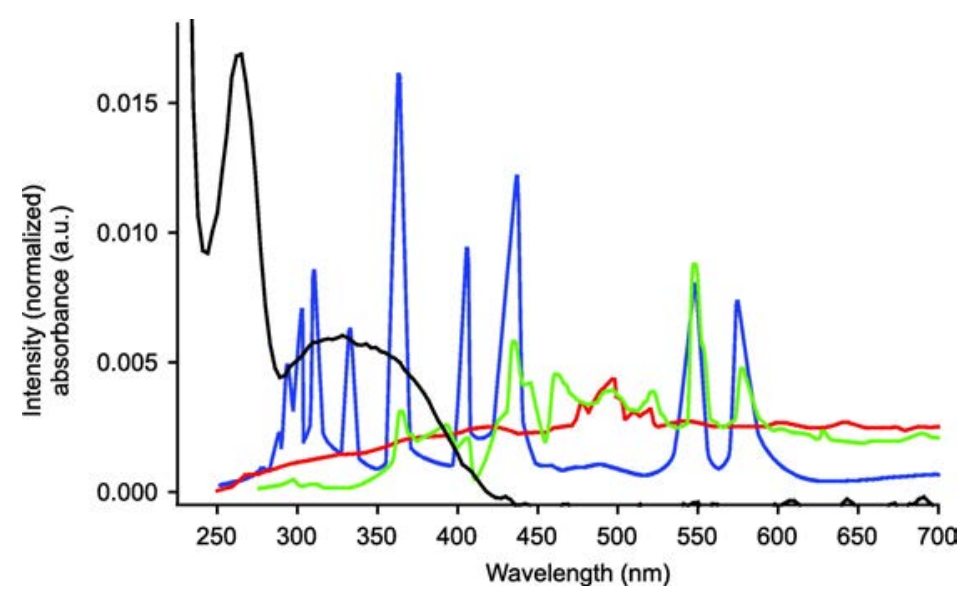

Figure 3.1: Emission spectra of general-purpose light sources: Hg lamp (blue curve), Xe lamp (red curve) and metal halide lamp (green curve). For comparison, absorption spectra of a commonly used PPG (6-nitroveratryl, NV) is shown (black curve). Figure used from LEE et al. 90 Reprinted with permission from reference [90]. Copyright (c) 2009 American Chemical Society.

$\mathrm{Hg}$ lamps provide most power in the UV region including spectral lines at 312, 334, and $365 \mathrm{~nm}$ (figure 3.1. blue curve) and minimal excitation in the infrared region $(\lambda>700 \mathrm{~nm})$ which results in less heating of the sample. Xe lamps exhibit a homogeneously distributed excitation spectrum from near UV to infrared light (figure 3.1. red curve). Without intensity differences between the corresponding channels, a broad range of fluorophores can be excited and photocleavage reactions can be performed efficiently. However, due to the significant emission in the infrared region, heating of the sample needs to be prevented by blocking filters. Metal halide lamps are more and more used for fluorescence imaging but exhibit only low intensities for $\lambda<350 \mathrm{~nm}$ (figure 3.1. green curve). Due to the fact that most PPGs exhibit the highest absorption between $320 \mathrm{~nm}$ and $370 \mathrm{~nm}$ (e.g. 6-nitroveratryl (NV), figure 3.1. black curve), these lamps are not suitable for most photocleavage reactions. Only when coupled with a UV laser, metal halide lamps can be applied for separately imaging and uncaging. 90]

Specific-purpose light sources include UV lasers like $\mathrm{N}_{2}$ gas and solid-state lasers and are used for uncaging alone. These systems coupled with a separate imaging pathway exhibit the following benefits: The light intensity for uncaging can be specifically adjusted with higher spatial and/or temporal resolution. With suitable filters and a dichroic design, simultaneous imaging and irradiation for photocleavage is applicable due to the substantial blue-shift from the imaging wavelengths. Since the optical pathway for UV irradiation is fixed, no switching of excitation filters is necessary and the intensity can be independently controlled by the applied laser power. For even more precision, pulsed UV lasers can be used. In summary, specific-purpose light sources provide the great advantage of simultaneous imaging and uncaging of fast processes in one device. 90

A third category of instruments for uncaging comprises ultrafast IR lasers which can be 
used for two-photon uncaging (chapter 3.4). With this technique, suitable PPGs can be excited with $\sim 2 \lambda_{\max }$, but this also requires laser light sources, such as a pulsed titanium-sapphire laser, which emit light in the range of $650-1100 \mathrm{~nm}$. 90 [99]

\subsection{Most Frequently Used Photocleavable Protecting Groups}

\subsection{1 o-Nitrobenzyl Groups}

Besides the considerable number of disadvantages, o-nitrobenzyl (NB) compounds, especially the dimethoxy derivative (4,5-dimethoxy-2-nitrobenzyl or 6-nitroveratryl, NV), represent the most frequently used PPGs (scheme 3.2). 91 92] The o-nitrobenzyl group was firstly described as a PPG by SCHOFIELD et al. ${ }^{[00]}$ in 1966, followed by the o-nitrobenzyloxycarbonyl (Nboc) and 6nitroveratryloxycarbonyl (Nvoc) derivatives attached to amino acids described by WoODWARD et al.101] in 1970.9192] The simple structure and various substitution possibilities provide straightforward synthetic procedures as well as practical attachment to a variety of functional groups of the target compounds. In addition, a wide range of substitution patterns for the NB moiety provides a crucial tool to adjust the absorption spectrum, quantum yield and the stability of the photocleaved bond. Without further substitution, NB-caged compounds exhibit a maximal absorption at $\lambda_{\max }=320 \mathrm{~nm}$ which is not suitable for in vivo applications. However, by substitution of the aromatic and/or benzylic positions, a significant bathochromic (red) shift of the absorption maximum can be achieved. Especially, introduction of a moderate electrondonating group (e.g. OMe) at the meta- and para-positions, leading to the NV derivatives, was shown to result in cleavage at considerably longer wavelength $\left(\lambda_{\max }=345 \mathrm{~nm}\right)$. On the other hand, benzylic substitutions can result in a significant hypsochromic (blue) shift but mainly have an impact on the quantum yield and prevent formation of the toxic nitrosoaldehyde side product. 9192 .<smiles>O=[N+]([O-])c1ccccc1CP</smiles>

NB<smiles>[R]C(=O)OCc1ccccc1[N+](=O)[O-]</smiles>

$\mathrm{Nboc}$<smiles>COc1cc(CP)c([N+](=O)[O-])cc1OC</smiles>

NV<smiles>COc1cc(COC=O)c([N+](=O)[O-])cc1OC</smiles>

Nvoc<smiles>COc1cc(C(=O)O)c([N+](=O)[O-])cc1OC</smiles>

$\alpha-\mathrm{CNV}$

Scheme 3.2: Structures of the o-nitrobenzyl (NB) moiety and its derivatives used as PPGs: o-nitrobenzyloxycarbonyl (Nboc), 6-nitroveratryl (NV), 6-nitroveratryloxycarbonyl (Nvoc), and $\alpha$-carboxy-6-nitroveratryl ( $\alpha$-CNV) derivatives. The corresponding target molecule is indicated as residue $\mathrm{R}$.

In addition, this side product is even more problematic as it reacts with amines to form stable imine derivatives and degradation products which act as an internal filter. The former can be prevented by addition of semicarbazide hydrochloride as a scavenger. ${ }^{92}$. Substitution with 
a methyl group was found to increase the quantum yield and led to the less toxic and less problematic acetophenone side product. 9102 102] Nevertheless, it needs to be considered that benzylic substitutions create a chiral center and could complicate caging of chiral molecules. ${ }^{92}$. The water solubility needed for biological applications can be increased by substitution with $\mathrm{CO}_{2} \mathrm{H}$ moieties. For this purpose, the $\alpha$-carboxy-6-nitroveratryl $(\alpha$-CNV) derivative was developed which, besides better solubility in aqueous media, exhibits also a red shift of maximal absorption wavelength $\left(\lambda_{\max }=356 \mathrm{~nm}\right)$. 92 103] The third position for adjustment of the photocleavage properties is the linkage between the PPG and the target molecule. Most target molecules can be attached directly at the benzylic site, but especially for alcohols and amines, the attachment as a carbonic acid derivative (e.g. Nvoc) is widely used. This is based on the straightforward synthesis by the reaction of the alcohol or amine with the PPG chloroformate. However, the oxycarbonyl linkage was shown to decrease the rate of photolysis significantly, and thus, is unsuitable for rapid release applications. In summary, although a variety of different substitution patterns were tested for o-nitrobenzyl derivatives, often contradictory results led to no clear tendency for the development of new PPGs, due to different excitation wavelengths or different leaving groups used. 92 .

\subsubsection{Coumarin-4-ylmethyl Groups}

The second most frequently used PPGs are coumarin-4-ylmethyl (CM) derivatives, which have the advantages of relatively simple synthesis, although not as straightforward as for NB derivatives (scheme 3.3). These PPGs exhibit high absorption coefficients even at longer wavelength, fast photocleavage rates, and fluorescent properties for easy reaction monitoring. In addition, CM provides a variety of substitution possibilities to obtain a library of derivatives with a wide range of maximal absorption wavelengths $\left(\lambda_{\max }=310-490 \mathrm{~nm}\right) .91 .92$

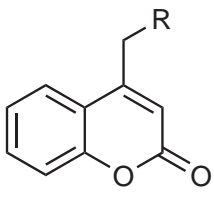

CM

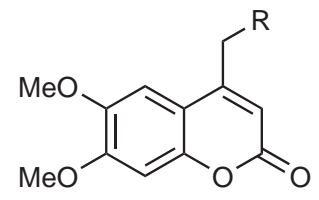

DMCM<smiles>[2H]Cc1cc(=O)oc2cc(NCC)ccc12</smiles>

DEACM<smiles>[R]Cc1c(/C=C/C(=O)N[C@@H](CC(=O)O)C(=O)O)c(=O)oc2cc(NCC)ccc12</smiles>

DEACM450

Scheme 3.3: Structures of the coumarin-4-ylmethyl (CM) moiety and its derivatives used as PPGs: 6,7-dimethoxy(coumarin-4-yl)methyl (DMCM), (7-(diethylamino)coumarin-4-yl)methyl (DEACM) derivatives, and (E)-2-(3-(7-Diethylamino)-4-(hydroxymethyl)-2-oxo-2H-chromen-3$\mathrm{yl}$ )acrylamido)succinate (DEACM450). The corresponding target molecule is indicated as residue $\mathrm{R}$.

Without further substitution, CM-caged compounds exhibit a maximal absorption at $314 \mathrm{~nm}$ which is not suitable for in vivo applications. The first generation of CM PPGs, such as the 6,7-dimethoxy(coumarin-4-yl)methyl (DMCM) derivative (scheme 3.3), exhibits only low water solubility but already applicable absorption properties $\left(\lambda_{\max }=344 \mathrm{~nm}\right)$. For the second 
generation, substitution with amino groups in position 7 were introduced to obtain absorption maxima between $350 \mathrm{~nm}$ and $400 \mathrm{~nm}$, e.g. the (7-(diethylamino)coumarin-4-yl)methyl (DEACM) derivative with $\lambda_{\max }=396 \mathrm{~nm}$ (scheme 3.3). 92 104] Further substitutions at position 3 were identified to be crucial for absorption maxima adjustment and different substituents were tested by ELLIS-DAVIS et al.105 Especially, substitution with an acrylamido succinic acid moiety led to a strong red shift of the absorption maximum $\left(\lambda_{\max }=450 \mathrm{~nm}\right)$ and significantly better water solubility (DEACM450, scheme 3.3). Attached to the glutamic acid side chain, the DEACM450 compound exhibits very fast and clean photolysis with high quantum yields. In addition, it is suitable for two-photon excitation at $900 \mathrm{~nm}$. 106. The two-photon uncaging technique for photolysis provides several advantages and is discussed in the following chapter. Furthermore, the DEACM450 moiety exhibits only low two-photon-excitation at $\sim 720 \mathrm{~nm}$ in which region most other PPGs can be excited. [106] Therefore, application of DEACM450 is possible in combination with a second PPG for wavelength-selective photolysis (chapter 3.5). On the other hand, a nine step synthesis is needed for DEACM450-caged glutamic acid, 106] whereas an o-nitrobenzyl-caged glutamic acid, e.g. caged with the 3-(4,5-dimethox-2-nitrophenyl)-2-butyl (DMNPB) derivative, is obtained in five steps. 107. Hence, a cost-benefit analysis for the corresponding application needs to be considered.

\subsection{Two-Photon Uncaging}

Two-photon uncaging is an alternative to the "normal" single-photon excitation of PPGs and the irradiation is performed at $\sim 2 \lambda_{\max }$. For example, o-nitrobenzyl derivatives with $\lambda_{\max }=365 \mathrm{~nm}$ can be excited with light of $730 \mathrm{~nm}$. Not all PPGs are suitable for this method and the nonlinear optical effect needs high light intensities. However, this method has several advantages: A precise correlation between image pixel and excitation spot of the laser provides three-dimensional resolution of the uncaging area. Due to the focussed laser beam, distinct small areas or volumes (up to $1 \mathrm{fL}$ ) of the sample can be addressed for photolysis (figure $3.4 \mathrm{~b}$ ). In addition, a deeper penetration of tissue is obtained and less photodamage occurs by the application of infrared light. 90 99.

Analogous to the product of extinction coefficient and quantum yield $(\varepsilon \Phi)$ for single-photon uncaging, the two-photon cross-section $\delta_{\mathrm{a}}$ and action cross-section $\delta_{\mathrm{u}}$ in GOEPPERT-MAYER (GM) units are used to quantify the efficiency of the PPG's two-photon effect. For biological applications, $\delta_{\mathrm{u}}>0.1 \mathrm{GM}$ was suggested to be necessary. ${ }^{[99]}$ However, although the NV group exhibits only low two-photon action cross-section $\left(\delta_{\mathrm{u}}=0.025-0.035 \mathrm{GM}\right)$, it was nevertheless applied for two-photon uncaging. ${ }^{99}$ As already mentioned, the most promising DEACM450 PPG exhibits very good two-photon properties with a two-photon-uncaging cross-section of $0.5 \mathrm{GM}$ at $900 \mathrm{~nm}$. 106] The two-photon uncaging method provides further application possibilities for wavelength-selective photocleavage, due to the fact that a wider range of irradiation wavelengths of $700-900 \mathrm{~nm}$ can be applied. For single-photon uncaging selectivity 
a

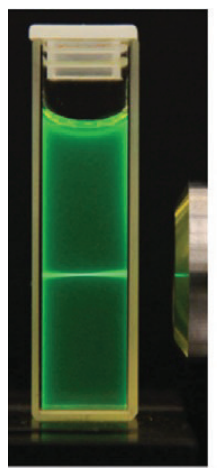

b

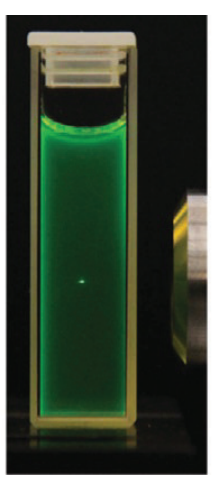

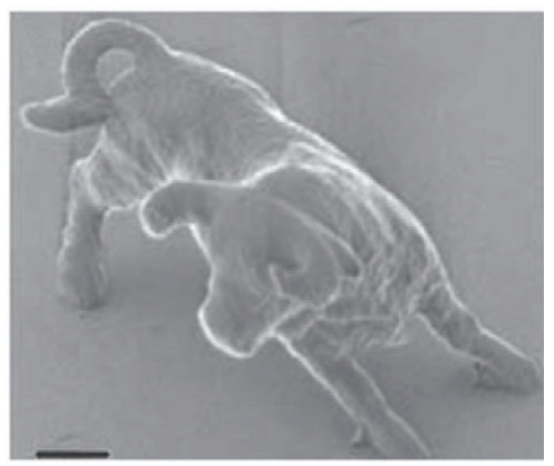

Figure 3.4: Comparison of single- and two-photon excitation by the example of a fluorescein solution. (a) With single-photon irradiation $(365 \mathrm{~nm}$ ) the excitation results in a double cone. (b) With two-photon irradiation $(730 \mathrm{~nm})$ a three-dimensionally resolved excitation spot can be obtained. Figures adapted from BRIEKE et al.99] (c) 2012 Wiley-VCH Verlag GmbH \& Co. KGaA, Weinheim. (c) Example for application of two-photon excitation: Light-induced polymerisation of a tiny bull's figurine (scale bar $2 \mu \mathrm{m}$ ). Figure used from KAWATA et al. 108 Reprinted by permission from Macmillan Publishers Ltd: Nature, reference [108], copyright $(C$ 2001.

of two PPGs in the relatively small range of $350-450 \mathrm{~nm}$ is more complicated to achieve. The strategy of wavelength-selective photocleavage is discussed in the following chapter.

\subsection{Wavelength-Selective Photocleavage}

With the method of wavelength-selective photocleavage, it is possible to address two or more processes separately (scheme 3.5). By caging two or more functionalities with PPGs exhibiting e.g. different absorption maxima $\left(\lambda_{\max }\right)$, these functionalities can be selectively activated upon irradiation with the corresponding wavelength. 91] In 2000, the strategy was firstly described by BOCHET ${ }^{[09]}$ and applied to the selective uncaging of a mixture of Nvoc-caged amine and 3,5-dimethoxybenzyl-caged carboxylic acid. As already mentioned in chapter 3.3.1, the Nvoc PPG could be cleaved by irradiation at $\lambda=350 \mathrm{~nm}$, whereas the 3,5-dimethoxybenzyl group needed irradiation at $\lambda=254 \mathrm{~nm}$. 109$]$ Not all PPGs can be applied for wavelength-selective uncaging, as several requirements were identified: The absorption spectrum should exhibit a narrow absorption maximum with high quantum yields and low absorption outside this region. In addition, no intra- and/or intermolecular energy transfer should be observed for the corresponding PPG. The different molecule classes of PPGs provide a selection of absorption maxima with the result to combine PPGs with large differences of $\lambda_{\max }$. For example, the DEACM450 PPG could be combined with NV or Nvoc derivatives to obtain a high selectivity and high availability for different functionalities. ${ }^{91}$

However, most of the PPGs cleavable with light of longer wavelength in the visible region also absorb light in the UV region. Meaning, uncaging of the UV-cleavable PPG will also partially cleave the putatively selective PPG. Consequently, selective uncaging is only possible 


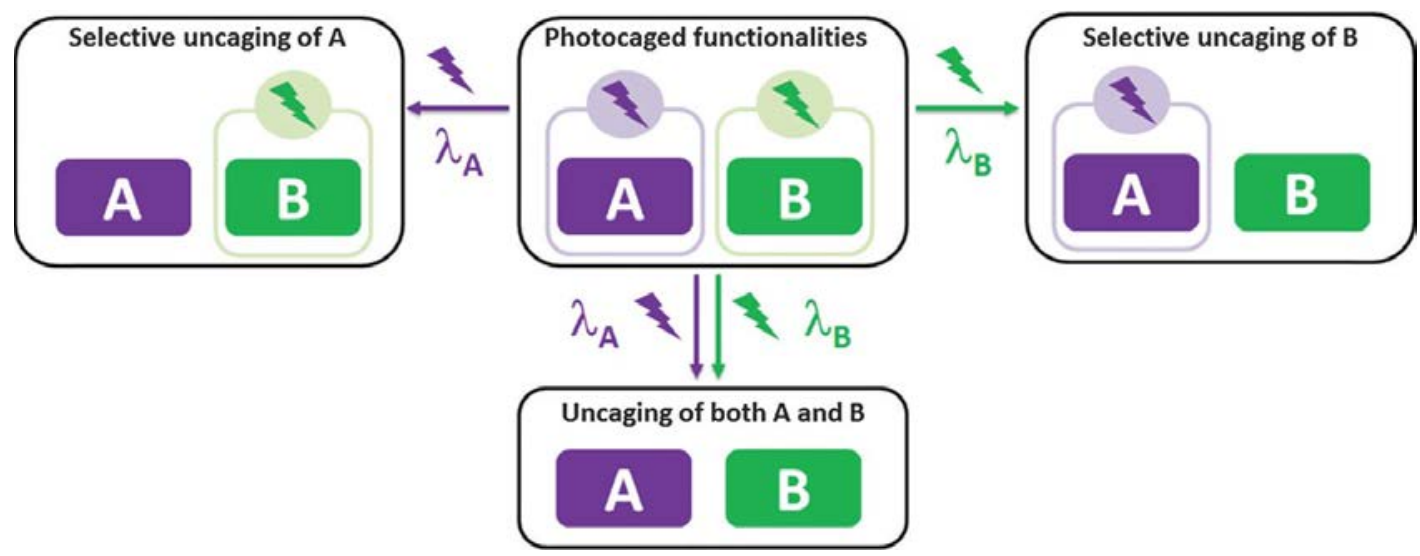

Scheme 3.5: Principle of wavelength-selective uncaging: (left) Irradiation with wavelength $\lambda_{\mathrm{A}}$ will selectively uncage functionality $A$ (purple) while functionality $B$ (green) remains caged. (right) Only irradiation with wavelength $\lambda_{\mathrm{B}}$ will selectively uncage functionality $\mathrm{B}$. (middle) Irradiation with both wavelengths will also uncage both functionalities. Scheme used from HANSEN et al. 91 ] Reproduced from reference [91] with permission of The Royal Society of Chemistry.

in a certain sequence, such as irradiation at longer wavelength followed by irradiation at shorter wavelength. 91 ]

An interesting example for wavelength-selective uncaging is the application for SPPS.110 As described in chapter 4.2, synthesis of peptides by SPPS requires a variety of orthogonal protecting groups which need to be selectively cleaved under mild conditions. Application of PPGs for this purpose provides the advantage of orthogonality and less interference to other reaction conditions. In 2003, the first attempt led to the successful synthesis of a pentapeptide by SPPS including PPGs, a photocleavable linker to the solid-support, and without a single chemical deprotection step. 91 110] For temporal $N$-terminal protection of the amino acids, Nvoc was used as PPG and cleaved by irradiation at $360 \mathrm{~nm}$. The sample was irradiated for $20 \mathrm{~min}$ and formation of the aldehyde side product was prevented by addition of semicarbazide hydrochloride. The photocleavable linker to the solid-support based on a 1,2-dihydroxy-2,4,4trimethylpentan-3-one moiety which was cleaved at the end of the synthesis by irradiation at $305 \mathrm{~nm}$. This procedure provides access to the synthesis of peptides containing sensitive side chains or side chain protecting groups at neutral $\mathrm{pH}$. 110]

More recently, PPGs were applied for solution-phase peptide synthesis to avoid usage of highly reactive species (e.g. TFA, piperdine) as it would be needed for example for in vivo synthesis on microchips or automated experiments in space. ${ }^{111}$ In this concept, for $C$-terminal protection of the amino acid, the 5,7-dinitroindolinyl (Dni) moiety was used (scheme 3.6). As $N$-acylated derivative, this PPG was shown to react with a nucleophile by an acyl transfer upon irradiation at $\lambda>385 \mathrm{~nm}$. The amino group of the amino acids was protected by the 3,5-dimethoxy- $\alpha, \alpha$-dimethylbenzyloxycarbonyl ( $\mathrm{Ddz}$ ) compound which was cleaved by irradiation at $\lambda<350 \mathrm{~nm}$ (scheme 3.6). By the application of an LED-based reactor for 


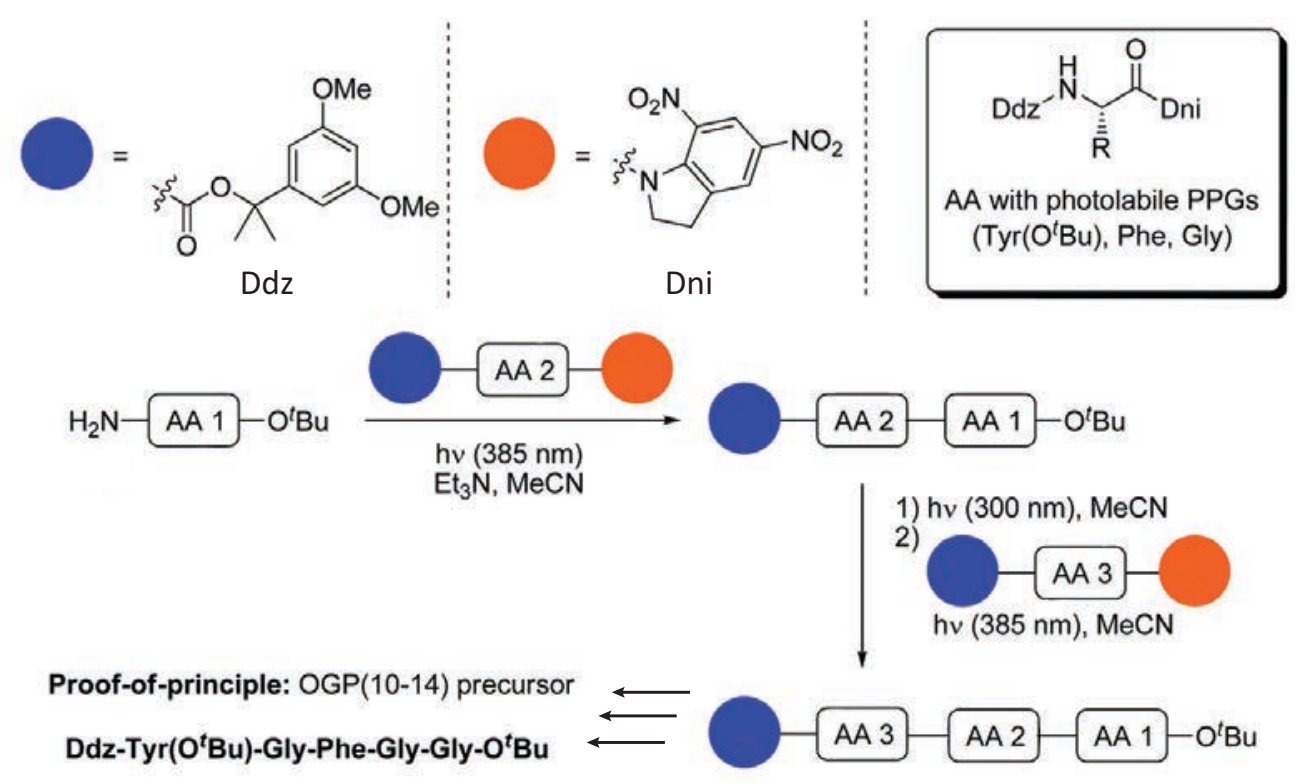

Scheme 3.6: Example of solution-phase peptide synthesis including wavelength-selective PPGs: 3,5-dimethoxy- $\alpha, \alpha$-dimethylbenzyloxycarbonyl (Ddz) cleavable by irradiation at $\lambda=385 \mathrm{~nm}$ and 5,7-dinitroindolinyl (Dni) cleavable at $\lambda=300 \mathrm{~nm}$. Scheme modified from HANSEN et al. [91] Adapted from reference [91] with permission of The Royal Society of Chemistry.

uncaging of Dni at $385 \mathrm{~nm}$, and sequential usage of a RAYONET reactor for uncaging of Ddz at $300 \mathrm{~nm}$, a pentapeptide was successfully synthesized without additional reagents. However, further optimisations of this method are needed since purification of intermediates and elaborate building block synthesis are still required. 91 111.

\subsection{Biological Applications of PPGs in Neuroscience}

In neuroscience, PPGs as well as wavelength-selective uncaging were already successfully applied several times. With caged excitatory (e.g. glutamic acid, Glu) and inhibitory (e.g. $\gamma$-amino butyric acid, GABA) neurotransmitters, neurons and even single synapses were investigated. In this context, wavelength-selective uncaging of the corresponding neurotransmitter in combination with two-photon uncaging provides high spatio-temporal control of the release, excitatory or inhibitory signalling, and distribution. ${ }^{91]}$ One recent example is the combination of DEACM450-caged Glu and 4-carboxymethoxy-7-nitroindolinyl (CDNI)-caged GABA. 112] As already described in chapter 3.3.2 and 3.4. DEACM450-caged Glu exhibits an absorption maximum at $\lambda_{\max }=450 \mathrm{~nm}$ and a two-photon-uncaging cross-section of $0.5 \mathrm{GM}$ at $900 \mathrm{~nm}$. On the other hand, the CDNI moiety showed an absorption maximum at $\lambda_{\max }=330 \mathrm{~nm}$ and a two-photon-uncaging cross section of $0.06 \mathrm{GM}$ at $720 \mathrm{~nm}$. ${ }^{106}$ When both compounds were simultaneously applied to a brain slice, it was possible to control the membrane potential in the excitatory as well as in the inhibitory direction by wavelength-selective two-photon uncaging at $720 \mathrm{~nm}$ and $900 \mathrm{~nm}$, respectively. This was also the case for DEACM450-caged GABA and 
CDNI-caged Glu. 112

Another successful example for caging in neurobiology was initially developed by NEHER \& ZUCKER ${ }^{[13}$ 114] in 1993. Today, the application of caged $\mathrm{Ca}^{2+}$ is well-established for studying exocytosis in vivo. By applying a UV flash, this method mimics the jump of $\mathrm{Ca}^{2+}$ concentration as it would occur upon $\mathrm{Ca}^{2+}$ influx into a nerve terminal. 115. Since cations such as $\mathrm{Ca}^{2+}$ cannot be covalently caged, a variety of photocleavable chelators with high affinity towards $\mathrm{Ca}^{2+}$ were developed. ${ }^{116}$

In 1988, one of the first caged $\mathrm{Ca}^{2+}$ compounds was 1-(2-nitro-4,5-dimethoxyphenyl)- $N, N, N^{\prime}, N^{\prime}$ tetrakis[(oxycarbonyl)methyl]-1,2-ethandiamine (DM-nitrophen) which is based on the NV PPG (scheme 3.7). 117. Soon after, it became commercially available, and consequently, it was also easily accessible for biologists. Upon irradiation, the backbone and coordination sphere of the chelator is cleaved which results in a dramatic decrease of $\mathrm{Ca}^{2+}$ binding affinity. Before uncaging, high affinity for $\mathrm{Ca}^{2+}$ was detected $\left(K_{d}=5 \mathrm{nM}\right)$, whereas afterwards, the products of photocleavage exhibited only low affinity (in average $K_{\mathrm{d}}=3 \mathrm{mM}$ ). In addition, DM-nitrophen provides a relatively high quantum yield $(\Phi=0.18)$ at $\lambda=350 \mathrm{~nm}$ and high release rates of $\mathrm{Ca}^{2+}\left(38000 \mathrm{~s}^{-1}\right)$. However, the structure of this PPG is based on ethylenediaminetetraacetic acid (EDTA) and exhibits also non-negligible affinity towards $\mathrm{Mg}^{2+}$ and protons. Surprisingly, this disadvantage was found to be unimportant for most physiological applications. ${ }^{[116]}$ With DM-nitrophen and other caged $\mathrm{Ca}^{2+}$ compounds, the basic questions of $\mathrm{Ca}^{2+}$ influence on neurotransmitter release could be addressed.116] Nowadays, nitrophenyl-ethylene glycol-bis(2aminoethylether- $N, N, N^{\prime}, N^{\prime}$-tetraacetic acid (NP-EGTA, scheme 3.7) is used to investigate the mechanisms of membrane fusion machinery (e.g. VERHAGE et al. $\left.{ }^{[18]}\right)$.<smiles>COc1cc(C(CN(CC(=O)O)CC(=O)O)N(CC(=O)O)CC(=O)O)c([N+](=O)[O-])cc1OC</smiles>

DM-nitrophen<smiles>O=C([O-])CN(CCOCCOCC(c1ccccc1[N+](=O)[O-])N(CC(=O)[O-])CC(=O)[O-])CC(=O)O</smiles>

NP-EGTA

Scheme 3.7: Examples of photocleavable $\mathrm{Ca}^{2+}$ chelators: 1-(2-nitro-4,5-dimethoxyphenyl)$N, N, N^{\prime}, N^{\prime}$-tetrakis[(oxycarbonyl)methyl]-1,2-ethandiamine (DM-nitrophen) and nitrophenylethylene glycol-bis(2-aminoethylether)- $N, N, N^{\prime}, N^{\prime}$-tetraacetic acid (NP-EGTA). 


\section{Synthesis and Analyses of Adamantyl-Modified Synaptobrevin Analogues}

The development of a suitable inhibitor for the SNARE disassembly reaction requires, first of all, a detailed analysis of the interaction between $\alpha$-SNAP/NSF and the SNARE complex (chapter 4.1). As a scaffold for the inhibitor, the SNARE protein Synaptobrevin-2 was chosen due to its simple domain structure in comparison to Syntaxin-1A or SNAP-25. Specific amino acid side chains of Synaptobrevin-2 SNARE motif had to be identified, which are essential for the interaction with $\alpha$-SNAP. The modification of these positions should influence the binding properties, but on the other hand, should not influence the SNARE complex assembly with the natural counterparts, Syntaxin-1A and SNAP-25. The design of Synaptobrevin-2 analogues was developed previously in our group by Dr. A. Groschner. 119.

After successful synthesis of the modified Synaptobrevin-2 derivatives by SPPS (chapter 4.3), ternary SNARE complexes were formed with their natural counterparts (Syntaxin-1A and SNAP-25) and reconstituted into small unilamellar vesicles (SUVs, chapter 4.5.1). The assembly properties were investigated in detail using stabilised acceptor complexes $(\Delta \mathrm{N}$ complex, chapter 4.5.2). The disassembly reaction of modified ternary SNARE complexes on SUVs was monitored (chapter 4.5.3). In addition, Complexin binding properties to SNARE complexes containing modified Synaptobrevin-2 analogues were investigated (chapter 4.6). The main spectroscopic method used was fluorescence anisotropy which is described in chapter 4.4 .

\subsection{The Interaction Between $\alpha$-SNAP and SNARE Complex}

The binding stoichiometry of $\alpha$-SNAP to the SNARE complex is still under discussion, and was recently found to be flexible depending on the SNARE complex composition. 50 [5] In 2003, MARZ et al. identified the $\alpha$-SNAP residues which are interacting with the SNARE complex by site-directed mutagenesis and assumed a $3 / 1$ stoichiometry of the $\alpha$-SNAP/SNARE complex. [64] Especially charged residues at the $N$-terminal concave surface of $\alpha$-SNAP were identified to influence binding affinity to the SNARE complex. Mutation of positively charged 
residues to alanines resulted in a decreased binding to the SNARE complex and a less efficient disassembly reaction, whereas mutation of negatively charged residues to alanines increases binding to the SNARE complex. Consequently, the binding between $\alpha$-SNAP and SNARE complex is based on electrostatic interactions of positively charged lysine residues of $\alpha$-SNAP and negatively charged aspartic and glutamic acid residues of SNARE complex. 64

MARZ et al. were able to identify six distinct interaction sites within the $\alpha$-SNAP sequence: $\mathrm{K} 56, \mathrm{~K} 93, \mathrm{~K} 94, \mathrm{~K} 122, \mathrm{~K} 163$, and K167. These residues are localised like a diagonal tape across the concave site of $\alpha$-SNAP (figure 4.1 a). On the surface of the SNARE complex complementary binding partners were determined. Likewise diagonally oriented, negatively charged residues define three possible binding sites for $\alpha$-SNAP (figure $4.1 \mathrm{~b}$ ). Especially within the Synaptobrevin-2 SNARE motif sequence, residues D51, E55, and D65 were identified. These residues are close to the hydrophilic zero layer R56 (figure $4.1 \mathrm{c}$, left) and cover binding sites for two $\alpha$-SNAPs. ${ }^{64]}$ Considering their orientation, charge, and close proximity to the zero layer, the additional residues Q58 and E62 were also addressed as possible interaction sites (figure 4.1 c, right). 119$]$

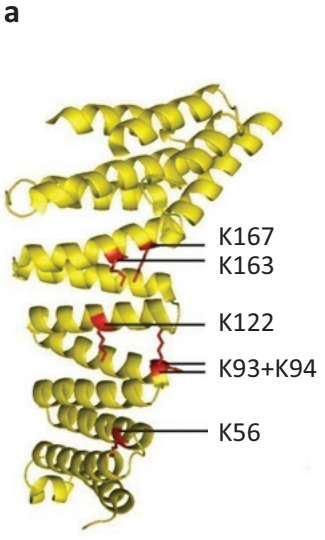

$\alpha-S N A P$

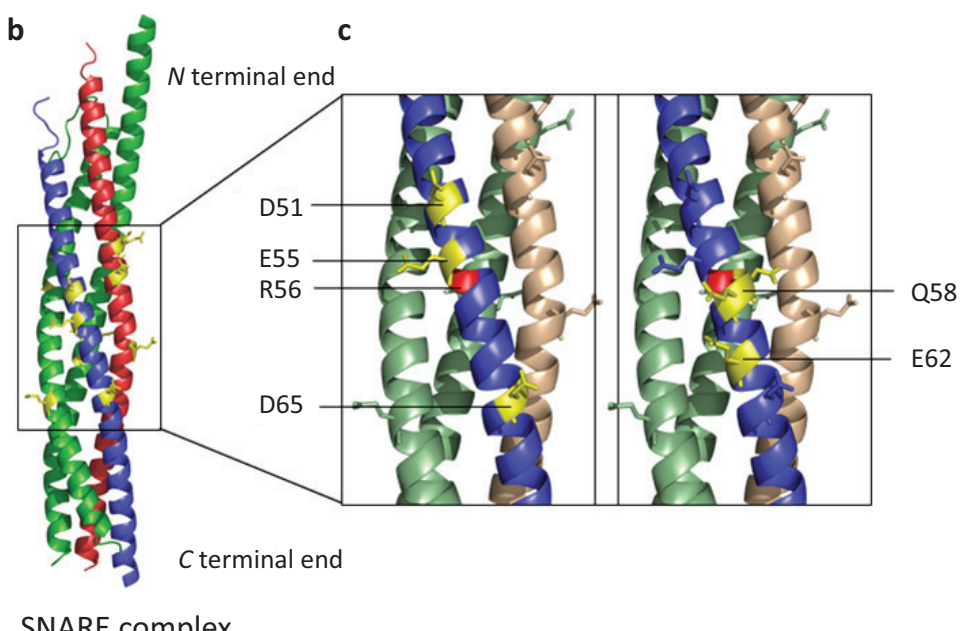

SNARE complex

Figure 4.1: (a) Structure of $\alpha$-SNAP (pdb code 1qqe) with positively charged lysine residues at the site of interaction highlighted in red. (b) Conserved negatively charged residues of SNARE complex highlighted in yellow. Neuronal SNARE complex (pdb code 1sfc) consisting of Synaptobrevin-2 (blue), Syntaxin-1A (red), and SNAP-25 (green). (c) Detailed view on possible interaction sites with $\alpha$-SNAP on the SNARE complex surface within Synaptobrevin-2 SNARE motif sequence: D51, E55, Q58, E62, and D65. Hydrophilic zero layer R56 is highlighted in red. Figure modified from A. GROSCHNER. 64 119.

With respect to the study by MARZ et al. ${ }^{64}$ modified Synaptobrevin-2 analogues were designed. ${ }^{[119]}$ Besides preventing the $\alpha$-SNAP binding to the SNARE complex surface, these derivatives should be able to form the ternary SNARE complex with their natural counterparts Syntaxin-1A and SNAP-25.

The modified Synaptobrevin-2 derivatives consisting of the complete SNARE motif sequence (aa 28 - 84, figure 4.2 a) were synthesised by solid-phase peptide synthesis (SPPS, chapter 4.2 
and 8.4). The transmembrane domain, the linker region, as well as the proline-rich $N$-terminal domain are not essential for ternary SNARE complex formation. 120 However, amino acid residues 28 - 32 turn out to be critical for complex formation, due to the fact that no complex formation could be observed for Synaptobrevin-2 which lacked these residues. 119 .

a

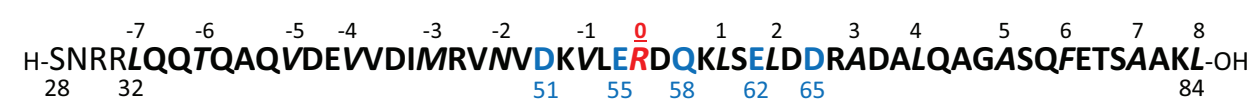

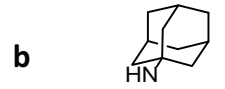

Fmoc:

Figure 4.2: (a) Synaptobrevin-2 SNARE motif sequence (aa 28 - 84) with modified positions highlighted in blue, zero layer R56 in red, hydrophobic layers -7 to +8 in italic, and SNARE motif contributing to SNARE complex formation in bold. (b) Unnatural amino acid building block Fmoc-Asn(Ad)-OH (N(Ad), 4). 119]

Firstly, the three amino acid residues D51, E55, and D65, suggested by MARZ et al. to be the binding site for $\alpha$-SNAP, were substituted by lysines. These substitutions should change the charge on the surface and thereby generate a repulsive interaction with $\alpha$-SNAP. Unfortunately, no ternary complex formation was observed for this derivative (Syb-2 (28 - 84) D51K, E55K, D65K). 119.

For further modification, an unnatural amino acid building block 4 with a sterically demanding side chain (1-adamantane) was designed (figure 4.2 b). ${ }^{119}$ This modification should inhibit or weaken $\alpha$-SNAP binding to the SNARE complex surface by sterical hindrance. The building block was developed for application during SPPS following the standard Fmoc protocol. It is based on the amino acid asparagine (Asn, $\mathrm{N}$ ) with its side chain modified by an 1-adamantyl residue. This building block was introduced at each possible interaction site within the sequence. In addition, derivatives with two or three modifications in one sequence were synthesised (chapter 4.3) and analysed regarding their SNARE complex disassembly properties by fluorescence anisotropy (chapter 4.5).

\subsection{Solid-Phase Peptide Synthesis (SPPS)}

The synthesis of adamantyl-modified Synaptobrevin-2 analogues was conducted by SPPS developed by R. B. MERRIFIELD. The advantage of this synthesis method is the covalent attachment of the solid support (resin) to the growing peptide sequence. Thereby, during the synthesis, the peptide sequence is insoluble in the corresponding solvent, but reagents and by-products can be easily separated by washing and filtration. No further purification step is needed after the coupling of each amino acid. This procedure essentially increases the yields of peptide synthesis. 121 122.

For the stepwise peptide synthesis from $C$ - to $N$-terminal end, an orthogonal strategy of protecting groups is necessary. Therefore, the $N$-terminal end and, if necessary, amino acid side 


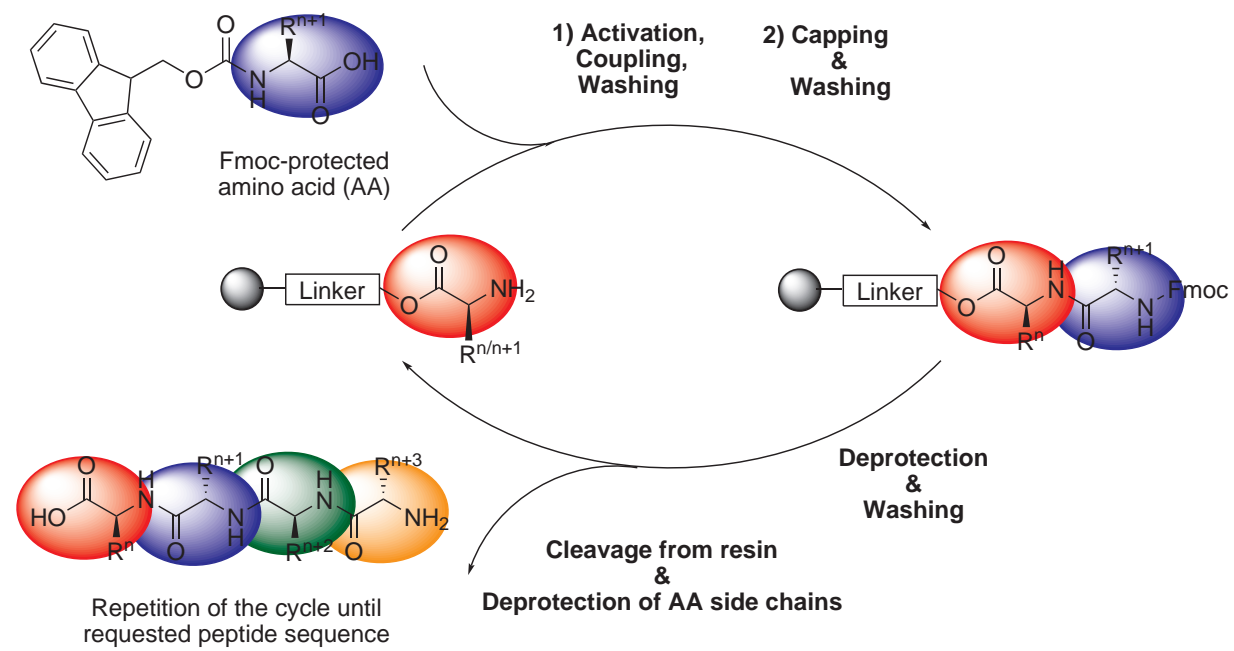

Scheme 4.3: Synthesis cycle of solid-phase peptide synthesis (SPPS) using standard Fmoc protocol. Starting with activation of carboxy function, coupling reaction, followed by capping reaction to deactivate the uncoupled amine functionalities, and ultimately, deprotection of amine functionalities. Upon accomplishment of the desired peptide sequence, cleavage from the solid support and complete deprotection of the amino acid side chains were conducted.

chains, have to be protected with orthogonal protecting groups for specific deprotection. The standard Fmoc protocol is most commonly used (scheme 4.3): The $\alpha$-amine group is protected by 9-fluorenylmethoxycarbonyl (Fmoc) group which can be removed by bases. On the other hand, the amino acid side chains are protected with protecting groups which can be cleaved by acids. The advantage of this strategy is that, during synthesis, the Fmoc protecting group can be specifically removed by piperidine (20\% in NMP), whereas all amino acid side chains remain protected, avoiding possible side reactions. For all steps, $N$-methyl-2-pyrrolidinone (NMP) or dimethylformamide (DMF) are utilised as solvent. ${ }^{123+-125}$.

The standard Fmoc protocol has the advantage to be easily automated due to non-corrosive substances used in the synthetic procedure. In addition, Fmoc deprotection releases dibenzofulvene which exhibits high UV absorption and can be used as an indicator for successful deprotection and coupling reaction. ${ }^{125}$ ]

After deprotection of the $\alpha$-amine function, coupling of the next amino acid of the peptide sequence is performed. For peptide bond formation, it is necessary to first activate the $\alpha$ carboxyl function of the incoming amino acid. A variety of activating reagents are commercially available for the coupling step and for each amino acid building block it has to be considered which one will lead to the best yields. The mixture of 2 -( $1 H$-benzotriazole-1-yl $)-1,1,3,3,-$ tetramethyluronium-hexafluorophosphate (HBTU) and 1-hydroxybenzotriazole ( $\mathrm{HOBt})$ with diisopropylethylamine (DIPEA) as base is often used for natural amino acids. HOBt is utilised to accelerate the reaction and to prevent racemisation. The activation reaction with HBTU proceeds via formation of acyluronium intermediate which reacts with deprotonated $\mathrm{HOBt}$ to 1-hydroxybenzotriazol ester. The subsequent acylation of the amine leads to regeneration of HOBt. 124,126 
A more efficient activating reagent is the analogue 2-(1H-7-azabenzotriazol-1-yl)-1,1,3,3tetramethyluronium-hexafluorophosphate (HATU) which is used in combination with the corresponding additive 1-hydroxy-7-azabenzotriazole (HOAt). The reason for the higher efficiency is the formation of the active ester with HATU, an aza-analogue structure in which the amine as the coupling partner is favourably oriented. 126$]$

However, HOBt and HOAt, both have the disadvantage to be classified as explosives, which leads to transportation difficulties and consequently restricts commercial availability. As an alternative, ethyl 2-cyano-2-(hydroxyimino)acetate (Oxyma) used in combination with $\mathrm{N}, \mathrm{N}$ diisopropylcarbodiimide (DIC) showed clear advantages over $\mathrm{HOBt}$ as well as a comparable performance to that of HOAt regarding prevention of racemisation and coupling efficiency. In addition, Oxyma is not classified as explosive due to its slower decomposition rate. ${ }^{127}$ After the coupling reaction, the so-called capping reaction with acetic acid anhydride $\left(\mathrm{Ac}_{2} \mathrm{O}\right.$, $20 \%$ in NMP) is performed, to deactivate possible free amine functions by acylation. This can also be used in combination with DIPEA for amino acid side chain protecting groups which are especially unstable towards acids. The synthesis cycle consisting of activation, coupling, capping and deprotection is repeated as long as the desired peptide sequence is obtained. Afterwards, the peptide is cleaved from the resin and all amino acid side chains are deprotected. The procedure for the last step depends on the used resin and the amino acids within the sequence. In this work, only WANG resins were applied, which contain a $p$-alkoxybenzyl ester as linker and after cleavage lead to the free carbonic acid at the $C$-terminal end of the peptide. For this resin, cleavage is achieved by trifluoroacetic acid (TFA, $95 \%$ ) addition and to transfer the peptide sequence into the solution-phase. 124

For cleavage and complete deprotection of the amino acid side chains, a mixture of TFA (94.0\%), $\mathrm{H}_{2} \mathrm{O}(2.5 \%)$, ethanedithiole (EDT, $2.5 \%$ ) and triisopropylsilane (TIS, $1.0 \%$ ) was applied. EDT and TIS were used as so-called scavengers to prevent side reactions by cleaved fragments of protecting groups. ${ }^{[124]}$ A detailed description of the applied procedure can be found in chapter 8.4 .

\subsection{Synthesis of Adamantyl-Modified Synaptobrevin Analogues}

Based on the results from MARZ et al. [64] distinct positions of Synaptobrevin-2 SNARE motif (aa 28 - 84) were modified by introducing the unnatural amino acid building block FmocAsn(Ad)-OH (N(Ad), 4) using SPPS. In a previous work by Dr. A. GroschneR ${ }^{119]}$ a library of adamantyl-modified Synaptobrevin-2 analogues was analysed to study SNARE assembly and disassembly properties by SDS-PAGE (table 4.4). Especially derivative $\mathbf{H}$ (Syb-2 $28-84$ Q58N(Ad), 10) showed most promising disassembly properties by retarding the reaction. Based on these results, five derivatives with different affinities for complex formation were chosen for 
further quantification by fluorescence-based methods.

Table 4.4: Summary of previous results by Dr. A. GROSCHNER regarding SNARE complex assembly and disassembly properties of modified Synaptobrevin- 2 analogues analysed by SDSPAGE. 119]

\begin{tabular}{|c|c|c|c|c|}
\hline No. & Modifications & $\begin{array}{l}\text { SNARE } \\
\text { assembly }\end{array}$ & $\begin{array}{l}\text { SNARE } \\
\text { disassembly }\end{array}$ & Figure \\
\hline wt & - & $\checkmark$ & $\checkmark$ & \multirow{3}{*}{ 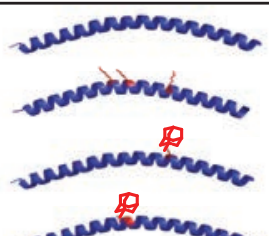 } \\
\hline Lys & D51K, E55K, D65K & $\mathbf{x}$ & & \\
\hline A & $\mathrm{D} 65 \mathrm{~N}(\mathrm{Ad})$ & $\checkmark$ & & \\
\hline B & $\mathrm{E} 55 \mathrm{~N}(\mathrm{Ad})$ & $x$ & & \\
\hline C & $\mathrm{D} 51 \mathrm{~N}(\mathrm{Ad})$ & $\checkmark$ & & \\
\hline D & E55N(Ad), D65N(Ad) & $\checkmark$ & $\checkmark$ & \\
\hline E & $\mathrm{D} 51 \mathrm{~N}(\mathrm{Ad}), \mathrm{D} 65 \mathrm{~N}(\mathrm{Ad})$ & $\checkmark$ & & \\
\hline $\mathbf{F}$ & D51N(Ad), E55N(Ad) & $\checkmark$ & $\checkmark$ & \\
\hline G & $\mathrm{D} 51 \mathrm{~N}(\mathrm{Ad}), \mathrm{E} 55 \mathrm{~N}(\mathrm{Ad}), \mathrm{D} 65 \mathrm{~N}(\mathrm{Ad})$ & X & & \\
\hline H & Q58N(Ad) & $\checkmark$ & 눈 & \\
\hline 1 & $\mathrm{E} 62 \mathrm{~N}(\mathrm{Ad})$ & $\checkmark$ & & \\
\hline
\end{tabular}

\subsubsection{Synthesis of $\mathbf{N}$-Fmoc Adamantyl Amino Acid Building Block}

(4)

The amino acid building block 4 was designed to obtain sterical hindrance on the surface of SNARE complex. This should lead to the inhibition or weakening of $\alpha$-SNAP binding. To obtain a sterically demanding side chain, the side chain of asparagine was modified with a 1-adamantyl residue. Adamantane is a cycloalkane consisting of three condensed cyclohexanes, which makes it very rigid and inflexible, resulting in a sterically demanding structure.

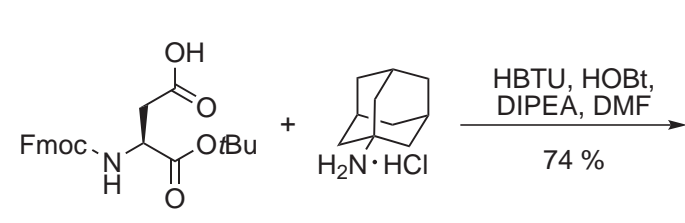

Fmoc-Asp-OtBu (1)
2

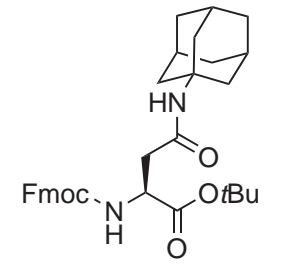

Fmoc-Asn(Ad)-OtBu (3)

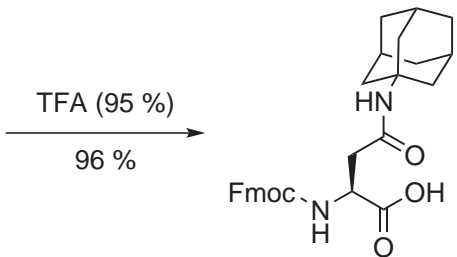

Fmoc-Asn(Ad)-OH (4)

Scheme 4.5: Synthesis scheme of unnatural amino acid building block Fmoc-Asn(Ad)-OH (4). 119$]$

Synthesis of building block 4 was accomplished in two steps, starting with Fmoc-Asp-OtBu (1) and 1-aminoadamantane (2, scheme 4.5). Using HBTU, HOBt and DIPEA in DMF, amide bond formation occurred and Fmoc-Asn(Ad)-OtBu (3) was obtained in good yields (74 \%). In 
the second step, tert-butyl ester hydrolysis was achieved by TFA (95\%) addition, and the final compound Fmoc-Asn(Ad)-OH (4) was also obtained in very good yields (96\%). 119]

\subsubsection{Synthesis of Adamantyl-Modified Synaptobrevin-2 (28 - 84)}

Modified Synaptobrevin-2 sequences including one or two modification in total were synthesised with the following positions modified: D51, E55, Q58, E62, and D65. These positions are close to the zero layer R56, and located on the surface once the SNARE complex is formed.

The following Synaptobrevin derivatives were successfully synthesised: Syb-2 (28 - 84) wild-type (6), Syb-2 (28 - 84) E55N(Ad), D65N(Ad) (7), Syb-2 (28 - 84) D51N(Ad), E55N(Ad) (8), Syb-2 (28 - 84) D51N(Ad), E55N(Ad), D65N(Ad) (9), Syb-2 (28 - 84) Q58N(Ad) (10), Syb-2 $(28$ - 84) E62N(Ad) (11) (table 4.6).

Table 4.6: Sequences of adamantyl-modified Synaptobrevin-2 derivatives $(\mathbf{7}-\mathbf{1 1})$ and Synaptobrevin-2 wild-type sequence (6). 119

\begin{tabular}{|c|c|c|}
\hline No. & Sequence & Figure \\
\hline 6 & 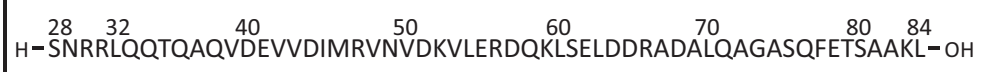 & \multirow{6}{*}{ 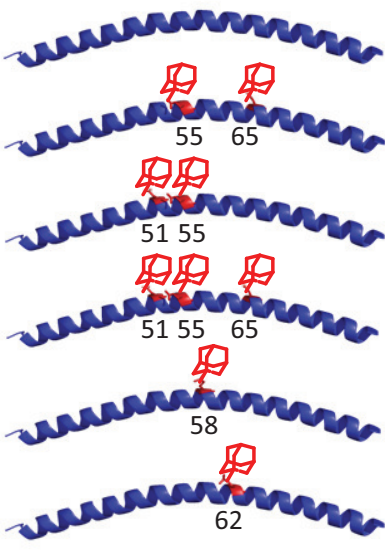 } \\
\hline 7 & \begin{tabular}{|cc} 
Ad & Ad \\
H-SNRRLQQTQAQVDEVVDIMRVNVDKVLNRDQKLSELN'NRADALQAGASQFETSAAKL-OH
\end{tabular} & \\
\hline 8 & 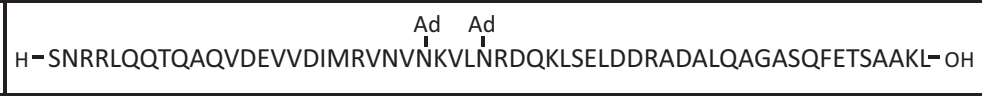 & \\
\hline 9 & $\begin{array}{|cc|}\stackrel{\text { Ad }}{\text { Ad }} & \text { Ad } \\
\text { H-SNRRLQQTQAQVDEVVDIMRVNVNNKVLNRDQKLSELNNDRADALQAGASQFETSAAKL-OH }\end{array}$ & \\
\hline 10 & $\begin{array}{c}\text { Ad } \\
\text { H-SNRRLQQTQAQVDEVVDIMRVNVDKVLERDNNKLSELDDRADALQAGASQFETSAAKL-OH }\end{array}$ & \\
\hline 11 & $\begin{array}{c}\text { Ad } \\
\text { H-SNRRLQQTQAQVDEVVDIMRVNVDKVLERDQKLSNLLDRADALQAGASQFETSAAKL-OH }\end{array}$ & \\
\hline
\end{tabular}

The strategy for synthesising the Synaptobrevin-2 SNARE motif was the following: Firstly, the $C$-terminal part Syb-2 $(66$ - 84) (5, chapter 8.6.1) was synthesised by automated and microwave-assisted SPPS using the CEM Liberty 12 peptide synthesizer, according to GSP 2 (chapter 8.4.2). The obtained resin-bound peptide 5 was subdivided into several batches to continue synthesis of the different derivatives (peptide $\mathbf{6}-\mathbf{1 1}$, chapter 8.6.2 - 8.6.7) by manual SPPS according to GSP 1 (chapter 8.4.1). Due to the length of the peptide sequence, double coupling $(2 \times 30 \mathrm{~min})$ was performed and from amino acid D51 on the coupling time was elongated to $2 \times 45 \mathrm{~min}$ except for R47 $(2 \times 1 \mathrm{~h})$ and R31, R30 ( $3 \times 1 \mathrm{~h})$. After complete synthesis, the peptides $\mathbf{6}-\mathbf{1 1}$ were cleaved from the solid-support and completely deprotected according to GSP 3 (chapter 8.4.3). After purification by HPLC, the influence of the adamantyl-modified Synaptobrevin-2 analogues on the SNARE complex formation as well as disassembly reaction was analysed, described in chapter 4.5. The technique used for monitoring disassembly reactions is described in detail in the following chapter. 


\subsection{Fluorescence Anisotropy}

Fluorescence anisotropy as well as fluorescence resonance energy transfer (FRET) are widely used tool to investigate biochemical processes. Compared to FRET where two fluorophores within the investigated system are needed in a certain distance, the advantage of fluorescence anisotropy is that only one fluorophore is needed without any distance dependency.

For measurements of fluorescence anisotropy, polarised light is used for excitation which leads to polarised emission from many samples. The anisotropy $(r)$ describes the dimension of polarization of the emitted light. For example, a sample with complete polarised emission exhibits a non zero anisotropy. But this state can not be observed for homogeneous unoriented probes. Anisotropy is based on the relative orientation of the transition moments for absorption and emission within the fluorophore structure. In a homogeneous solution, they are orientated randomly in the ground-state. Upon excitation with polarised light, fluorophores with their transition moment of absorption oriented parallel to the electric vector of the light, exhibit the highest probability to be excited. Consequently, fluorophores are partially oriented in the excited state. 128,129

In scheme 4.7 the experimental setup for measuring fluorescence anisotropy is shown. The light beam for excitation is directed from the source along the $x$-axis with the linear polarisation oriented parallel to the $z$-axis. In the origin, a hypothetical molecule is placed with its transition dipole for absorption oriented at an angle of $\theta$ towards the $z$-axis. $\gamma$ indicates the angle between the absorbing and emitting transition dipole of the molecule. Along the $y$-axis, the emitted fluorescence is measured by a polarizer which detects the intensities with polarization parallel $\left(I_{\|}\right)$and perpendicular $\left(I_{\perp}\right)$ to the polarization of the exciting light. By these intensities, the depolarization in terms of anisotropy $(r)$ can be calculated ${ }^{129}$ :

$$
r=\frac{I_{\|}-I_{\perp}}{I_{\|}+2 I_{\perp}}
$$

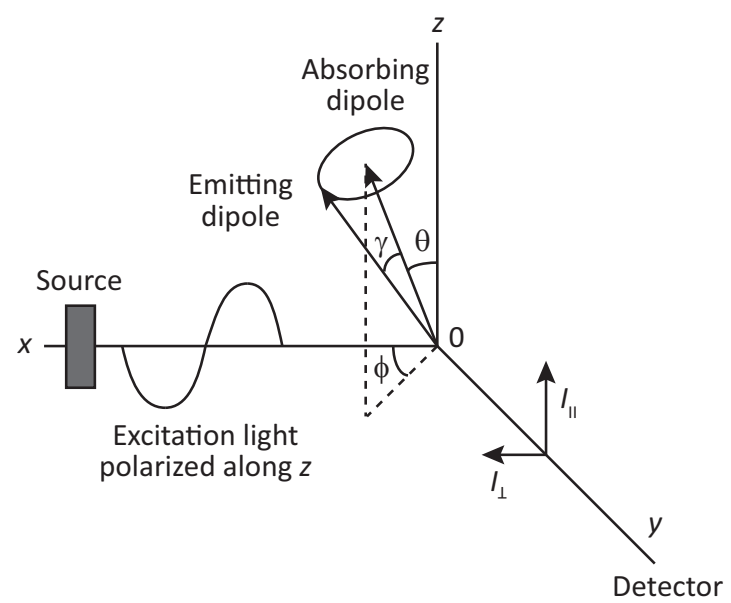

Scheme 4.7: Experimental setup for fluorescence anisotropy measurements. 129 
The resulting value is dimensionless and independent of the total intensity and the fluorophore concentration. In addition, anisotropy depends on the rotational motion of the fluorophore during its lifetime at the excited state, since rotational changes influence the orientation of the transition moments. This dependence is used for many applications in biochemical research, due to the fact that the rotational diffusion of biomolecules and the decay time of many fluorophores are on a comparable timescale. As a result direct measurement of the factors which alter the rotational movement of a biomolecule is possible. For example, the anisotropy is decreased by increasing rotational diffusion and internal flexibility. The depolarization caused by rotational diffusion of spherical rotors is described by the PERKIN equation ${ }^{128}$ :

$$
\frac{r_{0}}{r}=1+\tau / \theta=1+6 D \tau
$$

with the fluoroscence lifetime $\tau$, the rotational correlation time $\theta$ and the rotational diffusion coefficient $D$. In the case of a much larger correlation time than lifetime $(\theta \gg \tau)$, the measured anisotropy $(r)$ is equal to the fundamental anisotropy $\left(r_{0}\right)$. The other way round $(\theta \ll \tau)$, the anisotropy is zero. For globular proteins, a relation between correlation time and molecular weight $(M)$ is given by

$$
\theta=\frac{\eta V}{R T}=\frac{\eta M}{R T}(\bar{v}+h)
$$

with the viscosity $\eta$, the volume of the rotating unit $V$, the gas constant $R$, the temperature $T$, the specific volume of the protein $\bar{v}$ and the hydration $h .128$ 129] The equation shows the dependency of rotational movement of the sample on the molecular weight, volume and hydration, and therefore the possibility to measure changes in these parameters by fluorescence anisotropy. For example, an increase of molecular weight by a binding event leads to a decrease of the lifetime/rotational correlation time ratio, and consequently, to an increase in anisotropy.

Two methods are available for fluorescence anisotropy measurements: L-format or single-channel method (scheme $4.8 \mathrm{a}$ ) and T-format or two-channel method (scheme $4.8 \mathrm{c}$ ). In the first case, a single emission channel is used with a monochromator in between which has usually different transmission efficiencies for vertically and horizontally polarised light. In the second case, two separate detectors are used for measuring the intensities of parallel and perpendicular polarised light simultaneously. 128

For the L-format method, the detected intensities (e.g. $I_{V V}, I_{V H}$ for vertically polarised excitation $(\mathrm{V})$ and horizontally polarised emission $(\mathrm{H})$ ) differ from the desired intensities $I_{\|}$ and $I_{\perp}$ by a factor $G$ :

$$
\frac{I_{V V}}{I_{V H}}=G \frac{I_{\|}}{I_{\perp}}
$$

and the anisotropy $(r)$ can be calculated as follows:

$$
r=\frac{I_{V V}-G I_{V H}}{I_{V V}+2 G I_{V H}}
$$


a
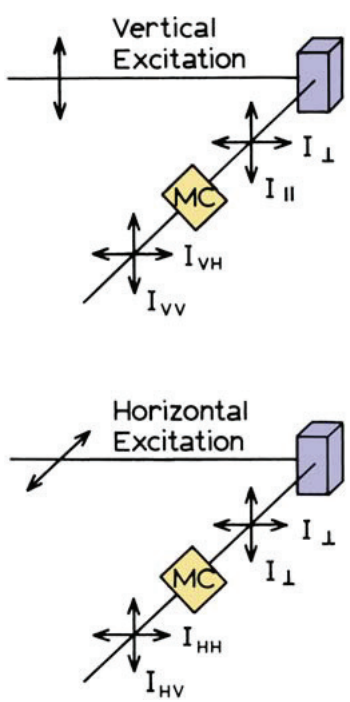
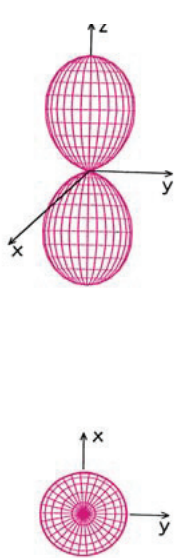
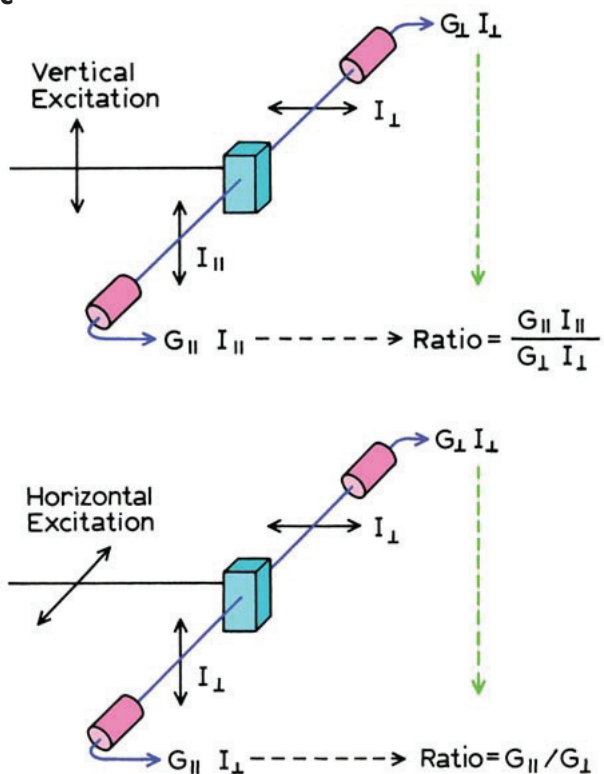

Scheme 4.8: Schematics of different methods for fluorescence anisotropy measurements: (a) L-format or single-channel measurements with monochromators (MC) and (c) T-Format or two-channel measurements. (b) Shapes of the excited-state distributions. 128 ,

Hence, to calculate the anisotropy $(r)$, it is necessary to determine the $G$ factor, which includes the sensitivities of the emission polarisers. This can be done by using horizontally polarised excitation, due to the fact that the horizontally and vertically polarised components are equal and also proportional to $I_{\perp}$ (scheme $4.8 \mathrm{a}$ ). In that case, any intensity differences in $I_{H V}$ and $I_{H H}$ result from the detection system which is represented in the $G$ factor:

$$
G=\frac{I_{H V}}{I_{H H}}
$$

Without correction of the calculated anisotropy by determination of the $G$ factor, the measured values will be incorrect. 128

For the T-format method, which was applied in this work, two separate detectors are used. Due to no change in the emission polarisers, the sensitivity of each channel remains equal during the measurements. But nevertheless, the relative sensitivity of the two detectors has to be determined. Firstly, with vertical polarised excitation, the ratio of parallel and perpendicular signals $\left(R_{V}\right)$ is measured:

$$
R_{V}=\frac{G_{\|} I_{\|}}{G_{\perp} I_{\perp}}
$$

with the sensitivities of the parallel $G_{\|}$and perpendicular $G_{\perp}$ channel. With horizontally polarised excitation, both emission channels detect $I_{\perp}$ and the ratio of sensitivities can be determined by the ratio of the signals $\left(R_{H}\right)$ :

$$
R_{H}=\frac{G_{\|}}{G_{\perp}} \text { leading to } \frac{R_{V}}{R_{H}}=\frac{I_{\|}}{I_{\perp}},
$$

which can be used to calculate the anisotropy $(r)$ : 


$$
r=\frac{I_{\|} / I_{\perp}-1}{I_{\|} / I_{\perp}+2}
$$

Due to the simultaneous measurement, possible fluctuations in the intensities are cancelled with the T-format method and, thus, was preferentially used in the past. Today, the only advantage of $\mathrm{T}$-format measurements is the decreased time for data acquisition. 128 ,

\subsection{SNARE Assembly and Disassembly Properties of Adamantyl-Modified Synaptobrevin Analogues}

Adamantyl-modified Synaptobrevin-2 analogues were investigated regarding the SNARE complex assembly and disassembly properties. The natural counterparts (Syntaxin-1A and SNAP-25) for SNARE complex formation were expressed and purified by Dr. H. YAVUZ, Prof. R. JAHN's work group, Department of Neurobiology, Max-Planck-Institute for Biophysical Chemistry Göttingen (chapter 8.3.5) as well as co-floatation assays and fluorescence anisotropy experiments were conducted in collaboration.

\subsubsection{SNARE Complex Formation and Reconstitution into SUVs and Co-Floatation Assay}

For SNARE complex formation of the adamantyl-modified Synaptobrevin-2 analogues with the natural counterparts, the following peptides were mixed and incubated for $2 \mathrm{~h}$ at room temperature under exclusion of light (chapter 8.3.6): Syntaxin-1A $(183-288)$, Oregon Green (OG)-labelled SNAP-25 $(1$ - 206) C130-OG, Synaptobrevin-2 $(28$ - 84) X $(1.0 / 1.0 / 1.5, n / n / n)$. For these experiments, Syntaxin-1A sequence containing the transmembrane domain was used for further reconstitution into vesicles. To solubilise membrane peptides/proteins in aqueous solution, addition of detergents is necessary. Consequently, SNARE complex formation was conducted in a detergent containing HEPES buffer $(20 \mathrm{~mm}, \mathrm{NaCl}(300 \mathrm{~mm})$, TCEP $(0.1 \mathrm{~mm})$, CHAPS $(2 \%))$ at $\mathrm{pH} 7.4$.

Due to the membrane-dependent efficiency of $\alpha$-SNAP binding to the SNARE complex, all investigated complexes were reconstituted into small unilamellar vesicles (SUVs) by the co-micellisation method (chapter 8.3.7). 11 18 With this method, SUVs are obtained by mixing lipids and proteins in the detergent containing buffer followed by the removal of the detergent by size-exclusion chromatography. The used lipid mix consisted of phosphatidylcholine (PC, $50 \%$ ), phosphatidylethanolamine (PE, $20 \%$ ), phosphatidylserine (PS, $20 \%$ ) and cholesterol $(10 \%)$ in HEPES buffer $(20 \mathrm{~mm}, \mathrm{KCl}(150 \mathrm{~mm})$, EDTA $(1 \mathrm{~mm})$, DTT (1 mM), NaCholate $(5 \%, w / v), \mathrm{pH} 7.4)$. Lipid mix and protein mix were combined in a protein to lipid ratio of $1 / 1000$. The mixture was applied to a size-exclusion column equilibrated with a detergent free HEPES buffer $(20 \mathrm{~mm}, \mathrm{KCl}(150 \mathrm{~mm})$, EDTA $(1 \mathrm{mM})$, DTT $(1 \mathrm{mM}), \mathrm{pH} 7.4)$ and the 
vesicle fractions were collected. Due to OG-labelled SNARE complexes, the collected fraction with highest emission under UV lamp was chosen for further experiments (see chapter 8.3.7).

For investigating the quantity of reconstituted SNARE complexes, co-floatation assays were performed. 18 60 With this method, proteoliposomes are subjected to a density gradient in which the vesicles float on top constituting the lowest density fraction and all the non-reconstituted peptides are localised at the bottom constituting the highest density fraction.

The fractions obtained from size-exclusion chromatography were used for co-floatation assays (chapter 8.3.8). The eight aliquots (F1 - F8) isolated after the co-floatation assay were analysed by SDS-PAGE (figure 4.9), whereby F1 constituted of the lowest density fraction and F8 of the highest density fraction.

\section{Syb-2 (28 - 84) wild-type (6)}

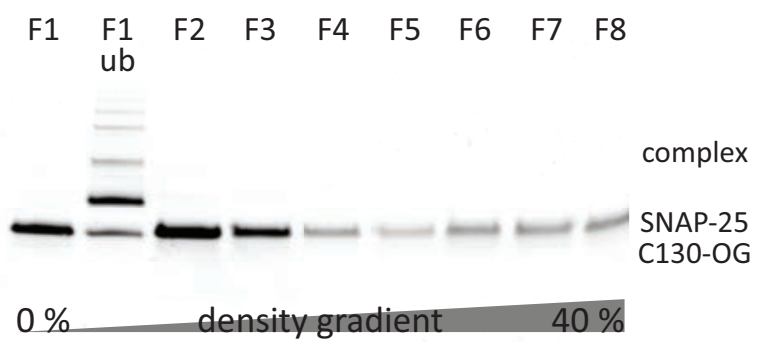

Syb-2 (28 - 84) D51N(Ad), E55N(Ad) (8)

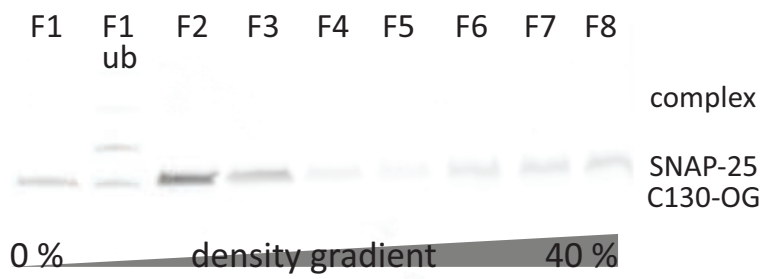

Syb-2 (28 - 84) E62N(Ad) (11)

$\begin{array}{lllllllll}\text { F1 } & \text { F1 } & \text { F2 } & \text { F3 } & \text { F4 } & \text { F5 } & \text { F6 } & \text { F7 } & \text { F8 }\end{array}$

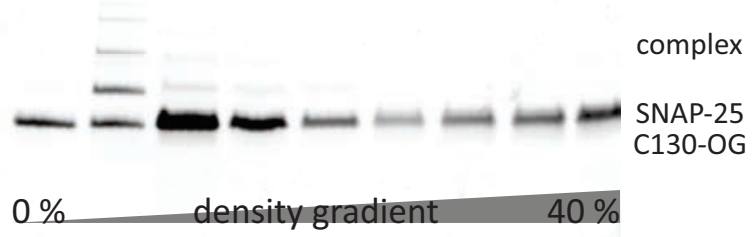

Syb-2 (28 - 84) E55N(Ad), D65N(Ad) (7)
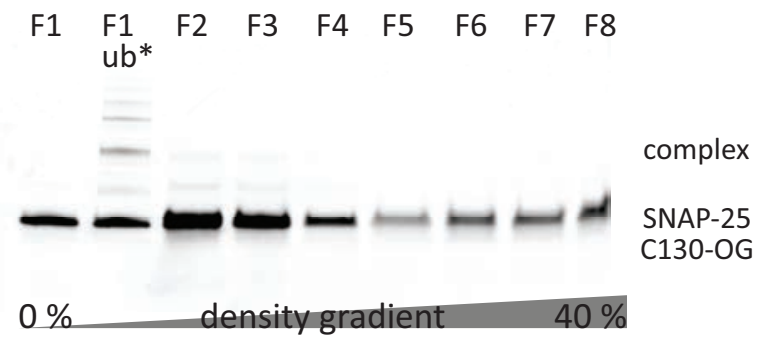

Syb-2 (28 - 84) Q58N(Ad) (10)

$\begin{array}{lllllllll}\text { F1 } & \text { F1 } & \text { F2 } & \text { F3 } & \text { F4 } & \text { F5 } & \text { F6 } & \text { F7 } & \text { F8 }\end{array}$

complex

SNAP-25 C130-OG

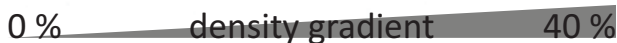

Figure 4.9: Fractions (F1 - F8) obtained by Co-floatation assays analysed by SDS-PAGE for SNARE complexes containing peptides $\mathbf{6}, \mathbf{7}, \mathbf{8}, \mathbf{1 0}$, and $\mathbf{1 1}$. All fractions were boiled in sample buffer except for fraction F1, which was subdivided into two samples, one was boiled and the second not boiled (ub). *Sample was boiled for few seconds.

In addition, F1 was divided into two samples: one was boiled and the other was not boiled 
(ub) before loading on the gel. The ternary SNARE complex is resistant towards SDS but not stable at that high temperatures. Consequently, to estimate whether complexes were formed, an unboiled fraction was needed. For all SNARE complexes, an upper band ( $35-40 \mathrm{kDa})$ in the unboiled top fraction F1 could be observed. Meaning, all investigated Synaptobrevin-2 analogues were able to form the ternary SNARE complex and were successfully reconstituted into SUVs. In the case of SNARE complex containing Syb-2 (28 - 84) D51N(Ad), E55N(Ad) (8), fluorescence intensity of the bands was low due to the overall low concentration used in comparison to the other derivatives.

The two top fractions (F1 and F2) of each co-floatation assay were combined and used for the disassembly experiments by fluorescence anisotropy (chapter 4.5.3). Thereby, the assay was also used as a purification method prior to disassembly reaction.

\subsubsection{Fluorescence Anisotropy Experiments of SNARE Complex Formation}

SNARE assembly properties of adamantyl-modified Synaptobrevin-2 derivatives were analysed by fluorescence anisotropy using a soluble, truncated SNARE complex consisting of Syntaxin-1A (183 - 262), SNAP-25 (1 - 206) no Cys mutant, and Synaptobrevin-2 (49 - 96) C79-OG. Previous publications showed that, with this stabilised acceptor complex ( $\Delta \mathrm{N}$ complex $)$, SNARE complex formation properties can be measured indirectly by displacement of the Syb-2 fragment. 1118 60 SNARE complex assembly was shown to start at the $N$-terminal part of the SNARE motifs. Therefore, the Synaptobrevin-2 comprising a longer $N$-terminal part of the SNARE motif can displace the $C$-terminal Syb-2 fragment of the $\Delta \mathrm{N}$ complex (figure 4.10a). The binding event can be monitored by time-dependent fluorescence anisotropy: The labelled Syb-2 fragment assembled in the $\Delta \mathrm{N}$ complex exhibits a certain anisotropy level, whereas the free labelled Syb-2 fragment shows a distinct lower anisotropy level. Upon addition of a longer Syb-2 peptide, the anisotropy decreases due to the displacement event. Based on the time-dependent anisotropy curve, it is possible to determine the kinetics of the displacement reaction. $11,18,60$

The following synthesised Synaptobrevin- 2 derivatives were analysed at $20{ }^{\circ} \mathrm{C}$ by time-dependent fluorescence anisotropy (figure 4.10 b): Syb-2 (28 - 84) wild-type (6), Syb-2 (28 - 84) E55N(Ad), D65N(Ad) (7), and Syb-2 (28 - 84) Q58N(Ad) (10). The first 1.5 eq of Syb-2 derivatives $(120 \mathrm{nM})$ were added to the $\Delta \mathrm{N}$ complex $(80 \mathrm{nM})$ after $\sim 200 \mathrm{~s}$ of measurement. The concentration of Syb-2 was subsequently increased to 2.25 eq (180 nM) after $\sim 575 \mathrm{~s}$ of measurement. However, no significant change in reaction speed was observed by the second addition, concluding that 1.50 eq excess of Syb-2 is sufficient for SNARE complex formation. For direct comparison of the kinetics with the shorter wild-type sequence, Syb-2 (1 - 96) was also added to the $\Delta \mathrm{N}$ complex. It was shown that Syb-2 (28-84) wild-type (6, black curve) 

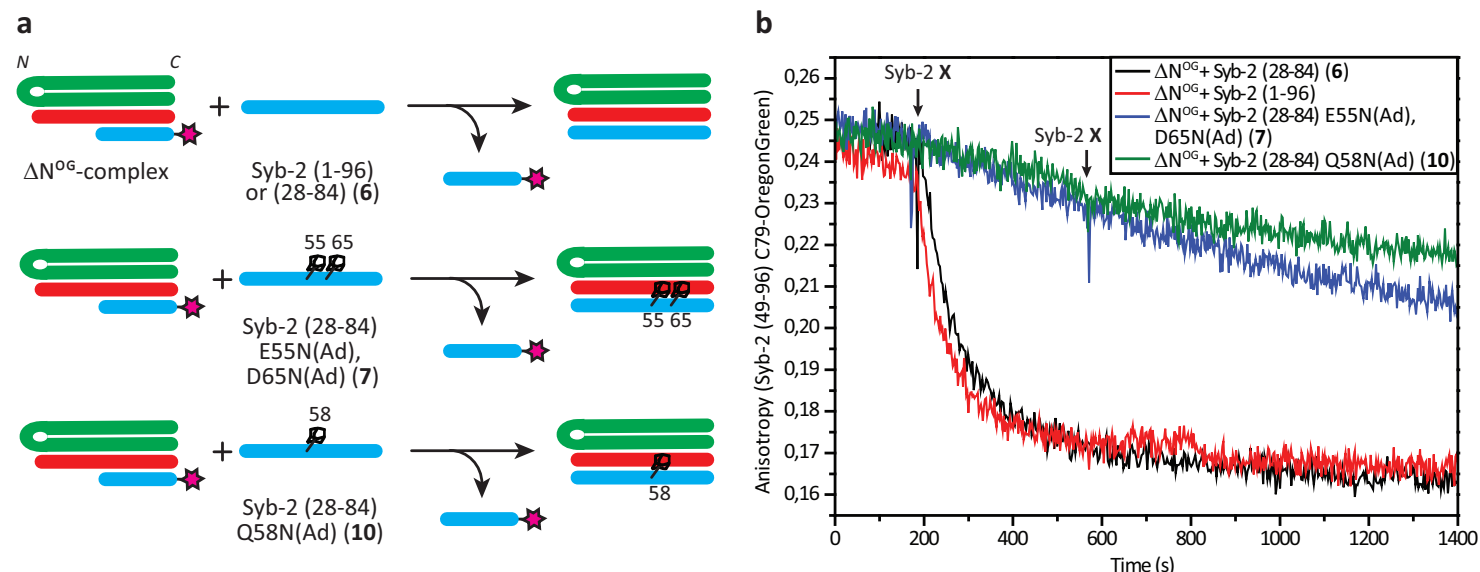

Figure 4.10: SNARE assembly experiments with OG-labelled truncated SNARE complex $(\Delta N$ complex). (a) Scheme of performed experiments with $\Delta \mathrm{N}$ complex consisting of Syntaxin-1A (red), SNAP-25 (green) and Syb-2 fragment (blue) labelled with OG (pink star). By addition of synthesised Synaptobrevin-2 sequences, the short Synaptobrevin-2 fragment will be displaced. (b) fluorescence anisotropy experiments with $\Delta \mathrm{N}$ complexes. Additions of corresponding Synaptobrevin-2 derivative (Syb-2 (28 - 84) wild-type (6, black), Syb-2 (1 - 96) (red), Syb-2 $(28-84) \mathrm{E} 55 \mathrm{~N}(\mathrm{Ad}), \mathrm{D} 65 \mathrm{~N}(\mathrm{Ad})(7)$ (blue), or Syb-2 (28 - 84) Q58N(Ad) (10) (green)) are indicated by the arrows. First addition of Syb-2 derivatives $(120 \mathrm{nM})$ to the OG-labelled $\Delta \mathrm{N}$ complex $(80 \mathrm{nM})$ was conducted after $\sim 200 \mathrm{~s}$ of measurement, followed by a second addition of Syb-2 derivatives to increase the concentration to $180 \mathrm{nM}$ after $\sim 575 \mathrm{~s}$ of measurement. Synaptobrevin-2 fragment displacement is traced by the decrease in anisotropy.

exhibits a similar kinetic profile to displace the Syb-2 fragment as the longer version Syb-2 $(1-96)$ wild-type (red curve). As a consequence, Syb-2 (28 - 84) wild-type containing the whole SNARE motif sequence is comparable in its properties to Syb-2 $(1-96)$. In addition, two of the adamantyl-modified Synaptobrevin-2 derivatives were investigated. Syb-2 (28 - 84) E55N(Ad), D65N(Ad) (7) (blue curve) as well as Syb-2 (28 - 84) Q58N(Ad) (10) (green curve) showed clearly retarded fragment displacement. Even addition of further excess (2.25 eq) after $\sim 575$ s showed no acceleration of the reaction.

To summarise, the synthesised wild-type sequence comprising only the SNARE motif of Synaptobrevin-2 is sufficient as it is comparable in its SNARE assembly properties to the longer version. Secondly, adamantyl modifications showed clear impairment of SNARE complex formation using $\Delta N$ complexes. The positions of the modifications ( $555 N(A d), Q 58 N(A d)$, $\mathrm{D} 65 \mathrm{~N}(\mathrm{Ad}))$ are close to the beginning of the $C$-terminal Syb-2 fragment $(49-96)$ which might be the reason for the clear retardation of fragment displacement. Probably, SNARE complex formation with adamantyl-modified Synaptobrevin-2 species from monomers did not get impaired to this extent, as SNARE complexes were successfully assembled and reconstituted after $2 \mathrm{~h}$ incubation with monomeric species (chapter 4.5.1). 


\subsubsection{Fluorescence Anisotropy Experiments of SNARE Disassembly Reaction}

All disassembly reactions were performed in disassembly buffer consisting of HEPES (50 mM), potassium acetate (KAc, $20 \mathrm{mM}$ ), potassium glutamate (KGlu, $120 \mathrm{~mm}$ ) at $\mathrm{pH} 7.4$ and $37{ }^{\circ} \mathrm{C}$. SNARE complexes reconstituted into SUVs and isolated from the two top fractions (F1, F2) obtained during the co-floatation assays were used (chapter 4.5.1). Time-dependent fluorescence anisotropy measurements were performed using Fluorolog 3 spectrometer. Excitation and emission wavelengths were set to $488 \mathrm{~nm}$ and $520 \mathrm{~nm}$ for Oregon Green (chapter 8.3.9).

Firstly, influence of co-floatation assay on anisotropy measurements was analysed in the case of SNARE complex containing Syb-2 (28 - 84) wild-type (6) (figure 4.11 a). Therefore, samples before and after the assay were used for disassembly reaction. In general, the disassembly reaction could be observed by an increase in anisotropy upon $\alpha$-SNAP binding to the SNARE complex (figure 4.11 a, $\sim 200 \mathrm{~s}$ ). After subsequent addition of NSF ( 400 s), an decrease in anisotropy below the initial level was monitored, which indicates complete disassembly of the SNARE complex into the individual peptides.

The comparison of the samples before and after the co-floatation assay shows a clearer increase in anisotropy upon $\alpha$-SNAP binding for the sample after the assay (figure 4.11 a, black and green curves). Meaning, the separation of non-reconstituted peptides by co-floatation assay is reasonable for these experiments.

Furthermore, control experiments for the disassembly reaction were conducted. In the first control experiment buffer was added instead of $\mathrm{MgCl}_{2}$ (figure 4.11 a, no $\mathrm{Mg}$ (control), red curve). Since $\mathrm{Mg}^{2+}$ is necessary for ATP hydrolysis by NSF, the enzyme is not able to disassemble the SNARE complex without addition of $\mathrm{MgCl}_{2}$. This was exactly observed in the control experiment: $\alpha$-SNAP was still able to bind to the SNARE complex (increase in anisotropy), but after NSF addition no decrease in anisotropy was observed and the SNARE complex was not disassembled.

In the second control experiment buffer was added instead of $\alpha$-SNAP (figure 4.11 a, no $\alpha$-SNAP (control), blue curve). As mentioned before, NSF is not able to bind to the SNARE complex without prior binding of $\alpha$-SNAP. In other words, without $\alpha$-SNAP in the solution, NSF will not disassemble the SNARE complex. This was confirmed by the observation made in the control experiment: wherein, no increase in anisotropy in the absence of $\alpha$-SNAP was observed, and no decrease in anisotropy after NSF addition.

Disassembly experiments of SNARE complexes containing adamantyl-modified Synaptobrevin-2 analogues were performed exactly like in the wild-type case described above. For the SNARE complex containing Syb-2 (28 - 84) E55N(Ad), D65N(Ad) (7) as well as for the SNARE complex containing Syb-2 (28 - 84) Q58N(Ad) (10), the disassembly reaction proceeded 
similar to the wild-type (figure 4.11 b and c, black curves): An increase in anisotropy was observed upon $\alpha$-SNAP binding and a decrease in anisotropy was observed upon NSF addition. In spite of the adamantyl modifications, $\alpha$-SNAPs were still able to bind to the SNARE complex surfaces, and NSF disassembled the SNARE complexes. The two control experiments were similar to the wild-type experiments (figure 4.11 b and c, red and blue curves).

The overlay of all performed disassembly experiments (figure 4.11 d) shows that none of the adamantyl modification had an influence on the $\alpha$-SNAP binding to the SNARE complex surfaces, and all the SNARE complexes were disassembled by the NSF/ $\alpha$-SNAP machinery.

a $\quad$ Syb-2 (28 - 84) wild-type (6)

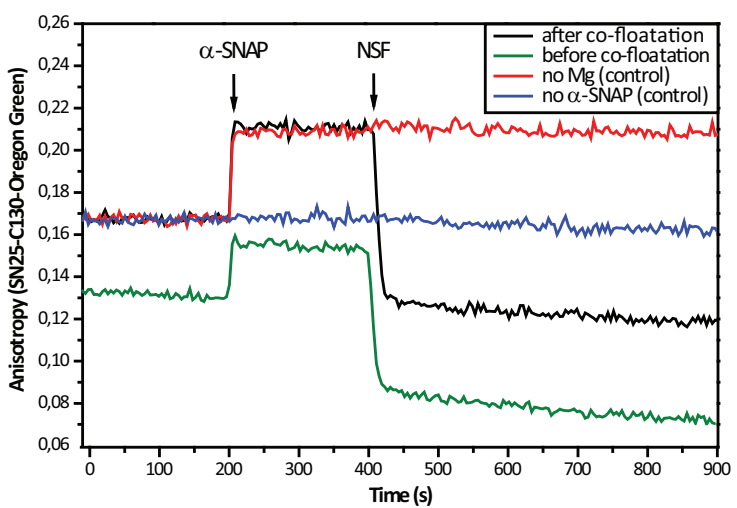

C $\quad$ Syb-2 (28 - 84) Q58N(Ad) (10)

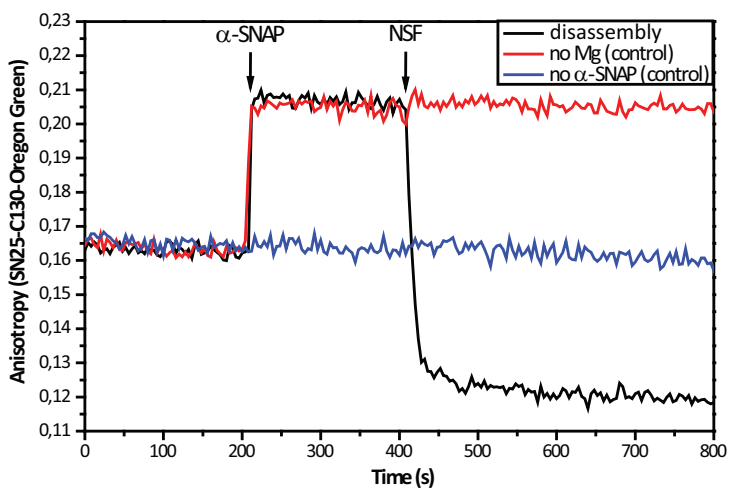

b $\quad$ Syb-2 (28 - 84) E55N(Ad), D65N(Ad) (7)

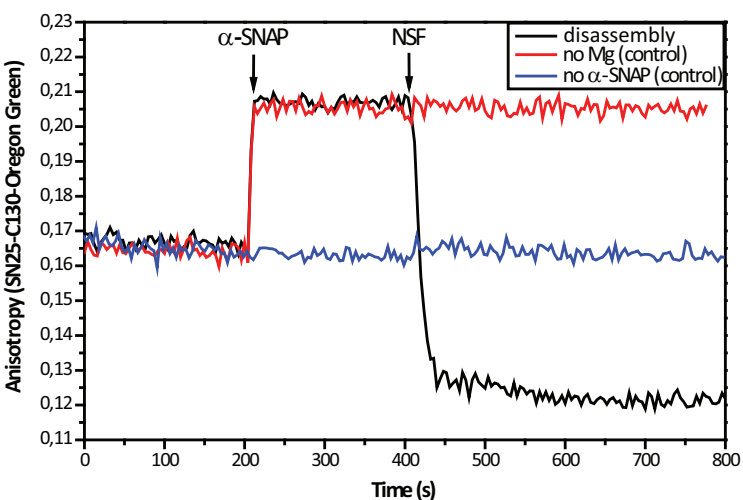

d

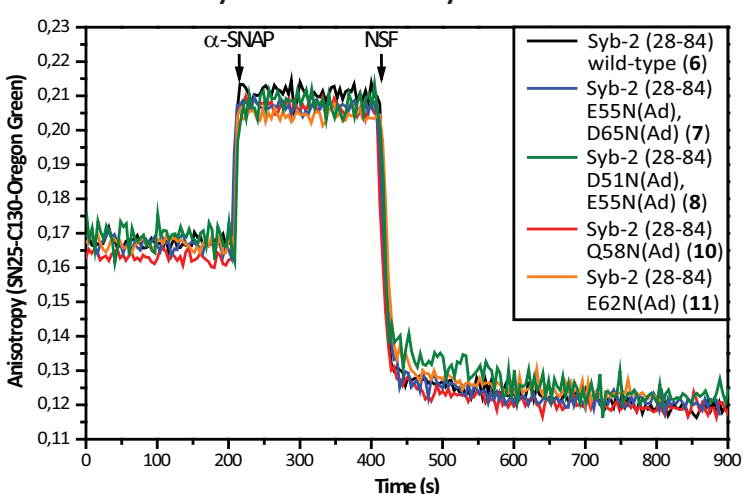

Figure 4.11: Disassembly reaction of SNARE complex containing synthesised Syb-2 $(28-84)$ derivatives (Syb-2 (28 - 84) wild-type (6), Syb-2 (28 - 84) E55N(Ad), D65N(Ad) (7), Syb-2 $(28$ - 84) D51N(Ad), E55N(Ad) (8), Syb-28 (28 - 84) Q58N(Ad) (10), and Syb-2 (28 - 84) E62N(Ad) (11)) analysed by fluorescence anisotropy. (a) Disassembly experiment of SNARE complex containing Syb-2 $(28$ - 84) wild-type (6). Samples used before (green) and after (black) co-floatation assay as well as control experiments with buffer addition instead of $\mathrm{MgCl}_{2}$ addition (red) and instead of $\alpha$-SNAP addition (blue). Addition of $\alpha$-SNAP and NSF is indicated by corresponding arrows. (b) Disassembly experiments of SNARE complex containing adamantylmodified Syb-2 (28 - 84) E55N(Ad), D65N(Ad) (7, black) and (c) Syb-28 (28 - 84) Q58N(Ad) (10, black) including corresponding control experiments (red and blue). (d) Overlay of all performed disassembly reactions excluding control experiments. 


\subsection{Complexin Binding Studies with Adamantyl-Modified Synaptobrevin Analogues}

Complexin binding studies of adamantyl-modified Synaptobrevin-2 analogues-containing SNARE complexes were carried out due to the fact that $\alpha$-SNAP and Complexin compete for SNARE complex binding. ${ }^{59}$ 67] Specifically, Synaptobrevin-2 residues on the SNARE complex surface interacting with $\alpha$-SNAP (D51, E55, and D65) ${ }^{64}$ and with Complexin (D57, D64, D65, and D68) ${ }^{74}$ are in close proximity or even match (see chapter 2.7.4).

Consequently, some of the synthesised adamantyl-modified Synaptobrevin derivatives potentially influence the binding of Complexin to the SNARE complex surface. To investigate this issue, time-dependent fluorescence anisotropy experiments for visualisation of Complexin binding were performed. First of all, a test reaction for detection of Complexin binding by fluorescence anisotropy was conducted as a proof of principle (chapter 4.6.1). Afterwards, SNARE complexes containing synthesised Syb-2 (28 - 84) wild-type (6) were investigated regarding their Complexin binding ability (chapter 4.6.2) and subsequently, Complexin binding to SNARE complexes containing Syb-2 (28 - 84) E55N(Ad), D65N(Ad) (7) and Synaptobrevin-2 (28 - 84) Q58N(Ad) (10) was analysed (chapter 4.6.3). These two derivatives were chosen due to a match or close proximity between their site of modification and the identified interaction sites. Furthermore, SNARE complex formation especially for Synaptobrevin-2 (28 - 84) Q58N(Ad) (10) was verified by SDS-PAGE as a result of the problems associated with SNARE assembly.

SNARE complex formation with kindly provided natural counterparts, SDS-PAGE as well as fluorescence anisotropy experiments were conducted in collaboration with Dr. H. YAVUZ, Prof. R. JAHN's work group, Department of Neurobiology, Max-Planck-Institute for Biophysical Chemistry, Göttingen.

All Complexin binding reactions were performed as stated in chapter 8.3 .9 using HEPES buffer and the Fluorolog 3 spectrometer with excitation and emission wavelength set to $488 \mathrm{~nm}$ and $520 \mathrm{~nm}$, respectively, for Oregon Green.

\subsubsection{Fluorescence Anisotropy Experiments of Complexin Binding - Proof of Principle}

First, a test experiment was performed as a proof of principle, to study if the binding of Complexin to the ternary SNARE complex could be measured by fluorescence anisotropy (figure 4.12). Due to available OG-labelled, purified ternary SNARE complexes (SNAP-25 $(1-206)$ C130-OG, Syntaxin-1A $(183-288)$, Synaptobrevin-2 $(1-96))$, it was first tested, whether by addition of unlabelled Complexin I $(1-134)$ a decrease in anisotropy was detectable (figure 4.12 a, top). Unfortunately, this was not the case (data not shown). 

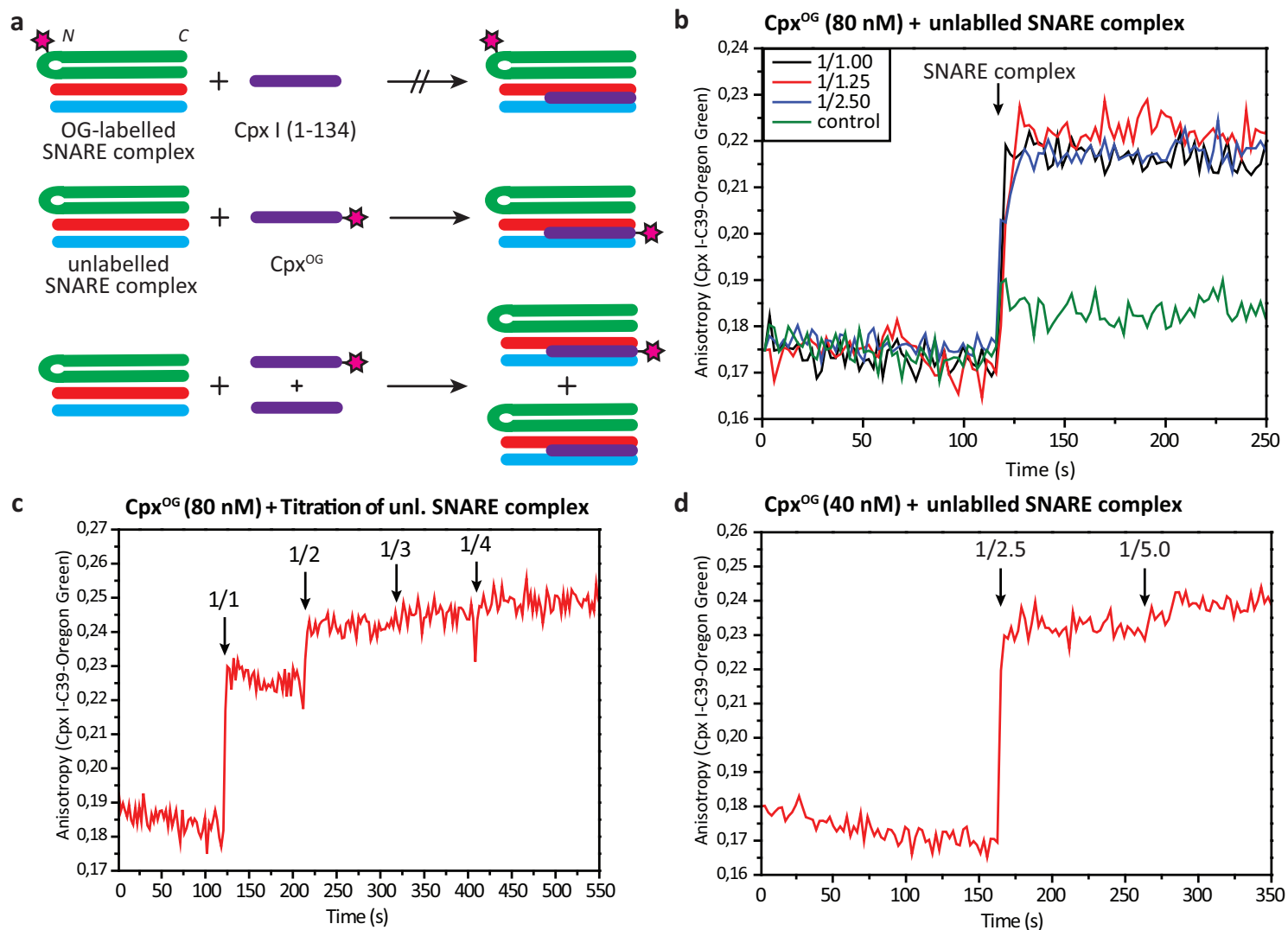

Figure 4.12: Complexin binding studies by fluorescence anisotropy as a proof of principle. (a) Schemes of performed Complexin binding reactions with same color code as in figure 4.10 except for Complexin (purple). (b) Complexin binding reactions measured by fluorescence anisotropy. (a, top) Binding event of OG-labelled SNARE complex with unlabelled Cpx I $(1-134)$ resulted in no observable change in Anisotropy. (a, middle), and $\mathbf{b}$ ) By addition of unlabelled SNARE complex to OG-labelled Cpx I $(1-134) \mathrm{E} 39 \mathrm{C}, \mathrm{C} 105 \mathrm{~S}$, the binding event was detectable. Addition is indicated by the arrow. Different molar ratios of $\mathrm{Cpx}^{\mathrm{OG}}(80 \mathrm{nM})$ and SNARE complex were tested. (a, bottom and b) Control experiment: Excess of unlabelled Cpx (11.3 eq) were combined with $\mathrm{Cpx}{ }^{\mathrm{OG}}(80 \mathrm{nM})$ prior to the addition of SNARE complex (320 nM). (c) Titration experiment of $\mathrm{Cpx} \mathrm{OG}^{\mathrm{OG}}$ and unlabelled SNARE complex. (d) Complexin binding reaction with lower $\mathrm{Cpx}{ }^{\mathrm{OG}}$ concentration (40 $\mathrm{nM})$.

Consequently, a cysteine mutant of Complexin (Cpx I (1 - 134) E39C, C105S) was expressed and purified as previously published ${ }^{72}$, and labelled with Oregon Green. Finally, addition of unlabelled ternary SNARE complex (SNAP-25 (1 -206) no Cys mutant, Syntaxin-1A (183 - 288), Synaptobrevin-2 $(1-96) \mathrm{S} 28 \mathrm{C})$ to the OG-labelled Complexin $\left(\mathrm{Cpx}^{\mathrm{OG}}\right)$ resulted in a clear increase in anisotropy (figure $4.12 \mathrm{a}$, middle, and $\mathrm{b}$ ), as previously described ${ }^{72}$. Furthermore, when different molar ratios of $\mathrm{Cpx}{ }^{\mathrm{OG}}$ and SNARE complex were tested, it was found that the addition of SNARE complex $(80 \mathrm{nM})$ to $\mathrm{Cpx}^{\mathrm{OG}}(80 \mathrm{nM})$ in a ratio of $1 / 1$ showed already a distinct increase in anisotropy (figure $4.12 \mathrm{~b}$, black curve). Comparable results were provided by ratios of $1 / 1.25$ for $\mathrm{Cpx}^{\mathrm{OG}}$ (80 $\mathrm{nM}$ )/SNARE complex. (100 nM) and 1/2.50 for $\mathrm{Cpx}$ OG (80 nM)/SNARE complex (200 nM) (figure 4.12 b, red and blue curves). In addition, a control experiment was performed by addition of SNARE complex (320 nM) to a combination of $\mathrm{Cpx}{ }^{\mathrm{OG}}(80 \mathrm{nM})$ and excess unlabelled $\mathrm{Cpx}(900 \mathrm{nM}, 11.3 \mathrm{eq})$ (figure $4.12 \mathrm{a}$, bottom, and b, 
green curve). Only a slight increase in anisotropy was observed which might be because of excess SNARE complex available (molar ratio $1 / 4$ ) in the reaction, but overall, the increase is clearly smaller compared to experiments without unlablled $\mathrm{Cpx}$. This experiment shows that unlabelled $\mathrm{Cpx}$ blocks the binding site for $\mathrm{Cpx}^{\mathrm{OG}}$ and that the increase in anisotropy in the other experiments is based on $\mathrm{Cpx}{ }^{\mathrm{OG}}$ binding event to the SNARE complex.

Furthermore, a titration experiment was performed by consecutive addition of unlabelled SNARE complex with the following final concentrations: $80 \mathrm{nM}(\sim 125 \mathrm{~s}), 160 \mathrm{nM}(\sim 225 \mathrm{~s})$, $240 \mathrm{nM}(\sim 310 \mathrm{~s}), 320 \mathrm{nM}(\sim 410 \mathrm{~s})$ ) to $\mathrm{Cpx}^{\mathrm{OG}}(80 \mathrm{nM})$ (figure $\left.4.12 \mathrm{c}\right)$. The curve exhibits a second increase in anisotropy upon addition of SNARE complex resulting in molar ratio of $1 / 2$. No further significant increase was observed for molar ratios of $1 / 3$ and $1 / 4$. Based on this experiment, a molar ratio of at least $1 / 1.5$ was used for the following experiments with SNARE complexes containing synthesised Synaptobrevin-2 derivatives.

Finally, it was tested, whether the Complexin binding reaction is still detectable with overall lower concentrations (figure $4.12 \mathrm{~d}$ ). Therefore, $\mathrm{Cpx}{ }^{\mathrm{OG}}(40 \mathrm{nM})$ was used and unlabelled SNARE complex (100 $\mathrm{nM}$ and $200 \mathrm{nM}$ ) was added to obtain ratios of $1 / 2.5$ and $1 / 5.0$. The increase in anisotropy observed was comparable to the experiments with $\mathrm{Cpx}{ }^{\mathrm{OG}}(80 \mathrm{nM})$, indicating that the overall concentration is not influencing the anisotropy signal (see chapter 4.4).

\subsubsection{Complexin Binding to SNARE Complexes Containing Syb-2 (28 - 84) Wild-Type (6)}

For SNARE complex formation of the synthesised Synaptobrevin-2 derivatives with the natural counterparts, purified $\Delta N$ complexes, consisting of Syntaxin-1A (183 - 162), SNAP-25 (no Cys mutant) and Synaptobrevin-2 $(49-96)$ were used (chapter 4.5.2 and 8.3.6). Due to retarded assembly properties of the adamantyl-modified Synaptobrevin-2 derivatives $\mathbf{7}$ and $\mathbf{1 0}$ (chapter 4.5.2), the incubation time for complex formation was extended to overnight at $4{ }^{\circ} \mathrm{C}$.

First of all, ternary SNARE complexes containing Syb-2 (28 - 84) wild-type (6) were obtained by overnight incubation with $\Delta \mathrm{N}$ complexes at $4{ }^{\circ} \mathrm{C}$ and are further referred to as $\Delta \mathrm{N} / \mathrm{Syb}-2$ wt (6). Complexin binding properties were investigated by fluorescence anisotropy by addition of $\Delta \mathrm{N} /$ Syb-2 wt (6) (200 nM) to $\mathrm{Cpx}^{\mathrm{OG}}$ (80 nM) (figure $4.13 \mathrm{~b}$, black curve). As expected, Complexin binds to the wild-type complex which is evident by anisotropy increase. Furthermore, two control experiments were conducted: 1) By combination of $\mathrm{Cpx}{ }^{\mathrm{OG}}$ and excess unlabelled $\mathrm{Cpx}(900 \mathrm{nM}$ ) prior to addition of the SNARE complex (figure $4.13 \mathrm{~b}$, red curve). No increase in anisotropy was observed due to the blockage of the binding site by unlabelled Cpx. 2) By addition of only $\Delta \mathrm{N}$ complex to $\mathrm{Cpx}^{\mathrm{OG}}$ (figure $4.13 \mathrm{~b}$, blue curve). Surprisingly, Complexin also 
a

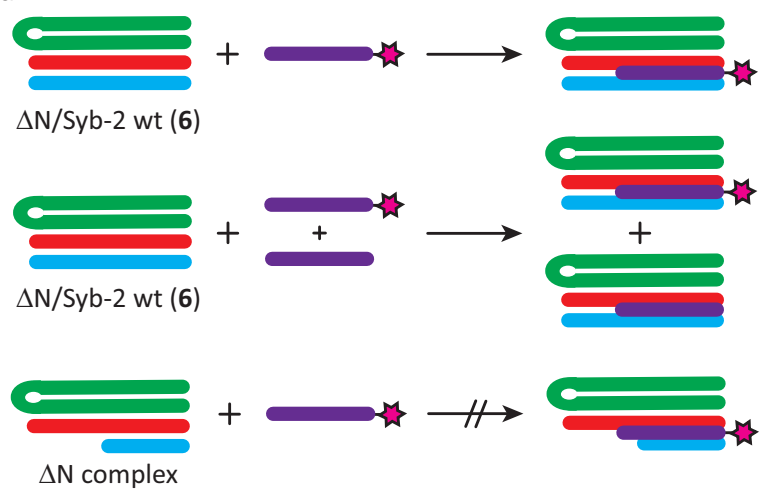

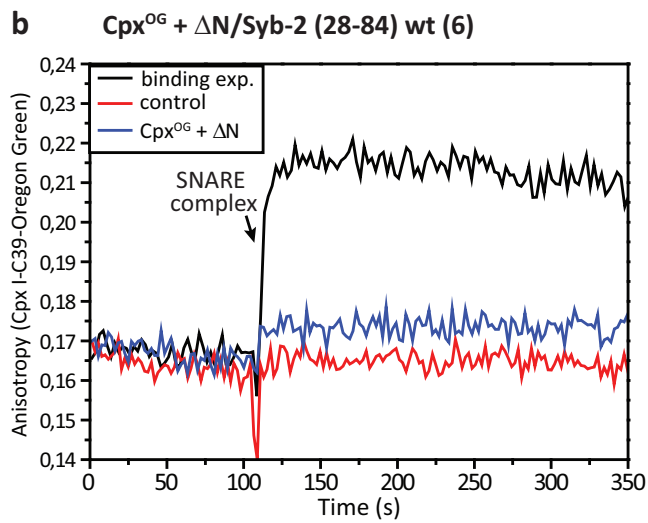

Figure 4.13: Complexin binding studies of SNARE complexes containing Syb-2 (28 - 84) wild-type (6), referred to as $\Delta \mathrm{N} / \mathrm{Syb}-2 \mathrm{wt}(\mathbf{6})$. (a) Scheme of performed binding reactions. (b) Fluorescence anisotropy experiments of Complexin binding (black curve) including control experiment with excess unlabelled Complexin (red curve). In addition, Complexin binding to the $\Delta \mathrm{N}$ complex was analysed (blue curve).

exhibits no binding to the $\Delta \mathrm{N}$ complex although the Syb-2 fragment $(49-96)$ comprises all interaction sites for Complexin (chapter 2.7.1). As a consequence, ternary SNARE complexes formed using $\Delta \mathrm{N}$ complexes are suitable for Complexin binding studies.

\subsubsection{Complexin Binding to SNARE Complexes Containing Adamantyl-Modified Synaptobrevin-2 Analogues}

SNARE complex formation including Syb-2 (28 - 84) E55N(Ad), D65N(Ad) (7) was obtained by incubation overnight at $4{ }^{\circ} \mathrm{C}$ with the same $\Delta \mathrm{N}$ complex as for Syb-2 (28-84) wild-type (6) (chapter 4.6.2). The derived SNARE complexes are further referred to as $\Delta N /$ Syb-2 $(55 / 65,7)$. Complexin binding properties were analysed by fluorescence anisotropy by addition of $\Delta N /$ Syb2(55/65, 7) (200 nM) to $\mathrm{Cpx}^{\mathrm{OG}}$ (80 nM) (figure 4.14 b, black curve). Surprisingly, Complexin is binding to the SNARE complex containing Syb-2 (28 - 84) E55N(Ad), D65N(Ad) (7) despite of the modification of the direct interaction site D65 of Synaptobrevin-2 by a hydrophobic and sterically demanding adamantyl moiety. In addition, a control experiment was performed with an excess unlabelled Cpx (900 nM) (figure $4.14 \mathrm{~b}$, red curve) and indicated that the increase in anisotropy in the first experiment is derived from $\mathrm{Cpx}{ }^{\mathrm{OG}}$ binding to the complex.

Furthermore, Complexin binding to SNARE complexes including Syb-2 (28 - 84) Q58N(Ad) (10) was investigated. SNARE complex formation was initially performed by overnight incubation at $4{ }^{\circ} \mathrm{C}$ with the same $\Delta \mathrm{N}$ complex as for Syb-2 (28 - 84) wild-type (6) (chapter 4.6.2). The possibly derived SNARE complexes are further referred to as $\Delta \mathrm{N} / \mathrm{Syb}-2(58,10)$. Complexin binding properties were analysed by fluorescence anisotropy with an addition of $\Delta N /$ Syb$2(58,10)(200 \mathrm{nM})$ to $\mathrm{Cpx}^{\mathrm{OG}}(80 \mathrm{nM})$ (figure $4.15 \mathrm{~b}$, black curve). Indeed, no increase in anisotropy was observed for the binding reaction of Complexin to the $\Delta N / \operatorname{Syb}-2(58,10)$ 
a

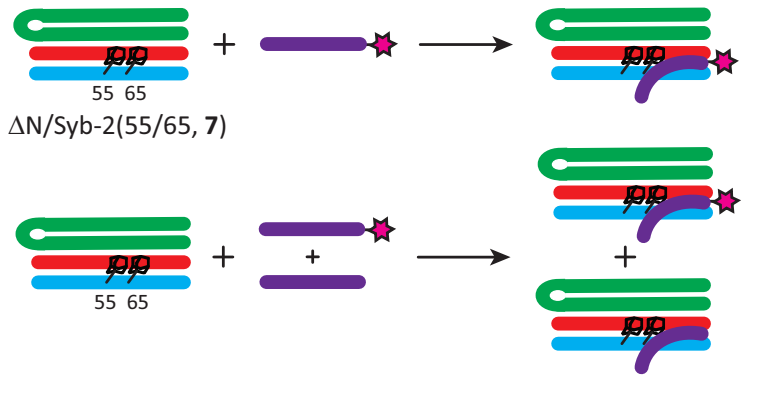

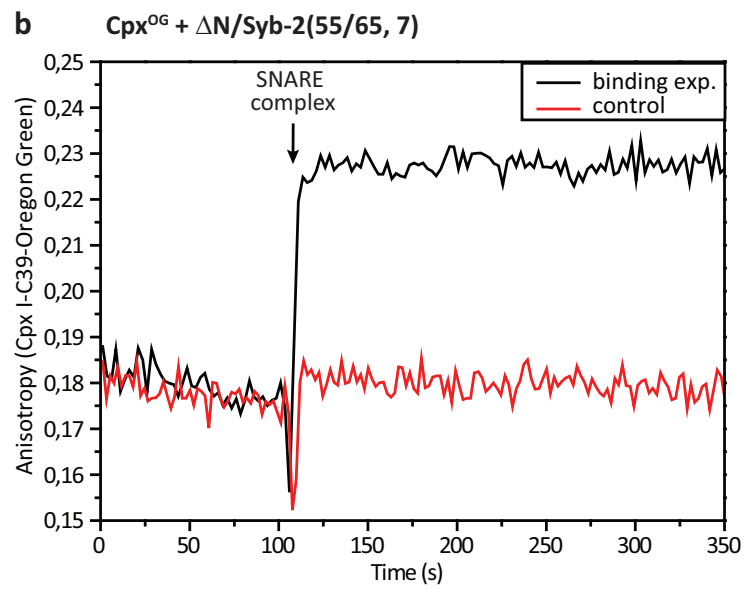

Figure 4.14: Complexin binding studies of SNARE complexes containing Syb-2 (28 - 84) E55N(Ad), D65N(Ad) (7), referred to as $\Delta N /$ Syb-2 $(55 / 65,7)$. (a) Scheme of performed binding reactions. (b) Fluorescence anisotropy experiments of Complexin binding (black curve) and control experiment including an excess of unlabelled Complexin (red curve).

complex as well as no signal was detected for the control experiment with excess of unlabelled Cpx (figure $4.15 \mathrm{~b}$, red curve). Complexin exhibits no binding affinity to individual SNARE proteins or binary SNARE complexes consisting of two Syntaxin-1A helices and SNAP-25, 72, 73 , In such a case, it has to be validated that the ternary SNARE complex was formed and that the absence of the signal is not derived from unsuccessful complex formation. For this purpose, labelled and unlabelled complexes were formed, using OG-labelled $\Delta N$ complex $\left(\Delta N^{O G}\right)$ and unlabelled $\Delta \mathrm{N}$ complex as described in chapter 4.5 .2 by overnight incubation at $4{ }^{\circ} \mathrm{C}$. The comparison of anisotropy levels between $\Delta \mathrm{N}^{\mathrm{OG}}$ and the probes $\Delta \mathrm{N} /$ Syb-2wt $(\mathbf{6})$ and $\Delta \mathrm{N} /$ Syb$2(58,10)$ provides a rough estimation of the quality of complex formation (figure $4.15 \mathrm{~b}$, inset). By displacement of the labelled Syb-2 fragment from the $\Delta \mathrm{N}$ complex, the anisotropy decreased due to lower molecular weight of dissociated Syb-2 fragment (cf. chapter 4.4). Hence, successful complex formation and displacement should exhibit a significantly lower anisotropy level then the $\Delta N^{O G}$ before. A comparison of anisotropy levels of $\Delta N^{O G}$ (figure 4.15 inset, black curve) and $\Delta \mathrm{N} / \mathrm{Syb}-2$ wt (6) (figure 4.15 inset, red curve) shows that the successfully assembled complex $\Delta \mathrm{N} /$ Syb-2 wt $(\mathbf{6})$ exhibited a significantly lower anisotropy level $(\sim 0.16)$ then the $\Delta \mathrm{N}^{\mathrm{OG}}$ complex $(\sim 0.25)$. Additionally, in this case the complex formation was already proved due to successful Complexin binding to $\Delta \mathrm{N} /$ Syb-2 wt (6) (chapter 4.6.2). Unfortunately, in the case of $\Delta N /$ Syb-2 $(58,10)$ the anisotropy level was only insignificantly lower $(\sim 0.24)$ then for $\Delta \mathrm{N}^{\mathrm{OG}}(\sim 0.25)$ which resulted in the assumption of unsuccessful complex formation for $\Delta \mathrm{N} / \mathrm{Syb}-2(58,10)$ (figure 4.15 inset, black and blue curves). Consequently, no Complexin binding was observed in this case because Complexin did not bind to individual SNARE proteins or to the $\Delta \mathrm{N}$ complex.

Further investigations were conducted to optimise conditions for SNARE complex formation with Syb-2 (28 - 84) Q58N(Ad) (10). Eventually, the same conditions as used prior to disassembly experiments (chapter 4.5.3) were again successful and ternary SNARE complexes 

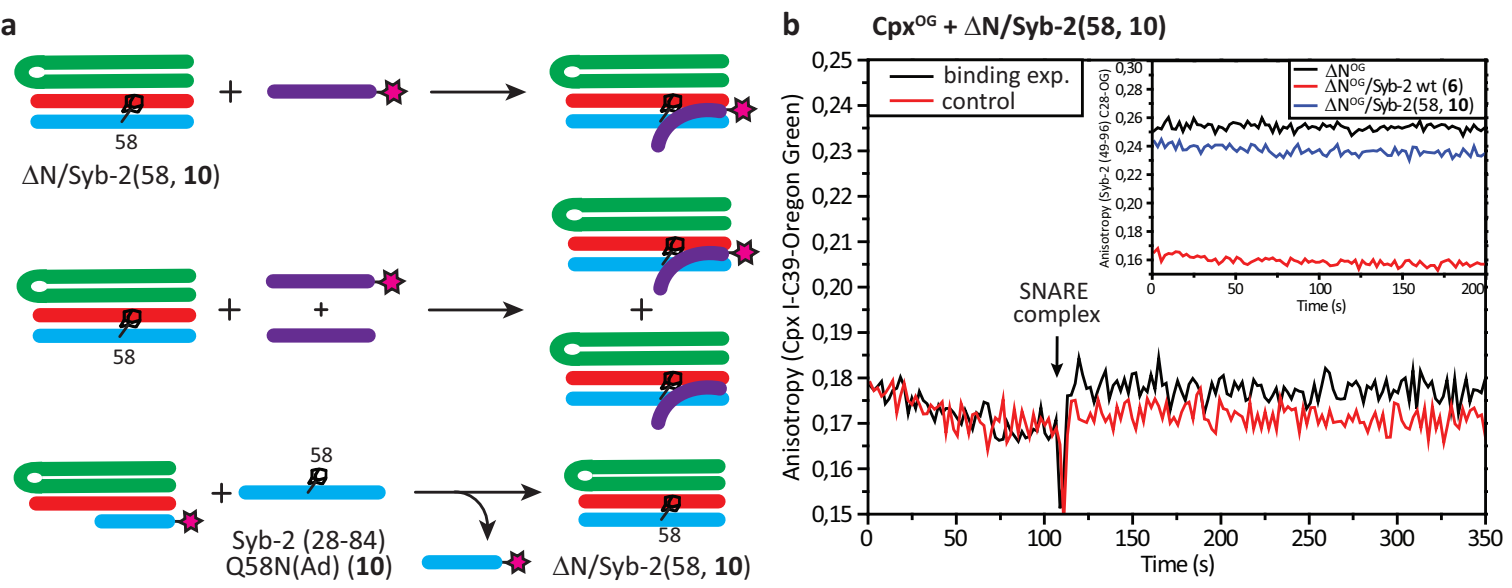

Figure 4.15: Complexin binding studies of SNARE complexes containing Syb-2 $(28-84)$ Q58N(Ad) (10), referred to as $\Delta \mathrm{N} /$ Syb-2(58, 10). (a) Scheme of performed binding reactions (top and middle) as well as ternary SNARE complex formation using labelled $\Delta \mathrm{N}$ complex (bottom). (b) Fluorescence anisotropy experiments of Complexin binding (black curve) and control experiment including an excess of unlabelled Complexin (red curve). (inset) Rough validation of ternary SNARE complex formation with labelled $\Delta \mathrm{N}$ complex $\left(\Delta \mathrm{N}^{\mathrm{OG}}\right)$.

containing Syb-2 (28 - 84) Q58N(Ad) (10) were obtained from monomers, further referred to as $\mathrm{SN} 25 / \operatorname{Syx} 1 \mathrm{~A} / \operatorname{Syb}(58,10)$. It turned out that usage of monomers, detergent-containing buffer (CHAPS) as well as Syntaxin-1A $(183-288)$ is crucial for complex assembly with this derivative. The experimental details are described in chapter 8.3.6 including conditions which were not successful. For better comparison, SNARE assembly with Syb-2 (28 - 84) wild-type (6) was also derived from monomers without detergents and Syx-1A-TMD. The properties of the formed complexes were investigated in parallel to SN25/Syx1A/Syb(58, 10) complexes. Wild-type complexes from monomers are further referred to as SN25/Syx1A/Sybwt (6).

Successful ternary complex formation was validated by SDS-PAGE using $12 \%$ SCHAEGGER gel and Coomassie Blue staining (figure $4.16 \mathrm{a}$ ), due to the fact that the ternary SNARE complex is SDS resistant. 27] An upper band ( 35 - $40 \mathrm{kDa})$ was observed for SN25/Syx1A/Sybwt (6) as well as for SN25/Syx1A/Syb $(58,10)$ which proved the presence of ternary SNARE complexes. Note that in the case of SN25/Syx1A/Sybwt (6) a diluted sample was used which resulted in overall lower band intensities. In addition, the complex band was slightly shifted to lower molecular weight compared to SN25/Syx1A/Syb $(58,10)$ due to Syntaxin-1A lacking the TMD.

Finally, Complexin binding to these two complexes was investigated by fluorescence anisotropy (figure 4.16 b). As expected, Complexin binds to the wild-type complex (SN25/Syx1A/Syb wt (6)) (figure $4.16 \mathrm{~b}$, black curve) but surprisingly, no increase in anisotropy was observed for the complex SN25/Syx1A/Syb(58, 10) (figure 4.16 b, red curve). This absence of anisotropy increase probably resulted from the influence of the Synaptobrevin-2 modification on Complexin binding due to the fact that the presence of ternary complex was already validated by SDS-PAGE 

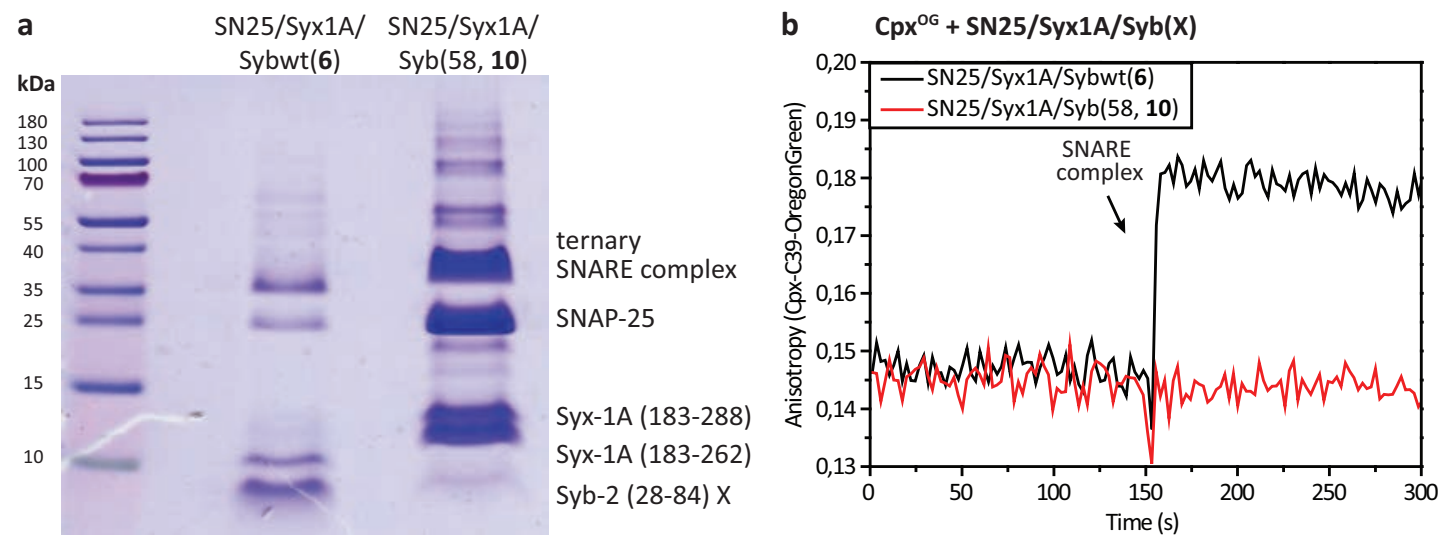

Figure 4.16: (a) Validation of complex formation of ternary SNARE complex from monomeric species containing Syb-2 (28 - 84) wild-type (6), referred to as SN25/Syx1A/Sybwt (6), and containing Syb-2 (28 - 84) Q58N(Ad) (10), referred to as SN25/Syx1A/Syb(58, 10), by SDSPAGE (12\% SchaEgGer gel stained with Coomassie blue). Note that SN25/Syx1A/Sybwt (6) $(<10 \mu \mathrm{L})$ was diluted with HEPES buffer $(30 \mu \mathrm{L})$ and mixed with sample buffer $(6 \mu \mathrm{L})$ prior to loading on the gel, in contrast to $\operatorname{SN} 25 / \operatorname{Syx} 1 \mathrm{~A} / \operatorname{Syb}(58,10)(24 \mu \mathrm{L})$ mixed with sample buffer $(6 \mu \mathrm{L})$. Hence, dilution is the reason for overall lower intensities of gel bands in the case of SN25/Syx1A/Sybwt (6). Samples were not boiled in sample buffer. (b) Complexin binding studies with SN25/Syx1A/Sybwt (6) (black curve) and SN25/Syx1A/Syb(58, 10) (red curve) by fluorescence anisotropy.

and possible non-binding to individual SNARE proteins can be excluded. The last possibility that the absent anisotropy increase was not based on the modification, was by formation of the binary complex, instead of ternary, consisting of SNAP-25 and two Syntaxin-1A helices. It was previously reported that Complexin did not bind to the binary complex. ${ }^{72}$.

To exclude this possibility, unlabelled and labelled binary complexes were formed by mixing SNAP-25/OG-labelled SNAP-25 and Syntaxin-1A in a molar ratio of 1/2 (chapter 8.3.6), incubated overnight at room temperature, and investigated by SDS-PAGE and fluorescence anisotropy (figure 4.17). As previously reported, the binary complex SN25/Syx1A was not SDSresistant and consequently exhibited no upper band at $\sim 40 \mathrm{kDA}$ (figure 4.17 a, lane 3), whereas the complexes SN25/Syx1A/Sybwt (6) and SN25/Syx1A/Syb(58, 10) without detergent (figure 4.17 a, lane 1 and 2) revealed upper bands, and consequently formation of SDS-resistant ternary complexes. Note that in this case for complex formation of $(\operatorname{SN} 25 / \operatorname{Syx} 1 \mathrm{~A} / \mathrm{Syb}(58, \mathbf{1 0})$ no detergents were used, and therefore, less ternary complexes were present than in the successful case with detergents (figure 4.16 a). In addition, binary complex formation was roughly validated by fluorescence anisotropy using OG-labelled SNAP-25 (SN25 OG) for labelled complexes as well as comparison of anisotropy levels (figure $4.17 \mathrm{~b}$, inset). In this case, upon complex formation the anisotropy level increases due to increasing molecular weight. Hence, formed binary complex should exhibit a higher anisotropy level than the free SN25 OG. Indeed, fluorescence anisotropy experiment revealed for $\mathrm{SN} 25^{\mathrm{OG}}$ a lower anisotropy level $(\sim 0.18)$ than for the probe after incubation with Syntaxin-1A $(\sim 0.22)$ which is a rough confirmation 
of a binary complex formation. Finally, Complexin binding properties to SN25/Syx1A were investigated by fluorescence anisotropy (figure 4.17 b). Addition of SN25/Syx1A (200 nM) to $\mathrm{Cpx}^{\mathrm{OG}}(80 \mathrm{nM})$ resulted in no increase in anisotropy which confirmed that Complexin did not bind to the binary complex due to the missing binding site between the Syntaxin- $1 \mathrm{~A}$ and Synaptbrevin-2 helices.
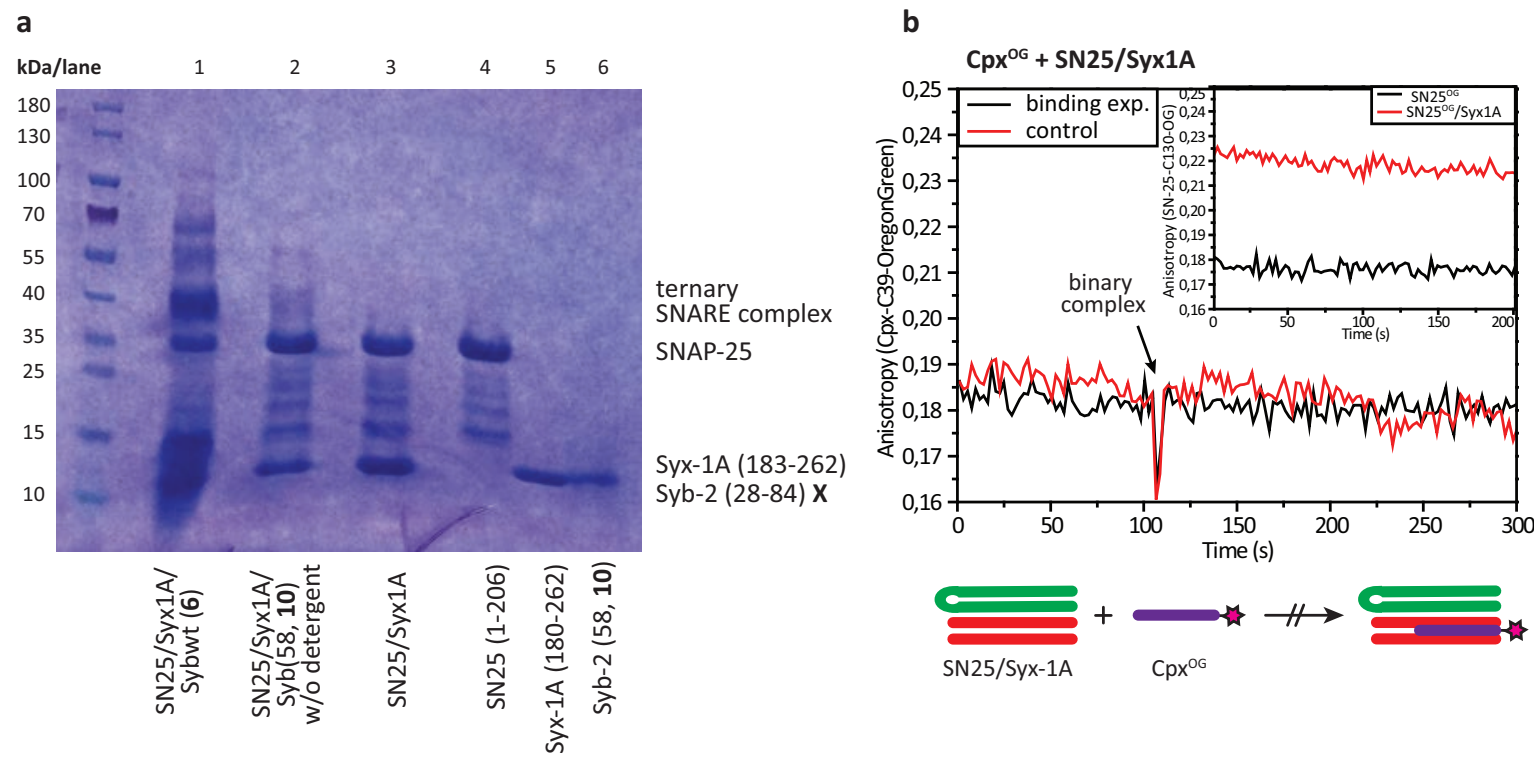

Figure 4.17: (a) Validation of complex formation of selected ternary and binary SNARE complexes by SDS-PAGE (pre-casted $4 \%-12 \%$ gradient gel stained with Coomassie blue). The binary complex SN25/Syx1A was not SDS-resistant and did not show an upper band on the SDS gel (lane 3). (b) Fluorescence anisotropy experiment of Complexin binding to binary complex SN25/Syx1A (black curve) and control experiment with excess unlabelled Complexin (red curve). (bottom) Scheme of performed Complexin binding reaction. (inset) Rough validation of the binary complex formation with labelled SNAP-25 (SN25 OG) by fluorescence anisotropy.

To conclude, non-binding of Complexin to SNARE complexes containing Syb-2 (28 - 84) Q58N(Ad) (10) had to base on the modification of position Q58 with admantyl-modified amino acid due to the following facts:

1. Successful ternary SNARE complex formation including Syb-2 (28 - 84) Q58N(Ad) (10) was confirmed by SDS-PAGE. An upper band ( $35-40 \mathrm{kDa})$ was observed which had to be the ternary complex due to the SDS-resistance (figure 4.16 a).

2. Possible non-binding resulting from the presence of only individual SNARE proteins can be excluded due to the upper band in the SDS gel.

3. Non-binding resulting from the presence of binary complex alone can also be excluded due to the fact that the binary complex was not SDS-resistant and did not exhibit an upper band in SDS gels (figure $4.17 \mathrm{a}$ ).

Consequently, Complexin did not bind to the SNARE complex containing Syb-2 (28 - 84) $\mathrm{Q} 58 \mathrm{~N}(\mathrm{Ad})$ (10) due to the adamantyl-modification. Surprisingly, the position of modification 
Q58N(Ad) was totally inhibiting Complexin binding although it was not found to be a direct interaction site and was only in close proximity to one (D57).

To summarise, Complexin binding experiments with SNARE complexes containing Syb-2 (28 - 84) wild-type (6), Syb-2 (28 - 84) E55N(Ad), D65N(Ad) (7), or Syb-2 (28 - 84) $\mathrm{Q} 58 \mathrm{~N}(\mathrm{Ad})$ (10) were performed and analysed by fluorescence anisotropy (figure 4.18). As expected, Complexin was binding to the wild-type SNARE complex, but surprisingly also to complex $\Delta \mathrm{N} /$ Syb-2(55/65, 7), although the direct interaction site D65 was modified with an adamantyl moiety. Interestingly, Complexin binding was totally inhibited by SNARE complexes containing Syb-2 (28 - 84) Q58N(Ad) (10). Complex formation with these derivatives emerged to be challenging but eventually the appropriate conditions could be determined. In addition, it was shown that Complexin did not bind to the binary complex SN25/Syx1A or to the $\Delta N$ complex.

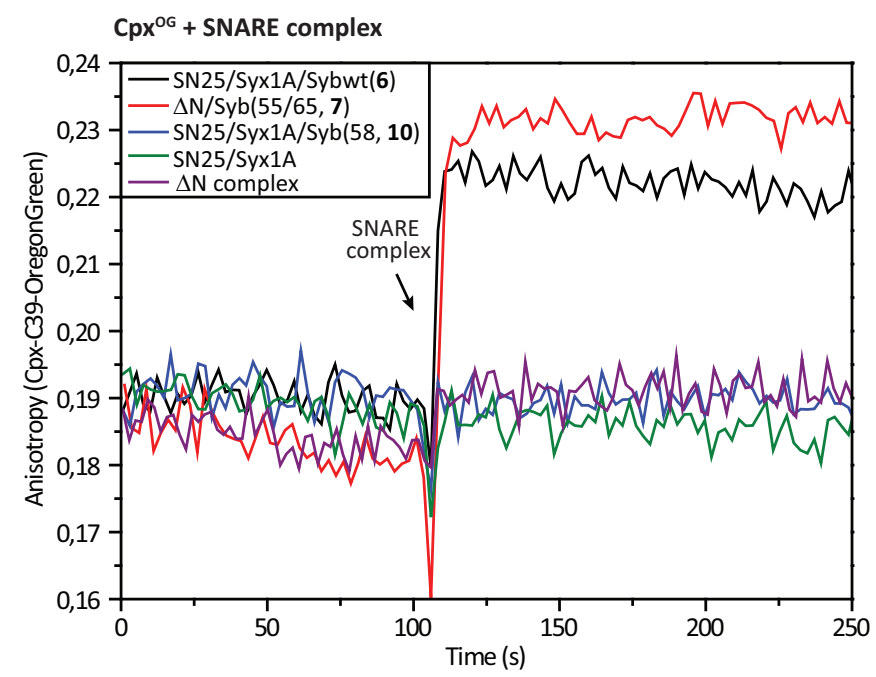

Figure 4.18: An overview of measured Complexin binding experiments with fluorescence anisotropy. Corresponding SNARE complexes (200 nM) were added to $\mathrm{Cpx}^{\mathrm{OG}}(80 \mathrm{nM})$. The addition is indicated by an arrow. 


\subsection{Conclusion}

The motivation of this study was to validate the properties of adamantyl-modified Synaptobrevin2 analogues designed by Dr. A. Groschner. ${ }^{119}$ The modification was introduced to develop an inhibitor for $\alpha$-SNAP/NSF-mediated disassembly reaction of SNARE complex, and thereby, acquire further insights into mechanism of this process. Based on identified interaction sites between $\alpha$-SNAP and Synaptobrevin-2, ${ }^{64]}$ suitable sequence positions were chosen for modification. A sterically demanding side chain on the surface of SNARE complex was thought to prevent or at least weaken $\alpha$-SNAP binding. The admantyl-modified amino acid building block Fmoc-Asn(Ad)-OH (4) ${ }^{[119}$ was synthesised for site-specific modification of Synaptobrevin2 SNARE motif by solid-phase peptide synthesis. A selection of five previously published Synaptobrevin-2 derivatives ${ }^{[119}$ was made based on their affinity to form ternary SNARE complexes and influences on disassembly reactions, to validate these properties by fluorescencebased methods. Beside the synthesis strategy with combined automated microwave-assisted and manual SPPS, the wild-type Synaptobrevin-2 analogue 6 was also successfully synthesised using only automated microwave-assisted SPPS. For this long peptide sequences (56 amino acids), purification by HPLC is still challenging. Further improvement in SPPS strategies are needed to decrease the time-consumption and increase the yields of the syntheses.

Ternary SNARE complexes with the natural counterparts were successfully formed for all investigated adamantyl-modified Synaptobrevin-2 derivatives. Especially for derivative Syb-2 $(28$ - 84) Q58N(Ad) (10), complex formation emerged to be challenging, and the appropriate conditions had to be found. It proved necessary to use detergent-containing buffer (CHAPS, $1 \%$ ) and Syntaxin-1A (183 - 288) including the TMD (chapter 4.6.3 and 8.3.6).

To summarise, SNARE assembly properties were investigated with OG-labelled, stabilized acceptor complexes ( $\Delta \mathrm{N}$ complex) consisting of SNAP-25 $(1-206)$ no Cys mutant, Syntaxin-1A (183 - 262), and Synaptobrevin-2 (49 - 96) C79-OG. Upon addition of longer Synaptobrevin-2 sequences, the small fragment will be displaced and ternary SNARE complex is formed rapidly. With OG-labelled Syb-2 fragment, the displacement can be measured by fluorescence anisotropy and complex formation can be indirectly monitored. 11] Investigation of SNARE assembly properties of Syb-2 (28 - 84) wild-type (6) showed that this derivative is comparable to the normally used soluble Syb-2 $(1-96)$. On the other hand, assembly properties of Syb-2 $(28-84)$ E55N(Ad), D65N(Ad) (7) and Syb-2 (28 - 84) Q58N(Ad) (10) showed clear retardation of complex formation due to the modifications. The adamantyl modifications in these derivatives were close to the $N$-terminal end of Syb-2 fragment $(49-96)$ and may disturb the displacement process. Probably, SNARE complex formation using monomers would not be impaired in this extend.

The formed ternary SNARE complexes containing adamantyl-modified Syb-2 derivatives were 
successfully reconstituted into SUVs by co-micellisation method. Reconstitution was necessary prior to disassembly experiments, due to the membrane-dependent efficiency of $\alpha$-SNAP. [59] By co-floatation assay, successful reconstitution and complex formation was confirmed as well as rough purification of liposomes was obtained.

Reconstituted SNARE complexes containing Syb-2 derivatives were investigated regarding their ability to impair $\alpha$-SNAP binding, and consequently, disassembly reaction mediated by NSF/ATP. Due to OG-labelled SNAP-25 used for complex formation, $\alpha$-SNAP binding was detected by an increase in fluorescence anisotropy, whereas the complex disassembly was monitored by a decrease in anisotropy. Unfortunately, $\alpha$-SNAP was able to bind to all investigated SNARE complexes containing adamantyl modification. Especially, Syb-2 (28 - 84) Q58N(Ad) (10) had no effect on $\alpha$-SNAP binding as well as disassembly reaction which was previously found to be the most promising derivative for inhibitor development. 119] Possible explanations for these results are: Firstly, even if one binding site for $\alpha$-SNAP is blocked due to the modifications, the SNARE complex could be disassembled by NSF with less than three or four $\alpha$-SNAPs, like it was shown for the V7-20 S complex. [50 53] Secondly, the modified positions in the Synaptobrevin-2 sequence based on a 3/1 stoichiometry of $\alpha$-SNAP/SNARE complex, ${ }^{64}$ and were thought to cover binding sites of two $\alpha$-SNAPs. Probably, with four $\alpha$-SNAPs binding around the SNARE complex, the modifications may cover only the binding site of one $\alpha$-SNAP. Absence of one $\alpha$-SNAP may be overcome easily by the system.

Previously, investigations of disassembly properties were performed by time-dependent SDSPAGE which had a rough time-resolution of $\sim 5 \mathrm{~min}$. SNARE disassembly reaction was detected in the range of $10-15 \mathrm{~min}$. $\frac{119}{2}$ With this currently performed fluorescence anisotropy measurements, time-resolution was in the range of seconds and fast binding of $\alpha$-SNAP as well as fast disassembly by NSF within seconds was observed. Using stopped-flow and higher amounts of synthesised Synaptobrevin-2 derivatives, rapid kinetics could be measured with these methods. Gel band intensities were previously used for quantitatively visualising the influence of modifications on the disassembly reaction. ${ }^{[119]}$ With fluorescence anisotropy clear determination of $\alpha$-SNAP binding and disassembly of SNARE complexes was made, due to distinct and fast increase and decrease in anisotropy, respectively. In addition, fluorescence anisotropy is not as perturbed by minor changes in concentrations as gel band intensities are. In conclusion, the results obtained by fluorescence anisotropy are more significant than by SDS-PAGE and adamantyl modifications had no effect on $\alpha$-SNAP binding.

Due to suggested competitive binding of $\alpha$-SNAP and Complexin to the SNARE complex surface, some adamantyl-modified Synaptobrevin-2 derivatives were investigated regarding Complexin binding. Using OG-labelled Complexin, binding event was successfully monitored upon addition of corresponding SNARE complexes by an increase in fluorescence anisotropy. As expected, Complexin was binding to SNARE complexes containing Syb-2 (28 - 84) wild-type (6), but also to complexes containing Syb-2 (28 - 84) E55N(Ad), D65N(Ad) (7). The latter 
result is surprising, due to the fact that a direct interaction site with Complexin (D65) is modified by a sterically demanding and hydrophobic adamantyl moiety. Probably, the interaction with the neighbouring residues D64 and D68 was able to overcome this disturbance. It would be interesting to investigate the binding kinetics of Complexin to this modified SNARE complex due to the fact that already the insertion of alanine mutations (Syb-2 D64A, D65A, D68A) resulted in ten-fold lower binding affinities of Complexin. ${ }^{80}$ 82] Additionally, these residues were proposed to be the conformational switch region for Complexin to change its accessory $\alpha$-helix from open to closed state. ${ }^{80}$ [2] Investigation of $\mathrm{D} 65 \mathrm{~N}(\mathrm{Ad})$ regarding this issue, especially regarding the binding kinetics, would be interesting.

More importantly, SNARE complexes containing Syb-2 (28 - 84) Q58N(Ad) (10) were totally inhibiting Complexin binding, although the position of modification was no direct interaction site and was only in close proximity to one (D57). This derivative exhibited the most problems with complex formation which might lead to the hypothesis that the position Q58 is crucial for SNARE zippering, Complexin binding, and might be energetically critical. Previously, it was suggested as the most promising derivative for $\alpha$-SNAP inhibition, ${ }^{119]}$ which was now found not to be the case, but definitely this modification had an impact on SNARE complex properties. 


\section{Synthesis and Impact on SNARE Zippering of a Soluble Caged Synaptobrevin-2 Analogue}

The aim of designing a soluble caged Synaptobrevin-2 derivative was to obtain a Synaptobrevin2 inactive towards SNARE assembly in the caged state, and upon UV irradiation, reactivation towards SNARE complex formation. In vesicle fusion assays in vitro, the soluble Synaptobrevin-2 $(1-96)$ is used as a competitive inhibitor. It competes with the Synaptobrevin-2 reconstituted into liposomes for the binding site of the Q-SNARE complex and thereby inhibits vesicle fusion. It is often used as a control experiment to confirm that the measured vesicle fusion is derived from SNARE complex assembly (e.g. PARK et al. ${ }^{[60]}$ ). In addition, resistance against the inhibitory function indicates presence of already zippered complexes.

For in vivo studies, however, injection of suitable inhibitors needed long incubation times of 10 - 30 min before inhibition was detectable in chromaffin cells ${ }^{[130]}$, probably due to slow diffusion of the molecules. In the case of soluble Synaptobrevin-2, inhibition and indirect testing for acceptor complexes needed to occur fast due to the presence of NSF/ $\alpha$-SNAP in vivo which would quickly dissociate inhibited complexes again.

With a caged version of soluble Synaptobrevin-2, an inactive inhibitor can be injected and preincubated in the cells, and upon irradiation, a jump in concentration of the active inhibitor enables an immediate detection of acceptor complexes before disassembly. In addition, a caged Synaptobrevin-2 derivative which arrests the SNARE complex formation in the middle of the SNARE motifs, and upon irradiation, zippering is proceeded, gives novel opportunities to study the process of SNARE zippering. Furthermore, the action of executive and regulatory proteins like Munc18s, Complexins or Synaptotagmins on arrested SNARE complexes can be investigated.

One successful example for caging was initially developed by NEHER \& ZUCKER [113 114] in 1993, and in the meantime, the application of caged $\mathrm{Ca}^{2+}$ was well-established for studying exocytosis in vivo. It provides the advantage of mimicking the jump of $\mathrm{Ca}^{2+}$ concentration as it would occur upon $\mathrm{Ca}^{2+}$ influx into a nerve terminal. ${ }^{115}$ More recently, caged phosphatidylinositol 4,5-bisphosphate $\left(\mathrm{PIP}_{2}\right)$ was developed by SøRENSEN et al. for investigation of the interaction with Synaptotagmin-1 (unpublished results). 
To design a caged soluble Synaptobrevin-2, the following points had to be considered: First of all, suitable amino acid residues had to be identified for modification with photocleavable protecting groups. The caged version should prevent complete zippering but ideally exhibit a partially zippered state. Available studies of Synaptobrevin-2 mutants were investigated in chapter 5.1 regarding this issue. Second, photoclevable protecting groups can only be introduced at limited positions. Possible amino acid side chains for a modification are: Asp, Arg, Cys, Glu, Lys, Ser, Thr, and Tyr. Hence, hydrophobic amino acid residues, mainly contributing to SNARE complex assembly, are not suitable for attachment of caging groups. Third, a photoremovable protecting group had to be chosen providing straightforward synthesis of the corresponding amino acid building block and compatibility with Fmoc-SPPS. Lastly, the caging groups had to provide fast photocleavage or so-called uncaging properties and UV absorption $\lambda>345 \mathrm{~nm}$ to be suitable for possible in vivo application.

In chapter 5.2, the final design and synthesis strategy for soluble caged Synaptobrevin-2 will be described as well as the synthesis strategies for the used photocleavable amino acid building blocks (chapter 5.2.1). After successful synthesis, photocleavage properties were analysed (chapter 5.2.2 and 5.2.3) and preliminary binding studies regarding SNARE complex assembly were performed and analysed by fluorescence anisotropy (chapter 5.3).

\subsection{SNARE Zippering Studied by Synaptobrevin-2 Mutants}

A variety of SNARE zippering studies with Synaptobrevin-2 mutants were performed using truncated sequences ${ }^{11}[12,19]$ as well as point mutations ${ }^{[14-18]}$. The former mutants are shown in figure 5.1 and the latter are summarized in table 5.2 .

The zippering studies including truncated Synaptobrevin-2 sequences early revealed the problem of binary complex formation consisting of Syntaxin-1A/SNAP-25 (2/1). This finding resulted in development of the stabilized acceptor complex, the so-called $\Delta \mathrm{N}$ complex, including the Syb-2 $(49-96)$ fragment. 11] Zippering processes of various truncated Syb-2 sequences were indirectly investigated by monitoring labelled Syb-2 $(49$ - 96) fragment displacement (cf. chapter 4.5.2). The first study by POBBATI et al. ${ }^{11}$ indicated a $C$-terminal overlap with the fragment was not sufficient but the $N$-terminal zippering was necessary for displacement (figure 5.1 a). The fastest displacement was observed for the whole cytosolic part of Syb-2 $(1-96)$. In addition, it was shown that two non-overlapping fragments bind simultaneously (Syb-2 (1 - 59) and Syb-2 $(60-96))$. 11] A following study by WIEDERHOLD \& FASSHAUER ${ }^{[12]}$ additionally revealed that, besides $N$-terminal zippering, the overlapping region needed to comprise several helix turns for displacement. Syb-2 $(1-52)$ did not exhibit fragment displacement but was shown 

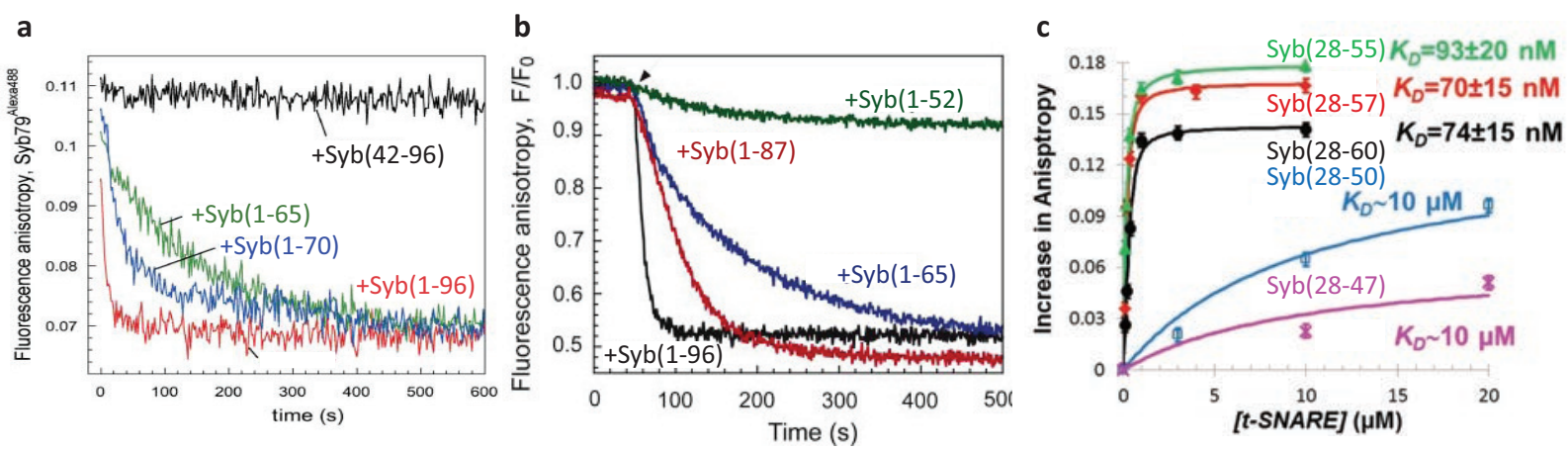

Figure 5.1: Overview of investigated Syb-2 truncated sequences regarding SNARE assembly properties. (a) Syb-2 (42 - 96), Syb-2 (1 - 65), Syb-2 (1 - 70), and Syb-2 $(1-96)$ investigated by fluorescence anisotropy regarding labelled Syb-2 $(49-96)$ fragment displacement of $\Delta N$ complex. Figure used from PoBBati et al.11] From reference [11]. Reprinted with permission from AAAS. (b) Syb-2 (1 - 52), Syb-2 (1 - 65), Syb-2 (1 - 87), and Syb-2 $(1-96)$ investigated by fluorescence anisotropy regarding labelled Syb-2 (49-96) fragment displacement of $\Delta \mathrm{N}$ complex. Figure used from Wiederhold \& Fasshauer. ${ }^{12]}$ (c) Syb-2 $(28-47)$, Syb-2 (28 - 50), Syb-2 $(28-55)$, Syb-2 $(28-57)$, and Syb-2 $(28-60)$ investigated by fluorescence anisotropy regarding concentration-dependent binding to Syntaxin-1A and SNAP-25. Figure modified from LI et al.19] This is an unofficial adaptation of an figure that appeared in an ACS publication. ACS has not endorsed the content of this adaptation or the context of its use.

to bind to the $\Delta \mathrm{N}$ complex (figure 5.1 b). The comparison of SNARE complexes containing Syb-2 (1 - 52)/Syb-2 (49 - 96) and Syb-2 (1 - 59)/Syb-2 (60 - 96), respectively, revealed a higher stability in the longer $C$-terminal portion for the former resulting in easier dissociation of the $N$-terminal part. In the second case, the $C$-terminal Syb fragment was able to dissociate reversibly. In contrast, binding of Syb-2 $(71-96)$ to a so-called $\Delta C$ complex containing Syb-2 $(1-70)$ exhibited only low affinity analysed by ITC indicating that the $N$-terminal zippering plays a more important role than the $C$-terminal part. 12.

A third study by Li et al. ${ }^{[19]}$ investigated the binding properties of $N$-terminal Syb-2 fragments to Syntaxin-1A and SNAP-25 (figure 5.1 c). A dramatic reduction in binding affinity was observed for Syb-2 (28 - 47) and Syb-2 (28 - 50), whereas longer fragments exhibited higher affinities. The authors suggested a crucial function of the region around layer -1 (residue 53) for SNARE zippering. In addition, $N$-terminal Syb-2 fragments pre-bound to Syntaxin1A/SNAP-25 and reconstituted into liposomes were investigated regarding their activation properties in liposome fusion assays with liposomes containing $C$-terminal Syb- 2 fragments. In more detail, Syb-2 $(60-116)$ was reconstituted into liposomes and mixed with liposomes containing Syntaxin-1A/SNAP-25 with or without pre-bound $N$-terminal Syb-2 fragments. Without pre-bound Syb-2 fragment, no fusion was observed, indicating that the $N$-terminal zippering is necessary to trigger the $C$-terminal assembly and enable fusion. With pre-bound Syb-2 $(28-55)$ or Syb-2 $(28-57)$ activation of the complex for fusion was observed, whereas Syb-2 (28 - 44), Syb-2 (28 - 47), or Syb-2 (28 - 50) did not exhibit any activation. These results suggested that the $N$-terminal zippering needed to reach approximately layer -1 to enable the whole SNARE complex machinery for fusion. Hence, the middle layers represent a 
crucial part for SNARE complex assembly. ${ }^{19}$

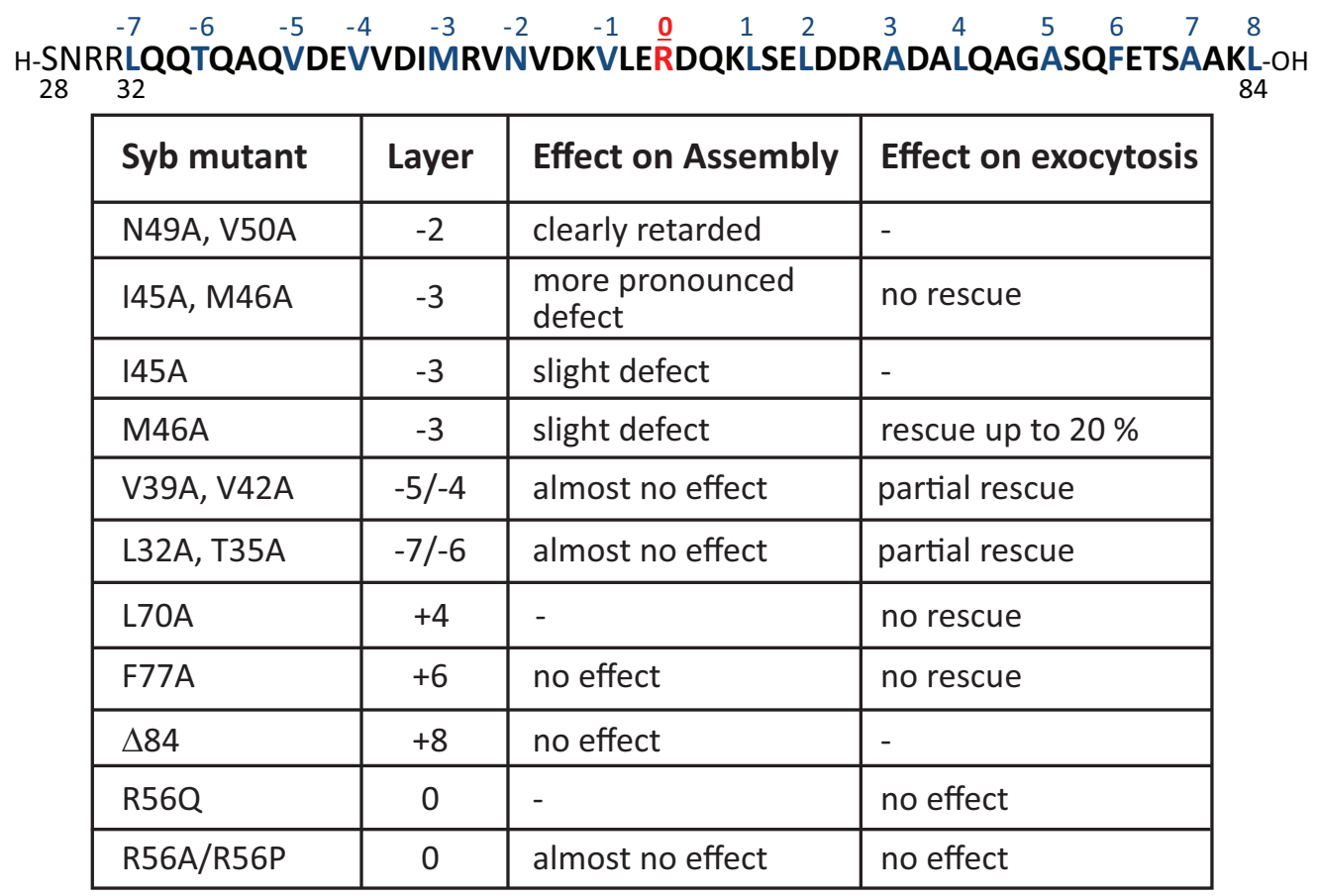

Table 5.2: Summary of Synaptobrevin-2 point mutation studies regarding SNARE complex formation and effect on exocytosis. SNARE assembly properties of Syb-2 $(1-96)$ N49A, V50A, Syb-2 (1 - 96) I45A, M46A, Syb-2 (1 - 96) I45A, and Syb-2 $(1-96)$ M46A [17] as well as Syb-2 (1 - 96) V39A, V42A, Syb-2 (1 - 96) L32A, T35A, Syb-2 (1 - 96) L70A, Syb-2 (1 - 96) F77A $[16]$ and Syb-2 $(1-96) \Delta 84^{[18}$ were investigated by fluorescence anisotropy using $\Delta N$ complex including labelled Synaptobrevin-2 (49-96) fragment. In addition, several mutants were investigated in vivo by rescue experiments using double knockout (Syb-2/Cellubrevin) mice chromaffin cells. The most pronounced effect showed double mutation of layer -3 (Syb-2 $(1-96)$ I45A, M46A). 17. The effect of Synaptobrevin-2 (1 - 116) R56Q on exocytosis was investigated using rescue experiments in cultured Syb-deficient neurons and exhibited comparable rescue properties as the wild-type. ${ }^{15}$ Investigations of Synaptobrevin-2 $(1-116)$ R56A and Synaptobrevin-2 $(1-116)$ R56P showed almost no effect on SNARE assembly in vitro analysed by fluorimetry as well as in vivo analysed by immunoprecipitation of cell lysates from HeLa cells. 14]

In most of the point mutation studies, alanine mutants were investigated due to alanine's small and hydrophobic side chain preventing strong impairments of the ternary SNARE complex stability. ${ }^{17}$ Especially, alanine mutations in layer -2 and -3 of the Synaptobrevin-2 SNARE motif were investigated due to highly conserved residues among R-SNAREs. Indeed, these mutants exhibited disturbed SNARE complex assembly analysed by fluorescence anisotropy using $\Delta \mathrm{N}$ complexes containing labelled Syb-2 $(49-96)$ fragment. Specifically, Syb-2 (1 - 96) 145A, M46A showed the most pronounced defect in Syb-2 fragment displacement of all tested mutants as well as no rescue of neurotransmitter release by overexpression in double knockout (Syb-2/Cellubrevin) mice chromaffin cells. In addition, the double mutant Syb-2 (1 - 96) N49A, V50A exhibited also a clearly retarded Syb-2 fragment displacement which both indicated a so-called "trigger site" for coiled coil formation between layer -2 and -4 . 17. These results were 
at least partially consistent with the truncated sequences suggesting layer -1 to be critical. 19. In contrast, double mutations of layer $-5 /-4$ (Syb-2 (1 - 96) V39A, V42A) and layer $-7 /-6$ (Syb-2 (1 - 96) L32A, T35A) exhibited almost comparable SNARE assembly properties as the wild-type and also partially rescued neurotransmitter release in double knockout chromaffin cells. Interestingly, single mutants of layer +4 (Syb-2 $(1-116)$ L70A) and layer +6 (Syb-2 $(1-116)$ F77A) exhibited both dramatic effects on exocytosis in chromaffin cells but with the latter comparable SNARE assembly properties like the wild-type. ${ }^{16}$

The deletion mutant of layer $+8($ Syb-2 $(1-116) \Delta 84)$ showed the same complex formation properties as wild-type Syb-2 but was found to prevent fusion of large liposomes in vitro and arrested them in a docked state. Hence, complete zippering of the SNARE motifs was possible with this mutant but to induce fusion further zippering of the linker and transmembrane regions was concluded to be necessary $[18$ ]

Surprisingly, mutations of the highly conserved zero layer exhibited almost no effect in vitro as well as in vivo. 14 15]

To summarize, modifications with photocleavable protecting groups should be localised in the middle of the SNARE motif from layer -1 to +1 to impair SNARE zippering. Especially, layer -1 was shown to exhibit a critical function. Regarding the pronounced effect of layer -3 double mutant Syb-2 I45A, M46A on SNARE assembly ${ }^{[17}$, these positions would presumably perfectly impair SNARE zippering, but unfortunately lle and Met are not suitable for caging group attachment. The same was true for the double mutant of layer -2 (Syb-2 N49A, V50A) 17 with Asn possible accessible for caging but Val not. Single modification of Asn would probably reduce impairment of SNARE zippering. Consequently, residues directly participating in the layer formation of SNARE complex cannot be modified with photocleavable protecting groups due to their hydrophobic side chains. The only exception comprises the hydrophobic zero layer. Hence, neighbouring residues contributing to coiled coil formation by electrostatic interactions need to be addressed for modification.

\subsection{Design and Synthesis of Soluble Caged Synaptobrevin-2}

The synthesis of the whole cytosolic part of Synaptobrevin-2 $(1-96)$ is too long for SPPS. Consequently, it was focused on synthesis of the SNARE motif $(28-84)$. If necessary, elongation could be performed by suitable ligation strategies. According to the Synaptobrevin-2 mutation studies described in the previous chapter, three possible regions within the Synaptobrevin-2 SNARE motif might be suitable for modification with caging groups to prevent or at least impair SNARE zippering: layer $-2 /-3$, layer -1 to +1 , or layer +6 . Due to the fact that the hydrophobic residues directly participating in layer formation are not available for caging (except for the 
hydrophilic zero layer), the coiled coil sequence was analysed by a helical-wheel (scheme 5.3).

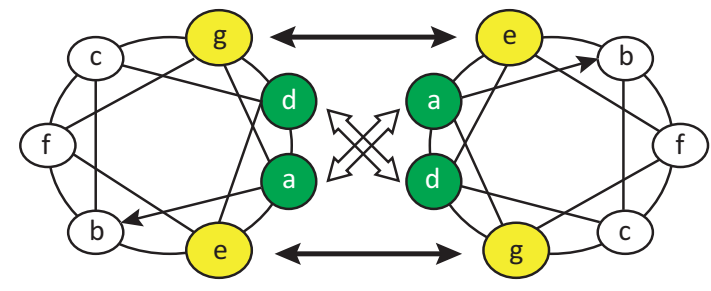

Scheme 5.3: Helical-wheel of a parallel coiled coil dimer including a heptad repeat of amino acids labelled from a to $g$. Main interactions are represented by the hydrophobic a/d interfaces (green) and the hydrophilic e/g interface (yellow). Scheme modified from RobSen MARSDEN \& Kros.131] (C) 2010 Wiley-VCH Verlag GmbH \& Co. KGaA, Weinheim.

The main interaction interfaces in a parallel coiled coil dimer are represented, which can also be transferred to a parallel tetramere as it is the case for the ternary SNARE complex. The hydrophobic a/d interfaces form the core of the coiled coil, whereas mainly charged residues contribute to the e/g interface by electrostatic interactions. Exactly the latter residues are accessible for modification with photocleavable protecting groups and represent a possible approach for soluble caged Synaptobrevin-2. Based on the mutation studies and the helical-wheel analysis, possible modification positions are summarized and categorised in three regions in scheme 5.4. Region I. comprises layer $-3 /-2$ which exhibited most pronounced effects in the alanine mutation studies. Nevertheless, in this region no suitable position for caging was found. Modification of M46 to a caged Cys residue and N49 to a caged Asp would be a compromise but the M46A single mutant exhibited only a slight defect in SNARE assembly experiments. Additional mutation of directly neighbouring residues was shown to be crucial within these layers but is not possible with photocleavable protecting groups. Region III. comprises layer $+6 /+7$ and three possible modifcation positions: F77, E78, and S80. The single mutant F77A exhibited no effect on SNARE assembly but dramatic reduction in exocytosis which indicated normal SNARE zippering until layer +6 but impairments in mediating fusion. Hence, these positions are not suitable to obtain a soluble caged Synaptobrevin-2 exhibiting partial SNARE zippering but would arrest SNARE complexes in a docked state. Lastly, region II. comprises layer -1 to +1 and is located approximately in the middle of the SNARE motif. Possible modification positions are: K52, R56, D57, and K59. Including the hydrophilic zero layer, this region includes four residues for photocleavable protecting groups and will be used for synthesis of a soluble caged Synaptobrevin-2 (28 - 84) K52K*, R56R*, D57D*, K59K* derivative.

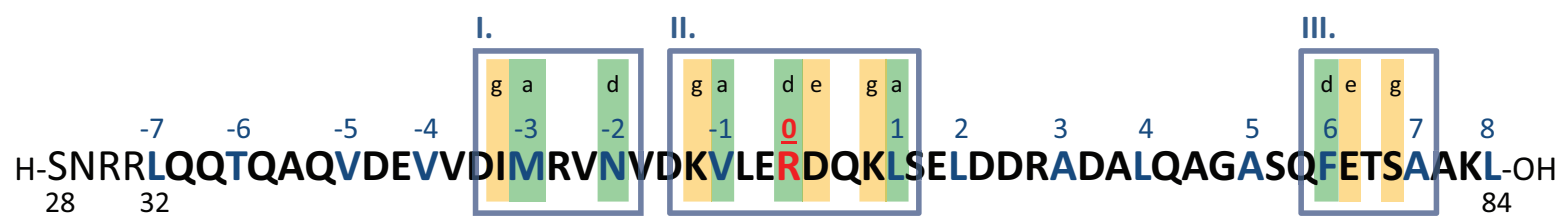

Scheme 5.4: Suitable regions of amino acid residues for modification of Synaptobrevin-2 (28 84) SNARE motif. Modification positions are categorised into three regions (I., II., III.) and coiled coil residues are labelled according to their contribution to the a/d (green) or e/g (yellow) interface. The hydrophobic layers contributing to SNARE complex coiled coil are highlighted and labelled in blue. 
For synthesis of the caged Syb-2 derivative, amino acid building blocks bearing photocleavable protecting groups at the side chains suitable for Fmoc-SPPS had to be synthesised (chapter 5.2.1). To test the properties of these building blocks, short test peptide sequences were synthesised and analysed regarding synthesis performance and photocleavage properties (chapter 5.2.2). Building blocks which passed these tests were used for synthesis of final caged Synaptobrevin-2 derivative (chapter 5.2.3), and photocleavage as well as binding properties to $\Delta \mathrm{N}$ complexes were investigated (chapter 5.3).

\subsubsection{Synthesis of Photocleavable Amino Acid Building Blocks}

Due to the straightforward syntheses in few steps and UV absorption $>345 \mathrm{~nm}, 6$ - $\underline{\text { Nitroveratryl- }}$ oxycarbonyl (Nvoc) was chosen as PPG for the amino acid building blocks. The simple synthesis and convenient handling outweigh the disadvantages of this caging group as it is used in this work as a proof of principle and to acquire experiences with caged compounds. For the synthesis of soluble caged Synaptobrevin-2 $(28$ - 84) K52K*, R56R*, D57D*, K59K* the following amino acid building blocks are required: Fmoc-Lys(Nvoc)-OH, Fmoc-Arg(Nvoc)-OH and Fmoc-Asp(Nvoc)-OH. Synthesis strategies for Fmoc-Lys(Nvoc)-OH ${ }^{132}$ (16), Fmoc-Asp(Nvoc)$\mathrm{OH}^{[133]}$ and $\operatorname{Arg}(\mathrm{Nvoc})^{[134}$ were already published. Nevertheless, Asp(Nvoc) was shown to be unstable under SPPS conditions ${ }^{[00}$ leading to development of the 3-(4,5-dimethoxy-2nitrophenyl)-2-butyl (DMNPB) moiety as caging group for aspartic (Fmoc-Asp(DMNPB)-OH (27)) and glutamic acid. 107 135] $\operatorname{Arg}$ (Nvoc) was generated by incubation of Orn side chains within the peptide sequence with a so-called guanylating agent to obtain the caged amino acid. ${ }^{134]}$ In this work, the synthesis strategy was transferred to obtain Fmoc-Arg(Nvoc)-OH (21). The synthesised caged amino acid building blocks are summarized in scheme 5.5 and in the following chapters, syntheses of caged amino acid building blocks are described.
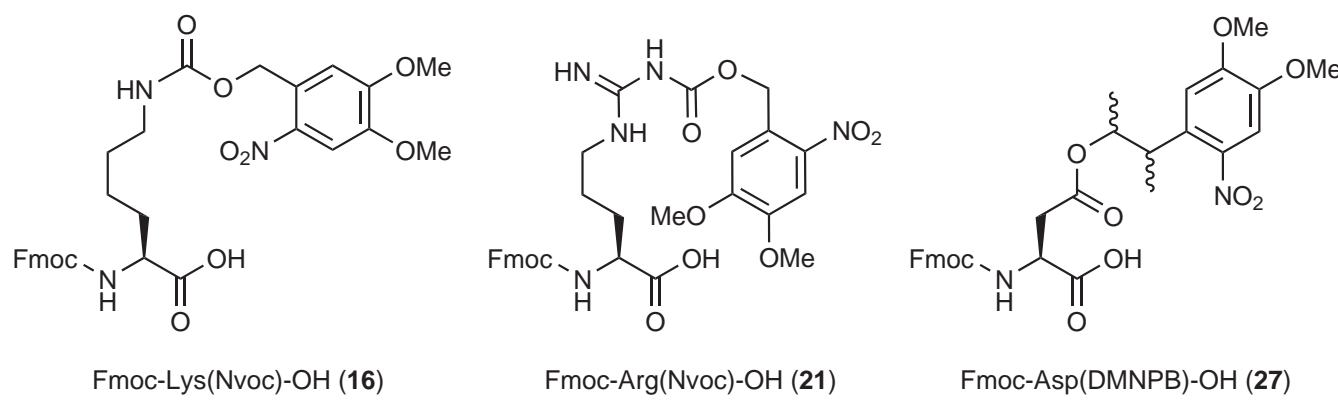

Fmoc-Arg(Nvoc)-OH (21)

Fmoc-Asp(DMNPB)-OH (27)

Scheme 5.5: Caged amino acid building blocks needed for synthesis of soluble caged Syb2: Fmoc-Lys(Nvoc)-OH (16, K(Nvoc) $)^{[132]}$, Fmoc-Arg(Nvoc)-OH (21, R(Nvoc)) and FmocAsp(DMNPB)-OH (27, D(DMNPB)). 107 135]

\section{Synthesis of Fmoc-Lys(Nvoc)-OH (16)}

In the published procedure ${ }^{[132]}$, Fmoc-Lys(Nvoc)-OH (16) was obtained by a two-step synthesis starting from 6-nitroveratrylalcohol $(\mathrm{NV}-\mathrm{OH}, 12)$ via generation of 6-nitroveratryl chloroformate 

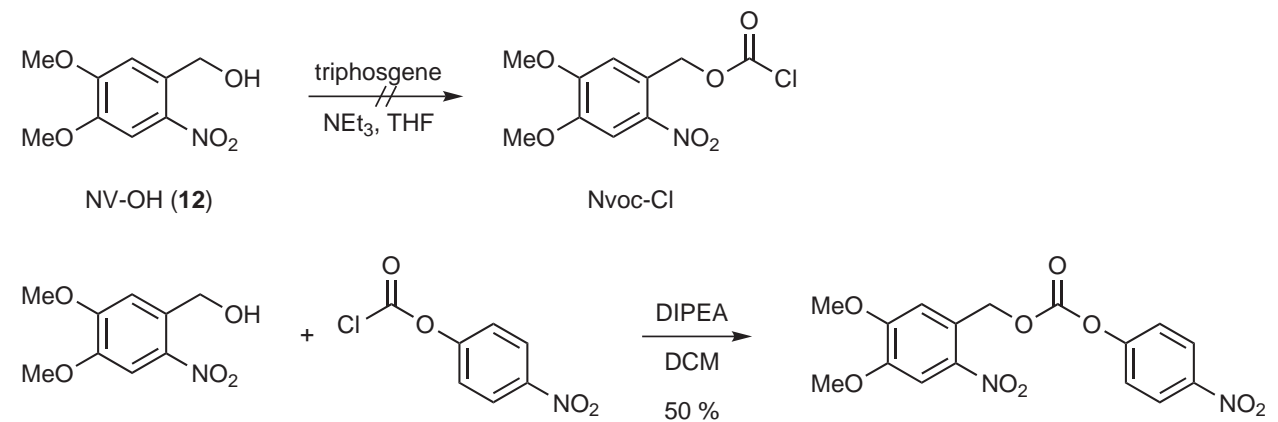

$\mathrm{NV}-\mathrm{OH}(12)$

$p-\mathrm{NPCl}(\mathbf{1 3})$

14<smiles>COc1cc(COC(=O)Oc2ccc([N+](=O)[O-])cc2)c([N+](=O)[O-])cc1OC</smiles>

14

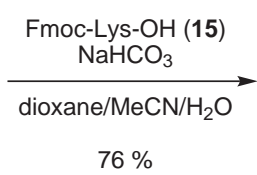

$76 \%$<smiles>COc1cc(COC(=O)NCCCC[C@H](NF)C(=O)O)c([N+](=O)[O-])cc1OC</smiles>

Fmoc-Lys(Nvoc)-OH (16)

Scheme 5.6: Synthesis procedure of the caged Lysine building block Fmoc-Lys(Nvoc)-OH (16). 132 136 [137]

(Nvoc-Cl) and finally substitution by addition of Fmoc-Lys-OH (15) to obtain the target compound (scheme 5.6). For the first step, solution of phosgene in toluene was used in the literature, which was tried to substitute by slightly less toxic triphosgene/triethylamine in THF[136] in this work, but without success. As an alternative, activation of 6-nitroveratrylalcohol (12) was obtained by addition of $p$-nitrophenyl chloroformate $(p-\mathrm{NPCl}, 13)$ and DIPEA in DCM to obtain 6-nitrovertaryl-4'-nitrophenyl carbonate (14). 137] Finally, under slightly basic conditions using $\mathrm{NaHCO}_{3}$, substitution with commercially available Fmoc-Lys-OH (15) in dioxane/MeCN/ $\mathrm{H}_{2} \mathrm{O}(1 / 1 / 1, v / v / v)$ successfully lead to the target compound Fmoc-Lys(Nvoc)$\mathrm{OH}(\mathbf{1 6}, \mathrm{K}(\mathrm{Nvoc}))$ in good yields $(76 \%$, chapter 8.7.1).

\section{Synthesis of Fmoc-Arg(Nvoc)-OH (21)}

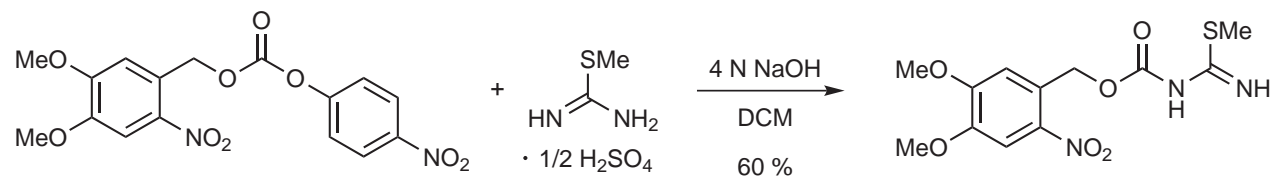

14<smiles>COc1cc(COC(=O)NC(C)=N)c([N+](=O)[O-])cc1OC</smiles>

18<smiles>NCCC[C@H](NC(F)F)C(=O)O</smiles>

Fmoc-Orn-OH (20)

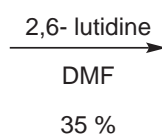<smiles>COc1cc(OC)c([N+](=O)[O-])cc1COC(=O)NC(=N)NCCC[C@H](NC(F)F)C(=O)O</smiles>

Fmoc-Arg(Nvoc)-OH (21)

Scheme 5.7: Synthesis procedure of the caged arginine building block Fmoc- $\operatorname{Arg}(\mathrm{Nvoc})-\mathrm{OH}$ (21). 134137 
The caged arginine amino acid building block was obtained by adapting the published procedure ${ }^{134}$ to the synthesis of a Fmoc building block (scheme 5.7). Originally, the synthesis strategy started from Nvoc-Cl which was also changed to the alternative compound 14 . Substitution with $S$-methylisothiourea semisulfate (17) under basic conditions using $\mathrm{NaOH}$ $(4 \mathrm{~N})$ in DCM yielded the corresponding "guanylating agent" 18 in moderate yields $(60 \%)$. Previous to the next step, Fmoc-Orn(Boc)-OH (19) was deprotected using TFA/DCM (1/1, $v / v)$ to obtain Fmoc-Orn-OH (20) in high yields (99\%). ${ }^{138}$ The side chain of compound 20 was substituted with the "guanylating agent" 18 under basic conditions using 2,6-lutidine in DMF yielding the target compound Fmoc-Arg(Nvoc)-OH (21, R(Nvoc)) (chapter 8.7.2). Due to concerns regarding the TFA stability of this building block, a small amount of $\mathbf{2 1}$ was stirred for $2 \mathrm{~h}$ in TFA and afterwards analysed by TLC and ESI-MS. No instability towards TFA was detected.

\section{Synthesis of Fmoc-Asp(DMNPB)-OH (27)}

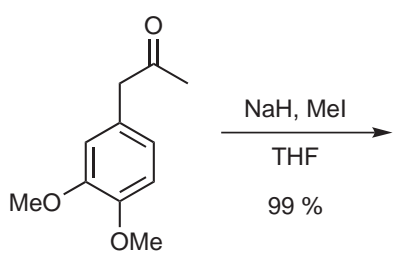

22

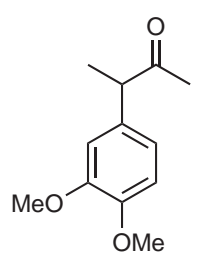

23

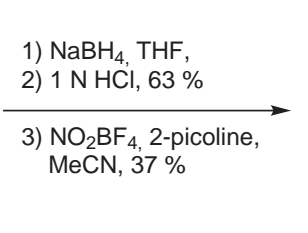

$\mathrm{NO}_{2} \mathrm{BF}_{4}, 2-\mathrm{p}$
$\mathrm{MeCN}, 37 \%$<smiles>C1CCC1</smiles>

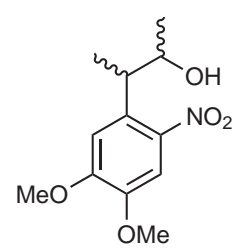

25 (erythro/threo mixture)<smiles>COc1cc([C@@H](C)C(C)O)c([N+](=O)[O-])cc1OC</smiles>

25

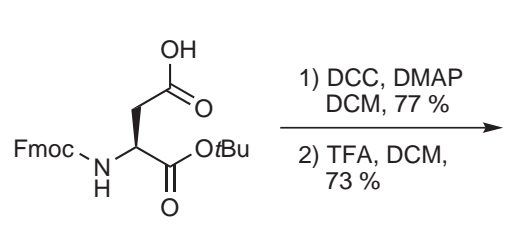

Fmoc-Asp-OtBu (1)

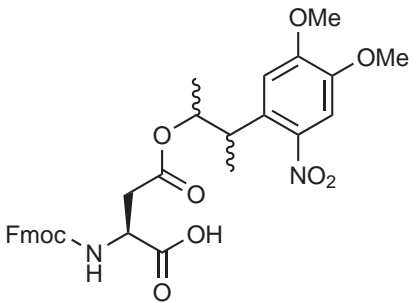

Fmoc-Asp(DMNPB)-OH (26)

Scheme 5.8: Synthesis procedure of the caged aspartic acid building block Fmoc-Asp(DMNPB)$\mathrm{OH}(27)$. 107, 135

The caged aspartic acid building block was obtained by a published procedure 107 135 in five steps (scheme 5.8). Starting from 3,4-dimethoxyphenylacetone (22) which was methylated using sodium hydride and methyl iodide in THF, 3-(3,4-dimethoxyphenyl)-butan-2-one (23) was obtained in excellent yields (99\%). Reduction of ketone 23 to the alcohol 3-(4,5dimethoxphenyl)-butan-2-ol (24) was achieved using sodium borohydride in THF/2-propanol and subsequent addition of $\mathrm{HCl}(1 \mathrm{~N})$, leading to a erythro/threo $(1 / 2)$ mixture of the target compound 24. Nitration in ortho-position was obtained using nitronium tetrafluoroborate and 2-picoline in MeCN, yielding 3-(4,5-dimethoxy-2-nitrophenyl)-butan-2-ol (25). Esterification of alcohol 25 and the unprotected side chain of Fmoc-Asp-OtBu (1) by activation with $N, N^{\prime}$-dicyclohexylcarbodiimide (DCC) and 4-dimethylaminopyridine (DMAP) resulted in the tert-butyl protected caged amino acid Fmoc-Asp(DMNPB)-OtBu (26) in good yields (77\%). 
In the final step, deprotection of $C$-terminal tert-butyl ester was achieved by addition of TFA/DCM $(1 / 1, v / v)$ and the target compound Fmoc-Asp(DMNPB)-OH (27, D(DMNPB)) was obtained in good yields ( $73 \%, 11 \%$ over five steps) (chapter 8.7.3).

\subsubsection{Synthesis and Photocleavage of Caged Test Peptides 28 - 31}

Short Synaptobrevin-2 sequences including caged amino acid building blocks were synthesised by SPPS to test the synthesis performance and photocleavage properties of the caging groups.

\section{Synthesis of Caged Test Peptides 28 - 31}

The following test sequences were synthesised by manual SPPS (chapter 8.4.1): Syb-2 (54 60) R56R(Nvoc) (28), Syb-2 (54 - 60) K59K(Nvoc) (29), Syb-2 (51 - 60) K52K(Nvoc), K59K(Nvoc) (30), and Syb-2 (54-60) D57D(DMNPB) (31). The non-caged sequences Syb-2 (54 - 60) (32), Syb-2 $(51$ - 60) (33) were additionally synthesised for comparison (table 5.9, chapter 8.8.1). Due to the high hydrophobicity of the caging groups, it was avoided to synthesize a test peptide including three caged amino acid building blocks.

Table 5.9: Overview of synthesised caged test peptides $28-31$ and non-caged peptides 32 and 33.

The caged arginine-containing peptide

\begin{tabular}{|c|l|c|}
\hline No. & Syb-2 derivative & Sequence \\
\hline $\mathbf{2 8}$ & Syb-2 (54-60) R56R(Nvoc) & $\begin{array}{c}\text { Nvoc } \\
\text { H-LERDQKL-OH }\end{array}$ \\
\hline $\mathbf{2 9}$ & Syb-2 (54-60) K59K(Nvoc) & $\begin{array}{c}\text { Nvoc } \\
\text { H-LERDQKL-OH }\end{array}$ \\
\hline $\mathbf{3 0}$ & $\begin{array}{l}\text { Syb-2 (51-60) K52K(Nvoc), } \\
\text { K59K(Nvoc) }\end{array}$ & $\begin{array}{c}\text { Nvoc N Noc } \\
\text { H-DKVLERDQKL-OH }\end{array}$ \\
\hline $\mathbf{3 1}$ & Syb-2 (54-60) D57D(DMNPB) & $\begin{array}{c}\text { DMNPB } \\
\text { H-LERDQKL-OH }\end{array}$ \\
\hline $\mathbf{3 2}$ & Syb-2 (54-60) & H-LERDQKL-OH \\
\hline $\mathbf{3 3}$ & Syb-2 (51-60) & H-DKVLERDQKL-OH \\
\hline
\end{tabular}

sequence (28) was synthesised using a batch size of $25 \mu \mathrm{mol}$ on a preloaded Fmoc-Leu-WANG resin LL $(0.32 \mathrm{mmol} / \mathrm{g})$. Single coupling was performed with a reaction time of $30 \mathrm{~min}$ (45 min for Fmoc-Arg(Nvoc)-OH (21)) and successful coupling was checked by performing KAISER test (chapter 8.4.4). After complete synthesis, the peptide was cleaved from the resin, completely deprotected by a TFA cleavage mixture (chapter 8.4.3) and purified by RPHPLC.

For the caged lysine-containing peptide sequences, the synthesis strategy was the following: Firstly, the short sequence Syb-2 (54 - 60) K59K(Nvoc) (29) was synthesised using a batch size of $50 \mu \mathrm{mol}$ on a preloaded Fmoc-Leu-WANG resin LL $(0.32 \mathrm{mmol} / \mathrm{g})$. Single coupling was performed with a reaction time of $30 \mathrm{~min}$ (45 min for Fmoc-Lys(Nvoc)-OH 16) and successful coupling was checked by performing KAISER test. After complete synthesis, the resin was divided into two portions: From portion 1 , the peptide was cleaved, completely deprotected by a TFA cleavage mixture and purified by RP-HPLC. Portion 2 was used for further synthesis of Syb-2 (51 - 60) K52K(Nvoc), K59K(Nvoc) (30) in a batch size of $\sim 25 \mu \mathrm{mol}$ using the same conditions as before.

The caged aspartic acid-containing peptide sequence $\mathbf{3 1}$ was synthesised using a batch size of 
$25 \mu \mathrm{mol}$ on a preloaded Fmoc-Leu-WANG resin LL $(0.32 \mathrm{mmol} / \mathrm{g})$. Just as previously, single coupling was performed with a reaction time of $30 \mathrm{~min}$ (45 min for Fmoc-Asp(DMNPB)-OH 27) and successful coupling was checked by performing KAISER test. After complete synthesis, the peptide was cleaved from the resin, completely deprotected by a TFA cleavage mixture and purified by RP-HPLC.

The non-caged sequences Syb-2 (54 - 60) (32) and Syb-2 (51 - 60) (33) were synthesised using the same strategy as for the two caged lysine-containing peptides $\mathbf{2 9}$ and $\mathbf{3 0}$ but with applying Fmoc-Lys(Boc)-OH instead of Fmoc-Lys(Nvoc)-OH (16) at the corresponding positions.

All peptides were successfully obtained except for Syb-2 (54-60) R56R(Nvoc) (28). After purification by RP-HPLC, a compound was obtained with a difference of mass to charge ratio of $\Delta \frac{m}{z}=-50$ according to ESI-MS which could not be assigned to a possible truncated sequence or other side products. A possible TFA instability of Fmoc-Arg(Nvoc)-OH (21) was excluded previously. Due to this result, two test reactions were performed using Fmoc-Gly-WANG-resin $\mathrm{LL}(0.36 \mathrm{mmol} / \mathrm{g})$ in a batch size of $30 \mu \mathrm{mol}$ each and coupled with Fmoc-Arg(Nvoc)-OH under standard Fmoc-SPPS conditions (scheme 5.10).

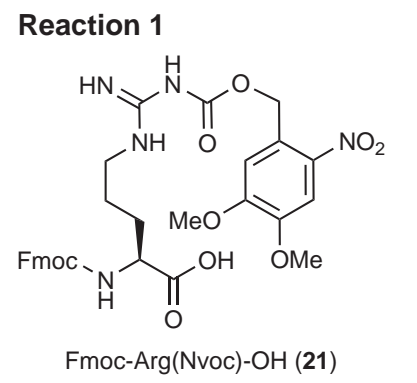

Reaction 2

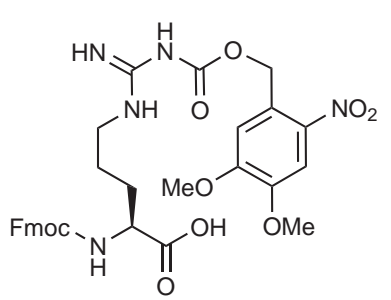

Fmoc-Arg(Nvoc)-OH (21)

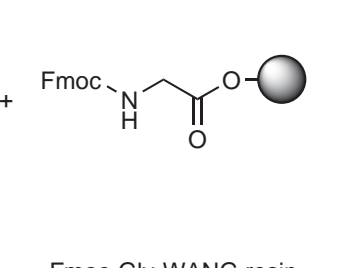

Fmoc-Gly-WANG resin

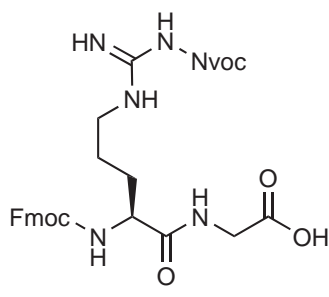

Fmoc-Arg(Nvoc)-Gly-OH
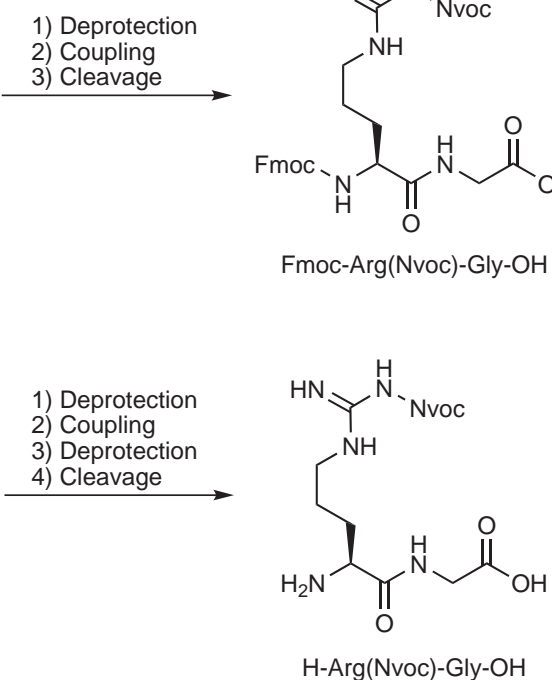

Scheme 5.10: Test reactions investigating stability of Fmoc-Arg(Nvoc)-OH (21) under standard Fmoc-SPPS conditions.

Reaction 1 was performed by deprotection, coupling and cleavage steps, whereas reaction 2 comprised deprotection, coupling, deprotection and cleavage steps. After analytical RP-HPLC no product was detectable by ESI-MS and due to the overall very low yield, especially for reaction 2, NMR spectroscopy was not possible. These results led to the hypothesis, that the building block Fmoc-Arg(Nvoc)-OH (21) might be stable under cleavage conditions, but is probably unstable under coupling and/or deprotection conditions. A second possibility was that the products of the test reactions were soluble in cold diethylether and were therefore 
not precipitated by ether precipitation. Nevertheless, the consequence was that building block $\mathbf{2 1}$ was not suitable for Fmoc-SPPS and could not be used for the synthesis of caged soluble Synaptobrevin-2.

\section{Analysis of Test Peptides 29 - 31 by UV/Vis Spectroscopy}

The successfully synthesised test peptides Syb-2 (54 - 60) K59K(Nvoc) (29), Syb-2 (51 - 60) K52K(Nvoc), K59K(Nvoc) (30), and Syb-2 (54 - 60) D57D(DMNPB) (31) were investigated by UV/vis spectroscopy using the NanoDrop 2000c spectrophotometer from Thermo Fisher Scientific (figure 5.11). The main absorption was observed between $190-260 \mathrm{~nm}$ which is also based on the peptide backbone absorption (figure. 5.11 inset). Two additional absorption maxima were observed for all three test peptides between $275-400 \mathrm{~nm}$ with the following wavelength maxima: Syb-2 (54 - 60) K59K(Nvoc) (29) $300 \mathrm{~nm}$ and $347 \mathrm{~nm}$, Syb-2 (51 - 60) K52K(Nvoc), K59K(Nvoc) (30) 303 nm and 353 nm, Syb-2 (54 - 60) D57D(DMNPB) (31) $304 \mathrm{~nm}$ and $348 \mathrm{~nm}$, respectively. These data supported the suitability of these caging groups for photocleavage at $\lambda>345 \mathrm{~nm}$. Interestingly, a red shift was observed for modification with two Lys(Nvoc) in comparison to one Lys(Nvoc). In addition, the DMNPB caging group exibited a slightly more red shifted absorption maxima than Nvoc.

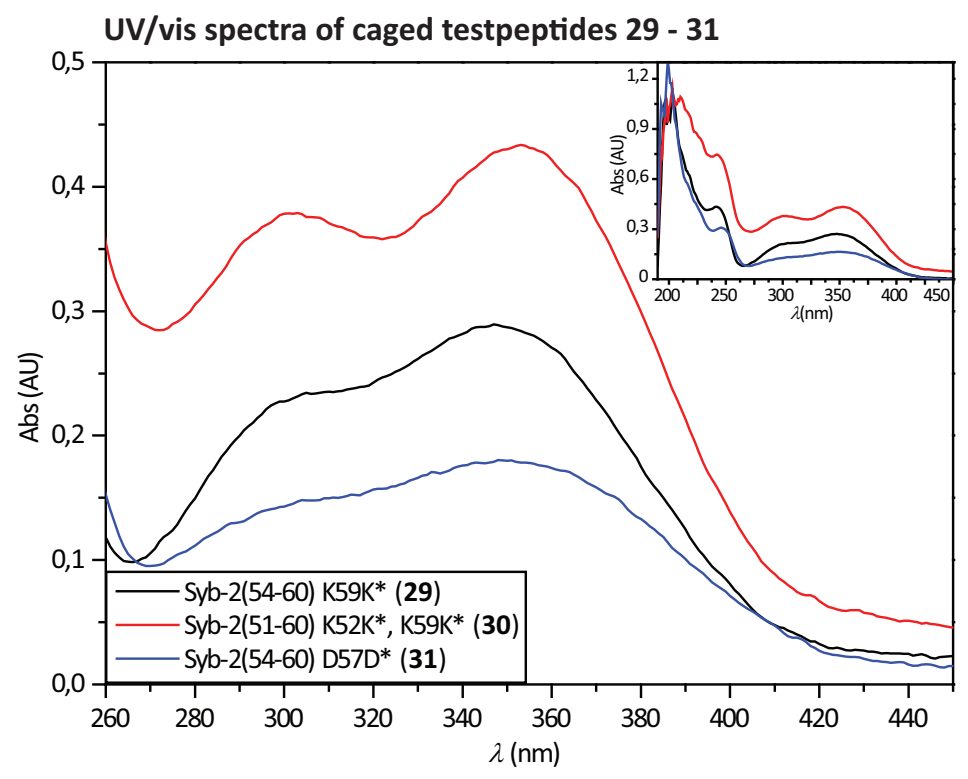

Figure 5.11: UV/vis absorption spectra $(260-450 \mathrm{~nm})$ of caged test peptides $\mathbf{2 9}-\mathbf{3 1}(500 \mu \mathrm{M})$ in $\mathrm{H}_{2} \mathrm{O} / \mathrm{MeCN}(9 / 1, v / v)$ and $\mathrm{H}_{2} \mathrm{O} / \mathrm{MeCN}(1 / 1, v / v)$ for 30, respectively. (inset) $\mathrm{UV} / \mathrm{vis}$ absorption spectra $200-450 \mathrm{~nm}$.

\section{Photocleavage Properties of Test Peptides 29 - 31}

For investigating the photocleavage properties of the caged test peptides $\mathbf{2 9}-\mathbf{3 1}$, the peptides were solved in $\mathrm{H}_{2} \mathrm{O} / \mathrm{MeCN}(9 / 1, v / v$, for 29 and 31; 1/1, v/v, for 30) and diluted to $10 \mu \mathrm{M}$. This concentration represented the highest possible dilution which was still detectable by HPLC. Each sample $(500 \mu \mathrm{L})$ was transferred to a quartz cuevette and irradiated by a mercury UV lamp from Newport equipped with a mirror filter $(280-460 \mathrm{~nm})$ and set to $650 \mathrm{~W}$ for the 
corresponding times (30s $-180 \mathrm{~s})$. Directly after irradiation, the sample was injected and analysed by analytical RP-HPLC. The obtained HPLC chromatograms are summarized in figure 5.12

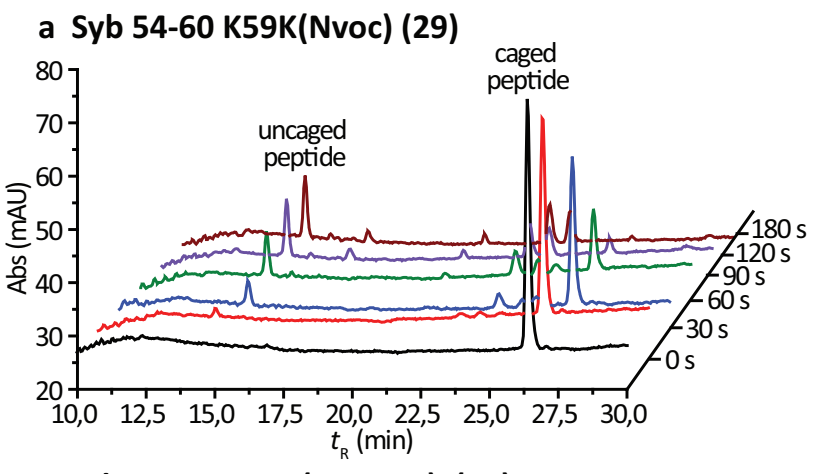

b Syb 51-60 K52K(Nvoc), K59K(Nvoc) (30)

c Syb 54-60 D57D(DMNPB) (31)
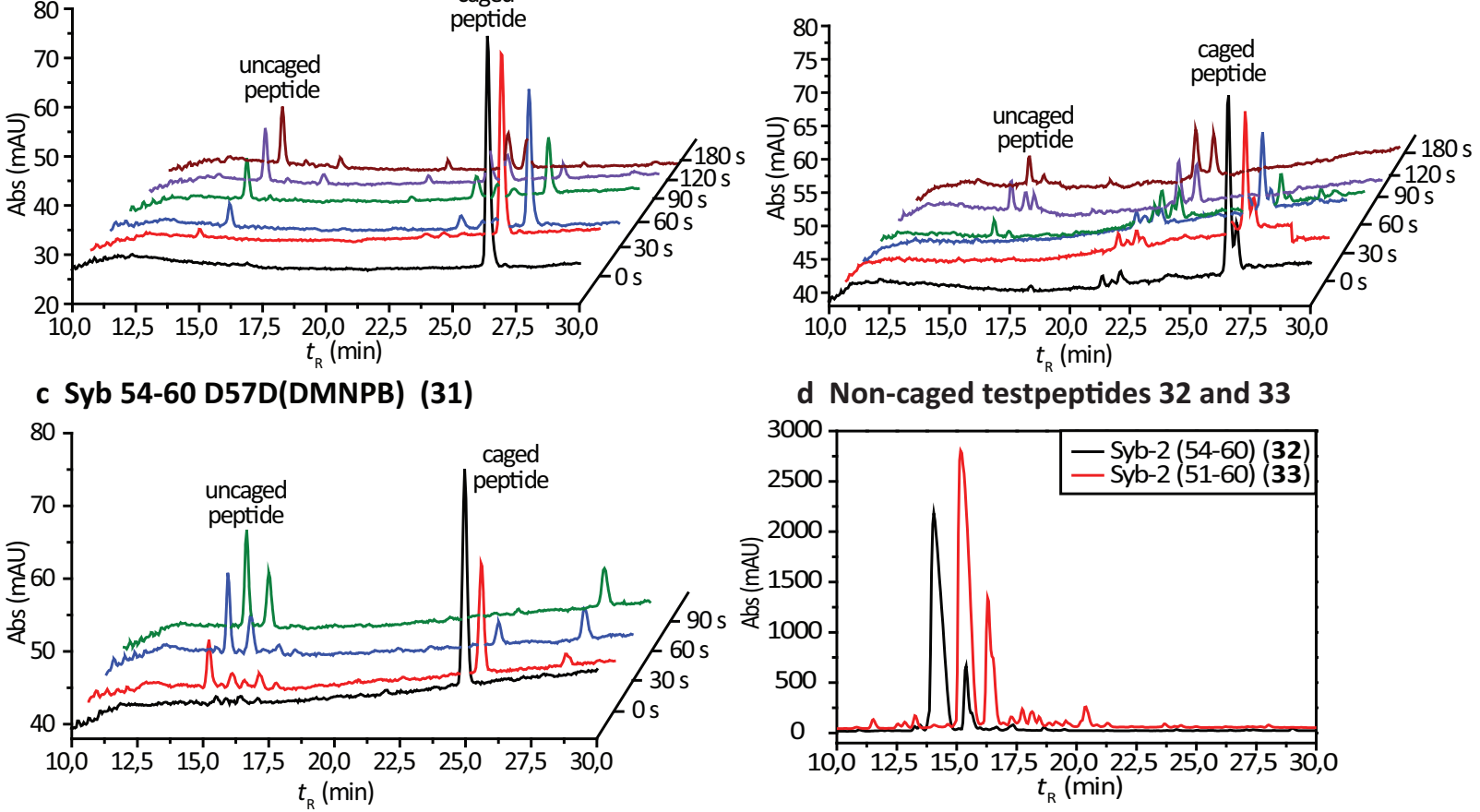

d Non-caged testpeptides 32 and 33

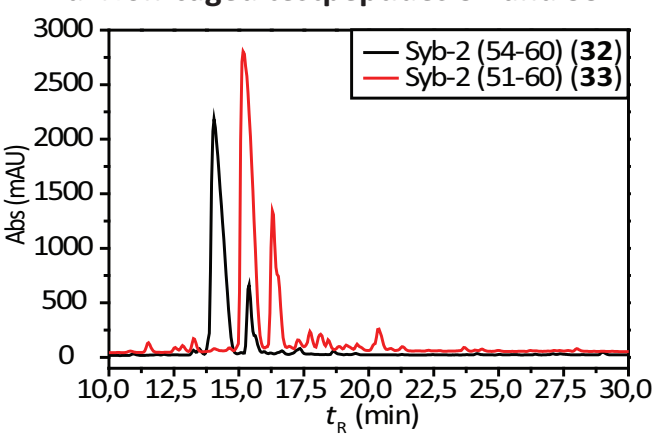

Figure 5.12: Photocleavage experiments of (a) Syb-2 (54 - 60) K59K(Nvoc) (29), (b) Syb-2 $(51-60)$ K52K(Nvoc), K59K(Nvoc) (30), and (c) Syb-2 (54-60) D57D(DMNPB) (31), $10 \mu \mathrm{M}$ each, analysed by analytical RP-HPLC after irradiation for corresponding times (UV traces at $215 \mathrm{~nm}$ ). (d) Analytical RP-HPLC chromatograms of non-caged test peptides Syb-2 (54-60) (32) and Syb-2 $(51-60)$ (33) for comparison of retention times. HPLC gradient: $10-60 \%$ B in $30 \mathrm{~min}$.

All three test peptides were successfully uncaged with this method. The corresponding peak of the caged peptide (cPep) decreased with increasing irradiation times, whereas a peak emerged with a similar retention time as the non-caged peptide. For investigation at which irradiation time a complete photocleavage of the caging groups was obtained, the UV traces were analysed according to the proportion of caged peptide in the sample. Therefore, the corresponding HPLC peaks of the caged peptide were integrated and expressed in percentage to the peak area of the caged peptide at $0 \mathrm{~s}$ (table 5.13).

Almost complete photocleavage was obtained after $180 \mathrm{~s}$ for Syb-2 (54 - 60) K59K(Nvoc) (29), after $120 \mathrm{~s}$ for Syb-2 (51 - 60) K52K(Nvoc), K59K(Nvoc) (30), and most pronounced already after $90 \mathrm{~s}$ for Syb-2 (54 - 60) D57D(DMNPB) (31). Consequently, the DMNPB caging group exhibted faster photocleavage compared to Nvoc, and in addition, with two Nvoc groups considerably more side products were detected (figure $5.12 \mathrm{~b}$ ).

In summary, Fmoc-Lys(Nvoc)-OH (16) and Fmoc-Asp(DMNPB)-OH (27) were proved suitable for synthesis of soluble caged Synaptobrevin-2 for preliminary experiments in vitro. For 
Table 5.13: Proportion of caged peptide for corresponding photocleavage experiments of Syb-2 $(54-60)$ K59K(Nvoc) (29), Syb-2 (51 - 60) K52K(Nvoc), K59K(Nvoc) (30), and Syb-2 (54-60) D57D(DMNPB) (31). Proportions were determined by intergration of corresponding RP-HPLC peaks with the peak of caged peptide at $0 \mathrm{~s}$ set to $100 \%$.

\begin{tabular}{|c|c|c|c|c|c|c|c|}
\hline $\boldsymbol{t}$ in s & 0 & 30 & 60 & 90 & 120 & 180 & Peptides \\
\hline \multirow{2}{*}{$\begin{array}{c}\text { caged } \\
\text { peptide } \\
\text { in \% }\end{array}$} & 100 & 85 & 64 & 26 & 6.5 & 1.5 & $\mathbf{2 9}$ \\
\cline { 2 - 8 } & 100 & 70 & 44 & 16 & 0.7 & 0.5 & $\mathbf{3 0}$ \\
\cline { 2 - 8 } & 100 & 53 & 11 & 2.0 & - & - & $\mathbf{3 1}$ \\
\hline
\end{tabular}

applications in vivo, usage of a different caging groups with faster photocleavage properties in the range of milliseconds to seconds should be considered.

\subsubsection{Synthesis Strategy and Photocleavage of Soluble Caged Synaptobrevin-2 (28 - 84) (36)}

The original design of soluble caged Synaptobrevin-2 (28 - 84) K52K*, R56R*, D57D*, K59K* had to be adapted to the obtained results from syntheses and photocleavage experiments of the caged test peptides. Due to the incompatibility of the building block Fmoc-Arg(Nvoc)-OH (21) to Fmoc-SPPS conditions, it was not possible to modify position R56. Therefore, it was decided to synthesize Syb-2 (28 - 84) K52K(Nvoc), D57D(DMNPB), K59K(Nvoc) (36). The following synthesis strategy was applied: First, the $C$-terminal part Syb-2 $(61-84)(34$, chapter 8.8.2) was synthesised by automated, microwave-assisted SPPS using the CEM LibertyBlue peptide synthesizer according to GSP 2 (chapter 8.4.2). Second, the synthesis was continued under light-exclusion by manual SPPS according to GSP 1 (chapter 8.4.1), including double coupling and elongated coupling times $(2 \times 45 \mathrm{~min})$ for the caged amino acid building blocks $\mathbf{1 6}$ and $\mathbf{2 7}$, to obtain Syb-2 (51 - 84) K52K(Nvoc), D57D(DMNPB), K59K(Nvoc) (35, chapter 8.8.3). The last part of the sequence was again synthesised by automated, microwave-assisted SPPS using the CEM LibertyBlue peptide synthesizer to obtain the target peptide $\mathbf{3 6}$ (chapter 8.8.4). After each synthesis segment, a test cleavage of the resin-bound peptide was performed and synthesis performance analysed by analytical RP-HPLC (figure 5.14).

As expected, the synthesis performance decreased the longer the peptide sequence and lower synthesis performance for the caged amino acid building blocks was observed. Nevertheless, after complete synthesis a huge tendency to peak broadening was detected which might result from possible aggregation of the peptide due to the highly hydrophobic caging groups. In fact, the caging groups had an impact on solubility: Whereas the adamantyl-modified Synaptobrevin-2 derivatives $(\mathbf{6}-\mathbf{1 1})$ were easily soluble in $\mathrm{H}_{2} \mathrm{O} / \mathrm{MeCN}(9 / 1, v / v)$, the caged soluble Synaptobrevin-2 36 was hardly soluble in $\mathrm{H}_{2} \mathrm{O} / \mathrm{MeCN}(1 / 1, v / v)$. Retrospectively, the usage of the new peptide synthesizer CEM LibertyBlue was faster, on the one hand, but on 


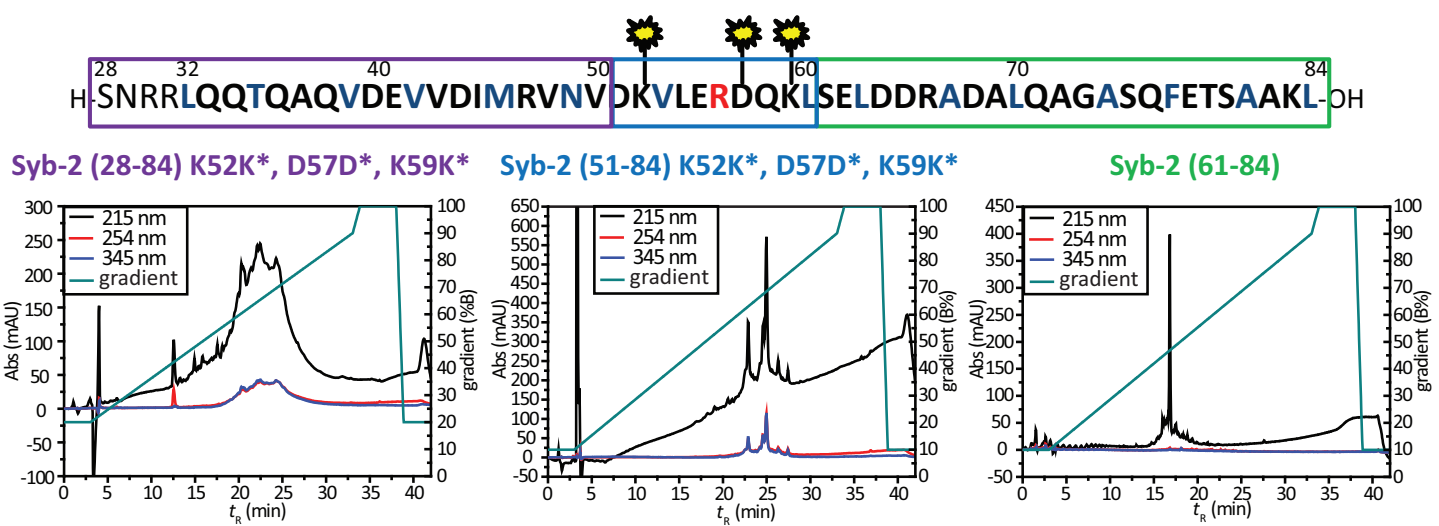

Figure 5.14: Synthesis strategy of soluble caged Synaptobrevin-2 (36) divided into three segments: Syb-2 (61 - 84) (34, green), Syb-2 (51 - 84) K52K(Nvoc), D57D(DMNPB), K59K(Nvoc) (35, blue), and Syb-2 (28 - 84) K52K(Nvoc), D57D(DMNPB), K59K(Nvoc) (36, purple). (bottom) Analytical RP-HPLC chromatograms of the test cleavages after each segment.

the other hand, the missing capping step in the synthesis protocol was a serious disadvantage. Especially for the $N$-terminal part of the synthesis, with possible lesser synthesis performance due to aggregation, this seemed to be crucial for better purification results. Purification of a 56 amino acid long peptide was always challenging, but unfortunately, with additional aggregation, this problem could not be solved by RP-HPLC even with elution at higher temperature $\left(60^{\circ} \mathrm{C}\right)$. Due to time limitations and small amounts, it was tried to purify the peptide only crudely to perform preliminary photocleavage as well as binding experiments. Regarding the high hydrophobicity of the peptide $\mathbf{3 6}$, introduction of less than three and/or more hydrophilic caging groups should be considered for further applications.

\section{Photocleavage Experiments of Soluble Caged Synaptobrevin-2 (36)}

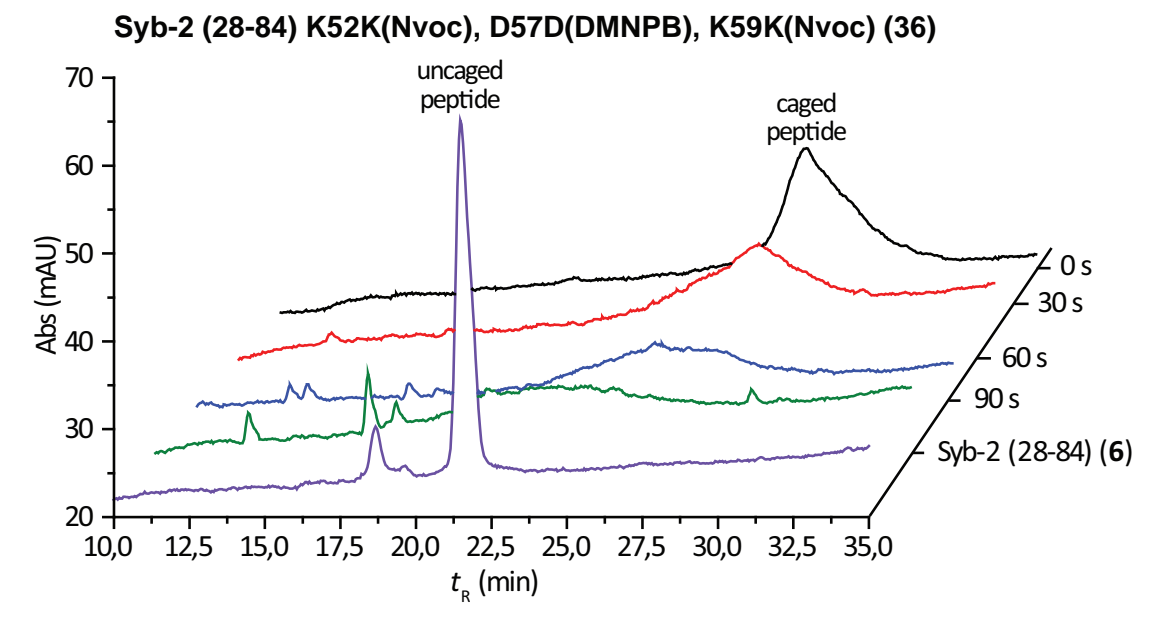

Figure 5.15: Photocleavage experiments of soluble caged Syb-2 $(28-84) \mathrm{K} 52 \mathrm{~K}(\mathrm{Nvoc})$, D57D(DMNPB), K59K(Nvoc) $(36,20 \mu \mathrm{M})$, analysed by analytical RP-HPLC after irradiation for corresponding times (UV traces at $215 \mathrm{~nm}$ ). For comparison, UV trace of Syb-2 $(28-84)$ wild-type (6, purple). HPLC gradient: $20-80 \% \mathrm{~B}$ in $30 \mathrm{~min}$, room temperature. 
Preliminary photocleavage experiments were performed with the crude peptide $\mathbf{3 6}$ using the same protocol as for the caged test peptides 29 - 31. The peptide 36 was solved in $\mathrm{H}_{2} \mathrm{O} / \mathrm{MeCN}$ $(1 / 1, v / v)$ and diluted to $20 \mu \mathrm{M}$ with $\mathrm{H}_{2} \mathrm{O} / \mathrm{MeCN}(9 / 1, v / v)$. The sample $(200 \mu \mathrm{L})$ was transferred to a micro quartz cuvette and irradiated with the mercury UV lamp at $650 \mathrm{~W}$ for the corresponding times. Directly after irradiation, the samples were injected and analysed by analytical RP-HPLC (figure 5.15). The crude peptide $\mathbf{3 6}$ at $0 \mathrm{~s}$ already exhibited a broad peak due to the insufficient purity. With increasing irradiation times, peak broadening increased and shifted to higher polarities, as expected upon cleavage of hydrophobic caging groups. Nevertheless, a distinct peak for the uncaged peptide was not detectable. The proportion of caged peptide for the corresponding times was not possible to investigate due to the huge peak broadening.

\subsection{Binding Studies of Soluble Caged Synaptobrevin-2 $(28-84)$}

Despite the non-satisfactory purity and results of the photocleavage experiments, binding properties of soluble caged Syb-2 (28 - 84) K52K(Nvoc), D57D(DMNPB), K59K(Nvoc) (36) to the $\Delta \mathrm{N}$ complex were investigated by fluorescence anisotropy. As already described in chapter 4.5.2, a soluble, truncated SNARE complex consisting of Syntaxin-1A (183 - 262), SNAP-25 (1 - 206) no Cys mutant, and Synaptobrevin-2 (49 - 96) C79-OG, was used as stabilized acceptor complex. SNARE complex formation properties can be measured indirectly by displacement of the labelled Syb-2 fragment. 1110

Prior to the binding experiments, samples $(100 \mu \mathrm{L})$ of caged Syb-2 36 in $\mathrm{H}_{2} \mathrm{O} / \mathrm{MeCN}(9 / 1, v / v)$ were irradiated with the mercury UV lamp in a micro quartz cuvette for the corresponding times, and afterwards, transferred to EPPENDORF tubes, frozen in liquid nitrogen and transported on ice.

Binding experiments were performed in detergent-containing HEPES buffer, starting with $\Delta \mathrm{N}^{\mathrm{OG}}(80 \mathrm{nM})$ and addition of corresponding caged Syb-2 sample $(125 \mathrm{nM})$ after $\sim 100 \mathrm{~s}$ (chapter 8.3.9). A second addition was performed after $\sim 600 \mathrm{~s}$ (fc. $200-250 \mathrm{nM}$ ). For comparison, binding of Syb-2 (28 - 84) wild-type 6 was performed under the same conditions.

As anticipated, caged soluble Syb-2 (28 - 84) K52K(Nvoc), D57D(DMNPB), K59K(Nvoc) (36) did not bind to the labelled $\Delta \mathrm{N}^{\mathrm{OG}}$ complex as no significant decrease in anisotropy was observed (figure 5.16 red curve). Unfortunately, for the irradiated samples of peptide 36, also no binding was detected as all samples exhibited the same curve shape. Hence, photocleavage was not possible due to the insufficient purity of the peptide. The latter might have also 


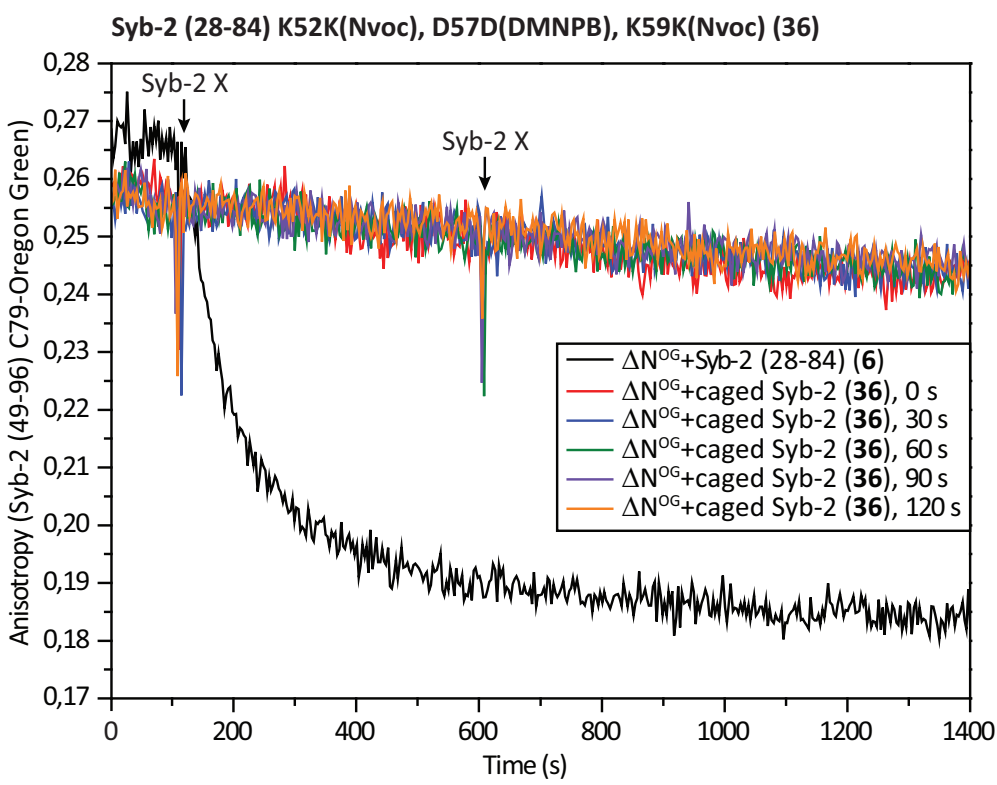

Figure 5.16: Binding experiments of $\Delta \mathrm{N}^{\mathrm{OG}}$ complex and soluble caged Syb-2 (28 - 84) K52K(Nvoc), D57D(DMNPB), K59K(Nvoc) (36) irradiated for corresponding times (0 s (red), $30 \mathrm{~s}$ (blue), $60 \mathrm{~s}$ (green), $90 \mathrm{~s}$ (purple), and $120 \mathrm{~s}$ (orange)) in detergent-containing HEPES buffer. For comparison, binding experiment of $\Delta \mathrm{N}^{\mathrm{OG}}$ and Syb-2 (28 - 84) wild-type (6) (black) performed under the same conditions. First addition of Syb-2 derivatives (125 nM) to the OG-labelled $\Delta \mathrm{N}$ complex $(80 \mathrm{nM})$ was conducted after $\sim 100 \mathrm{~s}$, followed by a second addition of Syb-2 derivatives (fc. $200-250 \mathrm{nM}$ ) after $\sim 600 \mathrm{~s}$ (except for 6). Additions are indicated by arrows. Labelled Syb-2 fragment displacement was traced by the decrease in anisotropy.

contributed to prevention of binding due to truncated and/or defective sequences within the samples. In addition, in a crude peptide sample more unexpected and unwanted side products could be produced by irradiation and photocleavage. Hence, the purification problem of soluble caged Synaptobrevin-2 needs to be solved prior to further applications.

\subsection{Conclusion}

The motivation of these investigations was to design and synthesise a soluble caged Synaptobrevin2 derivative inactive towards SNARE complex zippering, but reactivated upon irradiation. Following the application of caged $\mathrm{Ca}^{2+}$ for in vivo exocytosis investigations, the caged Synaptobrevin-2 should be tested first in vitro but also be applicable for in vivo studies. To design a modified Synaptobrevin-2 analogue with these properties, Synaptobrevin-2 mutant studies were investigated to identify suitable residues for attachment of photocleavable protecting groups. The limitations of suitable amino acid side chains for so-called caging led to the design of caged soluble Syb-2 (28 - 84) K52K*, R56R*, D57D*, K59K*.

As caging group, the 6-nitrovertryloxycarbonyl (Nvoc) moiety was chosen due to the straightforward synthesis protocols of corresponding caged amino acid building blocks comprising 
only few synthesis steps and UV absorption $\lambda>345 \mathrm{~nm}$. The caged amino acid building blocks Fmoc-Arg(Nvoc)-OH (21) and Fmoc-Lys(Nvoc)-OH (16) were synthesised based on previously published synthesis protocols. 132 134 137. For aspartic acid, Fmoc-Asp(DMNPB)-OH (27) was synthesised due to instability of Asp(Nvoc) under SPPS conditions. ${ }^{\text {[90 }}$ 107 135] The synthesised caged amino acid building blocks were successfully incorporated into short test peptide sequences $(29-31)$ by SPPS, except for Fmoc-Arg(Nvoc)-OH (21) which was identified as unstable under SPPS conditions. Nevertheless, UV/vis absorption spectra of test peptides 29 - 31 were measured as well as photocleavage experiments were performed. All test sequences exhibited absorption maxima $\lambda_{\max }>345 \mathrm{~nm}$, and were therefore suitable for possible in vivo experiments. Interestingly, incorporation of two Lys(Nvoc) modifications in one sequence led to a red shift in absorption maxima. Fastest photocleavage was observed for Asp(DMNPB)-containing peptide 31 after $90 \mathrm{~s}$ of irradiation at $650 \mathrm{~W}$. However, for in vivo application complete photocleavage in the time scale of milliseconds to seconds is required. For application of soluble caged Synaptobrevin-2 in vivo, it is necessary to use caging groups with faster photocleavage properties. Preliminary in vitro investigations could be performed with incorporated Nvoc and DMNPB groups.

Based on the results of test peptide synthesis, caged soluble Syb-2 (28 - 84) K52K(Nvoc), D57D(DMNPB), K59K(Nvoc) (36) was synthesised in three segments. Synthesis performance was monitored after each segment by test cleavages and analytical RP-HPLC. Until the second segment, synthesis proceeded with satisfactory performance. Unfortunately, after complete synthesis of the 56 mer peptide sequence 36, purification was even more challenging than for adamantyl-modified Synaptobrevin-2 derivatives $(\mathbf{6}-\mathbf{1 1})$. Possible aggregation of peptide 36 led to highly broadened peaks in RP-HPLC chromatograms. In addition, compared to adamantyl-modified peptides, the caging groups had a great impact on solubility indicating that the hydrophobicity of the peptide significantly increased by the modifications. To decrease this effect, incorporation of less than three or more hydrophilic caging groups needs to be considered. In addition, for synthesis of such long sequences implementation of capping during SPPS is still required for better purification results. Purification by RP-HPLC even at a higher temperature $\left(60^{\circ} \mathrm{C}\right)$ led to only unsatisfactory purity of peptide 36 . Nevertheless, due to time limitations and small amounts, preliminary photocleavage and binding studies were performed. As expected, the crude peptide $\mathbf{3 6}$ exhibited inconclusive results in the photocleavage experiments. With increasing irradiation times even broader peaks were detected and no precise peak for the uncaged peptide could be identified. Consequently, no clear statement about the time needed for complete photocleavage could be made. The binding experiments with labelled $\Delta \mathrm{N}$ complexes exhibited no binding for peptide $\mathbf{3 6}$ which was intended, but for the irradiated samples also no binding was detected. This might be due to the impurity of the sample and/or possible aggregate formation of the caged soluble Syb-2 (28 - 84) K52K(Nvoc), D57D(DMNPB), K59K(Nvoc) (36). The irradiated samples were frozen in liquid nitrogen 
directly after irradiation to stop the radical photocleavage reaction but this procedure might also have supported aggregate formation. Despite overall aggregation tendency, the problem of storage and transportation needs to be solved by a coupled method of UV photocleavage and fluorescence anisotropy measurement in one device. This has the advantage of monitoring photoactivation reactions in faster time scales, and therefore, also fast binding events would be accessible to analyse.

To conclude, for synthesis and in vivo application of caged soluble Synaptobrevin-2, a different caging group should be used with better solubility properties, faster photocleavage and higher absorption wavelength, such as DEACM450. In addition, for the synthesis of these long peptide sequences, the capping step should be maintained during SPPS to enhance purification performance. Lastly, a better suitable UV lamp/laser coupled to a fluorescence spectrometer is necessary to perform and monitor photoactivation reactions simultaneously. 



\section{Synthesis of Labelled Syntaxin Transmembrane Domains for Investigations of Syntaxin Clustering}

A selection of Syntaxin transmembrane domains (TMDs) was synthesised by SPPS, $N$-terminal labelled with different fluorophores (chapter 6.1), and used for fluorescence-based investigations of Syntaxin clustering by Dr. D. Milovanovic, Prof. R. JAhN's work group, Department of Neurobiology, Max-Planck-Institute for Biophysical Chemistry Göttingen (chapter 6.3). 139. 141] In addition, labelled VAMP TMDs were synthesised and provided (chapter 6.2).

\subsection{Synthesis of Labelled Syntaxin TMDs}

The syntheses of Syntaxin (Syx) TMDs were performed by automated and microwave-assisted SPPS using the CEM Liberty12 peptide synthesizer, according to GSP 2 (chapter 8.4.2). Four different sequences were synthesised with different length, mutations and of different isoforms or species (table 6.1): Syntaxin-4 (262 - 297) Homo sapiens (37, chapter 8.9.1), Syntaxin-1A (254 - 288) Homo sapiens (38, chapter 8.9.2), and Syntaxin-1A (254 - 288) K264A, K265A Homo sapiens (39, chapter 8.9.3), Syntaxin-1A (257 - 288) M267A, C271A, I279A Rattus norvegicus ${ }^{142]}$ (40, chapter 8.9 .4 ). Due to the hydrophobic character of the peptides, the corresponding low-loaded WANG resins $(0.30-0.38 \mathrm{mmol} / \mathrm{g})$ were used as well as double coupling and capping were performed.

Table 6.1: Sequences of Syntaxin TMDs $\mathbf{3 7}-\mathbf{4 0}$. The synthesis of peptide $\mathbf{4 0}$ was previously performed by Dr. K. MEYEnBerg. 142]

\begin{tabular}{|c|l|l|}
\hline No. & Name & Sequence \\
\hline 37 & Syx-4 (262- 297) H.S. & H-ALENQKKARKKKVLIAICVSITVVLLAVIIGVTVVG-OH \\
\hline 38 & Syx-1A (254-288) H.s. & H-AVKYQSKARRKKMIIICCVILGIVIASTVGGIFA-OH \\
\hline 39 & $\begin{array}{l}\text { Syx-1A (254 - 288) K264A, } \\
\text { K265A H.s. }\end{array}$ & $\begin{array}{l}\text { H-AVKYQSKARRAAMIIICCVILGIVIASTVGGIFA-OH } \\
\text { C271A, I279A R.n. }\end{array}$ \\
\hline 40 & \begin{tabular}{l} 
H-YQSKARRKKIAIIIACVILGIIAASTIGGIFG-OH \\
\hline
\end{tabular}
\end{tabular}


After complete syntheses, the resin-bound peptides $\mathbf{3 7}$ - $\mathbf{4 0}$ were $N$-terminal labelled with two fluorophores, Atto647N (AttoTec) and Rhodamine Red ${ }^{T M}$ (Invitrogen). These two dyes can be applied as FRET pair due to an overlap of donor emission and acceptor excitation spectra. In addition, peptides $\mathbf{3 8}$ and $\mathbf{4 0}$ were also labelled with Oregon Green $₫ 488$ for photobleaching applications.

\subsubsection{Labelling reaction with Atto647N}

For attachment of the fluorophore Atto647N to the resin-bound and side chain protected peptides $37-40$, the final Fmoc protecting group was removed by piperidine (20\%) in NMP $(2 \times 15 \mathrm{~min})$. After washing of the resin, Atto647N-NHS-ester (AttoTec), benzotriazol-1-yloxytripyrrolidinophosphonium hexafluorophosphate (PyBOP) and DIPEA in NMP were added and the resin was shaken at room temperature under light exclusion overnight (scheme 6.2). The resin was filtrated, washed and the Atto647N-labelled peptides were cleaved from the solid-support and completely deprotected according to GSP 3 (chapter 8.4.3). The precipitated and dried peptides were suspended in $\mathrm{H}_{2} \mathrm{O} / \mathrm{MeCN}(1 / 1, v / v)$, frozen in liquid nitrogen and lyophilised. The detailed synthetic procedures for the Atto647N-labelled Syx TMDs (table 6.5) are described in the following chapters: Atto647N-Syx-4 (262 - 297) Homo sapiens (41, chapter 8.9.5), Atto647N-Syx-1A (254 - 288) Homo sapiens (42, chapter 8.9.6), Atto647NSyx-1A (254 - 288) K264A, K265A Homo sapiens (43, chapter 8.9.7), and Atto647N-Syx-1A (257 - 288) M267A, C271A, I279A Rattus norvegicus (44, chapter 8.9.8).

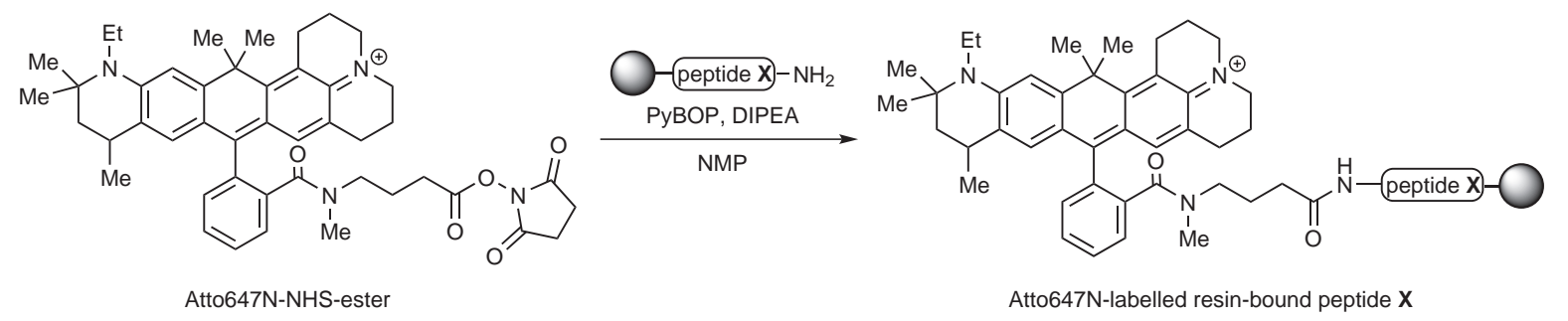

Scheme 6.2: $N$-terminal labelling reaction of a resin-bound and side chain protected peptide $\mathbf{X}$ with Atto647N-NHS-ester (AttoTec). The structure of the fluorophore is patented but was previously published by EGGELING et al. 143]

\subsubsection{Labelling reaction with Rhodamine Red}

For attachment of the fluorophore Rhodamine $\operatorname{Red}^{\mathrm{TM}}$ (RhoRed) to the resin-bound and side chain protected peptides 37, 38 and 40, the final Fmoc protecting group was removed by piperidine $(20 \%)$ in NMP $(2 \times 15 \mathrm{~min})$. After washing of the resin, Rhodamine Red ${ }^{\mathrm{TM}} \mathrm{X}$ succinimidyl ester 5-isomer (Invitrogen) and DIPEA in NMP were added and the resin was shaken at room temperature under light exclusion overnight (scheme 6.3). The resin was filtrated, washed and the RhoRed-labelled peptides were cleaved from the solid-support and 
completely deprotected according to GSP 3 (chapter 8.4.3). The precipitated and dried peptides were suspended in $\mathrm{H}_{2} \mathrm{O} / \mathrm{MeCN}(1 / 1, v / v)$, frozen in liquid nitrogen and lyophilised. The detailed synthetic procedures for the RhoRed-labelled Syx TMDs (table 6.5) are described in the following chapters: RhoRed-Syx-4 (262 - 297) Homo sapiens (45, chapter 8.9.9), RhoRedSyx-1A (254 - 288) Homo sapiens (46, chapter 8.9.10), and RhoRed-Syx-1A (257 - 288) M267A, C271A, I279A Rattus norvegicus (47, chapter 8.9.11).

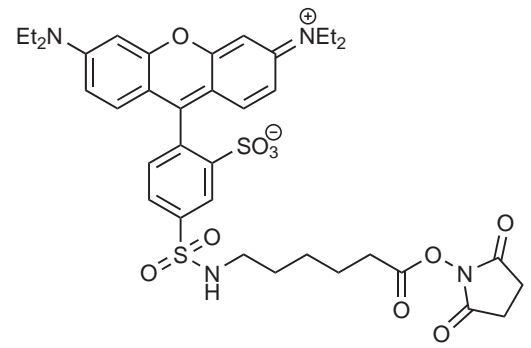

Rhodamine Red-X succinimidyl ester 5-isomer
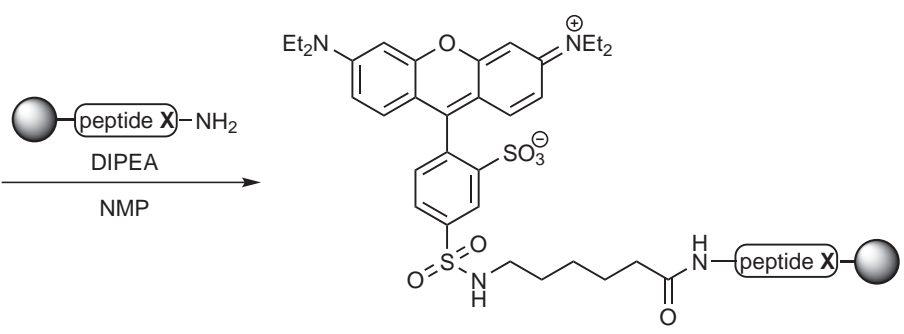

RhoRed-labelled resin-bound peptide $\mathbf{X}$

Scheme 6.3: $N$-terminal reaction of a resin-bound and side chain protected peptide $\mathbf{X}$ with Rhodamine Red- $\mathrm{X}^{\mathrm{TM}}$ succinimidyl ester 5-isomer (Invitrogen).

\subsubsection{Labelling Reaction with Oregon Green}

For attachment of the fluorophore Oregon Green ${ }^{\circledR} 488(O G)$ to the resin-bound and side chain protected peptides 38 and $\mathbf{4 0}$, the final Fmoc protecting group was removed by piperidine (20\%) in NMP ( 2 x 15 min). After washing of the resin, Oregon Green ${ }^{\circledR} 488-X$ succinimidylester 6-isomer (Invitrogen) and DIPEA in NMP were added and the resin was shaken at room temperature under light exclusion overnight (scheme 6.4). The resin was filtrated, washed and the OG-labelled peptides were cleaved from the solid-support and completely deprotected according to GSP 3 (chapter 8.4.3). The precipitated and dried peptides were suspended in $\mathrm{H}_{2} \mathrm{O} / \mathrm{MeCN}(1 / 1, v / v)$, frozen in liquid nitrogen and lyophilised. The detailed synthetic procedures for the OG-labelled Syx TMDs (table 6.5) are described in the following chapters: OG-Syx-1A (254 - 288) Homo sapiens (48, chapter 8.9.12) and OG-Syx-1A (257 - 288) M267A, C271A, I279A Rattus norvegicus (49, chapter 8.9.13).

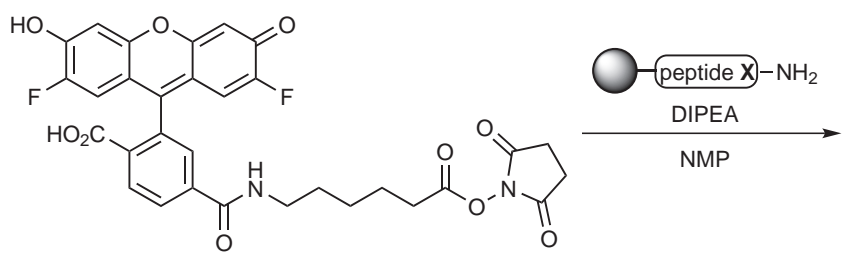

Oregon Green 488-X succinimidyl ester 6-isomer

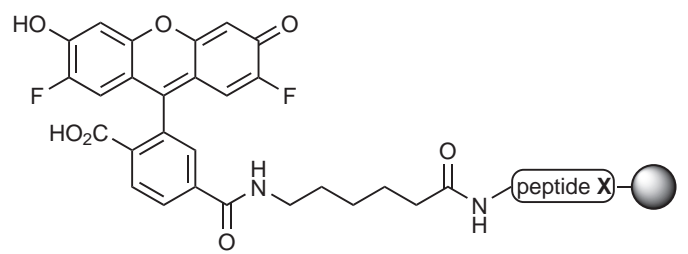

OG-labelled resin-bound peptide $\mathbf{X}$

Scheme 6.4: $N$-terminal reaction of a resin-bound and side chain protected peptide $\mathbf{X}$ with Oregon Green ${ }^{\circledR}$ 488-X succinimidylester 6-isomer (Invitrogen). 
6 Synthesis of Labelled Syntaxin Transmembrane Domains for Investigations of Syntaxin Clustering

\subsubsection{Labelled Syntaxin Transmembrane Domains}

The lyophilised crude peptides were used without further purification. Only the complete peptide sequences were fluorescently labelled due to the protected amino acid side chains and acetylation of possible truncated sequences by the capping step during synthesis. In table 6.5 , all synthesised and labelled Syntaxin TMD sequences are summarised.

Table 6.5: Sequences of labelled Syntaxin TMDs 41 - 49. Synthesis and/or application was

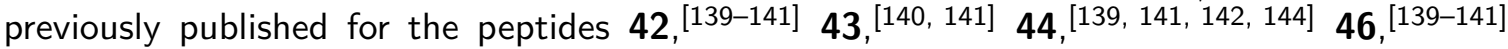
47. 139 141]

\begin{tabular}{|c|c|c|}
\hline No. & Name & Sequence \\
\hline 41 & Atto647N-Syx-4 (262 - 297) H.s. & Atto647N-ALENQKKARKKKVLIAICVSITVVLLAVIIGVTVVG-оH \\
\hline 42 & Atto647N-Syx-1A (254 - 288) H.s. & Atto647N-AVKYQSKARRKKMIIICCVILGIVIASTVGGIFA-OH \\
\hline 43 & $\begin{array}{l}\text { Atto647N-Syx-1A (254 - 288) } \\
\text { K264A, K265A H.s. }\end{array}$ & Atto647N-AVKYQSKARRAAMIIICCVILGIVIASTVGGIFA-OH \\
\hline 44 & $\begin{array}{l}\text { Atto647N-Syx-1A (257 - 288) } \\
\text { M267A, C271A, I279A R.n. }\end{array}$ & Atto647N-YQSKARRKKIAIIIACVILGIIAASTIGGIFG-OH \\
\hline 45 & RhoRed-Syx-4 (262 - 297) H.s. & RhoRed-ALENQKKARKKKVLIAICVSITVVLLAVIIGVTVVG-OH \\
\hline 46 & RhoRed-Syx-1A (254 - 288) H.s. & RhoRed-AVKYQSKARRKKMIIICCVILGIVIASTVGGIFA-OH \\
\hline 47 & $\begin{array}{l}\text { RhoRed-Syx-1A (257 - 288) } \\
\text { M267A, C271A, I279A R.n. }\end{array}$ & RhoRed-YQSKARRKKIAIIIACVILGIIAASTIGGIFG-OH \\
\hline 48 & OG-Syx-1A (254 - 288) H.s. & OG-AVKYQSKARRKKMIIICCVILGIVIASTVGGIFA-OH \\
\hline 49 & $\begin{array}{l}\text { OG-Syx-1A (257 - 288) } \\
\text { M267A, C271A, I279A R.n. }\end{array}$ & OG-YQSKARRKKIAIIIACVILGIIAASTIGGIFG-OH \\
\hline
\end{tabular}

\subsection{Synthesis of Labelled VAMP TMDs}

Analogous to the Syntaxin TMDs, syntheses of VAMP TMDs were performed by automated and microwave-assisted SPPS using the CEM LibertyBlue peptide synthesizer, according to GSP 2 (chapter 8.4.2). Two sequences from different isoforms and one hybrid sequence were synthesised (table 6.6): VAMP-3 (70 - 100)+CVS R.n. (50), VAMP-8 (63 - 100) R.n. (51), and VAMPext (52). Due to the hydrophobic character of the peptides, the corresponding low-loaded WANG resins $(0.31-0.35 \mathrm{mmol} / \mathrm{g})$ were used as well as double coupling procedure. Unfortunately, capping procedure is not possible with the CEM LibertyBlue peptide synthesizer. First, it was intended to synthesise shorter sequences (VAMP-3 (78-100)+CVS R.n. and VAMP-8 $(71-100)$ R.n.) but these peptides were not soluble in trifluoroethanol (TFE) or any other solvent. Hence, elongation of the sequences was necessary. After complete synthesis, the resin-bound peptides $\mathbf{5 0}$ - $\mathbf{5 2}$ were $N$-terminal labelled with the two fluorophores Atto647N and Rhodamine Red ${ }^{\mathrm{TM}}$ analogous to the procedures described in chapters 6.1.1 and 6.1.2. The detailed synthetic procedures for the Atto647N- and RhoRed-labelled VAMP TMDs (table 6.6) 
are described in the following chapters: Atto647N-VAMP-3 (70 100)+CVS Rattus norvegicus (53, chapter 8.9.17), Atto647N-VAMP-8 (63 - 100) Rattus norvegicus (54, chapter 8.9.18), Atto647N-VAMPext (55, chapter 8.9.19), RhoRed-VAMP-3 (70 - 100)+CVS Rattus norvegicus (56, chapter 8.9.20) and RhoRed-VAMP-8 (63 - 100) Rattus norvegicus (57, chapter 8.9.21). With these derivatives investigations of VAMP clustering in membranes by FRET is planned.

Table 6.6: Sequences of synthesised unlabelled and labelled VAMP TMDs $\mathbf{5 0} \mathbf{- 5 7}$.

\begin{tabular}{|c|c|c|}
\hline No. & Name & Sequence \\
\hline 50 & VAMP-3 (70 - 100)+CVS R.n. & $\mathrm{H}-\mathrm{KLKRKYWWKNCKMWAIGISVLVIIVIIIIVWCVS-OH}$ \\
\hline 51 & VAMP-8 (63 - 100) R.n. & H-KVARKFWWKNVKMIVIICVIVLIIVILIILFATGTIPT-он \\
\hline 52 & VAMPext & H-KLKRKYWWKNCKMWAIGISVLVIIVIIIIVWCVSTIPT-OH \\
\hline 53 & $\begin{array}{l}\text { Atto647N-VAMP-3 (70 - 100) } \\
\text { +CVS R.n. }\end{array}$ & Atto647N-KLKRKYWWKNCKMWAIGISVLVIIVIIIIVWCVS-OH \\
\hline 54 & $\begin{array}{l}\text { Atto647N-VAMP-8 } \\
(63-100) \text { R.n. }\end{array}$ & Atto647N-KVARKFWWKNVKMIVIICVIVLIIVILIILFATGTIPT-OH \\
\hline 55 & Atto647N-VAMPext & Atto647N-KLKRKYWWKNCKMWAIGISVLVIIVIIIIVWCVSTIPT-оH \\
\hline 56 & $\begin{array}{l}\text { RhoRed-VAMP-3 (70 - 100) } \\
\text { +CVS R.n. }\end{array}$ & RhoRed-KLKRKYWWKNCKMWAIGISVLVIIVIIIIVWCVS-OH \\
\hline 57 & $\begin{array}{l}\text { RhoRed-VAMP-8 } \\
(63-100) \text { R.n. }\end{array}$ & RhoRed-KVARKFWWKNVKMIVIICVIVLIIVILIILFATGTIPT-OH \\
\hline
\end{tabular}

\subsection{Application of Labelled Syntaxin TMDs}

The synthesised and labelled Syntaxin TMDs were used by Dr. D. Milovanovic, Prof. R. JAHN's work group, Department of Neurobiology, Max-Planck-Institute for Biophysical Chemistry Göttingen for fluorescence-based investigations of Syntaxin clustering. 139. 141]

First, he studied the clustering of Syntaxin in the dependence of membrane thickness. 139 [141] Therefore, he reconstituted the synthesised and labelled Syntaxin-1A TMDs, Atto647N-Syx-1A (254 - 288) H.s. (42) and RhoRed-Syx-1A (254 - 288) H.s. (46), in PC liposomes with different thicknesses and monitored the clustering by a FRET assay. He compared this to Syntaxin-4 TMD clustering by reconstituting Atto647N-Syx-4 (262 - 297) H.s. (41) and RhoRed-Syx-4 (262 - 297) H.s. (45). The Syntaxin-4 isoform exhibited a 1 - 2 amino acid(s) longer TMD than Syntaxin-1A. In combination with imaging ellipsometry, membrane thickness of $3.6 \mathrm{~nm}($ Syx-1A) and $3.7 \mathrm{~nm}$ (Syx-4), respectively, were found to induce the lowest clustering. In eukaryotic cells the plasma membrane exhibits a thickness of $\sim 4 \mathrm{~nm}$, hence, Syntaxin TMD clustering is driven by the mismatch between TMD length and membrane thickness, the so-called hydrophobic mismatch, which was also found to be independent on the presence or absence of cholesterol. 139141

Due to the fact that Syntaxin-1A TMDs exhibit homodimerisation which is known to dependent on specific protein-protein interactions, the labelled dimerisation mutants Atto647N-Syx-1A 
(257 - 288) M267A, C271A, I279A (44) and RhoRed-Syx-1A (257 - 288) M267A, C271A, I279A (47) were used to measure clustering by FRET. The peptides were reconstituted into membranes with hydrophobic mismatch conditions and showed similar clustering properties as for the wild-type. Hence, membrane thickness is more important for clustering than the protein-protein interactions. 139141

Syntaxin-1A as well as Syntaxin-4 include a polybasic motif in the linker region next to the TMD which is known to interact with phosphoinositides, such as phosphatidylinositol 4,5-biphosphate $\left(\mathrm{PI}(4,5) \mathrm{P}_{2}\right)$. In addition, Syntaxin clusters were shown to be enriched with $\mathrm{PI}(4,5) \mathrm{P}_{2}$. To study the co-clustering, Atto647N-Syx-4 TMD (41) and RhoRed-Syx-1A TMD (46) were reconstituted into membranes in the presence or absence of $\mathrm{PI}(4,5) \mathrm{P}_{2}$ and clustering was measured by FRET. The presence of $\mathrm{PI}(4,5) \mathrm{P}_{2}$ increased TMD clustering independently of cholesterol. $139[141]$

Besides $\mathrm{PI}(4,5) \mathrm{P}_{2}, \mathrm{Ca}^{2+}$ ions are also known to enhance Syntaxin clustering. This was confirmed by reconstituting Atto647N-Syx-1A TMD (42) in membranes with matching thickness and lowest clustering. Using stimulated emission depletion (STED) microscopy, it was monitored that clustering was induced by $\mathrm{Ca}^{2+}$ only in the presence of $\mathrm{PI}(4,5) \mathrm{P}_{2}$. Additional FRET experiments with reconstituted Atto647N-Syx-1A TMD (42) and RhoRed-Syx-1A TMD (46) before and after $\mathrm{Ca}^{2+}$ addition confirmed the interaction between the polybasic motif of Syntaxins and $\mathrm{PI}(4,5) \mathrm{P}_{2}$ which is even increased by $\mathrm{Ca}^{2+}$ addition. Mutation of this motif and usage of Atto647N-Syx-1A (254 - 288) K264A, K265A (43) clearly showed a reduced interaction with $\mathrm{PI}(4,5) \mathrm{P}_{2}$ and a reduced effect of $\mathrm{Ca}^{2+}$ addition. 140 141]

In conclusion, it was possible to investigate Syntaxin clustering in membrane model systems dependent on membrane thickness, cholesterol, $\mathrm{PI}(4,5) \mathrm{P}_{2}$ and $\mathrm{Ca}^{2+}$ with the provided synthesised labelled Syx TMDs. 


\section{Summary}

The SNARE-mediated membrane fusion is the essential mechanism for many processes in eukaryotic cells. For example in synaptic transmission, dysfunctions of this basic tool cause neuronal and/or neurodegenerative diseases. Mechanistic insights into this process could offer approaches for development of suitable treatments which might be transferred to other membrane fusion events such as bacterial or viral infections. ${ }^{8}$. Due to the high complexity of the system and despite the extensive research in this field, critical mechanistical questions still remain unanswered. Especially, the mechanisms of SNARE complex zippering and of the SNARE disassembly reaction require further investigations. Recent improvements in cryo-EM provided higher resolution structures of the enzyme NSF and the disassembly machinery, the so-called $20 \mathrm{~S}$ (super)complex. [50 [53] However, crystallisation studies of NSF or the $20 \mathrm{~S}$ complex have not been reported so far, probably due to no effective inhibitor available for the reaction until today.

The first aim of this study was to synthesise an inhibitor for the $\alpha$-SNAP/NSF-mediated disassembly reaction of the SNARE complex. Based on identified interaction sites between $\alpha$-SNAP and Synaptobrevin-2, [64] suitable sequence positions (D51, E55, Q58, E62, D65) of Synaptobrevin-2 were already previously chosen for modification. [119] One or more sterically demanding side chains were introduced on the surface of the SNARE complex to prevent or at least weaken the $\alpha$-SNAP binding which is essential for the disassembly reaction. For the sitespecific modification of the Synaptobrevin-2 SNARE motif by SPPS, the adamantyl-modified amino acid building block Fmoc-Asn(Ad)-OH (4) ${ }^{[119]}$ was synthesised. SNARE assembly and disassembly properties were investigated by time-dependent fluorescence anisotropy. Ternary SNARE complexes with the natural counterparts, Syntaxin-1A and SNAP-25, were successfully formed for all investigated adamantyl-modified Synaptobrevin-2 derivatives. Only for Syb-2 $(28$ - 84) Q58N(Ad) (10), complex formation was more challenging and it proved necessary to use detergent-containing buffer and Syntaxin-1A including its transmembrane domain.

SNARE assembly properties were investigated using a Oregon Green ${ }^{\circledR} 488$ (OG)-labelled stabilised acceptor complex ( $\Delta \mathrm{N}$ complex) which enables to monitor the complex formation indirectly via displacement of the Sby-2 fragment by fluorescence anisotropy. 11] The wild-type sequence Syb-2 (28 - 84) (6) showed comparable SNARE assembly properties to the normally used soluble Syb-2 (1 - 96). The adamantyl-modified Syb-2 (28 - 84) E55N(Ad), D65N(Ad) 
(7) and Syb-2 (28 - 84) Q58N(Ad) (10), however, exhibited clearly retarded complex formation properties. The modifications of these derivatives were close to the $N$-terminal end of the Syb-2 (49-96) fragment used in the $\Delta \mathrm{N}$ complex and might disturb the displacement reaction.

Prior to the disassembly experiments, the ternary SNARE complexes formed from monomeric species were reconstituted into SUVs due to the membrane-dependent efficiency of $\alpha$-SNAP. ${ }^{59}$ The successful complex formation and reconstitution was confirmed by performing a co-floatation assay. The influence of reconstituted modified SNARE complexes on the disassembly reaction mediated by $\alpha$-SNAP and NSF was investigated by time-dependent fluorescence anisotropy. Due to OG-labelled SNAP-25 used for complex formation, $\alpha$-SNAP binding was detected by an increase in fluorescence anisotropy, whereas the complex disassembly upon NSF addition was monitored by a decrease in anisotropy. The adamantyl-modified Syb-2 derivatives should already prevent or at least weaken the $\alpha$-SNAP binding to the SNARE complex. Unfortunately, $\alpha$-SNAP bound to all investigated SNARE complexes containing adamantyl modifications. Especially, Syb-2 (28 - 84) Q58N(Ad) (10) which was previously found to be the most promising derivative for inhibitor development, ${ }^{119]}$ had no effect on $\alpha$-SNAP binding and the disassembly reaction. The modifications of the Synaptobrevin-2 SNARE motif based on a 3/1 stoichiometry of $\alpha$-SNAP/SNARE complex ${ }^{[64}$ were thought to cover two binding sites of $\alpha$-SNAPs. With the recently revealed structure of four $\alpha$-SNAPs binding around the SNARE complex, ${ }^{[50}$ [5] the adamantyl modifications might cover only one $\alpha$-SNAP binding site. In addition, even if one binding site for $\alpha$-SNAP was blocked due to the modifications, the SNARE complex could be disassembled by NSF with less than three or four $\alpha$-SNAPs, like it was shown for the V7-20 S complex. 50 53] Due to the higher time-resolution of fluorescence anisotropy measurements and distinct determination of the reaction by increase/decrease in anisotropy level, the obtained results are summarised as more significant than reaction monitoring by SDS-PAGE. Hence, the introduced adamantyl modifications had no influence on $\alpha$-SNAP binding to the modified SNARE complexes.

The second aim of this study was to study the influence of the adamantyl-modified Syb-2 analogues on the Complexin binding to the SNARE complex, due to the suggested competitive binding of $\alpha$-SNAP and Complexin to the SNARE complex surface. [59] The binding reaction was investigated by time-dependent fluorescence anisotropy and monitored by an increase in anisotropy upon addition of the SNARE complex to OG-labelled Complexin. ${ }^{72}$ Surprisingly, Complexin did bind to the SNARE complex containing Syb-2 (28 - 84) E55N(Ad), D65N(Ad) (7), despite sterically demanding modification of a direct interaction site with Complexin (D65). Probably, the interaction with the neighbouring residues D64 and D68 was able to overcome this disturbance. With respect to studies suggesting a conformational switch region of Synaptobrevin-2 for Complexin to change the orientation of its accessory $\alpha$-helix, 80,82 binding kinetics of Complexin to SNARE complexes containing Syb-2 derivative 7 would be 
interesting to investigate.

More importantly, SNARE complexes containing Syb-2 (28 - 84) Q58N(Ad) (10) were totally inhibiting Complexin binding, although the position of modification was not a direct interaction site and only close to one (D57). The derivative $\mathbf{1 0}$ exhibited the most problems with SNARE complex formation which might lead to the hypothesis that the position Q58 is crucial for SNARE zippering, Complexin binding, and might be energetically critical. Previously suggested as the most promising precursor for inhibition of $\alpha$-SNAP binding, 119] which could not be confirmed by this study, the modification has definitely an impact on SNARE complex properties.

In a third part of this study, the SNARE complex formation itself, the SNARE zippering process was investigated. By introducing photocleavable protecting groups, a soluble caged Synaptobrevin-2 derivative was designed and synthesised which was inactive towards SNARE complex zippering, and could be reactivated upon irradiation. Analogous to the application of caged $\mathrm{Ca}^{2+}$ ions for in vivo exocytosis investigations, the caged Synaptobrevin-2 was preliminary tested in vitro but should also be applicable for in vivo studies. Due to the limitations for attachment of PPGs to suitable amino acid side chains, the soluble caged Syb-2 (28 - 84) K52K(Nvoc), D57D(DMNPB), K59K(Nvoc) was designed. As caging group, the 6-nitroveratryloxycarbonyl (Nvoc) moiety and the related 3-(4,5-dimethoxy-2-nitrophenyl)-2-butyl (DMNPB) moiety were chosen due to straightforward synthetic procedures of corresponding caged amino acid building blocks comprising only few synthesis steps and UV absorption $\lambda>345 \mathrm{~nm}$. The caged amino acid building blocks for SPPS Fmoc-Lys(Nvoc)-OH (16) 132 137 and Fmoc-Asp(DMNPB)-OH (27) 107 135] were synthesised based on previously published synthesis protocols. Short test peptides 29 - $\mathbf{3 1}$ with incorporated caged amino acids were synthesised by SPPS and their photochemical properties investigated by UV/vis spectroscopy as well as photocleavage reactions monitored by RP-HPLC. The fastest uncaging reaction was observed for the Asp(DMNPB)containing peptide 31 after $90 \mathrm{~s}$ of irradiation. For preliminary in vitro investigations of caged Synaptobrevin-2, this was sufficient but for in vivo applications complete photocleavage in the time scale of milliseconds to seconds is required. After synthesis of the complete Syb-2 $(28$ - 84) K52K(Nvoc), D57D(DMNPB), K59K(Nvoc) sequence 36, purification by RP-HPLC was even more challenging than for the adamantyl-modified Syb-2 derivatives $7-11$. Possible aggregation of peptide $\mathbf{3 6}$ led to highly broadened peaks in the RP-HPLC chromatograms which could not be optimised by elution at higher temperature $\left(60^{\circ} \mathrm{C}\right)$. Consequently, peptide 36 was obtained in only unsatisfactory purity to perform preliminary experiments. As expected, the crude peptide $\mathbf{3 6}$ exhibited inconclusive results in the photocleavage experiments and no clear statement about needed irradiation times could be made. Binding experiments with labelled $\Delta \mathrm{N}$ complex monitored by time-dependent fluorescence anisotropy revealed as intended no binding for peptide 36. However, for the irradiated samples of caged Syb-2 (36) also no binding to the $\Delta \mathrm{N}$ complex was detected which is possibly based on the impurity of the 
samples and/or aggregate formation. Due to necessary transportation between irradiation and fluorescence anisotropy measurements, the samples were frozen in liquid nitrogen to stop the radical photocleavage reaction but this procedure might also have supported aggregate formation. Despite overall aggregation tendency, the problem of storage and transportation needs to be solved by a coupled method of UV photocleavage and fluorescence anisotropy measurement in one device. This has the advantage of monitoring photoactivation reactions in faster time scales, and therefore also fast binding events would be accessible to analyse.

To conclude the third part of this study, for in vivo applications of caged soluble Synaptobrevin-2, a caging group with better solubility properties, faster photocleavage and higher absorption wavelength should be used. Despite the considerable expenditure for the synthesis of the amino acid building block, DEACM450[106 112] is a promising candidate for this application. In addition, a better suitable UV lamp/laser coupled to a fluorescence spectrometer is essential to simultaneously perform and monitor photoactivation reactions.

In the last part of this study, Syntaxin transmembrane domains (TMDs) were easily accessible by SPPS and attachment of fluorophores to the $N$-terminal end of the sequence on the solid-support were successfully performed. With the provided synthesised and labelled Syx TMDs, it was possible to investigate Syntaxin clustering in membrane model systems dependent on membrane thickness, cholesterol, $\mathrm{PI}(4,5) \mathrm{P}_{2}$ and $\mathrm{Ca}^{2+}$ by fluorescence-based methods. $139-141$ 


\section{Experimental Part}

\subsection{General Synthetic Methods and Materials}

\section{Reagents}

All starting materials and reagents were of highest grade available and obtained from the following companies: ABCR, Acros-Organics, Alfa Aesar, Bachem, Fisher Scientific, Fluka, Merck, NovaBiochem, Roth, Sigma-Aldrich, TCl and VWR. Amino acids, resins and coupling reagents were purchased from ABCR, Bachem, GL Biochem, IRIS Biotech and NovaBiochem.

\section{Solvents}

All technical solvents were distilled prior to use. Anhydrous solvents of extra dry or puriss. absolute grade (over molecular sieves) were obtained from Acros Organics, Fluka and SigmaAldrich. Acetonitrile and methanol used for HPLC were of the respective grade and purchased from Fisher Scientific, Sigma-Aldrich and VWR. For HPLC and all buffers, water was purified using a Simplicity water purification system from Millipore. All other solvents used were of the grade puriss. p. a. and obtained from Acros Organics, Fisher Chemical, Merck, Roth, Sigma-Aldrich and VWR.

\section{Reactions}

All air- and water-sensitive reactions were conducted under inert atmosphere. For large scale reactions glassware was heat-dried.

\section{Lyophilization}

Freeze-drying of compounds from aqueous solutions containing minimal amounts of methanol or acetonitrile was performed using a Christ-Alpha-2-4 lyophilizer attached to a high vacuum pump. An evacuable Christ RCV-2-18 ultracentrifuge connected to the lyophilizer was used for lyophilization of small volumes from centrifuge tubes. All solutions were frozen with liquid nitrogen before being attached to the lyophilizer.

\section{Chromatography}

Thin Layer Chromatography (TLC)

All reactions were monitored by TLC if possible. TLC was conducted on aluminium backed plates of silica gel $60 \mathrm{~F}_{254}$ (layer thickness: $0.20 \mathrm{~mm}$ ) from Merck. Spots were detected by fluorescence quenching at $254 \mathrm{~nm}$. Non-fluorescence quenching substances were visualized by staining the plate in one of the solutions described in table 8.1 and subsequent careful heating. 
Table 8.1: Staining solutions for Thin Layer Chromatography (TLC)

\begin{tabular}{|c|c|c|c|}
\hline Name & Ninhydrin & MOPS & $\mathrm{PPh}_{3} /$ Ninhydrin \\
\hline Components & $\begin{array}{l}\text { Ninhydrin }(3 \%, w / v) \\
\text { in Ethanol }\end{array}$ & $\begin{array}{l}\left(\mathrm{NH}_{4}\right)_{6} \mathrm{Mo}_{7} \mathrm{O}_{24}(5 \%, w / v) \\
\mathrm{H}_{2} \mathrm{SO}_{4}(10 \%, v / v) \\
\mathrm{CeSO}_{4}(0.3 \%, w / v)\end{array}$ & $\begin{array}{l}\text { 1) } \mathrm{PPh}_{3}(10 \%, w / v) \\
\text { in } \mathrm{DCM} \\
\text { 2) Ninhydrin }(3 \% \text {, } \\
w / v) \text { in Ethanol }\end{array}$ \\
\hline
\end{tabular}

Used for: Amines $\quad$ Most functionalites Azides

\section{Flash Chromatography}

Purification by flash chromatography was conducted using Sigma-Aldrich FLUKA Silica Gel 60 (particle size: $63-200 \mu \mathrm{m}$ ). The crude product was added to the column as adsorbent on threefold amount of silica gel. Eluents are stated in each individual protocol. An overpressure of $0.2-1.0$ bar was applied for the separation.

High performance liquid chromatography (HPLC)

Purification by HPLC was performed at Amersham Pharmacia Biotech systems (Äkta basic, pump-type $P-900$, variable UV detector $U V-900)$. All runs were conducted with a linear gradient of eluent $\mathrm{A}\left(\mathrm{H}_{2} \mathrm{O} /\right.$ TFA $\left.(100 / 0.1, v / v)\right)$ to $\mathrm{B}\left(\mathrm{MeCN} / \mathrm{H}_{2} \mathrm{O} /\right.$ TFA $\left.(80 / 20 / 0.1, v / v / v)\right)$ in $30 \mathrm{~min}$. UV absorption was detected at $215 \mathrm{~nm}, 254 \mathrm{~nm}$ and $280 \mathrm{~nm}$ for regular peptides and at $215 \mathrm{~nm}, 254 \mathrm{~nm}$ and $345 \mathrm{~nm}$ for amino acids or peptides containing caging groups. All columns used are stated in the analysis of the corresponding compound. Flow-rates were $1 \mathrm{~mL} / \mathrm{min}$ for analytical, $3 \mathrm{~mL} / \mathrm{min}$ for semi-preparative and $10 \mathrm{~mL} / \mathrm{min}$ for preparative columns. All samples were dissolved in ultrapure water with a minimum of $\mathrm{MeCN}$ added and filtrated before injection.

\subsection{Characterisation}

\section{Nuclear Magnetic Resonance Spectroscopy (NMR)}

NMR spectra were recorded at Varian instruments (INOVA 600, INOVA 500, VNMRS 300 and Mercury VX 300). Chemical shifts are quoted in ppm (TMS $=0 \mathrm{ppm})$. The resonances of the rest protons of deuterated solvents were taken as internal standards. Multiplicities are abbreviated as follows: $\mathrm{s}=$ singlet, $\mathrm{d}=$ doublet, $\mathrm{t}=$ triplet, $\mathrm{q}=$ quartet, quin = quintet, $\mathrm{m}=$ multiplet, $\mathrm{s}_{\mathrm{br}}=$ broad singlet. Coupling constants $J$ are stated in $\mathrm{Hz} .{ }^{13} \mathrm{C}$ spectra were recorded as broadband decoupled ATP spectra. Data from $[\mathrm{H}, \mathrm{H}]-\mathrm{COSY}, \mathrm{HSQC}$ and HMBC experiments were used to interpret the signals. 


\section{Mass Spectrometry (MS)}

Electron Spray lonisation (ESI) spectra as well as high resolution ESI (HR-MS) spectra were recorded at micrOTOF (ESI-TOF-MS), maXis (ESI-QTOF-MS) or APEX IV (FT-ICR-MS) spectrometer from Bruker Daltonik. The values are given as $m / z$ relations.

\subsection{Analytical and Spectroscopic Methods}

\subsubsection{UV/Vis Spectroscopy}

UV/vis-spectra were recorded with a NanoDrop 2000c spectrophotometer from Thermo Fisher Scientific with a sample size of $2 \mu \mathrm{L}$ per measurement. Blank probe of the corresponding solvent was measured before each set of measurements. Each probe was measured three-times.

\subsubsection{Bradford Assay [145]}

Concentrations of peptides $\mathbf{6}, \mathbf{7}, \mathbf{8}, \mathbf{1 0}$ and $\mathbf{1 1}$ were determined by BRADFORD assay. Standard curve was determined by serial dilution $(0,40,100,400,1000,2000,3000,4000 \mu \mathrm{g} / \mathrm{mL}$ ) of Bovine serum albumin (BSA). All samples $(10 \mu \mathrm{L})$ were mixed with BRADFORD reagent

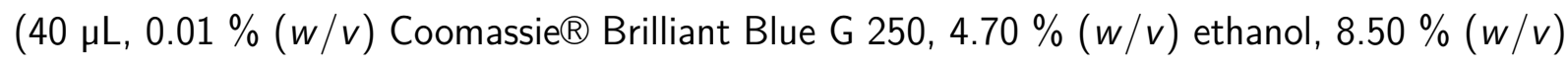
phosphoric acid) and after $5 \mathrm{~min}$ absorption at $595 \mathrm{~nm}$ was measured (five times for standard curve and ten-times for unknown sample) by NanoDrop 2000c spectrophotometer from Thermo Fisher Scientific. Unknown concentrations were calculated and averaged by standard curve.

\subsubsection{BCA Assay[146]}

Concentrations of peptides $\mathbf{6}$ and $\mathbf{3 6}$ were determined by BCA assay. The following reagents were used:

- micro-BCA assay reagent $\mathrm{A}\left(\mathrm{Na}_{2} \mathrm{CO}_{3}(645 \mathrm{~mm})\right.$, NaK Tartrat tetrahydrate $(70 \mathrm{~mm}), \mathrm{pH}$ 11.25),

- micro-BCA assay reagent $B$ (Bicinchoninic acid (BCA, $116 \mathrm{~mm})$ ),

- micro-BCA assay reagent C (copper(II) sulfate pentahydrate $(160 \mathrm{mM})$ ),

- micro-BCA assay solution (reagent $\mathrm{A} / \mathrm{B} / \mathrm{C}(49 / 49 / 2, v / v / v))$.

Standard curve was determined by serial dilution $(0,0.5,2.5,5.0,10,15,20 \mu \mathrm{g})$ of BSA. All samples $(500 \mu \mathrm{L})$ were mixed with micro-BCA assay solution $(500 \mu \mathrm{L})$, vortexed, and incubated $15 \mathrm{~min}$ at $60{ }^{\circ} \mathrm{C}$. Afterwards, samples were cooled to room temperature, vortexed and absorbance at $562 \mathrm{nM}$ was measured five times by NanoDrop 2000c spectrophotometer from Thermo Fisher Scientific. Unknown concentrations were calculated and averaged by standard curve. 


\subsubsection{Photocleavage}

Photocleavage experiments were performed using a mercury UV lamp from Newport equipped with a mirror filter $(280-460 \mathrm{~nm})$ and set to $650 \mathrm{~W}$. For irradiation, the sample $(500 \mu \mathrm{L})$ was transferred to a QS Makro quartz glass cuvette (110-QS, light path: $10 \mathrm{~mm}$, volume: $3500 \mu \mathrm{L}$, outer dimensions: $12.5 \times 12.5 \times 46 \mathrm{~mm}$ ) from Hellma Analytics and positioned centred $\sim 5 \mathrm{~cm}$ in front of the UV lamp opening. For smaller volumes, the sample $(200 \mu \mathrm{L})$ was transferred to a QS Micro Fluorescence Cell quartz glass cuvette (115F-QS, light path: $10 \times 2 \mathrm{~mm}$, volume: $400 \mu \mathrm{L}$, outer dimensions: $12.5 \times 12.5 \times 40 \mathrm{~mm}$ ) and positioned with the longer light path, centred $\sim 5 \mathrm{~cm}$ in front of the UV lamp opening. Irradiation time was varied between $30 \mathrm{~s}$ and $180 \mathrm{~s}$. Directly after irradiation, the sample was analysed by analytical RP-HPLC. For binding studies using $\Delta \mathrm{N}^{\mathrm{OG}}$ complexes (chapter 8.3.9), samples $(100 \mu \mathrm{L})$ of caged Syb-2 (36, $100 \mu \mathrm{M}$ in $\left.\mathrm{H}_{2} \mathrm{O} / \mathrm{MeCN}(1 / 1, v / v)\right)$ were irradiated for corresponding times in the micro quartz cuevette, transferred to EPPENDORF tubes, directly frozen in liquid nitrogen and transported on ice.

\subsubsection{SNARE Protein Constructs, Expression and Purification}

The following purified SNARE proteins were kindly provided by Dr. H. YAVUZ, Prof. R. JAHn's work group, Department of Neurobiology, Max-Planck-Institute for Biophysical Chemistry Göttingen, expressed and purified as previously published: 11 18 6yntaxin-1A (183 - 288), Syntaxin-1A $(180$ - 262), SNAP-25 (1 - 206) no Cys mutant, SNAP-25 (1 - 206) C130OG, Synaptobrevin-2 $(1-96)$ C28-OG, Synaptobrevin-2 $(1-96)$, chinese hamster NSF [59], bovine $\alpha$-SNAP wild-type ${ }^{[59]}$, Complexin I $(1-134)^{[72]}$, Complexin I $(1-134)$ C105S, E39C OG-labeled ${ }^{72]}$. The soluble unlabeled stabilized Q-SNARE acceptor complex ( $\Delta \mathrm{N}$ complex) consisting of Syntaxin-1A (183 - 262), SNAP-25 (no cys) and the C-terminal Synaptobrevin-2 fragment $(46-96)$ as well as the OG-labeled $\Delta \mathrm{N}$ complex $\left(\Delta \mathrm{N}^{\mathrm{OG}}\right)$ consisting of Syntaxin-1A (183 - 262), SNAP-25 (no Cys) and Synaptobrevin-2 (46 - 96) C79-OG were assembled and purified as described earlier. $11,18,60$

\subsubsection{SNARE Complex Assembly}

- Labeled ternary SNARE complex from monomers: For assembly of labeled ternary SNARE complexes, Syntaxin-1A (183 - 288), SNAP-25 (1 - 206) C130-OG and Synaptobrevin-2 $(28-84)$ wt $(\mathbf{6})$ or mutants $(\mathbf{7}, \mathbf{8}, 10$ and $\mathbf{1 1})$ were mixed in molar ratio of 1.0/1.0/1.5 in HEPES buffer $(20 \mathrm{~mm}, \mathrm{NaCl}(300 \mathrm{~mm})$, TCEP $(0.1 \mathrm{~mm})$, CHAPS $(2 \%), \mathrm{pH} 7.4)$ and incubated at room temperature for $2 \mathrm{~h}$ under exclusion of light. The distinct concentrations and components used are described in table 8.2. These complexes were used for disassembly experiments (chapter 4.5.3 and 8.3.9) after co-floatation assays (chapter 4.5.1 and 8.3.8). 
Table 8.2: Components, final concentrations as well as final volume ( $\mathrm{fV}$ ) for SNARE assembly with synthesised Syb-2 (28 - 84) wild-type (6) and adamantyl-modified Synaptobrevin-2 derivatives $(7,8,10,11)$.

\begin{tabular}{|l|c|c|c|c|c|}
\hline Components & $\begin{array}{c}\text { Cplx cont. } \\
(\mathbf{6})\end{array}$ & $\begin{array}{c}\text { Cplx cont. } \\
\text { (7) }\end{array}$ & $\begin{array}{c}\text { Cplx cont. } \\
\text { (8) }\end{array}$ & $\begin{array}{c}\text { Cplx cont. } \\
(\mathbf{1 0 )}\end{array}$ & $\begin{array}{c}\text { Cplx cont. } \\
(\mathbf{1 1})\end{array}$ \\
\hline Syx-1A & $20 \mu \mathrm{M}$ & $25 \mu \mathrm{M}$ & $7.3 \mu \mathrm{M}$ & $25 \mu \mathrm{M}$ & $25 \mu \mathrm{M}$ \\
\hline SN-25-OG & $19 \mu \mathrm{M}$ & $25 \mu \mathrm{M}$ & $7.4 \mu \mathrm{M}$ & $25 \mu \mathrm{M}$ & $25 \mu \mathrm{M}$ \\
\hline Syb-2 X & $27 \mu \mathrm{M}$ & $40 \mu \mathrm{M}$ & $11.2 \mu \mathrm{M}$ & $37 \mu \mathrm{M}$ & $36 \mu \mathrm{M}$ \\
\hline buffer & $40 \mu \mathrm{L}$ & $40 \mu \mathrm{L}$ & $40 \mu \mathrm{L}$ & $40 \mu \mathrm{L}$ & $40 \mu \mathrm{L}$ \\
\hline fV & $141 \mu \mathrm{L}$ & $147 \mu \mathrm{L}$ & $135.5 \mu \mathrm{L}$ & $147 \mu \mathrm{L}$ & $147 \mu \mathrm{L}$ \\
\hline
\end{tabular}

- Unlabeled soluble ternary SNARE complex from $\triangle \mathbf{N}$ complex: For assembly of unlabeled soluble ternary SNARE complexes, $\Delta \mathrm{N}$ complexes $(25 \mu \mathrm{M})$, consisting of Syntaxin-1A $(183$ - 262), SNAP-25 $(1$ - 206) no cys mutant, and the C-terminal Synaptobrevin-2 fragment $(46-96)$, were mixed with Synaptobrevin-2 (28 - 84) wt (6) or mutants (7 or 10) $(37.5 \mu \mathrm{M})$ in HEPES buffer $(20 \mathrm{mM}, \mathrm{NaCl}(300 \mathrm{~mm})$, TCEP $(0.1 \mathrm{~mm}), \mathrm{pH} 7.4)$ and incubated overnight at $4{ }^{\circ} \mathrm{C}$. These complexes were used for Complexin binding studies (chapter 4.6.2, 4.6.3, and 8.3.9). Note that under these conditions no complex formation was obtained for Synaptobrevin-2 (28 - 84) Q58N(Ad) (10).

- Labeled soluble ternary SNARE complex from $\triangle \mathbf{N}$ complex: For assembly of labeled soluble ternary SNARE complexes, $\Delta \mathrm{N}$ complexes $(25 \mu \mathrm{M})$, consisting of Syntaxin$1 \mathrm{~A}(183$ - 262), SNAP-25 $(1$ - 206) no cys mutant, and the C-terminal Synaptobrevin-2 fragment $(46$ - 96) C79-OG, were mixed with Synaptobrevin-2 (28 - 84) wt (6) or mutants (7 or 10) $(37.5 \mu \mathrm{M})$ in HEPES buffer $(20 \mathrm{~mm}, \mathrm{NaCl}(300 \mathrm{~mm})$, TCEP $(0.1 \mathrm{~mm})$, $\mathrm{pH} 7.4)$ and incubated overnight at $4{ }^{\circ} \mathrm{C}$. These complexes were used for rough validation of complex formation by Fluorescence Anisotropy (chapter 4.6.3, and 8.3.9). Note that under these conditions no complex formation was obtained for Synaptobrevin-2 (28 - 84) Q58N(Ad) (10).

- Unlabeled soluble ternary SNARE complex from monomers: For assembly of unlabeled soluble ternary SNARE complexes, Syntaxin-1A $(180-262)(25 \mu \mathrm{M})$, SNAP$25(1-206)$ no cys mutant $(25 \mu \mathrm{M})$, and Synaptobrevin-2 $(28-84)$ wt $(\mathbf{6})$ or mutant (10) $(37.5 \mu \mathrm{M})$ were mixed in HEPES buffer $(20 \mathrm{~mm}, \mathrm{NaCl}(300 \mathrm{~mm})$, TCEP $(0.1 \mathrm{~mm})$, $\mathrm{pH}$ 7.4) and incubated at room temperature overnight. These complexes were used for Complexin binding studies (chapter 4.6.2, 4.6.3, and 8.3.9). Note that under these conditions no complex formation was obtained for Synaptobrevin-2 (28 - 84) Q58N(Ad) (10). 
- Unlabled ternary SNARE complex from monomers: For assembly of unlabeled ternary SNARE complexes, Syntaxin-1A $(183-288)(25 \mu \mathrm{M})$, SNAP-25 $(1-206)$ no cys mutant $(25 \mu \mathrm{M})$, and Synaptobrevin-2 $(28$ - 84) Q58N(Ad) (10) $(37.5 \mu \mathrm{M})$ were mixed in HEPES buffer (20 mM, NaCl (300 mM), TCEP (0.1 mM), CHAPS (1\%), pH 7.4) and incubated at room temperature overnight. These complexes were used for Complexin binding studies (chapter 4.6.3, and 8.3.9).

- Unlabeled binary complex from monomers: For assembly of unlabeled binary complexes SNAP-25 $(1-206)$ no cys mutant $(25 \mu \mathrm{M})$ and Syntaxin-1A $(183-262)(50 \mu \mathrm{M})$ were mixed in HEPES buffer $(20 \mathrm{mM}, \mathrm{NaCl}(300 \mathrm{~mm})$, TCEP $(0.1 \mathrm{~mm}), \mathrm{pH} 7.4)$ and incubated overnight at room temperature. These complexes were used for Complexin binding studies (chapter 4.6.3, and 8.3.9).

- Labeled binary complex from monomers: For assembly of OG-labeled binary complexes SNAP-25 $(1-206)$ C130-OG $(25 \mu \mathrm{M})$ and Syntaxin-1A $(183-262)(50 \mu \mathrm{M})$ were mixed in HEPES buffer $(20 \mathrm{mM}, \mathrm{NaCl}(300 \mathrm{mM})$, TCEP $(0.1 \mathrm{mM}), \mathrm{pH} 7.4)$ and incubated overnight at room temperature. These complexes were used for rough validation of complex formation by Fluorescence Anisotropy (chapter 4.6.3, and 8.3.9).

For SNARE complex assembly with Synaptobrevin-2 (28 - 84) Q58N(Ad) (10), it was crucial to use detergent-containing HEPES buffer $(20 \mathrm{~mm}, \mathrm{NaCl}(300 \mathrm{~mm})$, TCEP $(0.1 \mathrm{~mm})$, CHAPS $(1 \%), \mathrm{pH} 7.4)$ and Syntaxin-1A (183 - 288) to obtain the ternary SNARE complex.

\subsubsection{Reconstitution of SNAREs into Small Liposomes}

SNARE complexes were reconstituted by applying co-micellization method ${ }^{11}[18$. With this method small unilamellar vesicles (SUVs) are obtained by mixing lipids and proteins in a detergent containing buffer and removal of the detergent by size-exclusion chromatography. The used lipid mix consisted of phosphatidylcholine (PC), phosphatidylethanolamine (PE), phosphatidylserine (PS) and cholesterol (molar ratio 5/2/2/1) in HEPES buffer $(20 \mathrm{mM}, \mathrm{KCl}$ (150 mM), EDTA (1 mM), DTT (1 mM), NaCholate $(5 \%, w / v), \mathrm{pH} 7.4)$. Lipid mix and protein mix were combined in a protein to lipid ratio of $1: 1000$ (table 8.3). The concentrations of formed SNARE complexes were roughly estimated by UV absorption measurements at $280 \mathrm{~nm}$ $(1 \mathrm{Abs}=1 \mathrm{mg} / \mathrm{mL})$ using NanoDrop 1000 spectrophotometer from Thermo Fisher Scientific. The mixture with the final Volume ( $\mathrm{V}$ ) was applied to a Sephadex G-50 column equilibrated with detergent free HEPES buffer $(20 \mathrm{~mm}, \mathrm{KCl}(150 \mathrm{mM})$, EDTA (1 mM), DTT (1 mM), pH 7.4). Due to OG-labeled SNARE complexes, collected fraction with highest emission under UV lamp was chosen for further experiments. 
Table 8.3: Concentrations of formed SNARE complexes with synthesised Syb-2 $(28-84)$ wildtype (6) and adamantyl-modified Synaptobrevin-2 derivatives $(\mathbf{7}, \mathbf{8}, \mathbf{1 0}, \mathbf{1 1})$, final concentrations $(\mathrm{fc})$ of lipids and complexes and final volume (fV) of lipid/cplx mixture.

\begin{tabular}{|l|c|c|c|c|c|}
\hline Components & $\begin{array}{c}\text { Cplx cont. } \\
(\mathbf{6})\end{array}$ & $\begin{array}{c}\text { Cplx cont. } \\
\text { (7) }\end{array}$ & $\begin{array}{c}\text { Cplx cont. } \\
\text { (8) }\end{array}$ & $\begin{array}{c}\text { Cplx cont. } \\
\text { (10) }\end{array}$ & $\begin{array}{c}\text { Cplx cont. } \\
\text { (11) }\end{array}$ \\
\hline $\mathrm{c}(\mathrm{cplx})$ & $\sim 18 \mu \mathrm{M}$ & $\sim 14 \mu \mathrm{M}$ & $\sim 8.6 \mu \mathrm{M}$ & $\sim 14 \mu \mathrm{M}$ & $\sim 14 \mu \mathrm{M}$ \\
\hline $\mathrm{fc}($ lipid) & $7.7 \mathrm{mM}$ & $6.9 \mathrm{mM}$ & $5.3 \mathrm{mM}$ & $6.9 \mathrm{mM}$ & $6.9 \mathrm{mM}$ \\
\hline $\mathrm{fc}(\mathrm{cplx})$ & $7.7 \mu \mathrm{M}$ & $6.9 \mu \mathrm{M}$ & $5.2 \mu \mathrm{M}$ & $6.9 \mu \mathrm{M}$ & $6.9 \mu \mathrm{M}$ \\
\hline $\mathrm{fV}($ lipid/cplx) & $157.5 \mu \mathrm{L}$ & $177 \mu \mathrm{L}$ & $179 \mu \mathrm{L}$ & $177 \mu \mathrm{L}$ & $177 \mu \mathrm{L}$ \\
\hline
\end{tabular}

\subsubsection{Co-floatation Assay}

Co-floatation assays were performed as previously published. 18 60] Reconstituted SNARE complexes in SUVs $(50 \mu \mathrm{L})$ were transferred in a centrifuge insert and mixed with Nycodenz ${ }^{\circledR}$ (Axis Shield, $80 \%, 50 \mu \mathrm{L}$ ). A second Nycodenz ${ }^{\circledR}$ layer $(30 \%, 50 \mu \mathrm{L})$ was carefully applied followed by a layer of HEPES buffer $(20 \mathrm{~mm}, \mathrm{KCl}(150 \mathrm{~mm})$, EDTA (1 mM), pH 7.4). Afterwards, the density gradient was centrifuged with a Beckman TL-100 ultracentrifuge (TLS55 rotor, $100000 \times g, 4{ }^{\circ} \mathrm{C}, 1.5 \mathrm{~h}$ ). $20 \mu \mathrm{L}$ aliquots were carefully taken from the top of the gradient (fractions F1 - F8) and analysed by SDS-PAGE. For each analysed SNARE complex, co-floatation assay was performed twice: once for analysis by SDS-PAGE and second for purification before disassembly experiments.

For SDS-PAGE Schaegger gels (Tricin, $10 \%$ ) in Tris buffer (3 M, SDS (0.3 \%), pH 8.45) were used. Electrophoresis was performed with anode buffer (Tris $(0.2 \mathrm{M}), \mathrm{pH} 8.9)$, cathode buffer (Tris $(0.1 \mathrm{M})$, Tricine $(0.1 \mathrm{M})$, SDS $(0.1 \%), \mathrm{pH} 8.25)$ and $120 \mathrm{~V}$ applied. Afterwards, gels were scanned with Typhoon FLA 7000 laser scanner from GE Healthcare with excitation set to $470 \mathrm{~nm}$ and emission to $520 \mathrm{~nm}$ for Oregon Green (OG).

\subsubsection{Fluorescence Anisotropy Experiments}

Anisotropy experiments were carried out as previously published 11$]$ using a Fluorolog 3 (model FL322, Jobin Yvon) spectrometer in T-configuration equipped with in-built polarizers. Excitation and emission wavelengths were set to $488 \mathrm{~nm}$ and $520 \mathrm{mn}$.

- Disassembly experiments: Data was collected with the manufacturer's software. Gfactors were re-measured for every set of epxeriments and slit widths were adjusted to obtain fluorescent counts between 50000 and 100000 . All disassembly experiments were performed at $37{ }^{\circ} \mathrm{C}$ with $5 \mu \mathrm{L}$ of respective sample (combined fractions $\mathrm{F} 1$ and $\mathrm{F} 2$ derived from co-floatation assays) in $600 \mu \mathrm{L}$ of buffer containing HEPES (50 mM), potassium acetate $(20 \mathrm{mM}, \mathrm{KAc})$, potassium glutamate $(120 \mathrm{mM}, \mathrm{KGlu})$ at $\mathrm{pH} 7.4$. Unless stated otherwise, $\mathrm{MgCl}_{2}(5 \mathrm{mM})$ and $2 \mathrm{Na}-\mathrm{ATP}(2 \mathrm{mM})$ were added to activate 
NSF, and $\alpha$-SNAP $(1 \mu \mathrm{M})$ and NSF $(60 \mathrm{nM})$ were added the indicated times. Initial anisotropy of OG-labeled SNARE complexes was measured until equilibration. Upon addition binding or disassembly processes were observed by changes in anisotropy.

- Complexin binding experiments: Data was collected with FluorEssence V3.5 and Gfactor was set to 0.45 for all experiments. Slit widths were adjusted for each experiment to obtain fluorescent counts between 50000 and 100000. All Complexin binding experiments were performed at room temperature in $1.2 \mathrm{~mL}$ of buffer containing HEPES (20 mM), $\mathrm{NaCl}(300 \mathrm{~mm})$, TCEP $(0.1 \mathrm{~mm})$ at $\mathrm{pH} 7.4$. If used SNARE complexes were formed in detergent containing buffer, the same buffer was also used for Complexin binding studies. Initial anisotropy of OG-labeled Complexin was measured until equilibration. Upon addition of SNARE complexes binding processes were observed by changes in anisotropy.

- $\Delta \mathbf{N}$ complex experiments: Data was collected with FluorEssence V3.5 and G-factor was set to 0.45 for all experiments. Slit widths were adjusted for each experiment to obtain fluorescent counts between 50000 and 100000 . All $\Delta N$ complex experiments were performed at room temperature in $1.2 \mathrm{~mL}$ of buffer containing HEPES (20 mM), $\mathrm{NaCl}(300 \mathrm{~mm})$, TCEP $(0.1 \mathrm{~mm})$ and octyl $\beta$-D-glucopyranoside (OctGlu, $1 \%, w / v$ ) at $\mathrm{pH}$ 7.4. Initial anisotropy of OG-labeled $\Delta \mathrm{N}$ complex $(80 \mathrm{nM})$ was measured until equilibration. Upon addition of Synaptobrevin-2 (1 - 96), Synaptobrevin-2 (28 - 84) wt or mutants $(\mathbf{6}, \mathbf{7}, \mathbf{1 0}$ and 36) dissociations of OG-labeled Synaptobrevin-2 (49-96) fragment were detected by changes in anisotropy. The used concentrations and addition times are stated in Fig. 4.10 and 5.16 .

\subsection{General Synthesis Procedures (GSP)}

\section{Solid Phase Peptide Synthesis}

For all peptide syntheses preloaded WANG resin with low loading density $(0.3-0.4 \mathrm{mmol} / \mathrm{g})$ was used for synthesis. The following amino acid building blocks were used in the presented peptide syntheses:

Fmoc-Ala-OH, Fmoc-Arg(Pbf)-OH, Fmoc-Asn(Trt)-OH, Fmoc-Asp(OtBu)-OH, Fmoc-Cys(Trt)$\mathrm{OH}$, Fmoc-Gln(Trt)-OH, Fmoc-Glu(OtBu)-OH, Fmoc-Gly-OH, Fmoc-lle-OH, Fmoc-Leu-OH, Fmoc-Lys(Boc)-OH, Fmoc-Met-OH, Fmoc-Phe-OH, Fmoc-Ser(tBu)-OH, Fmoc-Thr( $t \mathrm{Bu})-\mathrm{OH}$, Fmoc- $\operatorname{Tyr}(t \mathrm{Bu})-\mathrm{OH}$, Fmoc-Val-OH. Unnatural amino acids are used as stated in the protocols. 


\subsubsection{GSP 1: Manual Solid Phase Peptide Synthesis[124]}

For manual solid phase peptide synthesis an acid- and base-resistant Becton Dickinson Discardit $^{T M}$ // (BD)-syringe equipped with a polyethylene-frit was used as reaction vessel. The resin was swollen in NMP ( $\sim 10 \mathrm{~mL} / \mathrm{g}$ resin) by shaking for at least $1 \mathrm{~h}$ before starting reaction. The batch size was varied for each experiment, the protocol shown here is for $0.05 \mathrm{mmol}$ (about $250 \mathrm{mg}$ of resin) in a $2 \mathrm{~mL}-B D$-syringe.

1. Deprotection: The Fmoc protecting group was removed with piperidine $(20 \%, v / v)$ in NMP $(2 \times 2 \mathrm{~mL})$ for $15 \mathrm{~min}$ each. Afterwards, the resin was washed with NMP $(5 \times 2 \mathrm{~mL}), \mathrm{DCM}(5 \times 2 \mathrm{~mL})$ and NMP $(5 \times 2 \mathrm{~mL})$.

2. Coupling: The respective amino acid (5.00 eq.) was dissolved in NMP (0.2 M) and HBTU/HOBt (4.90/5.00 eq., 0.49/0.50 M in DMF) was added. Directly before transferring the coupling mixture to the resin DIPEA (10.0 eq.) was added to activate the mixture. The resin was shaken for $30-60$ min (dependent on used amino acid and sequence-length). For double coupling, the amino acid mixture was added to the resin a second time before washing. Afterwards, the resin was washed with NMP $(5 \times 2 \mathrm{~mL})$, $\operatorname{DCM}(5 \times 2 \mathrm{~mL})$ and NMP $(5 \times 2 \mathrm{~mL})$.

3. Capping: $A$ solution of $\mathrm{Ac}_{2} \mathrm{O}(10 \%, v / v)$ and DIPEA $(5 \%, v / v)$ in NMP $(2 \times 2 \mathrm{~mL})$ was added to the resin and shaken for $10 \mathrm{~min}$ each. Afterwards, the resin was washed with NMP $(5 \times 2 \mathrm{~mL}), \mathrm{DCM}(5 \times 2 \mathrm{~mL})$ and NMP $(5 \times 2 \mathrm{~mL})$.

4. Repetition: Steps $1-3$ were repeated as often as required to complete the sequence. In case of longer breaks during synthesis the resin was washed with DCM $(10 \times 2 \mathrm{~mL})$ and dried under reduced pressure. After complete synthesis, the last Fmoc protecting group was removed according to step 1 and the resin was washed with NMP $(10 \times 2 \mathrm{~mL})$, DCM $(10 \times 2 \mathrm{~mL})$ and dried under reduced pressure.

\subsubsection{GSP 2: Automated Solid Phase Peptide Synthesis}

\section{CEM Liberty12 peptide synthesizer}

Automated solid phase peptide synthesis was conducted by using standard-Fmoc-protocol at a microwave-supported peptide synthesizer Liberty12 from CEM (Kamp-Lintfort, Germany) which consist of a Liberty unit and a Discover microwave. All resins, reagents and solvents necessary for the reaction were connected to the Liberty unit. In the case of the Liberty 12 unit it was possible to attach 20 natural and five unnatural amino acids as well as twelve resins. All amino acids were applied as $0.2 \mathrm{M}$ solutions in NMP. Standard reagents were used for deprotection (piperidine $(20 \%, v / v)$ in NMP), coupling (HBTU/HOBt $(0.5 \mathrm{M})$ in DMF, DIPEA $(2.0 \mathrm{M})$ in NMP), capping $\left(\mathrm{Ac}_{2} \mathrm{O}(20 \%, v / v)\right.$ in NMP) and washing (NMP). All reactions were conducted microwave-supported and under nitrogen. The standard cycle for the batch size of 
$0.10 \mathrm{mmol}$ with double coupling procedure (0.10-Double-Capping) was used if not stated in the corresponding synthesis protocol. The standard cycle consisted of two deprotection steps (30 s, $40{ }^{\circ} \mathrm{C}, 35 \mathrm{~W}$ and $\left.180 \mathrm{~s}, 75^{\circ} \mathrm{C}, 35 \mathrm{~W}\right)$, two coupling steps $\left(120 \mathrm{~s}, 75^{\circ} \mathrm{C}, 25 \mathrm{~W}\right.$ and $300 \mathrm{~s}, 75^{\circ} \mathrm{C}$, $25 \mathrm{~W})$, a capping reaction $\left(180 \mathrm{~s}, 75^{\circ} \mathrm{C}, 25 \mathrm{~W}\right)$ and washing (NMP) in between and afterwards. For the amino acids Cys (0.10-Double-Capping 50C) and Arg (0.10-Double-Capping Arg (KM)) special programs were used as well as for long sequences for the $\mathrm{N}$-terminal amino acids $(0.10$ Double-Capping $10 \mathrm{~min}$ ). At the end of the synthesis, the terminal Fmoc protecting group was removed. After completion of the automated synthesis, the resin was transferred from a Falcon Tube into a $10 \mathrm{~mL}-B D$-syringe, washed with DCM $(15 \times 8 \mathrm{~mL})$ and dried under reduced pressure.

\section{CEM LibertyBlue peptide synthesizer}

Automated solid phase peptide synthesis was conducted by using standard-Fmoc-protocol at a microwave-supported peptide synthesizer LibertyBlue from CEM (Kamp-Lintfort, Germany) which consist of a Liberty unit and a Discover microwave. All reagents and solvents necessary for the reaction were connected to the Liberty unit. In the case of the LibertyBlue unit it was possible to attach 20 natural and seven unnatural amino acids. All amino acids were applied as $0.2 \mathrm{M}$ solutions in DMF. Standard reagents were used for deprotection (piperidine $(20 \%$, $v / v), \mathrm{HOBt}(0.1 \mathrm{M})$ in DMF), coupling (DIC (0.5 M) in DMF, Oxyma $(1.0 \mathrm{M})$ in DMF) and washing (NMP). All reactions were conducted microwave-supported and under nitrogen. The standard cycle for the batch size of $0.10 \mathrm{mmol}$ with double coupling procedure for high-swelling resins (0.10-Double Coupling (HS)) was used if not stated in the corresponding synthesis protocol. The standard cycle consisted of one deprotection step $\left(15 \mathrm{~s}, 75{ }^{\circ} \mathrm{C}, 155 \mathrm{~W}\right.$, followed by $\left.50 \mathrm{~s}, 90{ }^{\circ} \mathrm{C}, 35 \mathrm{~W}\right)$, two coupling steps $\left(15 \mathrm{~s}, 75^{\circ} \mathrm{C}, 170 \mathrm{~W}\right.$ and $\left.110 \mathrm{~s}, 90^{\circ} \mathrm{C}, 30 \mathrm{~W}\right)$ and washing (NMP) between deprotection and coupling. For the amino acids Cys (0.10-Double $50^{\circ} \mathrm{C}$ Coupling (HS)) and Arg (0.10-Double Arg Coupling (HS)) special programs were used. Deprotection and coupling steps for Cys were proceeded at max. $50{ }^{\circ} \mathrm{C}$. Arg was coupled with elongated coupling time and decreased temperature $\left(25 \mathrm{~min}, 25^{\circ} \mathrm{C}, 0 \mathrm{~W}\right.$ and $120 \mathrm{~s}, 75^{\circ} \mathrm{C}$, $30 \mathrm{~W}$ ). After completion of the automated synthesis the resin was transferred from the reaction vessel in a $10 \mathrm{~mL}-B D$-syringe, washed with $\mathrm{DCM}(15 \times 8 \mathrm{~mL})$ and dried under reduced pressure.

\subsubsection{GSP 3: Peptide Cleavage from Solid-Support ${ }^{[124]}$}

For peptide cleavage from solid-support and deprotection of amino acid side chains a solution of TFA, TIS and $\mathrm{H}_{2} \mathrm{O}(95 / 2.5 / 2.5, \mathrm{v} / \mathrm{v} / \mathrm{v})$ was added $10 \mathrm{~mL} / \mathrm{g}$ to the dry resin and shaken for $2 \mathrm{~h}$ at room temperature. In the case of Cys or Met within the peptide sequence a solution of TFA, TIS, EDT and $\mathrm{H}_{2} \mathrm{O}(94 / 1.0 / 2.5 / 2.5, v / v / v / v)$ was used. The solution was collected and the resin was washed with TFA $(3 \times 10 \mathrm{~mL} / \mathrm{g})$. The solvent was removed by nitrogen stream and the peptide precipitated in cold $\mathrm{Et}_{2} \mathrm{O}(2-10 \mathrm{~mL})$, suspended by ultrasonic bath, stored for $20-30 \mathrm{~min}$ at $-18{ }^{\circ} \mathrm{C}$ and centrifugated (14000 rpm, $\left.-18{ }^{\circ} \mathrm{C}, 10 \mathrm{~min}\right)$. The ether 
was decanted and the precipitation repeated twice. The crude peptide was dried under reduced pressure and purified by HPLC.

\subsubsection{GSP 4: Kaiser Test ${ }^{[147]}$}

Successful amino acid coupling during manual SPPS (chapter 8.4.1) was checked by performing KAISER test ${ }^{147]}$. After coupling step and washing a small amount of the resin was washed again with DCM, dried and a few drops of the following solutions were added in that order:

1. Phenol $(42.5 \mathrm{M})$ in $\mathrm{EtOH}$,

2. Ninhydrin $(0.3 \mathrm{M})$ in $\mathrm{EtOH}$,

3. $\mathrm{KCN}(20.0 \mu \mathrm{M})$ in pyridine.

The mixture was heated for $5 \mathrm{~min}$ to $120^{\circ} \mathrm{C}$. In the case of blue coloured solution, the coupling step was repeated due to evidence of primary amines. A successful coupling was achieved, if the solution showed a yellow colour. 147 . 


\subsection{Synthesis of Adamantyl Modified Amino Acid Building Block (4) ${ }^{[119]}$}

(S)-(tertbutoxycarbonyl)-2-(9-fluorenylmethyloxycarbonyl)-4-(1-adamantylamine)-oxobutane (3)<smiles>O=C(O)C[C@H](NC(F)F)C(=O)OCCOCCNC1(Cl)C2CC3CC(C2)CC1C3</smiles>

Fmoc-Asp-OtBu (1)

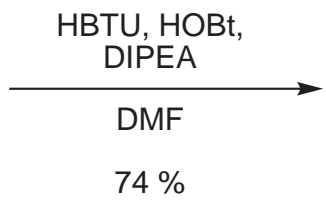

2

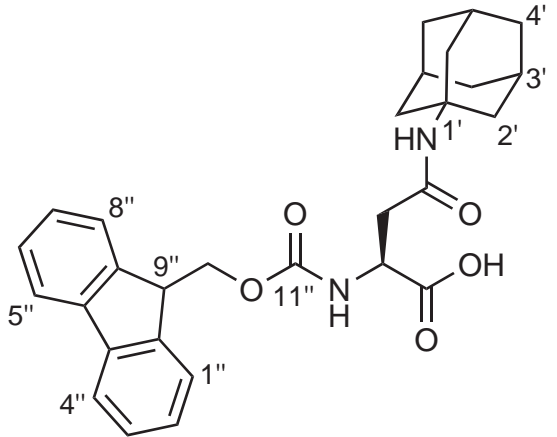

Fmoc-Asn(Ad)-OtBu (3)

$\mathrm{C}_{33} \mathrm{H}_{40} \mathrm{~N}_{2} \mathrm{O}_{5}$

$544.7 \mathrm{~g} / \mathrm{mol}$

N-Fmoc-Asp-OtBu (1, 1.00 g, 2.43 mmol, 1.50 eq. $)$, HOBt (0.33 g, 2.43 mmol, 1.50 eq.) and HBTU (0.92 g, $2.43 \mathrm{mmol}, 1.50$ eq.) were dissolved in dry DMF (5 mL) under argon atmosphere and activated by addition of DIPEA $(0.55 \mathrm{~mL}, 0.42 \mathrm{~g}, 3.24 \mathrm{mmol}, 2.00$ eq.). The reaction mixture was stirred for $5 \mathrm{~min}$ before addition of 1 -adamantylamino hydrochloride $(2$, $0.30 \mathrm{~g}, 1.62 \mathrm{mmol}, 1.00 \mathrm{eq}$.) in dry DMF (3 mL). The reaction was stirred overnight at room temperature. The solvent was removed under reduced pressure and the residue dissolved in EtOAc $(30 \mathrm{~mL})$. The organic layer was washed with $\mathrm{NH}_{4} \mathrm{Cl}$ solution $(0.1 \mathrm{M}, 3 \times 25 \mathrm{~mL})$, dried over $\mathrm{MgSO}_{4}$ and the sovlent removed under reduced pressure. The crude product was purified by flash chromatography on silica gel $(\mathrm{EtOAc} / n$-pentane, $1 / 2)$ and the pure target compound (3, $0.65 \mathrm{~g}, 1.19 \mathrm{mmol}, 74 \%)$ obtained as colorless foam.

TLC: $R_{\mathrm{f}}=0.67(\mathrm{EtOAc} / n$-pentane, $1 / 2)$.

${ }^{1} \mathbf{H}-\mathrm{NMR}\left(300 \mathrm{MHz}, \mathrm{CDCl}_{3}\right): \delta(\mathrm{ppm})=1.48(\mathrm{~s}, 9 \mathrm{H}, t-\mathrm{Bu}), 1.67\left(\mathrm{~s}, 6 \mathrm{H}, 3 \times \mathrm{Ad}-4^{\prime}-\right.$ $\left.\mathrm{H}_{2}\right), 1.98\left(\mathrm{~s}, 6 \mathrm{H}, 3 \times \mathrm{Ad}-2^{\prime}-\mathrm{H}_{2}\right), 2.07\left(\mathrm{~s}, 3 \mathrm{H}, 3 \times \mathrm{Ad}-3^{\prime}-\mathrm{H}\right), 2.71$ (ddd, J = $15.7 \mathrm{~Hz}$, $\left.J=4.6 \mathrm{~Hz}, J=3.9 \mathrm{~Hz}, 2 \mathrm{H}, \beta-\mathrm{H}_{2}\right), 4.23(\mathrm{t}, J=7.1 \mathrm{~Hz}, 1 \mathrm{H}$, Fmoc-9"-H), $4.28-4.49$ $\left(\mathrm{m}, 3 \mathrm{H}, \alpha-\mathrm{H}\right.$, Fmoc-10"- $\left.\mathrm{H}_{2}\right), 5.20(\mathrm{~s}, 1 \mathrm{H}, \mathrm{NH}), 6.16(\mathrm{~s}, 1 \mathrm{H}, \mathrm{NH}), 7.31(\mathrm{t}, J=7.4 \mathrm{~Hz}$, 2 H, Fmoc-2"-H, Fmoc-7"-H), 7.40 (t, J = 7.4 Hz, 2 H, Fmoc-3"-H, Fmoc-6"-H), 7.62 (d, $J=7.4$ Hz, 2 H, Fmoc-1"-H, Fmoc-8"-H), 7.76 (d, J = 7.4 Hz, 2 H, Fmoc-4"-H, Fmoc-5"-H).

$\operatorname{ESI-MS(+)~}(\mathrm{m} / \mathrm{z}(\%)): 545.3(12)[\mathrm{M}+\mathrm{H}]^{+}, 567.3(50)[\mathrm{M}+\mathrm{Na}]^{+}, 1089.5(16)[2 \mathrm{M}+\mathrm{H}]^{+}$, $1111.5(100)[2 \mathrm{M}+\mathrm{Na}]^{+}$. 


\section{HR-MS (ESI):}

calculated for $\left[\mathrm{C}_{33} \mathrm{H}_{41} \mathrm{~N}_{2} \mathrm{O}_{5}\right]^{+}\left([\mathrm{M}+\mathrm{H}]^{+}\right)$: 545.3010; found: 545.3006,

calculated for $\left[\mathrm{C}_{33} \mathrm{H}_{40} \mathrm{~N}_{2} \mathrm{O}_{5} \mathrm{Na}\right]^{+}\left([\mathrm{M}+\mathrm{Na}]^{+}\right)$: 567.2829; found: 567.2827 . 
(S)-2-(9-fluorenylmethyloxycarbonyl)-4-(1-adamantylamino)-4-oxobutanic acid (4)
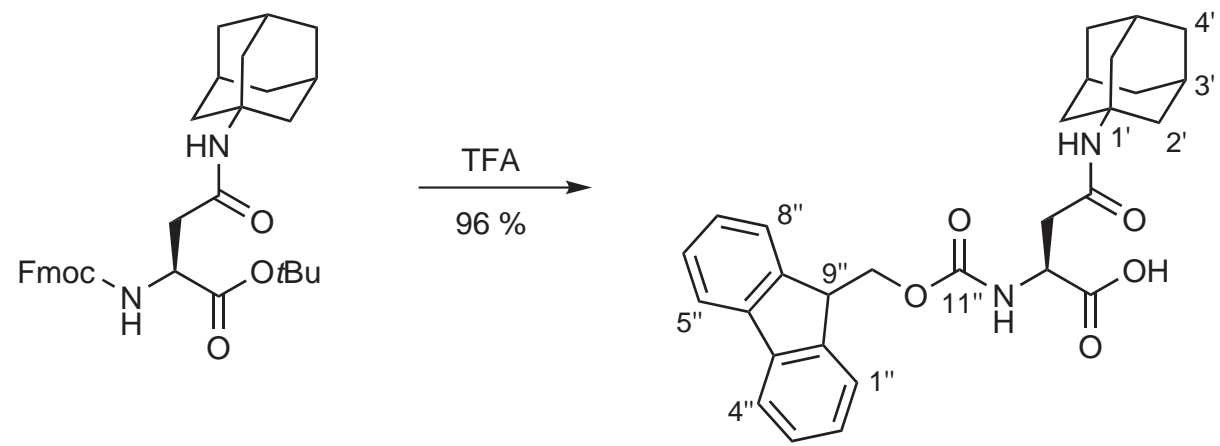

3

Fmoc-Asn(Ad)-OH (4)

$\mathrm{C}_{29} \mathrm{H}_{32} \mathrm{~N}_{2} \mathrm{O}_{5}$

$488.5 \mathrm{~g} / \mathrm{mol}$

Compound 3 ( $0.65 \mathrm{~g}, 1.19 \mathrm{mmol}, 1.00$ eq.) in TFA $/ \mathrm{H}_{2} \mathrm{O}(5 \mathrm{~mL}, 95 / 5, v / v)$ was stirred for $2 \mathrm{~h}$. The solvent was removed by nitrogen stream and residual TFA was coevaporated three times by toluene. The residue was dissolved in EtOAc $(50 \mathrm{~mL})$, washed with $\mathrm{NH}_{4} \mathrm{Cl}$ solution $(0.1 \mathrm{M}$, $3 \times 25 \mathrm{~mL}$ ), dried over $\mathrm{MgSO}_{4}$ and the solvent removed under reduced pressure. The crude product was purified by flash chromatography on silica gel (DCM/MeOH/AcOH, 90/10/0.5) and the pure target compound $(4,0.56 \mathrm{~g}, 1.15 \mathrm{mmol}, 96 \%)$ obtained as light brown foam.

TLC: $R_{\mathrm{f}}=0.28(\mathrm{DCM} / \mathrm{MeOH} / \mathrm{AcOH}, 90 / 10 / 0.5)$.

${ }^{1} \mathbf{H}-\mathbf{N M R}\left(300 \mathrm{MHz}, \mathrm{CDCl}_{3}\right): \delta(\mathrm{ppm})=1.68\left(\mathrm{~s}, 6 \mathrm{H}, 3 \times \mathrm{Ad}-4^{\prime}-\mathrm{H}_{2}\right), 2.00\left(\mathrm{~s}, 6 \mathrm{H}, 3 \times \mathrm{Ad}-2^{\prime}-\right.$ $\mathrm{H}_{2}$ ), $2.10\left(\mathrm{~s}, 3 \mathrm{H}, 3 \times \mathrm{Ad}^{-3}-\mathrm{H}\right), 2.70(\mathrm{dd}, J=15.7 \mathrm{~Hz}, J=9.4 \mathrm{~Hz}, 1 \mathrm{H}, \beta \mathrm{a}-\mathrm{H}), 2.85$ (dd, $J=15.7 \mathrm{~Hz}, J=2.3 \mathrm{~Hz}, 1 \mathrm{H}, \beta \mathrm{b}-\mathrm{H}) 4.21$ (t, J=7.1 Hz, $1 \mathrm{H}$, Fmoc-9"-H), 4.32 - 4.45 $\left(\mathrm{m}, 3 \mathrm{H}, 2-\mathrm{H}\right.$, Fmoc-10"- $\left.\mathrm{H}_{2}\right), 5.64\left(\mathrm{~s}_{\mathrm{br}}, 1 \mathrm{H}, \mathrm{OH}\right), 5.84(\mathrm{~s}, 1 \mathrm{H}, \mathrm{NH}), 6.14(\mathrm{~s}, 1 \mathrm{H}, \mathrm{NH}), 7.31$ $\left(\mathrm{t},{ }^{3} \mathrm{~J}_{\mathrm{HH}}=7.4 \mathrm{~Hz}, 2 \mathrm{H}\right.$, Fmoc-2"-H, Fmoc-7"-H ), 7.41 (t, J = 7.4 Hz, $2 \mathrm{H}$, Fmoc-3"-H, Fmoc-6"-H), 7.59 (d, J = 7.4 Hz, 2 H, Fmoc-1"-H, Fmoc-8"-H), 7.77 (d, J = 7.4 Hz, $2 \mathrm{H}$, Fmoc-4"-H, Fmoc-5"-H).

${ }^{13}$ C-NMR $\left(75 \mathrm{MHz}, \mathrm{CDCl}_{3}\right): \delta(\mathrm{ppm})=29.24$ (3 x Ad-C-3'), 36.07 (3 x Ad-C-4'), 28.67 (C- $\beta$ ), 41.10 (C- $\alpha$ ), 46.95 (Fmoc-C-9"), 50.75 (3 x Ad-C-2'), 53.13 (Ad-C-1'), 67.29 (Fmoc-C10"), 119.96 (Fmoc-C-4", Fmoc-C-5"), 125.09 (Fmoc-C-1", Fmoc-C-8"), 127.06 (Fmoc-C-2", Fmoc-C-7"), 127.72 (Fmoc-C-3", Fmoc-C-6"), 141.21 (Fmoc-C-4"a, Fmoc-C-4"b), 143.54 (Fmoc-C-8"a, Fmoc-C-9"a), 156.04 (Fmoc-C-11'), $171.06(\gamma-C O), 172.75\left(\mathrm{CO}_{2} \mathrm{H}\right)$.

ESI-MS(+) $(m / z(\%)): 489.2(52)[\mathrm{M}+\mathrm{H}]^{+}, 511.2(100)[\mathrm{M}+\mathrm{Na}]^{+}, 999.5(45)[2 \mathrm{M}+\mathrm{Na}]^{+}$, $1487.7(45)[3 \mathrm{M}+\mathrm{Na}]^{+}$. 
ESI-MS(-) $(m / z(\%)): 487.2(100)[M-H]^{-}, 975.4(23)[2 \mathrm{M}-\mathrm{H}]^{-}$.

\section{HR-MS (ESI):}

calculated for $\left[\mathrm{C}_{29} \mathrm{H}_{33} \mathrm{~N}_{2} \mathrm{O}_{5}\right]^{+}\left([\mathrm{M}+\mathrm{H}]^{+}\right)$: 489.2384; found: 489.2376, calculated for $\left[\mathrm{C}_{29} \mathrm{H}_{31} \mathrm{~N}_{2} \mathrm{O}_{5}\right]^{-}\left([\mathrm{M}-\mathrm{H}]^{-}\right)$: 487.2238; found: 487.2234, calculated for $\left[\mathrm{C}_{29} \mathrm{H}_{32} \mathrm{~N}_{2} \mathrm{O}_{5} \mathrm{Na}\right]^{+}\left([\mathrm{M}+\mathrm{Na}]^{+}\right)$: 511.2203; found: 511.2199 . 


\title{
8.6 Adamantyl modified Synaptobrevin-2 analogues ${ }^{[119]}$
}

\subsubsection{Synthesis of Synaptobrevin-2 (66 - 84) (5)}

\author{
5 \\ $\mathrm{C}_{82} \mathrm{H}_{135} \mathrm{~N}_{25} \mathrm{O}_{29}$ \\ $1935.1 \mathrm{~g} / \mathrm{mol}$
}

H-RADALQAGASQFETSAAKL-OH

Peptide 5 was synthesized according to GSP 2 (chapter 8.4.2) by automated solid phase peptide synthesis $(0.10 \mathrm{mmol})$ on a preloaded Fmoc-Leu-WANG resin $(0.30 \mathrm{mmol} / \mathrm{g})$ using the CEM Liberty 12 peptide synthesizer. The following amino acid derivatives were used:

Fmoc-Ala-OH, Fmoc-Arg(Pbf)-OH, Fmoc-Asp(OtBu)-OH, Fmoc-Gln(Trt)-OH, Fmoc-Gluc(OtBu)$\mathrm{OH}$, Fmoc-Gly-OH, Fmoc-Leu-OH, Fmoc-Lys(Boc)-OH, Fmoc-Phe-OH, Fmoc-Ser $(t \mathrm{Bu})-\mathrm{OH}$, Fmoc-Thr $(t \mathrm{Bu})-\mathrm{OH}$. According to GSP 3 (chapter 8.4.3) a small amount of peptide $\mathbf{5}$ was cleaved from the resin and completely deprotected for analysis by HPLC and mass spectrometry. The residual amount was used resin-bound for further syntheses (peptides $6-\mathbf{1 1}$ ).

HPLC (Macherey-Nagel NUCLEODUR ${ }^{\circledR}$ RP-C18 ec analytical column (100 Å, $\left.5 \mu \mathrm{m}, 250 \times 4.6 \mathrm{~mm}\right)$, gradient: $10-90 \% \mathrm{~B}$ in $30 \mathrm{~min}$ ): $t_{\mathrm{R} \text {, netto }}=11.78 \mathrm{~min}, t_{\mathrm{R} \text {, brutto }}=15.86 \mathrm{~min}$.

$\operatorname{ESI-MS(+)~}(\mathrm{m} / \mathrm{z}(\%)): 968.5(100)[\mathrm{M}+2 \mathrm{H}]^{2+}, 1936.0(3)[\mathrm{M}+\mathrm{H}]^{+}$.

ESI-MS(-) (m/z (\%)): $965.5(47)[\mathrm{M}-2 \mathrm{H}]^{2-}, 1934.0(12)[\mathrm{M}-\mathrm{H}]^{-}$.

HR-MS (ESI):

calculated for $\left[\mathrm{C}_{82} \mathrm{H}_{138} \mathrm{~N}_{25} \mathrm{O}_{29}\right]^{3+}\left([\mathrm{M}+3 \mathrm{H}]^{3+}\right)$ : 645.6692; found: 645.6705, calculated for $\left[\mathrm{C}_{82} \mathrm{H}_{133} \mathrm{~N}_{25} \mathrm{O}_{29}\right]^{2-}\left([\mathrm{M}-2 \mathrm{H}]^{2-}\right)$ : 965.9856; found: 965.9871 , calculated for $\left[\mathrm{C}_{82} \mathrm{H}_{137} \mathrm{~N}_{25} \mathrm{O}_{29}\right]^{2+}\left([\mathrm{M}+2 \mathrm{H}]^{2+}\right)$ : 968.0002; found: 968.0008, calculated for $\left[\mathrm{C}_{82} \mathrm{H}_{136} \mathrm{~N}_{25} \mathrm{O}_{29}\right]^{+}\left([\mathrm{M}+\mathrm{H}]^{+}\right)$: 1934.9930; found: 1934.9935, calculated for $\left[\mathrm{C}_{82} \mathrm{H}_{135} \mathrm{~N}_{25} \mathrm{O}_{29} \mathrm{Na}\right]^{+}\left([\mathrm{M}+\mathrm{Na}]^{+}\right)$: 1956.9750 ; found: 1956.9765 . 


\title{
8.6.2 Synthesis of Synaptobrevin-2 (28 - 84) (6)
}

H-SNRRLQQTQAQVDEVVDIMRVNVDKVLERDQKLSELDDRADALQAGASQFETSAAKL-OH

\author{
6 \\ $\mathrm{C}_{268} \mathrm{H}_{450} \mathrm{~N}_{84} \mathrm{O}_{95} \mathrm{~S}$ \\ $6401.0 \mathrm{~g} / \mathrm{mol}$
}

Peptide 6 was synthesized according to GSP 2 (chapter 8.4.2) by automated solid phase peptide synthesis $(0.10 \mathrm{mmol})$ on a preloaded Fmoc-Leu-WANG resin $(0.30 \mathrm{mmol} / \mathrm{g})$ using the CEM Liberty 12 peptide synthesizer. The synthesis was performed in two sections (Syb-2 (57 - 84) followed by Syb-2 $(28-56))$ due to reagent capacities of the peptide synthesizer. Between the two sections a small amount of peptide Syb-2 $57-84$ was cleaved from the resin and completely deprotected according to GSP 3 (chapter 8.4.3) for analysis by HPLC and mass spectrometry. The residual amount was used resin-bound for further synthesis of peptide $\mathbf{6}$. The following amino acid derivatives were used:

Fmoc-Ala-OH, Fmoc-Arg(Pbf)-OH, Fmoc-Asn(Trt)-OH, Fmoc-Asp(OtBu)-OH, Fmoc-Gln(Trt)$\mathrm{OH}$, Fmoc-Glu(OtBu)-OH, Fmoc-Gly-OH, Fmoc-lle-OH, Fmoc-Leu-OH, Fmoc-Lys(Boc)-OH, Fmoc-Met-OH, Fmoc-Phe-OH, Fmoc-Ser( $t \mathrm{Bu})-\mathrm{OH}$, Fmoc-Thr( $t \mathrm{Bu})-\mathrm{OH}$, Fmoc-Val-OH.

Alternatively, Peptide 6 was synthesized according to GSP 1 (chapter 8.4.1) by manual solid phase peptide synthesis $(0.05 \mathrm{mmol})$ on the preloaded resin $(250 \mathrm{mg})$ obtained from synthesis of peptide 5 (chapter 8.6.1). The following amino acid derivatives were used:

Fmoc-Ala-OH, Fmoc-Arg(Pbf)-OH, Fmoc-Asn(Trt)-OH, Fmoc-Asp(OtBu)-OH, Fmoc-Gln(Trt)$\mathrm{OH}$, Fmoc-Glu(OtBu)-OH, Fmoc-Gly-OH, Fmoc-lle-OH, Fmoc-Leu-OH, Fmoc-Lys(Boc)-OH, Fmoc-Met-OH, Fmoc-Ser( $t \mathrm{Bu})-\mathrm{OH}$, Fmoc-Thr( $t \mathrm{Bu})-\mathrm{OH}$, Fmoc-Val-OH.

Until amino acid D51 a coupling time of 30 min was applied except for R56, E55 (2 x 45 min) and V53 $(2 \times 30 \mathrm{~min})$. From amino acid V50 on coupling time was elongated to $2 \times 45 \mathrm{~min}$ except for R47 ( $2 \times 1 \mathrm{~h})$ and R31, R30 (3 $\times 1 \mathrm{~h})$. After coupling of ten amino acid residues a testcleavage was performed by using small amount of the resin and cleavage of the peptide according to GSP 3 (chapter 8.4.3). Synthesis progress was then analysed by mass spectrometry and analytical HPLC.

After complete synthesis peptide 6 was cleaved from resin and completely deprotected according to GSP 3 (chapter 8.4.3). After three times purification by HPLC peptide 6 (1.50 mg, $0.23 \mu \mathrm{mol}, 0.47 \%)$ was obtained.

HPLC (Macherey-Nagel NUCLEODUR ${ }^{\circledR}$ RP-C18 ec analytical column (100 A, $\left.5 \mu \mathrm{m}, 250 \times 4.6 \mathrm{~mm}\right)$, gradient: $40-50 \% \mathrm{~B}$ in $30 \mathrm{~min}$ ): $t_{\mathrm{R} \text {, netto }}=17.98 \mathrm{~min}, t_{\mathrm{R}, \text { brutto }}=22.52 \mathrm{~min}$.

ESI-MS(+) $(m / z(\%)): 915.3(100)[\mathrm{M}+7 \mathrm{H}]^{7+}, 1067.7(100)[\mathrm{M}+6 \mathrm{H}]^{6+}, 1281.1(24)$ 
$[\mathrm{M}+5 \mathrm{H}]^{5+}, 6397.3(100)[\mathrm{M}]$ (deconvoluted).

HR-MS (ESI):

calculated for $\left[\mathrm{C}_{268} \mathrm{H}_{457} \mathrm{~N}_{84} \mathrm{O}_{95} \mathrm{~S}\right]^{7+}\left([\mathrm{M}+7 \mathrm{H}]^{7+}\right)$ : 915.3330; found: 915.3325, calculated for $\left[\mathrm{C}_{268} \mathrm{H}_{456} \mathrm{~N}_{84} \mathrm{O}_{95} \mathrm{~S}\right]^{6+}\left([\mathrm{M}+6 \mathrm{H}]^{6+}\right)$ : 1067.7200; found: 1067.7221 , calculated for $\left[\mathrm{C}_{268} \mathrm{H}_{455} \mathrm{~N}_{84} \mathrm{O}_{95} \mathrm{~S}\right]^{5+}\left([\mathrm{M}+5 \mathrm{H}]^{5+}\right)$ : 1281.0626; found: 1281.0655, calculated for $\mathrm{C}_{268} \mathrm{H}_{450} \mathrm{~N}_{84} \mathrm{O}_{95} \mathrm{~S}$ [M]: 6397.3; found: 6397.3 (deconvoluted). 


\subsubsection{Synthesis of Synaptobrevin-2 (28 - 84) E55N(Ad), D65N(Ad)} (7)

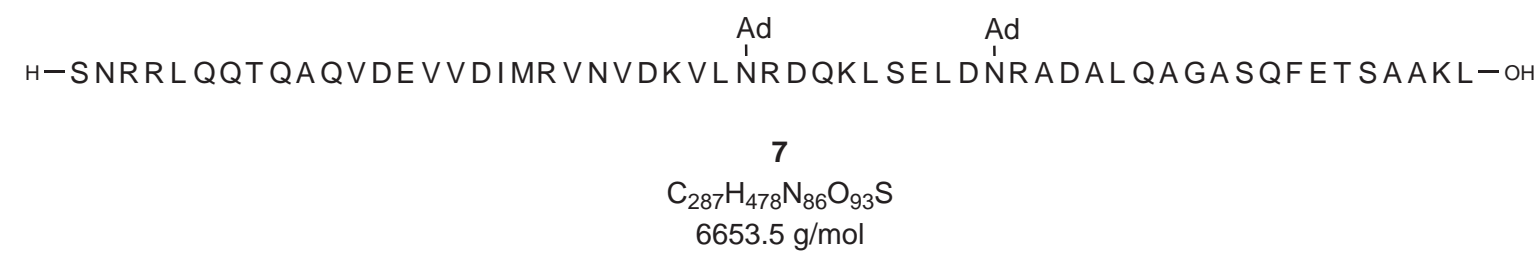

Peptide 7 was synthesized according to GSP 1 (chapter 8.4.1) by manual solid phase peptide synthesis $(0.05 \mathrm{mmol})$ on the preloaded resin $(225 \mathrm{mg})$ obtained from synthesis of peptide 5 (chapter 8.6.1). The following amino acid derivatives were used:

Fmoc-Ala-OH, Fmoc-Arg(Pbf)-OH, Fmoc-Asn(Trt)-OH, Fmoc-Asn(Ad)-OH (4), Fmoc-Asp(OtBu)-OH, Fmoc-Gln(Trt)-OH, Fmoc-Glu(OtBu)-OH, Fmoc-Gly-OH, Fmoc-lle-OH, FmocLeu-OH, Fmoc-Lys(Boc)-OH, Fmoc-Met-OH, Fmoc-Ser(tBu)-OH, Fmoc-Thr(tBu)-OH, Fmoc$\mathrm{Val}-\mathrm{OH}$.

Until amino acid D51 a coupling time of 30 min was applied except for R56 ( $2 \times 45 \mathrm{~min}$ ) and V53 $(2 \times 30 \mathrm{~min})$. From amino acid V50 on coupling time was elongated to $2 \times 45 \mathrm{~min}$ except for R47 $(2 \times 1 \mathrm{~h})$ and R31, R30 $(3 \times 1 \mathrm{~h})$. Coupling time for amino acid building block 4 was elongated to $2 \times 45 \mathrm{~min}$. After coupling of ten amino acid residues a testcleavage was performed by using a small amount of the resin and cleavage of the peptide according to GSP 3 (chapter 8.4.3). Synthesis progress was then analysed by mass spectrometry and analytical HPLC. After complete synthesis peptide 7 was cleaved from resin and completely deprotected according to GSP 3 (chapter 8.4.3). After three times purification by HPLC peptide 7 (1.37 mg, $0.21 \mu \mathrm{mol}, 0.41 \%$ ) was obtained.

HPLC (Macherey-Nagel NUCLEODUR ${ }^{\circledR}$ RP-C18 ec analytical column $(100 \AA$, $5 \mu \mathrm{m}, 250 \mathrm{x}$ $4.6 \mathrm{~mm}$ ), gradient: $45-55 \% \mathrm{~B}$ in $30 \mathrm{~min}): t_{\mathrm{R}, \text { netto }}=15.12 \mathrm{~min}, t_{\mathrm{R} \text {, brutto }}=19.36 \mathrm{~min}$.

ESI-MS(+) $(m / z(\%)): 832.57(18)[\mathrm{M}+8 \mathrm{H}]^{8+}, 951.37(100)[\mathrm{M}+7 \mathrm{H}]^{7+}, 1109.76(66)$ $[\mathrm{M}+6 \mathrm{H}]^{6+}, 1331.50(8)[\mathrm{M}+5 \mathrm{H}]^{5+}, 6652.52(100)[\mathrm{M}+\mathrm{H}]^{+}$.

\section{HR-MS (ESI):}

calculated for $\left[\mathrm{C}_{287} \mathrm{H}_{485} \mathrm{~N}_{86} \mathrm{O}_{93} \mathrm{~S}\right]^{7+}\left([\mathrm{M}+7 \mathrm{H}]^{7+}\right)$ : 951.3661; found: 951.3663, calculated for $\left[\mathrm{C}_{287} \mathrm{H}_{484} \mathrm{~N}_{86} \mathrm{O}_{93} \mathrm{~S}\right]^{6+}\left([\mathrm{M}+6 \mathrm{H}]^{6+}\right)$ : 1109.7593; found: 1109.7589, calculated for $\left[\mathrm{C}_{287} \mathrm{H}_{479} \mathrm{~N}_{86} \mathrm{O}_{93} \mathrm{~S}\right]^{+}\left([\mathrm{M}+\mathrm{H}]^{+}\right)$: 6652.51 ; found: 6652.52 . 


\title{
8.6.4 Synthesis of Synaptobrevin-2 (28 - 84) D51N(Ad), E55N(Ad)
}

(8)

\author{
Ad
$H-S N R R L Q Q T$
Ad
H. \\ 8 \\ $\mathrm{C}_{287} \mathrm{H}_{478} \mathrm{~N}_{86} \mathrm{O}_{93} \mathrm{~S}$ \\ $6653.5 \mathrm{~g} / \mathrm{mol}$
}

Peptide 8 was synthesized according to GSP 1 (chapter 8.4.1) by manual solid phase peptide synthesis $(0.05 \mathrm{mmol})$ on the preloaded resin $(250 \mathrm{mg})$ obtained from synthesis of peptide $\mathbf{5}$ (chapter 8.6.1). The following amino acid derivatives were used:

Fmoc-Ala-OH, Fmoc-Arg(Pbf)-OH, Fmoc-Asn(Trt)-OH, Fmoc-Asn(Ad)-OH (4), Fmoc-Asp(OtBu)-OH, Fmoc-Gln(Trt)-OH, Fmoc-Glu(OtBu)-OH, Fmoc-Gly-OH, Fmoc-lle-OH, FmocLeu-OH, Fmoc-Lys(Boc)-OH, Fmoc-Met-OH, Fmoc-Ser(tBu)-OH, Fmoc-Thr(tBu)-OH, Fmoc$\mathrm{Val}-\mathrm{OH}$.

Until amino acid D51 a coupling time of 30 min was applied except for R56 (2 x $45 \mathrm{~min}$ ) and V53 $(2 \times 30 \mathrm{~min})$. From amino acid V50 on coupling time was elongated to $2 \times 45 \mathrm{~min}$ except for R47 $(2 \times 1 \mathrm{~h})$ and R31, R30 $(3 \times 1 \mathrm{~h})$. Coupling time for amino acid building block 4 was elongated to $2 \times 45 \mathrm{~min}$. After coupling of ten amino acid residues a testcleavage was performed by using a small amount of the resin and cleavage of the peptide according to GSP 3 (chapter 8.4.3). Synthesis progress was then analysed by mass spectrometry and analytical HPLC. After complete synthesis peptide $\mathbf{8}$ was cleaved from resin and completely deprotected according to GSP 3 (chapter 8.4.3). After three times purification by HPLC peptide 8 (1.29 mg, $0.19 \mu \mathrm{mol}, 0.39 \%)$ was obtained.

HPLC (Macherey-Nagel NUCLEODUR ${ }^{\circledR}$ VarioPrep RP-C18 ec semipreperative column (100 $\AA$, $5 \mu \mathrm{m}, 250 \times 10.0 \mathrm{~mm}$ ), gradient: $45-55 \% \mathrm{~B}$ in $30 \mathrm{~min}): t_{\mathrm{R}, \text { netto }}=14.65 \mathrm{~min}, t_{\mathrm{R} \text {, brutto }}=20.51 \mathrm{~min}$.

$\operatorname{ESI-MS}(+)(m / z(\%)): 832.57(12)[\mathrm{M}+8 \mathrm{H}]^{8+}, 951.37(100)[\mathrm{M}+7 \mathrm{H}]^{7+}, 1109.76(82)$ $[\mathrm{M}+6 \mathrm{H}]^{6+}, 1331.51(12)[\mathrm{M}+5 \mathrm{H}]^{5+}, 1664.38(1)[\mathrm{M}+4 \mathrm{H}]^{4+}, 6652.53(100)[\mathrm{M}+\mathrm{H}]^{+}$.

HR-MS (ESI):

calculated for $\left[\mathrm{C}_{287} \mathrm{H}_{485} \mathrm{~N}_{86} \mathrm{O}_{93} \mathrm{~S}\right]^{7+}\left([\mathrm{M}+7 \mathrm{H}]^{7+}\right)$ : 951.3661; found: 951.3659, calculated for $\left[\mathrm{C}_{287} \mathrm{H}_{484} \mathrm{~N}_{86} \mathrm{O}_{93} \mathrm{~S}\right]^{6+}\left([\mathrm{M}+6 \mathrm{H}]^{6+}\right)$ : 1109.7593; found: 1109.7584, calculated for $\left[\mathrm{C}_{287} \mathrm{H}_{479} \mathrm{~N}_{86} \mathrm{O}_{93} \mathrm{~S}\right]^{+}\left([\mathrm{M}+\mathrm{H}]^{+}\right)$: 6652.51; found: 6652.53 . 


\subsubsection{Synthesis of Synaptobrevin-2 (28 - 84) D51N(Ad), E55N(Ad), D65N(Ad) (9)}

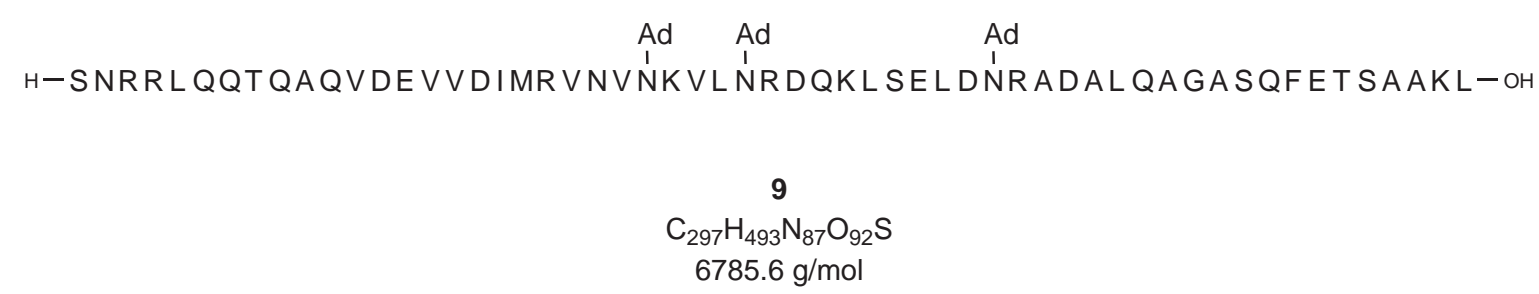

Peptide 9 was synthesized according to GSP 1 (chapter 8.4.1) by manual solid phase peptide synthesis $(0.05 \mathrm{mmol})$ on the preloaded resin $(250 \mathrm{mg})$ obtained from synthesis of peptide $\mathbf{5}$ (chapter 8.6.1). The following amino acid derivatives were used:

Fmoc-Ala-OH, Fmoc-Arg(Pbf)-OH, Fmoc-Asn(Trt)-OH, Fmoc-Asn(Ad)-OH (4), Fmoc-Asp(OtBu)-OH, Fmoc-Gln(Trt)-OH, Fmoc-Glu(OtBu)-OH, Fmoc-Gly-OH, Fmoc-lle-OH, FmocLeu-OH, Fmoc-Lys(Boc)-OH, Fmoc-Met-OH, Fmoc-Ser(tBu)-OH, Fmoc-Thr(tBu)-OH, Fmoc$\mathrm{Val}-\mathrm{OH}$.

Until amino acid D51 a coupling time of 30 min was applied except for R56 ( $2 \times 45 \mathrm{~min}$ ) and V53 $(2 \times 30 \mathrm{~min})$. From amino acid V50 on coupling time was elongated to $2 \times 45 \mathrm{~min}$ except for R47 $(2 \times 1 \mathrm{~h})$ and R31, R30 $(3 \times 1 \mathrm{~h})$. Coupling time for amino acid building block 4 was elongated to $2 \times 45 \mathrm{~min}$. After coupling of ten amino acid residues a testcleavage was performed by using a small amount of the resin and cleavage of the peptide according to GSP 3 (chapter 8.4.3). Synthesis progress was then analysed by mass spectrometry and analytical HPLC. After complete synthesis peptide $\mathbf{9}$ was cleaved from resin and completely deprotected according to GSP 3 (chapter 8.4.3). After three times purification by HPLC peptide $\mathbf{9}$ (1.09 mg, $0.16 \mu \mathrm{mol}, 0.32 \%$ ) was obtained.

HPLC (Macherey-Nagel NUCLEODUR ${ }^{\circledR}$ RP-C18 ec analytical column (100 A, $\left.5 \mu \mathrm{m}, 250 \times 4.6 \mathrm{~mm}\right)$, gradient: $45-55 \% \mathrm{~B}$ in $30 \mathrm{~min}$ ): $t_{\mathrm{R} \text {, netto }}=21.98 \mathrm{~min}, t_{\mathrm{R} \text {, brutto }}=26.34 \mathrm{~min}$.

ESI-MS(+) $(m / z(\%)): 849.22(28)[\mathrm{M}+8 \mathrm{H}]^{8+}, 970.39(100)[\mathrm{M}+7 \mathrm{H}]^{7+}, 1131.95(50)$ $[\mathrm{M}+6 \mathrm{H}]^{6+}, 1358.14(7)[\mathrm{M}+5 \mathrm{H}]^{5+}, 1697.41(1)[\mathrm{M}+4 \mathrm{H}]^{4+}, 6785.65(100)[\mathrm{M}+\mathrm{H}]^{+}$.

HR-MS (ESI):

calculated for $\left[\mathrm{C}_{297} \mathrm{H}_{501} \mathrm{~N}_{87} \mathrm{O}_{92} \mathrm{~S}\right]^{8+}\left([\mathrm{M}+8 \mathrm{H}]^{8+}\right)$ : 849.2120; found: 849.2119, calculated for $\left[\mathrm{C}_{297} \mathrm{H}_{500} \mathrm{~N}_{87} \mathrm{O}_{92} \mathrm{~S}\right]^{7+}\left([\mathrm{M}+7 \mathrm{H}]^{7+}\right): 970.3841$; found: 970.3842 , calculated for $\left[\mathrm{C}_{297} \mathrm{H}_{499} \mathrm{~N}_{87} \mathrm{O}_{92} \mathrm{~S}\right]^{6+}\left([\mathrm{M}+6 \mathrm{H}]^{6+}\right)$ : 1131.9469 ; found: 1131.9465, calculated for $\left[\mathrm{C}_{297} \mathrm{H}_{493} \mathrm{~N}_{87} \mathrm{O}_{92} \mathrm{~S}\right]^{+}\left([\mathrm{M}+\mathrm{H}]^{+}\right)$: 6785.64; found: 6785.65 . 


\title{
8.6.6 Synthesis of Synaptobrevin-2 (28 - 84) Q58N(Ad) (10)
}

\author{
Ad \\ H-SNRRLQQTQAQVDEVVDIMRVNVDKVLERDNंKLSELDDRADALQAGASQFETSAAKL-OH \\ 10 \\ $\mathrm{C}_{277} \mathrm{H}_{462} \mathrm{~N}_{84} \mathrm{O}_{95} \mathrm{~S}$ \\ $6520.4 \mathrm{~g} / \mathrm{mol}$
}

Peptide 10 was synthesized according to GSP 1 (chapter 8.4 .1 ) by manual solid phase peptide synthesis $(0.05 \mathrm{mmol})$ on the preloaded resin $(250 \mathrm{mg})$ obtained from synthesis of peptide $\mathbf{5}$ (chapter 8.6.1). The following amino acid derivatives were used:

Fmoc-Ala-OH, Fmoc-Arg(Pbf)-OH, Fmoc-Asn(Trt)-OH, Fmoc-Asn(Ad)-OH (4), Fmoc-Asp(OtBu)-OH, Fmoc-Gln(Trt)-OH, Fmoc-Glu(OtBu)-OH, Fmoc-Gly-OH, Fmoc-lle-OH, FmocLeu-OH, Fmoc-Lys(Boc)-OH, Fmoc-Met-OH, Fmoc-Ser(tBu)-OH, Fmoc-Thr(tBu)-OH, Fmoc$\mathrm{Val}-\mathrm{OH}$.

Until amino acid D51 a coupling time of 30 min was applied except for R56, E55 (2 x 45 min) and V53 ( $2 \times 30 \mathrm{~min})$. From amino acid V50 on coupling time was elongated to $2 \times 45 \mathrm{~min}$ except for R47 $(2 \times 1 \mathrm{~h})$ and R31, R30 $(3 \times 1 \mathrm{~h})$. Coupling time for amino acid building block 4 was elongated to $2 \times 45 \mathrm{~min}$. After coupling of ten amino acid residues a testcleavage was performed by using a small amount of the resin and cleavage of the peptide according to GSP 3 (chapter 8.4.3). Synthesis progress was then analysed by mass spectrometry and analytical HPLC. After complete synthesis peptide $\mathbf{1 0}$ was cleaved from resin and completely deprotected according to GSP 3 (chapter 8.4.3). After three times purification by HPLC peptide $10(1.47 \mathrm{mg}, 0.23 \mu \mathrm{mol}, 0.45 \%)$ was obtained.

HPLC (Macherey-Nagel NUCLEODUR ${ }^{\circledR}$ RP-C18 ec analytical column (100 $\left.,, 5 \mu \mathrm{m}, 250 \times 4.6 \mathrm{~mm}\right)$, gradient: $45-55 \% \mathrm{~B}$ in $30 \mathrm{~min}): t_{\mathrm{R}, \text { netto }}=14.19 \mathrm{~min}, t_{\mathrm{R}, \text { brutto }}=18.55 \mathrm{~min}$.

ESI-MS(+) $(m / z(\%)): 816.06(9)[\mathrm{M}+8 \mathrm{H}]^{8+}, 932.49(100)[\mathrm{M}+7 \mathrm{H}]^{7+}, 1087.74(73)$ $[\mathrm{M}+6 \mathrm{H}]^{6+}, 1305.07(12)[\mathrm{M}+5 \mathrm{H}]^{5+}, 6520.39(100)[\mathrm{M}+\mathrm{H}]^{+}$.

\section{HR-MS (ESI):}

calculated for $\left[\mathrm{C}_{277} \mathrm{H}_{470} \mathrm{~N}_{84} \mathrm{O}_{95} \mathrm{~S}\right]^{8+}\left([\mathrm{M}+8 \mathrm{H}]^{8+}\right)$ : 816.0536; found: 816.0542,

calculated for $\left[\mathrm{C}_{277} \mathrm{H}_{469} \mathrm{~N}_{84} \mathrm{O}_{95} \mathrm{~S}\right]^{7+}\left([\mathrm{M}+7 \mathrm{H}]^{7+}\right)$ : 932.4888; found: 932.4886,

calculated for $\left[\mathrm{C}_{277} \mathrm{H}_{468} \mathrm{~N}_{84} \mathrm{O}_{95} \mathrm{~S}\right]^{6+}\left([\mathrm{M}+6 \mathrm{H}]^{6+}\right)$ : 1087.7357; found: 1087.7357,

calculated for $\left[\mathrm{C}_{277} \mathrm{H}_{462} \mathrm{~N}_{84} \mathrm{O}_{95} \mathrm{~S}\right]^{+}\left([\mathrm{M}+\mathrm{H}]^{+}\right)$: 6520.37; found: 6520.39 . 


\title{
8.6.7 Synthesis of Synaptobrevin-2 (28 - 84) E62N(Ad) (11)
}

$\mathrm{Ad}$

H-SNRRLQQTQAQVDEVVDIMRVNVDKVLERDQKLSN่LDDRADALQAGASQFETSAAKL-OH

\author{
11 \\ $\mathrm{C}_{277} \mathrm{H}_{462} \mathrm{~N}_{84} \mathrm{O}_{95} \mathrm{~S}$ \\ $6520.4 \mathrm{~g} / \mathrm{mol}$
}

Peptide 11 was synthesized according to GSP 1 (chapter 8.4.1) by manual solid phase peptide synthesis $(0.05 \mathrm{mmol})$ on the preloaded resin $(225 \mathrm{mg})$ obtained from synthesis of peptide $\mathbf{5}$ (chapter 8.6.1). The following amino acid derivatives were used:

Fmoc-Ala-OH, Fmoc-Arg(Pbf)-OH, Fmoc-Asn(Trt)-OH, Fmoc-Asn(Ad)-OH (4), Fmoc-Asp(OtBu)-OH, Fmoc-Gln(Trt)-OH, Fmoc-Glu(OtBu)-OH, Fmoc-Gly-OH, Fmoc-lle-OH, FmocLeu-OH, Fmoc-Lys(Boc)-OH, Fmoc-Met-OH, Fmoc-Ser( $t \mathrm{Bu})-\mathrm{OH}$, Fmoc-Thr( $t \mathrm{Bu})-\mathrm{OH}$, Fmoc$\mathrm{Val}-\mathrm{OH}$.

Until amino acid D51 a coupling time of 30 min was applied except for R56, E55 (2 x 45 min) and V53 ( $2 \times 30 \mathrm{~min})$. From amino acid V50 on coupling time was elongated to $2 \times 45 \mathrm{~min}$ except for R47 $(2 \times 1 \mathrm{~h})$ and R31, R30 ( $3 \times 1 \mathrm{~h})$. Coupling time for amino acid building block 4 was elongated to $2 \times 45 \mathrm{~min}$. After coupling of ten amino acid residues a testcleavage was performed by using a small amount of the resin and cleavage of the peptide according to GSP 3 (chapter 8.4.3). Synthesis progress was then analysed by mass spectrometry and analytical HPLC. After complete synthesis peptide $\mathbf{1 1}$ was cleaved from resin and completely deprotected according to GSP 3 (chapter 8.4.3). After three times purification by HPLC peptide 11 (1.63 mg, $0.25 \mu \mathrm{mol}, 0.50 \%)$ was obtained.

HPLC (Macherey-Nagel NUCLEODUR ${ }^{\circledR}$ RP-C18 ec analytical column (100 $\left.\AA, 5 \mu \mathrm{m}, 250 \times 4.6 \mathrm{~mm}\right)$, gradient: $45-55 \% \mathrm{~B}$ in $30 \mathrm{~min}$ ): $t_{\mathrm{R} \text {,netto }}=11.15 \mathrm{~min}, t_{\mathrm{R} \text {, brutto }}=14.69 \mathrm{~min}$.

ESI-MS(+) $(m / z(\%)): 815.93(15)[\mathrm{M}+8 \mathrm{H}]^{8+}, 932.35(100)[\mathrm{M}+7 \mathrm{H}]^{7+}, 1087.57(78)$ $[\mathrm{M}+6 \mathrm{H}]^{6+}, 1304.88(16)[\mathrm{M}+5 \mathrm{H}]^{5+}, 6519.40(100)[\mathrm{M}+\mathrm{H}]^{+}$.

\section{HR-MS (ESI):}

calculated for $\left[\mathrm{C}_{277} \mathrm{H}_{470} \mathrm{~N}_{85} \mathrm{O}_{94} \mathrm{~S}\right]^{7+}\left([\mathrm{M}+7 \mathrm{H}]^{7+}\right)$ : 932.3482; found: 932.3484, calculated for $\left[\mathrm{C}_{277} \mathrm{H}_{469} \mathrm{~N}_{85} \mathrm{O}_{94} \mathrm{~S}\right]^{6+}\left([\mathrm{M}+6 \mathrm{H}]^{6+}\right)$ : 1087.5717 ; found: 1087.5720 , calculated for $\left[\mathrm{C}_{277} \mathrm{H}_{463} \mathrm{~N}_{85} \mathrm{O}_{94} \mathrm{~S}\right]^{+}\left([\mathrm{M}+\mathrm{H}]^{+}\right)$: 6519.39; found: 6519.40 . 


\subsection{Photocleavable Amino Acid Building Blocks}

\subsubsection{Synthesis of caged lysine $N$-Fmoc buidling block (16) ${ }^{[132,137]}$}

Synthesis of 4,5-Dimethoxy-2-nitrobenzyl-4'-nitrophenylcarbonate (14) ${ }^{[137]}$<smiles>COc1cc(CO)c([N+](=O)[O-])cc1OC</smiles>

12<smiles>O=C(Cl)Oc1ccc([N+](=O)[O-])cc1</smiles>

13<smiles>COc1cc(COC(=O)Oc2ccc([N+](=O)[O-])cc2)c([N+](=O)[O-])cc1OC</smiles>

14

$\mathrm{C}_{16} \mathrm{H}_{14} \mathrm{~N}_{2} \mathrm{O}_{9}$ $378.2 \mathrm{~g} / \mathrm{mol}$

4,5-Dimethoxy-2-nitrobenzylalcohol (12, $5.03 \mathrm{~g}, 23.6 \mathrm{mmol}, 1.00 \mathrm{eq}$.$) and p-nitrophenylchloro-$ formate $(13,9.43 \mathrm{~g}, 47.0 \mathrm{mmol}, 2.00$ eq. $)$ were suspended in dry DCM (50 mL). After addition of DIPEA ( $8.35 \mathrm{~mL}, 47.0 \mathrm{mmol}, 2.00$ eq.) the reaction mixture became homogeneous and was stirred overnight at room temperature. The solvent was removed under reduced pressure and the residue was recrystallized from $\mathrm{EtOH}$, filtrated and washed with hot $\mathrm{EtOH}(2 \times 10 \mathrm{~mL})$. Drying under reduced pressure yielded the target compound (14, $4.95 \mathrm{~g}, 13.1 \mathrm{mmol}, 55 \%)$ as a light yellow solid.

${ }^{1} \mathbf{H}-\mathrm{NMR}\left(300 \mathrm{MHz}, \mathrm{CDCl}_{3}\right): \delta(\mathrm{ppm})=3.98(\mathrm{~s}, 3 \mathrm{H}, \mathrm{OMe}), 4.02(\mathrm{~s}, 3 \mathrm{H}, \mathrm{OMe}), 5.71$ (s, $\left.2 \mathrm{H}, 7-\mathrm{H}_{2}\right), 7.01(\mathrm{~s}, 1 \mathrm{H}, 6-\mathrm{H}), 7.41\left(\mathrm{~d}, J=9.3 \mathrm{~Hz}, 2 \mathrm{H}, 2^{\prime}-\mathrm{H}, 6^{\prime}-\mathrm{H}\right), 7.77(\mathrm{~s}, 1 \mathrm{H}, 3-\mathrm{H}), 8.29$ $\left(\mathrm{d}, J=9.3 \mathrm{~Hz}, 2 \mathrm{H}, 3^{\prime}-\mathrm{H}, 5^{\prime}-\mathrm{H}\right)$.

${ }^{13} \mathrm{C}-\mathrm{NMR}\left(125 \mathrm{MHz}, \mathrm{CDCl}_{3}\right): \delta(\mathrm{ppm})=56.84(\mathrm{OMe}), 56.93$ (OMe), 68.04 (C-7), 108.77 (C-3), 111.01 (C-6), 122.06 (C-2', C-6'), 125.42 (C-1), 125.72 (C-3', C-5'), 140.38 (C-2), 145.89 (C-4'), 149.17 (C-4), 152.41 (C=O), 154.04 (C-5), 155.69 (C-1').

ESI-MS(+) $(\mathrm{m} / z(\%)): 396.1(100)\left[\mathrm{M}+\mathrm{NH}_{4}\right]^{+}, 401.1(88)[\mathrm{M}+\mathrm{Na}]^{+}, 417.0(12)[\mathrm{M}+\mathrm{K}]^{+}$, $779.1(62)[2 \mathrm{M}+\mathrm{Na}]^{+}$.

HR-MS (ESI):

calculated for $\left[\mathrm{C}_{16} \mathrm{H}_{18} \mathrm{~N}_{3} \mathrm{O}_{9}\right]^{+}\left(\left[\mathrm{M}+\mathrm{NH}_{4}\right]^{+}\right)$: 396.1038; found: 396.1041, calculated for $\left[\mathrm{C}_{16} \mathrm{H}_{14} \mathrm{~N}_{2} \mathrm{O}_{9} \mathrm{Na}\right]^{+}\left([\mathrm{M}+\mathrm{Na}]^{+}\right)$: 401.0592; found: 401.0593, calculated for $\left[\mathrm{C}_{16} \mathrm{H}_{14} \mathrm{~N}_{2} \mathrm{O}_{9} \mathrm{~K}\right]^{+}\left([\mathrm{M}+\mathrm{K}]^{+}\right)$: 417.0331; found: 417.0328 . 


\section{Synthesis of Fmoc-Lys(Nvoc)-OH (16)}<smiles>COc1cc(COC(=O)Oc2ccc([N+](=O)[O-])cc2)c([N+](=O)[O-])cc1OC</smiles>

14
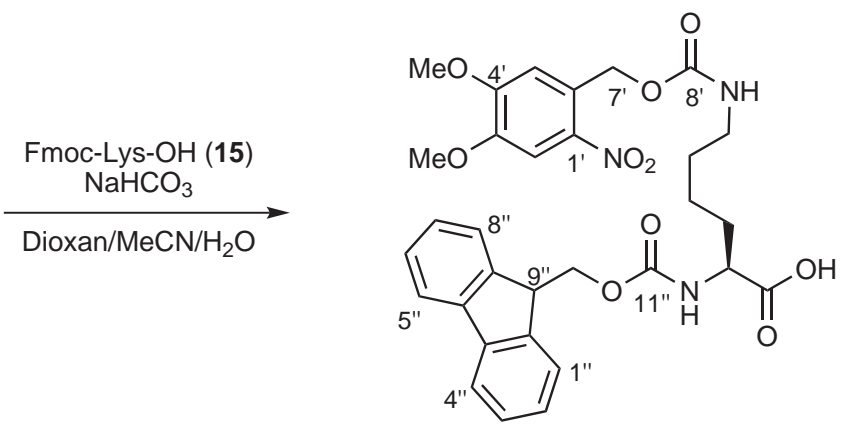

Fmoc-Lys(Nvoc)-OH (16)

$\mathrm{C}_{31} \mathrm{H}_{33} \mathrm{~N}_{3} \mathrm{O}_{10}$

$607.2 \mathrm{~g} / \mathrm{mol}$

Fmoc-Lys-OH (15, $1.95 \mathrm{~g}, 5.29 \mathrm{mmol}, 1.00$ eq.) was suspended in $\mathrm{H}_{2} \mathrm{O} / 1$,4-dioxane/MeCN $(1 / 1 / 1,45 \mathrm{~mL})$ and $\mathrm{Na}_{2} \mathrm{CO}_{3}(0.56 \mathrm{~g}, 5.29 \mathrm{mmol}, 1.00$ eq. $)$ was added. Afterwards, additional amount of $\mathrm{H}_{2} \mathrm{O}(10 \mathrm{~mL})$ and $\mathrm{MeCN}(5 \mathrm{~mL})$ were added to transfer all solids into solution. 4,5-Dimethoxy-2-nitrobenzyl-4'-nitrophenylcarbonate $(\mathbf{1 4}, 2.00 \mathrm{~g}, 5.29 \mathrm{mmol}, 1.00 \mathrm{eq}$.) was suspended in 1,4-dioxane $(25 \mathrm{~mL})$ and added drop-wise to the reaction mixture. After addition the mixture was stirred under exclusion of light at room temperature overnight. The solvent was removed under reduced pressure and the residue was dissolved in aqueous $\mathrm{NaHSO}_{4}$ solution $(1 \mathrm{M}, 75 \mathrm{~mL})$ and $\mathrm{DCM}(125 \mathrm{~mL})$. The phases were seperated and the aqueous phase was extracted with DCM $(125 \mathrm{~mL})$. The combined organic layers were dried over $\mathrm{MgSO}_{4}$ and the solvent was removed under reduced pressure. Purification by flash chromatography on silica gel ( $n$-pentane/EtOAc, $1 / 1 \rightarrow \mathrm{DCM} / \mathrm{MeOH} / \mathrm{AcOH}, 95 / 5 / 0.5)$ yielded the target compound $(\mathbf{1 6}, 2.43 \mathrm{~g}, 4.00 \mathrm{mmol}, 76 \%)$ as a yellow solid.

TLC: $R_{\mathrm{f}}=0.47(\mathrm{DCM} / \mathrm{MeOH} / \mathrm{AcOH}, 95 / 5 / 0.5)$.

${ }^{1} \mathbf{H}-\mathrm{NMR}\left(300 \mathrm{MHz}, \mathrm{dmso}_{6}\right): \delta(\mathrm{ppm})=1.26-1.53\left(\mathrm{~m}, 4 \mathrm{H}, \gamma-\mathrm{H}_{2}, \delta-\mathrm{H}_{2}\right), 1.53-$ $1.80\left(\mathrm{~m}, 2 \mathrm{H}, \beta-\mathrm{H}_{2}\right), 3.03\left(\mathrm{dd}, J=12.1,6.2 \mathrm{~Hz}, 2 \mathrm{H}, \epsilon-\mathrm{H}_{2}\right), 3.85\left(\mathrm{~s}, 3 \mathrm{H}, 3{ }^{\prime}-\mathrm{OMe}\right), 3.88$ (s, $\left.3 \mathrm{H}, 4^{\prime}-\mathrm{OMe}\right), 3.90-4.00(\mathrm{~m}, 1 \mathrm{H}, \alpha-\mathrm{H}), 4.17-4.25\left(\mathrm{~m}, 1 \mathrm{H}, 9^{\prime \prime}-\mathrm{H}\right), 4.25-4.32(\mathrm{~m}, 2 \mathrm{H}$, $\left.10 "-\mathrm{H}_{2}\right), 5.33\left(\mathrm{~s}, 2 \mathrm{H}, 7^{\prime}-\mathrm{H}_{2}\right), 7.16\left(\mathrm{~s}, 1 \mathrm{H}, 5^{\prime}-\mathrm{H}\right), 7.31$ (t, J = 7.4 Hz, $\left.2 \mathrm{H}, 2^{\prime \prime}-\mathrm{H}, 7{ }^{\prime \prime}-\mathrm{H}\right), 7.40$ (t, $\left.J=7.4 \mathrm{~Hz}, 3 \mathrm{H}, 3^{\prime \prime}-\mathrm{H}, 6^{\prime \prime}-\mathrm{H}, \epsilon-\mathrm{NH}\right), 7.56(\mathrm{~d}, J=8.0 \mathrm{~Hz}, 1 \mathrm{H}, \alpha-\mathrm{NH}), 7.67\left(\mathrm{~s}, 1 \mathrm{H}, 2^{\prime}-\mathrm{H}\right), 7.71$ $\left(\mathrm{d}, J=7.4 \mathrm{~Hz}, 2 \mathrm{H}, 1^{\prime \prime}-\mathrm{H}, 8 "-\mathrm{H}\right), 7.86(\mathrm{~d}, J=7.4 \mathrm{~Hz}, 2 \mathrm{H}, 4 "-\mathrm{H}, 5 "-\mathrm{H}), 12.51\left(\mathrm{~s}_{\mathrm{br}}, 1 \mathrm{H}, \mathrm{CO}_{2} \mathrm{H}\right)$.

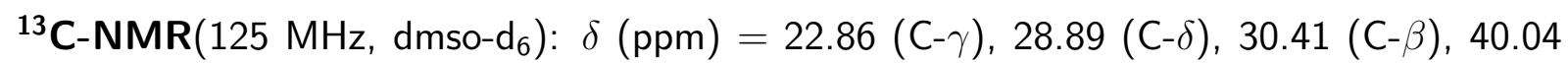
(C- $\epsilon$ ), 46.65 (C-9"), 53.74(C- $\alpha$ ), 56.01 (OMe), 56.12 (OMe), 62.16 (C-7'), 65.56 (C-10"), 108.13 (C-2'), 110.52 (C-5'), 119.98 (C-4", C-5"), 125.16 (C-1", C-8"), 126.96 (C-2", C-7"), 127.53 (C-3", C-6"), 128.11 (C-6'), 139.27 (C-1'), 140.64 (C-4"a, C-4"b), 143.75 (C-8"a, 
C-9"a), 147.67 (C-3'), 153.30 (C-4'), 155.63 (C-8'), 156.11 (C-11"), $173.85\left(\mathrm{CO}_{2} \mathrm{H}\right)$.

ESI-MS(+) $(\mathrm{m} / \mathrm{z}(\%)): 608.2(3)[\mathrm{M}+\mathrm{H}]^{+}, 630.2(90)[\mathrm{M}+\mathrm{Na}]^{+}, 646.2(100)[\mathrm{M}+\mathrm{K}]^{+}$, $1237.4(13)[2 \mathrm{M}+\mathrm{Na}]^{+}, 1253.4(8)[2 \mathrm{M}+\mathrm{K}]^{+}$.

$\operatorname{ESI-MS}(-)(m / z(\%)): 606.2(100)[\mathrm{M}-\mathrm{H}]^{-}$.

\section{HR-MS (ESI):}

calculated for $\left[\mathrm{C}_{31} \mathrm{H}_{32} \mathrm{~N}_{3} \mathrm{O}_{10}\right]^{-}\left([\mathrm{M}-\mathrm{H}]^{-}\right): 606.2093$; found: 606.2095, calculated for $\left[\mathrm{C}_{31} \mathrm{H}_{34} \mathrm{~N}_{3} \mathrm{O}_{10}\right]^{+}\left([\mathrm{M}+\mathrm{H}]^{+}\right)$: 608.2239; found: 608.2231, calculated for $\left[\mathrm{C}_{31} \mathrm{H}_{33} \mathrm{~N}_{3} \mathrm{O}_{10} \mathrm{Na}\right]^{+}\left([\mathrm{M}+\mathrm{Na}]^{+}\right)$: 630.2058; found: 630.2054.

calculated for $\left[\mathrm{C}_{31} \mathrm{H}_{33} \mathrm{~N}_{3} \mathrm{O}_{10} \mathrm{~K}\right]^{+}\left([\mathrm{M}+\mathrm{K}]^{+}\right)$: 646.1798; found: 646.1795 . 


\subsubsection{Synthesis of caged arginine $N$-Fmoc building block (21) [134, 138]}

Synthesis of Imino(methylthio)methyl carbamic acid-6-nitroveratryl ester (18)<smiles>COc1cc(COC(=O)Oc2ccc([N+](=O)[O-])cc2)c([N+](=O)[O-])cc1OC</smiles>

14<smiles>CC(=N)NC(C)(C)C</smiles>

17<smiles>COc1cc(COC(=O)NC(=N)S(C)(=O)=O)c([N+](=O)[O-])cc1OC</smiles>

18

$\mathrm{C}_{12} \mathrm{H}_{15} \mathrm{~N}_{3} \mathrm{O}_{6} \mathrm{~S}$

$329.3 \mathrm{~g} / \mathrm{mol}$

S-methylisothiourea semisulfate $(\mathbf{1 7}, 2.59 \mathrm{~g}, 18.6 \mathrm{mmol}, 2.00 \mathrm{eq}$.$) was suspended in DCM$ $(26 \mathrm{~mL})$. After addition of aqueous sodium hydroxide solution $(4 \mathrm{M}, 2.60 \mathrm{~mL}, 10.4 \mathrm{mmol}$, 1.12 eq.), a clear solution was obtained. A suspension of 4,5-dimethoxy-2-nitrobenzyl-4'nitrophenylcarbonate $(\mathbf{1 4}, 3.52 \mathrm{~g}, 9.31 \mathrm{mmol}, 1.00$ eq. $)$ in $\mathrm{DCM}(34 \mathrm{~mL})$ was added at $0{ }^{\circ} \mathrm{C}$. The reaction mixture was stirred overnight and was allowed to warm slowly to room temperature. Afterwards, $\mathrm{H}_{2} \mathrm{O}(10 \mathrm{~mL})$ was added and the layers were separated. The aqueous layer was extracted with DCM $(3 \times 50 \mathrm{~mL})$ and the combined organic layers were washed with hydrochloric acid $(0.1 \mathrm{M}, 2 \times 50 \mathrm{~mL}), \mathrm{H}_{2} \mathrm{O}(3 \times 50 \mathrm{~mL})$ and saturated aqueous sodium chloride solution $(100 \mathrm{~mL})$. The organic layer was dried over $\mathrm{MgSO}_{4}$ and the solvent was removed under reduced pressure. After purification by flash chromatography on silica gel ( $n$-pentane/EtOAc, $3 / 1 \rightarrow$ $1 / 1 \rightarrow 3 / 7)$ the target compound $(\mathbf{1 8}, 1.85 \mathrm{~g}, 5.63 \mathrm{mmol}, 60 \%)$ was obtained as a yellow solid.

${ }^{1} \mathbf{H}-\mathbf{N M R}\left(300 \mathrm{MHz}, \mathrm{dmso}_{6}\right): \delta(\mathrm{ppm})=2.34\left(\mathrm{~s}, 3 \mathrm{H}, \mathrm{SCH}_{3}\right), 3.87(\mathrm{~s}, 3 \mathrm{H}, \mathrm{OMe})$, $3.88(\mathrm{~s}, 3 \mathrm{H}, \mathrm{OMe}), 5.33\left(\mathrm{~s}, 2 \mathrm{H}, 7-\mathrm{H}_{2}\right), 7.17(\mathrm{~s}, 1 \mathrm{H}, 6-\mathrm{H}), 7.68(\mathrm{~s}, 1 \mathrm{H}, 3-\mathrm{H}), 8.69\left(\mathrm{~s}_{\mathrm{br}}, 2 \mathrm{H}\right.$, $\mathrm{NH})$.

${ }^{13} \mathrm{C}-\mathrm{NMR}\left(125 \mathrm{MHz}, \mathrm{dmso}-\mathrm{d}_{6}\right): \delta(\mathrm{ppm})=13.07\left(\mathrm{SCH}_{3}\right), 56.11(\mathrm{OMe}), 56.18(\mathrm{OMe})$, 63.18 (C-7), 108.16 (C-3), 111.29 (C-6), 127.33 (C-1), 139.73 (C-2), 147.84 (C-4), 153.17 $(\mathrm{C}-5), 160.42(\mathrm{C}=\mathrm{N}), 173.18(\mathrm{C}=\mathrm{O})$.

ESI-MS(+) $(\mathrm{m} / z(\%)): 330.1(80)[\mathrm{M}+\mathrm{H}]^{+}, 352.1(76)[\mathrm{M}+\mathrm{Na}]^{+}, 368.1(100)[\mathrm{M}+\mathrm{K}]^{+}$, $659.2(7)[2 \mathrm{M}+\mathrm{H}]^{+}, 681.1(39)[2 \mathrm{M}+\mathrm{Na}]^{+}$.

ESI-MS(-) $(m / z(\%)): 280.1(100)\left[\mathrm{M}-\mathrm{H}_{2} \mathrm{SMe}^{-}, 328.1(19) \mathrm{M}-\mathrm{H}\right]^{-}$. 


\section{HR-MS (ESI):}

calculated for $\left[\mathrm{C}_{12} \mathrm{H}_{14} \mathrm{~N}_{3} \mathrm{O}_{6} \mathrm{~S}\right]^{-}\left([\mathrm{M}-\mathrm{H}]^{-}\right): 328.0609$; found: 328.0602,

calculated for $\left[\mathrm{C}_{12} \mathrm{H}_{16} \mathrm{~N}_{3} \mathrm{O}_{6} \mathrm{~S}\right]^{+}\left([\mathrm{M}+\mathrm{H}]^{+}\right)$: 330.0754; found: 330.0752 ,

calculated for $\left[\mathrm{C}_{12} \mathrm{H}_{15} \mathrm{~N}_{3} \mathrm{O}_{6} \mathrm{SNa}\right]^{+}\left([\mathrm{M}+\mathrm{Na}]^{+}\right)$: 352.0574; found: 352.0573 .

calculated for $\left[\mathrm{C}_{12} \mathrm{H}_{15} \mathrm{~N}_{3} \mathrm{O}_{6} \mathrm{SK}\right]^{+}\left([\mathrm{M}+\mathrm{K}]^{+}\right)$: 368.0313; found: 368.0313 . 


\section{Synthesis of Fmoc-Orn-OH (20)}

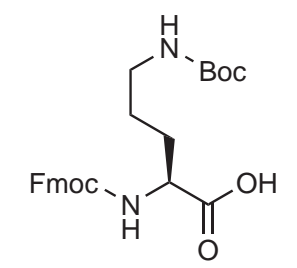

Fmoc-Orn(Boc)-OH (19)

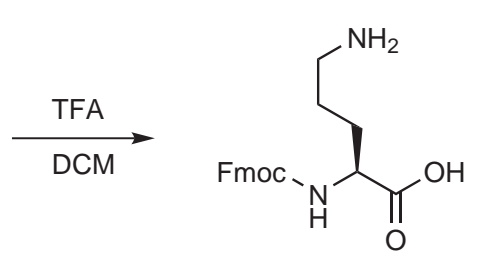

Fmoc-Orn-OH (20)

$\mathrm{C}_{20} \mathrm{H}_{22} \mathrm{~N}_{2} \mathrm{O}_{4}$

$354.4 \mathrm{~g} / \mathrm{mol}$

(TFA salt: $\mathrm{C}_{22} \mathrm{H}_{23} \mathrm{~F}_{3} \mathrm{~N}_{2} \mathrm{O}_{6}$

$468.2 \mathrm{~g} / \mathrm{mol}$ )

Fmoc-Orn(Boc)-OH (19, $5.00 \mathrm{~g}, 11.0 \mathrm{mmol}, 1.00$ eq.) was dissolved in TFA/DCM (1/1, $120 \mathrm{~mL}$ ) and stirred for $2 \mathrm{~h}$ at room temperature. Afterwards, the solvent was removed by nitrogen stream and the residue was co-evaporated with $\mathrm{Et}_{2} \mathrm{O}(5 \times 30 \mathrm{~mL})$ and toluene $(2 \times 30 \mathrm{~mL})$ under reduced pressure. Suspension in $\mathrm{H}_{2} \mathrm{O} / \mathrm{MeCN}(10 / 1,100 \mathrm{~mL})$ and lyophilisation yielded the target compound $(\mathbf{2 0}, 5.10 \mathrm{~g}, 10.9 \mathrm{mmol}, 99 \%)$ as colorless solid.

${ }^{1} \mathbf{H}-\mathbf{N M R}\left(300 \mathrm{MHz}\right.$, dmso-d $\left._{6}\right): \delta(\mathrm{ppm})=1.53-1.72\left(\mathrm{~m}, 3 \mathrm{H}, \beta-\mathrm{H}_{\mathrm{a}}, \gamma-\mathrm{H}_{2}\right), 1.72-$ $1.89\left(\mathrm{~m}, 1 \mathrm{H}, \beta-\mathrm{H}_{\mathrm{b}}\right), 2.73-2.88\left(\mathrm{~m}, 2 \mathrm{H}, \delta-\mathrm{H}_{2}\right), 3.91-4.02(\mathrm{~m}, 1 \mathrm{H}, \alpha-\mathrm{H}), 4.18-4.37(\mathrm{~m}$, $\left.3 \mathrm{H}, 9^{\prime}-\mathrm{H}, 10^{\prime}-\mathrm{H}_{2}\right), 7.33\left(\mathrm{t}, J=7.4 \mathrm{~Hz}, 2 \mathrm{H}, 2^{\prime}-\mathrm{H}, 7^{\prime}-\mathrm{H}\right), 7.41\left(\mathrm{t}, J=7.4 \mathrm{~Hz}, 2 \mathrm{H}, 3^{\prime}-\mathrm{H}\right.$, $\left.6^{\prime}-\mathrm{H}\right), 7.64(\mathrm{~d}, J=8.1 \mathrm{~Hz}, 1 \mathrm{H}, \alpha-\mathrm{NH}), 7.72\left(\mathrm{~d}, J=7.4 \mathrm{~Hz}, 2 \mathrm{H}, 1^{\prime}-\mathrm{H}, 8^{\prime}-\mathrm{H}\right), 7.79\left(\mathrm{~s}_{\mathrm{br}}, 2 \mathrm{H}\right.$, $\left.\delta-\mathrm{NH}_{2}\right), 7.89\left(\mathrm{~d}, J=7.4 \mathrm{~Hz}, 2 \mathrm{H}, 4^{\prime}-\mathrm{H}, 5^{\prime}-\mathrm{H}\right), 12.89\left(\mathrm{~s}_{\mathrm{br}}, 1 \mathrm{H}, \mathrm{CO}_{2} \mathrm{H}\right)$.

${ }^{13}$ C-NMR(125 MHz, dmso-d 6 ): $\delta(\mathrm{ppm})=23.88(\mathrm{C}-\gamma), 27.69(\mathrm{C}-\beta), 38.45(\mathrm{C}-\delta), 46.62$ (C-9'), 53.33 (C- $\alpha), 65.62$ (C-10'), 120.03 (C-4', C-5'), 125.16 (C-1', C-8'), 127.01 (C-2', C-7'), 127.57 (C-3', C-6'), 140.66 (C-4'a, C-4'b), 143.73 (C-8'a, C-9'a), 156.11 (C-11'), 173.38 $\left(\mathrm{CO}_{2} \mathrm{H}\right)$.

ESI-MS(+) $(m / z(\%)): 179.1(33)[\mathrm{M}+2 \mathrm{H}]^{2+}, 355.2(100)[\mathrm{M}+\mathrm{H}]^{+}, 393.1(5)[\mathrm{M}+\mathrm{K}]^{+}$, $709.4(2)[2 \mathrm{M}+\mathrm{H}]^{+}$.

ESI-MS(-) $(m / z(\%)): 467.2(89)[\mathrm{M}+\mathrm{TFA}-\mathrm{H}]^{-}$.

HR-MS (ESI):

calculated for $\left[\mathrm{C}_{20} \mathrm{H}_{23} \mathrm{~N}_{2} \mathrm{O}_{4}\right]^{+}\left([\mathrm{M}+\mathrm{H}]^{+}\right)$: 355.1652; found: 355.1654,

calculated for $\left[\mathrm{C}_{22} \mathrm{H}_{23} \mathrm{~F}_{3} \mathrm{~N}_{2} \mathrm{O}_{6}\right]^{-}\left([\mathrm{M}+\mathrm{TFA}-\mathrm{H}]^{-}\right)$: 467.1435; found: 467.1436. 


\section{Synthesis of Fmoc-Arg(Nvoc)-OH (21)}<smiles>COc1cc(COC(=O)NC(=N)S(C)(C)C)c([N+](=O)[O-])cc1OC</smiles>

18<smiles>NCCC[C@H](NC(F)F)C(=O)O</smiles>

Fmoc-Orn-OH·TFA (20)
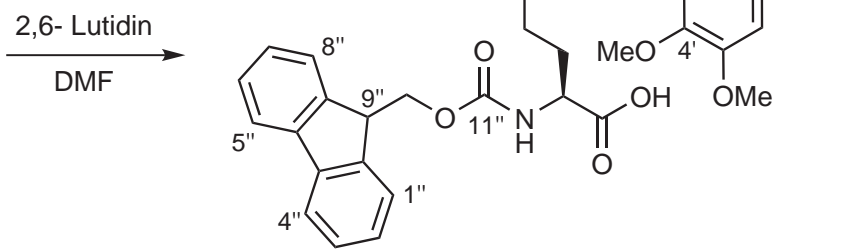

Fmoc-Arg(Nvoc)-OH (21)

$\mathrm{C}_{31} \mathrm{H}_{33} \mathrm{~N}_{5} \mathrm{O}_{10}$ $635.2 \mathrm{~g} / \mathrm{mol}$

Imino(methylthio)methyl carbamic acid-6-nitroveratryl ester (18, $4.05 \mathrm{~g}, 12.3 \mathrm{mmol}, 2.00$ eq.) and Fmoc-Orn-OH.TFA (20, $2.88 \mathrm{~g}, 6.15 \mathrm{mmol}, 1.00$ eq.) were dissolved in dry DMF $(70 \mathrm{~mL})$ and 2,6-lutidine $(7.14 \mathrm{~mL}, 6.59 \mathrm{~g}, 61.5 \mathrm{mmol}, 10.0 \mathrm{eq}$.) was slowly added at room temperature. The reaction mixture was stirred under light exclusion at room temperature overnight. The resulted precipitate was filtered and the filtrate concentrated under reduced pressure. Purification by flash chromatography on silical gel ( $n$-pentane/EtOAc, $1 / 2 \rightarrow \mathrm{DCM} / \mathrm{MeOH} / \mathrm{A}-$ $\mathrm{cOH}, 10 / 1 / 0.5)$ yielded the target compound $(\mathbf{2 1}, 1.35 \mathrm{~g}, 2.12 \mathrm{mmol}, 35 \%)$ as light yellow solid.

TLC: $R_{\mathrm{f}}=0.37(\mathrm{DCM} / \mathrm{MeOH} / \mathrm{AcOH}, 10 / 1 / 0.5)$.

${ }^{1} \mathbf{H}-N M R\left(300 \mathrm{MHz}\right.$, dmso-d $\left._{6}\right): \delta(\mathrm{ppm})=1.39-1.68\left(\mathrm{~m}, 3 \mathrm{H}, \beta-\mathrm{H}_{\mathrm{a}}, \gamma-\mathrm{H}_{2}\right), 1.68-$ $1.83\left(\mathrm{~m}, 1 \mathrm{H}, \beta-\mathrm{H}_{\mathrm{b}}\right), 3.07-3.19\left(\mathrm{~m}, 2 \mathrm{H}, \epsilon-\mathrm{H}_{2}\right), 3.84\left(\mathrm{~s}, 3 \mathrm{H}, 3^{\prime}-\mathrm{OMe}\right), 3.85$ (s, $\left.3 \mathrm{H}, 4{ }^{\prime}-\mathrm{OMe}\right)$, $3.87-3.95(\mathrm{~m}, 1 \mathrm{H}, \alpha-\mathrm{H}), 4.16-4.37\left(\mathrm{~m}, 3 \mathrm{H}, 9^{\prime \prime}-\mathrm{H}, 10^{\prime \prime}-\mathrm{H}_{2}\right), 5.29\left(\mathrm{~s}, 2 \mathrm{H}, 7^{\prime}-\mathrm{H}_{2}\right), 7.16$ (s,

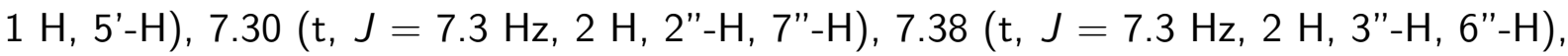
$7.64\left(\mathrm{~s}, 1 \mathrm{H}, 2^{\prime}-\mathrm{H}\right), 7.69$ (d, J = 7.3 Hz, $2 \mathrm{H}, 1^{\prime \prime}-\mathrm{H}, 8$ "'-H), 7.84 (d, J = 7.3 Hz, 2 H, 4"-H, 5"-H), $8.33\left(\mathrm{~s}_{\mathrm{br}}, 1 \mathrm{H}, \mathrm{CO}_{2} \mathrm{H}\right)$.

${ }^{13} \mathrm{C}-\mathrm{NMR}\left(125 \mathrm{MHz}, \mathrm{dmso}_{6}\right): \delta(\mathrm{ppm})=25.25(\mathrm{C}-\gamma), 29.12(\mathrm{C}-\beta), 39.75(\mathrm{C}-\epsilon), 46.79$ (C-9"), 54.65 (C- $\alpha$ ), 56.05 (OMe), 56.10 (OMe), 62.43 (C-7'), 65.47 (C-10"), 108.06 (C-2'), 110.39 (C-5'), 120.04 (C-4", C-5"), 125.18, 125.23 (C-1", C-8"), 127.04, 127.26 (C-2", C-7"), 127.58 (C-3", C-6"), 128.75 (C-6'), 139.36 (C-1'), 140.73 (C-4a", C-4b"), 143.83, 143.95, (C-8a", C-8b"), 147.56 (C-4'), 153.31 (C-3'), 158.10, 158.35 (C-11', C=NH), 161.41 (C-8').

ESI-MS(+) $(\mathrm{m} / \mathrm{z}(\%)): 636.2(100)[\mathrm{M}+\mathrm{H}]^{+}, 658.2(63)[\mathrm{M}+\mathrm{Na}]^{+}, 674.2(57)[\mathrm{M}+\mathrm{K}]^{+}$, $1293.4(7)[2 \mathrm{M}+\mathrm{Na}]^{+}$. 
ESI-MS(-) $(\mathrm{m} / \mathrm{z}(\%)): 634.2(100)[\mathrm{M}-\mathrm{H}]^{-}, 748.2(11)[\mathrm{M}+\mathrm{TFA}-\mathrm{H}]^{-}$.

HR-MS (ESI):

calculated for $\left[\mathrm{C}_{31} \mathrm{H}_{32} \mathrm{~N}_{5} \mathrm{O}_{10}\right]^{-}\left([\mathrm{M}-\mathrm{H}]^{-}\right)$: 634.2155; found: 634.2132, calculated for $\left[\mathrm{C}_{31} \mathrm{H}_{34} \mathrm{~N}_{5} \mathrm{O}_{10}\right]^{+}\left([\mathrm{M}+\mathrm{H}]^{+}\right)$: 636.2300; found: 636.2306, calculated for $\left[\mathrm{C}_{31} \mathrm{H}_{33} \mathrm{~N}_{5} \mathrm{O}_{10} \mathrm{Na}\right]^{+}\left([\mathrm{M}+\mathrm{Na}]^{+}\right)$: 658.2120; found: 658.2129. 


\subsubsection{Synthesis of caged aspartic acid $\mathbf{N}$-Fmoc building block (27) [107, 135]}

Synthesis of 3-(3,4-dimethoxyphenyl)-butan-2-one (23) [107, 135]<smiles>COc1ccc(CC(C)=O)cc1OC</smiles>

22

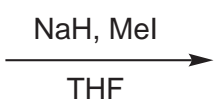

$\mathrm{C}_{12} \mathrm{H}_{16} \mathrm{O}_{3}$

$208.2 \mathrm{~g} / \mathrm{mol}$

3,4-Dimethoxyphenylacetone (22, $1.87 \mathrm{~mL}, 2.09 \mathrm{~g}, 10.8 \mathrm{mmol}, 1.00$ eq.) was added drop wise to a suspension of sodium hydride ( $60 \%$ in parrafin oil, $0.43 \mathrm{~g}, 10.8 \mathrm{mmol}, 1.00 \mathrm{eq}$.) in dry THF (25 mL) and stirred for $30 \mathrm{~min}$ at room temperature. The solution was cooled to $0{ }^{\circ} \mathrm{C}$, methyl iodide $(0.80 \mathrm{~mL}, 1.82 \mathrm{~g}, 12.8 \mathrm{mmol}, 1.19$ eq. $)$ added and afterwards stirred for $30 \mathrm{~min}$ at $0{ }^{\circ} \mathrm{C}$. The cooling bath was removed and the reaction mixture was stirred for $1 \mathrm{~h}$ at room temperature. The reaction was quenched by addition of sat. $\mathrm{NaHCO}_{3}$ solution $(100 \mathrm{~mL})$ and the aqueous layer was extracted with EtOAc $(3 \times 10 \mathrm{~mL})$. The combined organic layers were washed with saturated aqueous $\mathrm{NaCl}$ solution $\left(100 \mathrm{~mL}\right.$ ) and dried over $\mathrm{MgSO}_{4}$. The solvent was removed under reduced pressure to obtain the target compound $(23,2.22 \mathrm{~g}, 10.6 \mathrm{mmol}$, $99 \%$ ) as a brown oil. It was used for further synthesis without purification.

TLC: $R_{\mathrm{f}}=0.45(n$-pentane/EtOAc (5/1)).

${ }^{1} \mathbf{H}-\mathbf{N M R}\left(300 \mathrm{MHz}, \mathrm{CDCl}_{3}\right): \delta(\mathrm{ppm})=1.36\left(\mathrm{~d}, J=7.0 \mathrm{~Hz}, 3 \mathrm{H}, 4-\mathrm{H}_{3}\right), 2.05(\mathrm{~s}, 3 \mathrm{H}$, $\left.1-\mathrm{H}_{3}\right), 3.68(\mathrm{q}, J=7.0 \mathrm{~Hz}, 1 \mathrm{H}, 3-\mathrm{H}), 3.86(\mathrm{~s}, 6 \mathrm{H}, 2 \times \mathrm{OMe}), 6.69\left(\mathrm{~d}, J=1.9 \mathrm{~Hz}, 2^{\prime}-\mathrm{H}\right)$, $6.72-6.85\left(\mathrm{~m}, 2 \mathrm{H}, 5^{\prime}-\mathrm{H}, 6^{\prime}-\mathrm{H}\right)$.

ESI-MS(+) $(\mathrm{m} / \mathrm{z}(\%)): 209.2(100)[\mathrm{M}+\mathrm{H}]^{+}, 231.2(54)[\mathrm{M}+\mathrm{Na}]^{+}, 247.2(34)[\mathrm{M}+\mathrm{K}]^{+}$.

\section{HR-MS (ESI):}

calculated for $\left[\mathrm{C}_{12} \mathrm{H}_{17} \mathrm{O}_{3}\right]^{+}\left([\mathrm{M}+\mathrm{H}]^{+}\right)$: 209.1631; found: 209.1627,

calculated for $\left[\mathrm{C}_{12} \mathrm{H}_{16} \mathrm{O}_{3} \mathrm{Na}\right]^{+}\left([\mathrm{M}+\mathrm{Na}]^{+}\right)$: 231.1529; found: 231.1532 . 


\section{Synthesis of 3-(3,4-dimethoxyphenyl)-butan-2-ol (24) 107 135]}<smiles>COc1ccc(C(C)C(C)=O)cc1OC</smiles>

23

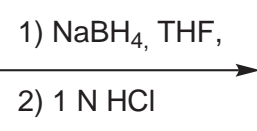<smiles>COc1ccc(C(C)C(C)O)cc1OC</smiles>

24

$\mathrm{C}_{12} \mathrm{H}_{18} \mathrm{O}_{3}$

$210.2 \mathrm{~g} / \mathrm{mol}$

Sodium borohydride $(0.90 \mathrm{~g}, 23.8 \mathrm{mmol}, 2.50 \mathrm{eq}$. $)$ was added at room temperature to a solution of 3-(3,4-dimethoxyphenyl)-butan-2-one (23, $2.00 \mathrm{~g}, 9.61 \mathrm{mmol}, 1.00 \mathrm{eq}$.) in THF/2-propanol $(30 \mathrm{~mL}, 1 / 2(v / v))$. The reaction mixture was stirred for $2 \mathrm{~h}$ at room temperature and afterwards unreacted sodium borohydride was quenched with $1 \mathrm{M} \mathrm{HCl}$ at $0{ }^{\circ} \mathrm{C} . \mathrm{H}_{2} \mathrm{O}(200 \mathrm{~mL})$ and $\mathrm{EtOAc}(100 \mathrm{~mL})$ were added and the aqueous layer was extracted with $\mathrm{EtOAc}(100 \mathrm{~mL})$. The combined organic layers were washed with $\mathrm{H}_{2} \mathrm{O}(150 \mathrm{~mL})$ and saturated aqueous $\mathrm{NaCl}$ solution $(50 \mathrm{~mL})$, dried over $\mathrm{MgSO}_{4}$ and the solvent was removed under reduced pressure. Purification by flash chromatography on silica gel ( $n$-pentane/acetone, $5 / 1$ ) yielded the target compound $(24,1.28 \mathrm{~g}, 6.09 \mathrm{mmol}, 63 \%)$ as an erythro-threo $(1 / 2)$ mixture of an light yellow solid.

TLC: $R_{\mathrm{f}}=0.54(n$-pentane/acetone, $4 / 1)$.

${ }^{1} \mathbf{H}-\mathbf{N M R}\left(500 \mathrm{MHz}, \mathrm{CDCl}_{3}\right): \delta(\mathrm{ppm})=1.08\left(\mathrm{~d}, \mathrm{~J}=6.2 \mathrm{~Hz}, 1 \mathrm{H}, 3-\mathrm{CH}_{3}\right.$ (erythro)), $1.22\left(\mathrm{~d}, J=6.2 \mathrm{~Hz}, 2 \mathrm{H}, 3-\mathrm{CH}_{2}\right.$ (threo)), 1.24 (d, J = 7.1 Hz, $2 \mathrm{H}, 1-\mathrm{H}_{3}$ (threo)), 1.29 (d, $J=7.1 \mathrm{~Hz}, 1 \mathrm{H}, 1-\mathrm{H}_{3}$ (erythro)), $1.50\left(\mathrm{~s}_{\mathrm{br}}, 1 \mathrm{H}, \mathrm{OH}\right.$ ), 2.60 (quin, $J=7.1 \mathrm{~Hz}, 0.7 \mathrm{H}, 2-\mathrm{H}$ (threo)), 2.68 (quin, $J=7.1 \mathrm{~Hz}, 0.3 \mathrm{H}, 2-\mathrm{H}$ (erythro)), $3.75-3.82(\mathrm{~m}, 0.7 \mathrm{H}, 3-\mathrm{H}$ (threo)), $3.81-3.85(\mathrm{~m}, 0.3 \mathrm{H}, 3-\mathrm{H}$ (erythro)), $3.85(\mathrm{~s}, 1 \mathrm{H}, \mathrm{OMe}$ (erythro)), $3.86(\mathrm{~s}, 2 \mathrm{H}, \mathrm{OMe}($ threo $))$, 3.87 (s, $1 \mathrm{H}, \mathrm{OMe}$ (erythro)), 3.87 (s, $2 \mathrm{H}, \mathrm{OMe}$ (threo)), $6.71-6.85\left(\mathrm{~m}, 3 \mathrm{H}, 2^{\prime}-\mathrm{H}, 5^{\prime}-\mathrm{H}, 6^{\prime}-\mathrm{H}\right)$.

${ }^{13}$ C-NMR $\left(125 \mathrm{MHz}, \mathrm{CDCl}_{3}\right): \delta(\mathrm{ppm})=15.91$ (C-4), $20.56(\mathrm{C}-1), 47.64(\mathrm{C}-3), 55.83,55.86$ (OMe), 72.40 (C-2), 111.16 (C-2'), 111.35 (C-5'), 119.80 (C-6'), 136.05 (C-1'), 147.80 (C-4'), 148.99 (C-3').

ESI-MS(+) $(m / z(\%)): 193.1(100)[\mathrm{M}-\mathrm{OH}]^{+}, 211.6(6)[\mathrm{M}+\mathrm{H}]^{+}, 233.1(90)[\mathrm{M}+\mathrm{Na}]^{+}$, $249.1(38)[\mathrm{M}+\mathrm{K}]^{+}$. 


\section{HR-MS (ESI):}

calculated for $\left[\mathrm{C}_{12} \mathrm{H}_{18} \mathrm{O}_{3} \mathrm{Na}\right]^{+}\left([\mathrm{M}+\mathrm{Na}]^{+}\right)$: 233.1148; found: 233.1154,

calculated for $\left[\mathrm{C}_{12} \mathrm{H}_{18} \mathrm{O}_{3} \mathrm{~K}\right]^{+}\left([\mathrm{M}+\mathrm{K}]^{+}\right)$: 249.0888; found: 249.0886 . 


\section{Synthesis of 3-(4,5-dimethoxy-2-nitrophenyl)-butan-2-ol (25) 107] 135]}<smiles>COc1ccc(C(C)C(C)O)cc1OC</smiles>

24

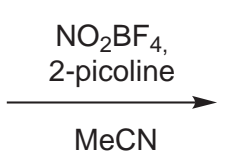

25

$255.2 \mathrm{~g} / \mathrm{mol}$

Nitronium tetrafluoroborate $(1.09 \mathrm{~g}, 8.22 \mathrm{mmol}, 1.40 \mathrm{eq}$.$) was dissolved in dry acetonitrile$ $(15 \mathrm{~mL})$ at $-30{ }^{\circ} \mathrm{C}, 2$-picoline $(0.87 \mathrm{~mL}, 0.82 \mathrm{~g}, 8.81 \mathrm{mmol}, 1.50$ eq. $)$ was added drop-wise and the mixture was stirred for $30 \mathrm{~min}$ at $-30{ }^{\circ} \mathrm{C}$. A solution of 3-(3,4-dimethoxyphenyl)-butan-2-ol $(24,1.23 \mathrm{~g}, 5.87 \mathrm{mmol}, 1.00 \mathrm{eq}$. $)$ in dry acetonitrile $(10 \mathrm{~mL})$ was slowly added and the reaction mixture was stirred for $1 \mathrm{~h}$ at $-30{ }^{\circ} \mathrm{C}$ and allowed to warm up to room temperature overnight. The reaction was quenched by addition of sat. $\mathrm{NaHCO}_{3}$ solution $(100 \mathrm{~mL})$ and the aqueous layer was extracted with EtOAc $(2 \times 100 \mathrm{~mL})$. The combined organic layers were washed with $\mathrm{H}_{2} \mathrm{O}(100 \mathrm{~mL})$, saturated aqueous $\mathrm{NaCl}$ solution $(50 \mathrm{~mL})$, dried over $\mathrm{MgSO}_{4}$ and the solvent was removed under reduced pressure. Purification by flash chromatography on silica gel ( $n$-pentane/acetone, 4/1) yielded the target compound $(25,0.56 \mathrm{~g}, 2.19 \mathrm{mmol}$, $37 \%)$ as an erythro/threo (1/2) mixture of an light yellow solid.

TLC: $R_{\mathrm{f}}=0.59(n$-pentane/acetone, $4 / 1)$.

${ }^{1} \mathbf{H}-\mathbf{N M R}\left(500 \mathrm{MHz}, \mathrm{CDCl}_{3}\right): \delta(\mathrm{ppm})=1.18\left(\mathrm{~d}, J=6.2 \mathrm{~Hz}, 1 \mathrm{H}, 3-\mathrm{CH}_{3}\right.$ (erythro)), $1.24\left(\mathrm{~d}, J=6.2 \mathrm{~Hz}, 2 \mathrm{H}, 3-\mathrm{CH}_{2}\right.$ (threo)), $1.30\left(\mathrm{~d}, J=7.0 \mathrm{~Hz}, 2 \mathrm{H}, 1-\mathrm{H}_{3}\right.$ (threo)), 1.33 (d, J = 7.0 Hz, $1 \mathrm{H}, 1-\mathrm{H}_{3}$ (erythro)), $1.69\left(\mathrm{~s}_{\mathrm{br}}, 1 \mathrm{H}, \mathrm{OH}\right), 3.53(\mathrm{p}, J=7.0 \mathrm{~Hz}, 1 \mathrm{H}, 2-\mathrm{H})$, $3.85-3.94(\mathrm{~m}, 1 \mathrm{H}, 3-\mathrm{H}), 3.91(\mathrm{~s}, 3 \mathrm{H}, \mathrm{OMe}), 3.95$ (s, $3 \mathrm{H}, \mathrm{OMe}), 6.92\left(\mathrm{~s}, 0.3 \mathrm{H}, 66^{\prime}-\mathrm{H}\right.$ (erythro)), $6.96(\mathrm{~s}, 0.7 \mathrm{H}, 6$ ' $-\mathrm{H}($ threo $)), 7.38\left(\mathrm{~s}, 0.7 \mathrm{H}, 3^{\prime}-\mathrm{H}\right.$ (threo)), 7.41 (s, $0.3 \mathrm{H}, 3^{\prime}-\mathrm{H}$ (erythro)).

${ }^{13} \mathrm{C}-\mathrm{NMR}\left(125 \mathrm{MHz}, \mathrm{CDCl}_{3}\right): \delta(\mathrm{ppm})=15.02$ (C-4), 21.57 (C-1), 40.77 (C-3), 56.23 (OMe), 72.33 (C-2), 107.64 (C-3'), 109.72 (C-6'), 133.28 (C-1'), 143.18 (C-2'), 147.02 (C-4'), $152.70\left(\mathrm{C}-5^{\prime}\right)$.

ESI-MS(+) $(m / z(\%)): 256.1(2)[\mathrm{M}+\mathrm{H}]^{+}, 278.1(66)[\mathrm{M}+\mathrm{Na}]^{+}, 533.2(10)[2 \mathrm{M}+\mathrm{Na}]^{+}$. 
HR-MS (ESI):

calculated for $\left[\mathrm{C}_{12} \mathrm{H}_{17} \mathrm{NO}_{5} \mathrm{Na}\right]^{+}\left([\mathrm{M}+\mathrm{Na}]^{+}\right)$: 278.0999; found: 278.0999 . 


\section{Synthesis of $N$-Fmoc- $\delta$-[3-(4,5-dimethoxy-2-nitrophenyl)-2-butyl]-L-aspartic acid tert- butyl ester (Fmoc-Asp(DMNPB)-OtBu (26) $)^{[135}$}<smiles>COc1cc([C@H](C)C(C)O)c([N+](=O)[O-])cc1OC</smiles>

25<smiles>CCOC(=O)[C@H](CC(=O)O)NC(F)F</smiles>

1

Fmoc-Asp-OtBu<smiles>COC(=O)[C@H](CC(=O)O[C@H](I)[C@@H](C)c1cc(OC)[14c](OC)cc1[N+](=O)[O-])NC(F)F</smiles>

26

$\mathrm{C}_{35} \mathrm{H}_{40} \mathrm{~N}_{2} \mathrm{O}_{10}$ $648.7 \mathrm{~g} / \mathrm{mol}$

3-(4,5-dimethoxy-2-nitrophenyl)-butan-2-ol (25, $0.56 \mathrm{~g}, 2.19 \mathrm{mmol}, 1.00 \mathrm{eq}$.$) was dissolved$ in dry DCM $(20 \mathrm{~mL})$ and Fmoc-Asp-OtBu (1, $0.90 \mathrm{~g}, 2.19 \mathrm{mmol}, 1.00$ eq.), DCC (0.45 g, 2,19 mmol, 1.00 eq.) and DMAP (0.02 g, $0.18 \mathrm{mmol}, 0.08$ eq.) were added in this order. The reaction mixture was stirred overnight at room temperature. Afterwards, sat. $\mathrm{NaHCO}_{3}$ solution $(100 \mathrm{~mL})$ was added and the aqueous layer was extracted with EtOAc $(2 \times 100 \mathrm{~mL})$. The combined organic layers were washed with water $(100 \mathrm{~mL})$ and saturated aqueous $\mathrm{NaCl}$ solution $(100 \mathrm{~mL})$, dried over $\mathrm{MgSO}_{4}$ and the solvent was removed under reduced pressure. Purification by flash chromatography on silica gel ( $n$-pentane/acetone, $5 / 1$ ) yielded the target compound $(\mathbf{2 6}, 1.10 \mathrm{~g}, 1.69 \mathrm{mmol}, 77 \%)$ as a mixture of four isomers and as yellow solid.

TLC: $R_{\mathrm{f}}=0.57(n$-pentane/acetone, 5/1).

${ }^{1} \mathbf{H}-\mathbf{N M R}\left(500 \mathrm{MHz}, \mathrm{CDCl}_{3}\right): \delta(\mathrm{ppm})=1.12-1.17\left(\mathrm{~m}, 1 \mathrm{H}, 1^{\prime}-\mathrm{CH}_{3}\right.$ (erythro)), $1.21-1.27$ $\left(\mathrm{m}, 2 \mathrm{H}, 1^{\prime}-\mathrm{CH}_{3}\right.$ (threo)), $1.28-1.35\left(\mathrm{~m}, 3 \mathrm{H}, 3^{\prime}-\mathrm{CH}_{3}\right), 1.42,1.44,1.45,1.47(4 \times \mathrm{s}, 9 \mathrm{H}$, $\mathrm{O} t \mathrm{Bu}), 2.60-3.05\left(\mathrm{~m}, 2 \mathrm{H}, \beta-\mathrm{H}_{2}\right), 3.72-3.97$ (m, $7 \mathrm{H}, 3^{\prime}-\mathrm{H}, 4^{\prime \prime}-\mathrm{OMe}, 5$ "-OMe), $4.18-4.56$ (m, $4 \mathrm{H}, \alpha-\mathrm{H}$, Fmoc-9-H, Fmoc-10- $\left.\mathrm{H}_{2}\right), 5.11-5.25\left(\mathrm{~m}, 1 \mathrm{H}, 2^{\prime}-\mathrm{H}\right), 5.55-5.78(\mathrm{~m}, 1 \mathrm{H}, \mathrm{NH})$, $6.74-6.90(\mathrm{~m}, 1 \mathrm{H}, 6 "-\mathrm{H}), 7.30(\mathrm{t}, \mathrm{J}=7.4 \mathrm{~Hz}, 2 \mathrm{H}$, Fmoc-2-H, Fmoc-7-H), 7.36 - 7.43 (m, 3 H, 3"-H, Fmoc-3-H, Fmoc-6-H), $7.54-7.62$ (m, 2 H, Fmoc-1-H, Fmoc-8-H), 7.76 (d, $J=7.4 \mathrm{~Hz}, 2 \mathrm{H}$, Fmoc-4-H, Fmoc-5-H).

${ }^{13} \mathrm{C}-\mathrm{NMR}\left(125 \mathrm{MHz}, \mathrm{CDCl}_{3}\right): \delta(\mathrm{ppm})=16.65\left(\mathrm{C}-3^{\prime}\right), 18.12\left(\mathrm{C}-1^{\prime}\right), 27.84\left(\mathrm{OC}\left(\mathrm{CH}_{3}\right)_{3}\right)$, 36.87 (C- $\beta$ ), 38.08 (C-3'), 47.06 (Fmoc-C-9), 50.89 (C- $\alpha$ ), 56.15 (OMe), 56.21 (OMe), 67.20 (Fmoc-C-10), 74.48 (C-2'), $82.64\left(\mathrm{OC}\left(\mathrm{CH}_{3}\right)_{3}\right), 107.68$ (C-3"), 109.69 (C-6"), 119.95 (Fmoc-C-4, Fmoc-C-5), 125.09, 125.14 (Fmoc-C-1, Fmoc-C-8), 127.03 (Fmoc-C-2, Fmoc-C-7), 127.68 (Fmoc-C-3, Fmoc-C-6), 131.83 (C-1"), 141.23 (Fmoc-C-4a, Fmoc-C-4b), 142.89 (C-2"), 143.77, 143.84 (Fmoc-C-8a, Fmoc-C-9a), 147.21 (C-4"), 152.51 (C-5"), 155.85 (Fmoc-CO), 
$169.37\left(\mathrm{CO}_{2} t \mathrm{Bu}\right), 170.24(\delta-\mathrm{CO})$.

ESI-MS(+) $(\mathrm{m} / z(\%)): 649.3(10)[\mathrm{M}+\mathrm{H}]^{+}, 671.3(100)[\mathrm{M}+\mathrm{Na}]^{+}, 687.2(5)[\mathrm{M}+\mathrm{K}]^{+}$, $1319.5(59)[2 \mathrm{M}+\mathrm{Na}]^{+}$.

HR-MS (ESI):

calculated for $\left[\mathrm{C}_{35} \mathrm{H}_{40} \mathrm{~N}_{2} \mathrm{O}_{10} \mathrm{Na}\right]^{+}\left([\mathrm{M}+\mathrm{Na}]^{+}\right): 671.2575$; found: 671.2569 . 


\section{Synthesis of $N$-Fmoc- $\delta$-[3-(4,5-dimethoxy-2-nitrophenyl)-2-butyl]-L-aspartic acid (Fmoc- Asp(DMNPB)-OH (27) $)^{[135]}$}

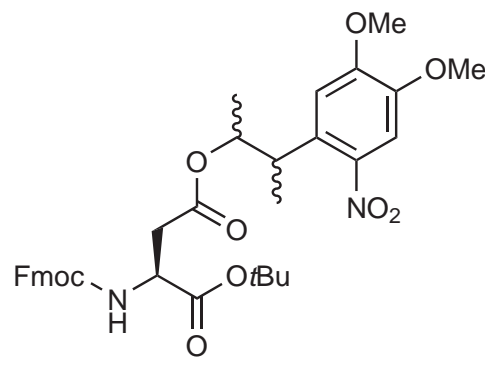

26

Fmoc-Asp(DMNPB)-OtBu

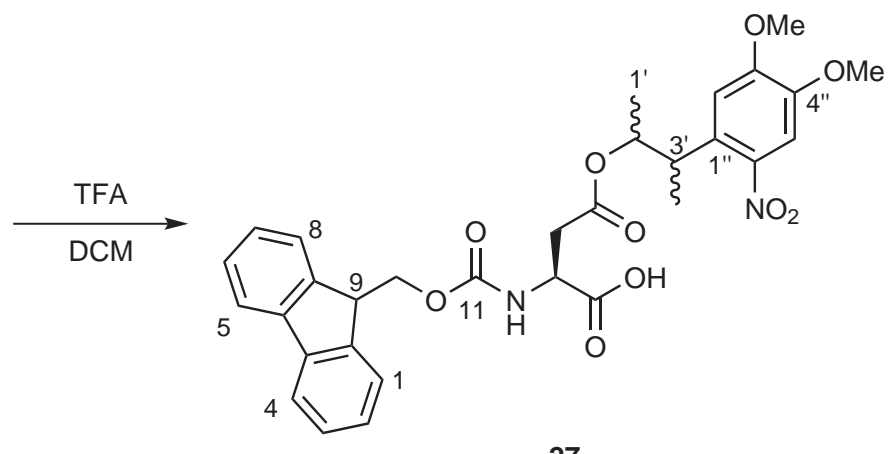

27

Fmoc-Asp(DMNPB)-OH

$\mathrm{C}_{31} \mathrm{H}_{32} \mathrm{~N}_{2} \mathrm{O}_{10}$ $592.2 \mathrm{~g} / \mathrm{mol}$

Fmoc-Asp(DMNPB)-OtBu (26, $1.04 \mathrm{~g}, 1.61 \mathrm{mmol}, 1.00 \mathrm{eq}$.$) was dissolved in a mixture of$ TFA/DCM $(1 / 1,(v / v), 100 \mathrm{~mL})$ and the solution was stirred at room temperature for $2 \mathrm{~h}$. The solvent was removed by nitrogen stream and the residue was coevaporated by $\mathrm{Et}_{2} \mathrm{O}(3 \mathrm{x})$ and toluene $(3 \mathrm{x})$ under reduced pressure. Purification by flash chromatography on silica gel (DCM/MeOH/AcOH, 20/1/0.05 $(v / v / v))$ followed by preperative RP-HPLC $(65 \%-100 \%$, $30 \mathrm{~min})$ and lyophilisation yielded the target compound (27, $0.70 \mathrm{~g}, 1.18 \mathrm{mmol}, 73 \%$ ) as a light-yellow solid.

TLC: $R_{\mathrm{f}}=0.45(\mathrm{DCM} / \mathrm{MeOH}, 20 / 1(v / v))$.

HPLC (Macherey-Nagel NUCLEODUR ${ }^{\circledR}$ RP-C18 ec analytical column (100 $\left., 5 \mu \mathrm{m}, 250 \times 4.6 \mathrm{~mm}\right)$, gradient: $50-100 \% \mathrm{~B}$ in $30 \mathrm{~min}$ ): $t_{\mathrm{R} \text {, netto }}=16.77 \mathrm{~min}, t_{\mathrm{R} \text {, brutto }}=19.79 \mathrm{~min}$.

${ }^{1} \mathbf{H}-\mathbf{N M R}\left(300 \mathrm{MHz}, \mathrm{CDCl}_{3}\right): \delta(\mathrm{ppm})=1.16\left(\mathrm{~d}, J=6.2 \mathrm{~Hz}, 1 \mathrm{H}, 1\right.$ '- $\mathrm{CH}_{3}$ (erythro)), $1.23-1.35\left(\mathrm{~m}, 5 \mathrm{H}, 1^{\prime}-\mathrm{CH}_{3}\right.$ (threo), 3'- $\left.\mathrm{CH}_{3}\right), 2.65-2.98\left(\mathrm{~m}, 2 \mathrm{H}, \beta-\mathrm{H}_{2}\right), 3.75-3.96(\mathrm{~m}$, $7 \mathrm{H}, 3$ '-H, 4"-OMe, 5"-OMe), $4.17-4.49$ (m, 3 H, Fmoc-9-H, Fmoc-10-H $)_{2}, 4.54-4.63(\mathrm{~m}$, $1 \mathrm{H}, \alpha-\mathrm{H}), 5.21\left(\mathrm{dq}, J=6.2,2.2 \mathrm{~Hz}, 1 \mathrm{H}, 2^{\prime}-\mathrm{H}\right), 5.72(\mathrm{t}, J=8.7 \mathrm{~Hz}, 1 \mathrm{H}, \mathrm{NH}), 6.84(\mathrm{~d}$, $J=3.3 \mathrm{~Hz}, 1 \mathrm{H}, 6 "-\mathrm{H}), 7.30$ (t, J=7.3 Hz, $2 \mathrm{H}$, Fmoc-2-H, Fmoc-7-H), $7.35(\mathrm{~s}, 1 \mathrm{H}, 3$ "-H), 7.39 (t, J = 7.3 Hz, 3 H, Fmoc-3-H, Fmoc-6-H, 3"-H), 7.58 (d, J=7.3 Hz, 2 H, Fmoc-1-H, Fmoc-8-H), 7.75 (d, J = 7.3 Hz, $2 \mathrm{H}$, Fmoc-4-H, Fmoc-5-H).

${ }^{13} \mathrm{C}-\mathrm{NMR}\left(125 \mathrm{MHz}, \mathrm{CDCl}_{3}\right): \delta(\mathrm{ppm})=16.38$ (C-3'), 17.99 (C-1'), $36.50(\mathrm{C}-\beta), 38.42$ (C-3'), 47.03 (Fmoc-C-9), 50.17 (C- $\alpha$ ), 56.18 (OMe), 56.25 (OMe), 67.40 (Fmoc-C-10), 74.95 (C-2'), 107.72 (C-3"), 109.60 (C-6"), 119.98 (Fmoc-C-4, Fmoc-C-5), 125.10, 125.15 (Fmoc-C- 
1, Fmoc-C-8), 127.07 (Fmoc-C-2, Fmoc-C-7), 127.73 (Fmoc-C-3, Fmoc-C-6), 131.85 (C-1"), 141.26 (Fmoc-C-4a, Fmoc-C-4b), 142.81 (C-2"), 143.65, 143.71 (Fmoc-C-8a, Fmoc-C-9a), 147.27 (C-4"), 152.57 (C-5"), 156.00 (Fmoc-CO), $170.16(\delta-\mathrm{CO}), 174.40\left(\mathrm{CO}_{2} \mathrm{H}\right)$.

ESI-MS(+) $(m / z(\%)): 593.2(7)[\mathrm{M}+\mathrm{H}]^{+}, 615.2(100)[\mathrm{M}+\mathrm{Na}]^{+}, 631.2(7)[\mathrm{M}+\mathrm{K}]^{+}$, $1207.5(41)[2 \mathrm{M}+\mathrm{Na}]^{+}$.

ESI-MS(-) $(m / z(\%)): 591.2(100)[M-H]^{-}, 705.2(5)[M+T F A-H]^{-}$.

HR-MS (ESI):

calculated for $\left[\mathrm{C}_{31} \mathrm{H}_{31} \mathrm{~N}_{2} \mathrm{O}_{10}\right]^{-}\left([\mathrm{M}-\mathrm{H}]^{-}\right)$: 591.1984; found: 591.1985,

calculated for $\left[\mathrm{C}_{31} \mathrm{H}_{32} \mathrm{~N}_{2} \mathrm{O}_{10} \mathrm{Na}\right]^{+}\left([\mathrm{M}+\mathrm{Na}]^{+}\right)$: 615.1949; found: 615.1950. 


\subsection{Caged Synaptobrevin-2 analogues}

\subsubsection{Synthesis of Test Peptides 28 - 33}

Synthesis of Synaptobrevin-2 (54 - 60) R56R(Nvoc) (28)<smiles>COc1cc(COC(=O)NC(=N)NCCC[C@H](NC(=O)[C@H](CCC(=O)O)NC(=O)[C@H](N)CC(C)C)C(=O)N[C@@H](CC(=O)O)C(=O)N[C@@H](CCC(N)=O)C(=O)N[C@@H](CCCCN)C(=O)N[C@@H](CC(C)C)C(=O)O)c([N+](=O)[O-])cc1OC</smiles>

Peptide 28 was synthesized according to GSP 1 (chapter 8.4.1) by manual solid phase peptide synthesis $(25 \mu \mathrm{mol})$ on a preloaded Fmoc-Leu-WANG resin $(0.32 \mathrm{mmol} / \mathrm{g})$. The following amino acid derivatives were used:

Fmoc-Arg(Nvoc)-OH (21), Fmoc-Asp(OtBu)-OH, Fmoc-Gln(Trt)-OH, Fmoc-Glu(OtBu)-OH, Fmoc-Leu-OH, Fmoc-Lys(Boc)-OH.

Single coupling was performed with a coupling time of $30 \mathrm{~min}(45 \mathrm{~min}$ for 21 ) and successful coupling was checked by performing a KAISER test (chapter 8.4.4).

After complete synthesis peptide 28 was cleaved from the resin $(\sim 120 \mathrm{mg})$ and completely deprotected according to GSP 3 (chapter 8.4.3). After purification by HPLC peptide 28 could not be obtained probably due to instability of caged amino acid building block under SPPS conditions. A compound was isolated with a difference of mass to charge ratio of $\Delta \frac{m}{z}=-50$ according to ESI-MS but could not be assigned to a truncated sequence or possible side products. 


\section{Synthesis of Synaptobrevin-2 (54 - 60) K59K(Nvoc) (29)}<smiles>COc1cc(COC(=O)NCCCC[C@H](NC(=O)[C@H](CCC(N)=O)NC(=O)[C@H](CC(=O)O)NC(=O)[C@H](CCCNC(=N)N)NC(=O)[C@H](CCC(=O)O)NC(=O)[C@H](N)CC(C)C)C(=O)N[C@@H](CC(C)C)C(=O)O)c([N+](=O)[O-])cc1OC</smiles>

29

Peptide 29 was synthesized according to GSP 1 (chapter 8.4.1) by manual solid phase peptide synthesis $(0.05 \mathrm{mmol})$ on a preloaded Fmoc-Leu-WANG resin $(0.32 \mathrm{mmol} / \mathrm{g})$. The following amino acid derivatives were used:

Fmoc-Arg(Pbf)-OH, Fmoc-Asp(OtBu)-OH, Fmoc-Gln(Trt)-OH, Fmoc-Glu(OtBu)-OH, FmocLeu-OH, Fmoc-Lys(Nvoc)-OH (16).

Single coupling was performed with a coupling time of $30 \mathrm{~min}$ (45 min for 16) and successful coupling was checked by performing a KAISER test (chapter 8.4.4).

After complete synthesis peptide 29 was cleaved from half of the resin ( $120 \mathrm{mg})$ and completely deprotected according to GSP 3 (chapter 8.4.3). The rest of the resin-bound peptide ( $\sim 120 \mathrm{mg}$ ) was used for synthesis of peptide $\mathbf{3 0}$. After purification by HPLC peptide $\mathbf{2 9}$ (14.4 mg, $12.6 \mu \mathrm{mol}, 51 \%$ ) was obtained as light-yellow solid.

HPLC (Macherey-Nagel NUCLEODUR ${ }^{\circledR}$ RP-C18 ec analytical column (100 A, $\left.5 \mu \mathrm{m}, 250 \times 4.6 \mathrm{~mm}\right)$, gradient: $10-60 \% \mathrm{~B}$ in $30 \mathrm{~min}$ ): $t_{\mathrm{R}, \text { netto }}=22.56 \mathrm{~min}, t_{\mathrm{R}, \text { brutto }}=26.59 \mathrm{~min}$.

UV-vis $\left(\mathrm{H}_{2} \mathrm{O} / \mathrm{MeCN}(9 / 1), 500 \mu \mathrm{M}\right): \lambda_{\max }(\mathrm{nm})=204,242,300,347$.

ESI-MS(+) $(m / z(\%)): 570.8(100)[\mathrm{M}+2 \mathrm{H}]^{2+}, 1140.5(7)[\mathrm{M}+\mathrm{H}]^{+}$.

\section{HR-MS (ESI):}

calculated for $\left[\mathrm{C}_{48} \mathrm{H}_{79} \mathrm{~N}_{13} \mathrm{O}_{19}\right]^{2+}\left([\mathrm{M}+2 \mathrm{H}]^{2+}\right)$ : 570.7802 ; found: 570.7807 , calculated for $\left[\mathrm{C}_{48} \mathrm{H}_{78} \mathrm{~N}_{13} \mathrm{O}_{19}\right]^{+}\left([\mathrm{M}+\mathrm{H}]^{+}\right)$: 1140.5531 ; found: 1140.5526 . 


\section{Synthesis of Synaptobrevin-2 (51 - 60) K52K(Nvoc), K59K(Nvoc) (30)}<smiles>COc1cc(COC(=O)NCCCC[C@H](NC(=O)[C@H](CCC(N)=O)NC(=O)[C@H](CC(=O)O)NC(=O)[C@H](CCCNC(=N)N)NC(=O)[C@H](CCC(=O)O)NC(=O)[C@H](CC(C)C)NC(=O)[C@H](NC(=O)[C@H](CCCCNC(=O)OCc2cc(OC)c(OC)cc2[N+](=O)[O-])NC(=O)[C@H](N)CC(=O)O)C(C)C)C(=O)N[C@@H](CC(C)C)C(=O)O)c([N+](=O)[O-])cc1OC</smiles>

Peptide 30 was synthesized according to GSP 1 (chapter 8.4.1) by manual solid phase peptide synthesis $(25 \mu \mathrm{mol})$ on the preloaded WANG resin $(\sim 120 \mathrm{mg})$ obtained from the synthesis of peptide 29. The following amino acid derivatives were used:

Fmoc-Asp(OtBu)-OH, Fmoc-Lys(Nvoc)-OH (16), Fmoc-Val-OH.

Single coupling was performed with a coupling time of $30 \mathrm{~min}(45 \mathrm{~min}$ for 16) and successful coupling was checked by performing a KAISER test (chapter 8.4.4).

After the complete synthesis, peptide $\mathbf{3 0}$ was cleaved from the resin and completely deprotected according to GSP 3 (chapter 8.4.3). After purification by HPLC peptide 30 (20.3 mg, $11.77 \mu \mathrm{mol}, 47 \%$ ) was obtained as light-yellow solid.

HPLC (Macherey-Nagel NUCLEODUR ${ }^{\circledR}$ RP-C18 ec analytical column (100 A, $\left.5 \mu \mathrm{m}, 250 \times 4.6 \mathrm{~mm}\right)$, gradient: $30-75 \% \mathrm{~B}$ in $30 \mathrm{~min}$ ): $t_{\mathrm{R}, \text { netto }}=17.36 \mathrm{~min}, t_{\mathrm{R} \text {, brutto }}=20.40 \mathrm{~min}$.

UV-vis $\left(\mathrm{H}_{2} \mathrm{O} / \mathrm{MeCN}(1 / 1), 500 \mu \mathrm{M}\right): \lambda_{\max }(\mathrm{nm})=210,242,303,353$.

ESI-MS(+) (m/z (\%)): $861.4(64)[\mathrm{M}+2 \mathrm{H}]^{2+}, 1720.8(100)[\mathrm{M}]$ (deconvoluted).

\section{HR-MS (ESI):}

calculated for $\left[\mathrm{C}_{73} \mathrm{H}_{114} \mathrm{~N}_{18} \mathrm{O}_{30}\right]^{2+}\left([\mathrm{M}+2 \mathrm{H}]^{2+}\right)$ : 861.3969; found: 861.3970, calculated for $\left[\mathrm{C}_{73} \mathrm{H}_{112} \mathrm{~N}_{18} \mathrm{O}_{30}\right][\mathrm{M}]:$ 1720.8; found: 1720.8 (deconvoluted). 


\section{Synthesis of Synaptobrevin-2 (54 - 60) D57D(DMNPB) (31)}

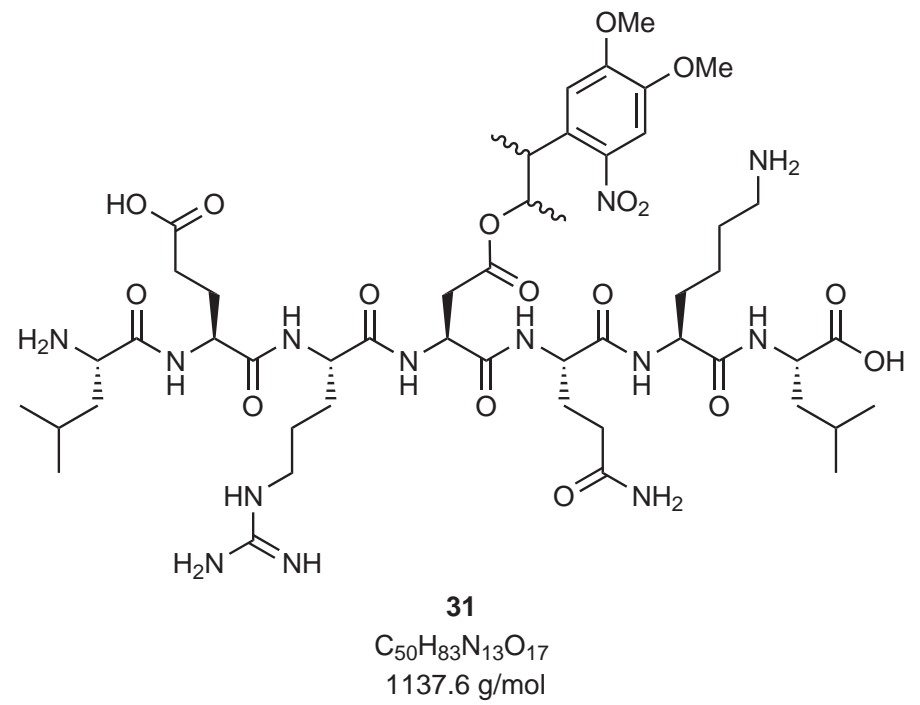

Peptide 31 was synthesized according to GSP 1 (chapter 8.4.1) by manual solid phase peptide synthesis $(25 \mu \mathrm{mol})$ on preloaded Fmoc-Leu-WANG resin $(0.32 \mathrm{mmol} / \mathrm{g})$. The following amino acid derivatives were used:

Fmoc-Arg(Pbf)-OH, Fmoc-Asp(DMNPB)-OH (27), Fmoc-Gln(Trt)-OH, Fmoc-Glu(OtBu)-OH, Fmoc-Leu-OH, Fmoc-Lys(Boc)-OH.

Single coupling was performed with a coupling time of $30 \mathrm{~min}$ (45 min for 27 ) and successful coupling was checked by performing a KAISER test (chapter 8.4.4).

After the complete synthesis, peptide $\mathbf{3 1}$ was cleaved from the resin and completely deprotected according to GSP 3 (chapter 8.4.3). After purification by HPLC, peptide 31 (22.6 mg, $19.9 \mu \mathrm{mol}, 79 \%$ ) was obtained as a light-yellow solid.

HPLC (Macherey-Nagel NUCLEODUR ${ }^{\circledR}$ RP-C18 ec analytical column (100 A, $\left.5 \mu \mathrm{m}, 250 \times 4.6 \mathrm{~mm}\right)$, gradient: $10-60 \% \mathrm{~B}$ in $30 \mathrm{~min}$ ): $t_{\mathrm{R} \text {,netto }}=21.05 \mathrm{~min}, t_{\mathrm{R}, \text { brutto }}=24.97 \mathrm{~min}$.

UV-vis $\left(\mathrm{H}_{2} \mathrm{O} / \mathrm{MeCN}(9 / 1), 500 \mu \mathrm{M}\right): \lambda_{\max }(\mathrm{nm})=199,246,304,348$.

ESI-MS(+) $(m / z(\%)): 380.2(100)[M+3 H]^{3+}, 569.8(43)[\mathrm{M}+2 \mathrm{H}]^{2+}, 1138.6(1)[\mathrm{M}+\mathrm{H}]^{+}$.

\section{HR-MS (ESI):}

calculated for $\left[\mathrm{C}_{50} \mathrm{H}_{86} \mathrm{~N}_{13} \mathrm{O}_{17}\right]^{3+}\left([\mathrm{M}+3 \mathrm{H}]^{3+}\right)$ : 380.2083; found: 380.2087 , calculated for $\left[\mathrm{C}_{50} \mathrm{H}_{85} \mathrm{~N}_{13} \mathrm{O}_{17}\right]^{2+}\left([\mathrm{M}+2 \mathrm{H}]^{2+}\right)$ : 569.8088; found: 569.8085 , calculated for $\left[\mathrm{C}_{50} \mathrm{H}_{84} \mathrm{~N}_{13} \mathrm{O}_{17}\right]^{+}\left([\mathrm{M}+\mathrm{H}]^{+}\right)$: 1138.6103; found: 1138.6105 . 


\section{Synthesis of Synaptobrevin-2 (54 - 60) (32)}<smiles>CC(C)C[C@H](N)C(=O)N[C@@H](CCC(=O)O)C(=O)N[C@@H](CCCNC(=N)N)C(=O)N[C@@H](CC(=O)O)C(=O)N[C@@H](CCC(N)=O)C(=O)N[C@@H](CCCCN)C(=O)N[C@@H](CC(C)C)C(=O)O</smiles>

32

$\mathrm{C}_{38} \mathrm{H}_{68} \mathrm{~N}_{12} \mathrm{O}_{13}$

$900.5 \mathrm{~g} / \mathrm{mol}$

Peptide 32 was synthesized according to GSP 1 (chapter 8.4.1) by manual solid phase peptide synthesis $(50 \mu \mathrm{mol})$ on preloaded Fmoc-Leu-WANG resin $(0.32 \mathrm{mmol} / \mathrm{g})$. The following amino acid derivatives were used:

Fmoc-Arg(Pbf)-OH, Fmoc-Asp(OtBu)-OH, Fmoc-Gln(Trt)-OH, Fmoc-Glu(OtBu)-OH, FmocLeu-OH, Fmoc-Lys(Boc)-OH.

Single coupling was performed with a coupling time of $30 \mathrm{~min}$ and successful coupling was checked by performing a KAISER test (chapter 8.4.4).

After the complete synthesis peptide 32 was cleaved from half of the resin $(\sim 120 \mathrm{mg})$ and completely deprotected according to GSP 3 (chapter 8.4.3). The rest of the resin-bound peptide ( $\sim 120 \mathrm{mg}$ ) was used for synthesis of peptide 33. After purification by HPLC peptide 32 was obtained as colorless solid.

HPLC (Macherey-Nagel NUCLEODUR ${ }^{\circledR}$ RP-C18 ec analytical column (100 $\left., 5 \mu \mathrm{m}, 250 \times 4.6 \mathrm{~mm}\right)$, gradient: $10-60 \% \mathrm{~B}$ in $30 \mathrm{~min}$ ): $t_{\mathrm{R}, \text { netto }}=10.17 \mathrm{~min}, t_{\mathrm{R} \text {, brutto }}=14.04 \mathrm{~min}$.

ESI-MS(+) $(\mathrm{m} / \mathrm{z}(\%)): 301.2(38)[\mathrm{M}+3 \mathrm{H}]^{3+}, 451.3(100)[\mathrm{M}+2 \mathrm{H}]^{2+}, 901.5(14)[\mathrm{M}+\mathrm{H}]^{+}$.

\section{HR-MS (ESI):}

calculated for $\left[\mathrm{C}_{38} \mathrm{H}_{71} \mathrm{~N}_{12} \mathrm{O}_{13}\right]^{3+}\left([\mathrm{M}+3 \mathrm{H}]^{3+}\right)$ : 301.1749; found: 301.1755 ,

calculated for $\left[\mathrm{C}_{38} \mathrm{H}_{70} \mathrm{~N}_{12} \mathrm{O}_{13}\right]^{2+}\left([\mathrm{M}+2 \mathrm{H}]^{2+}\right)$ : 451.2587; found: 451.2588,

calculated for $\left[\mathrm{C}_{38} \mathrm{H}_{69} \mathrm{~N}_{12} \mathrm{O}_{13}\right]^{+}\left([\mathrm{M}+\mathrm{H}]^{+}\right)$: 901.5102; found: 901.5100 . 


\section{Synthesis of Synaptobrevin-2 (51 - 60) (33)}

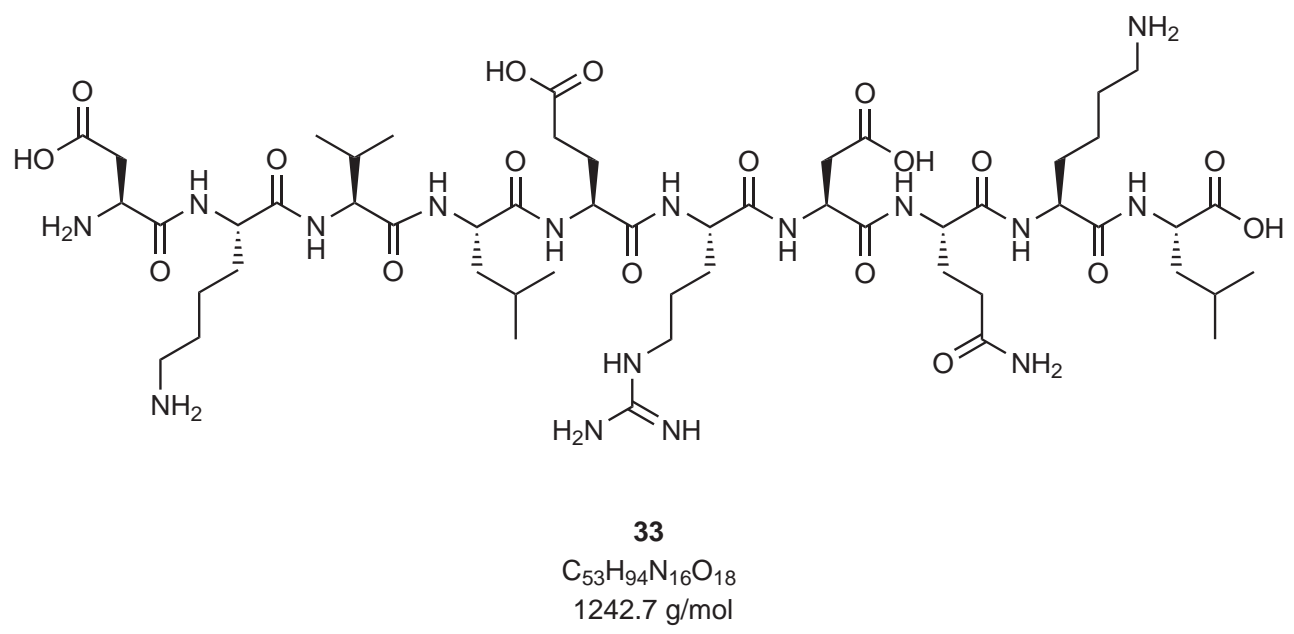

Peptide 33 was synthesized according to GSP 1 (chapter 8.4.1) by manual solid phase peptide synthesis $(25 \mu \mathrm{mol})$ on the preloaded WANG resin $(\sim 120 \mathrm{mg})$ obtained from the synthesis of peptide 32. The following amino acid derivatives were used:

Fmoc-Asp(OtBu)-OH, Fmoc-Lys(Boc)-OH, Fmoc-Val-OH.

Single coupling was performed with a coupling time of $30 \mathrm{~min}$ and successful coupling was checked by performing a KAISER test (chapter 8.4.4).

After the complete synthesis, peptide 33 was cleaved from the resin and completely deprotected according to GSP 3 (chapter 8.4.3). After purification by HPLC peptide 33 was obtained as colorless solid.

HPLC (Macherey-Nagel NUCLEODUR ${ }^{\circledR}$ RP-C18 ec analytical column (100 Å, $\left.5 \mu \mathrm{m}, 250 \times 4.6 \mathrm{~mm}\right)$, gradient: $10-75 \% \mathrm{~B}$ in $30 \mathrm{~min}$ ): $t_{\mathrm{R}, \text { netto }}=11.20 \mathrm{~min}, t_{\mathrm{R}, \text { brutto }}=15.17 \mathrm{~min}$.

ESI-MS(+) $(m / z(\%)): 415.2(100)[\mathrm{M}+3 \mathrm{H}]^{3+}, 622.3(49)[\mathrm{M}+2 \mathrm{H}]^{2+}, 1243.7(1)[\mathrm{M}+\mathrm{H}]^{+}$.

HR-MS (ESI):

calculated for $\left[\mathrm{C}_{53} \mathrm{H}_{97} \mathrm{~N}_{16} \mathrm{O}_{18}\right]^{3+}\left([\mathrm{M}+3 \mathrm{H}]^{3+}\right)$ : 415.2383; found: 415.2388, calculated for $\left[\mathrm{C}_{53} \mathrm{H}_{96} \mathrm{~N}_{16} \mathrm{O}_{18}\right]^{2+}\left([\mathrm{M}+2 \mathrm{H}]^{2+}\right)$ : 622.3539; found: 622.3531, calculated for $\left[\mathrm{C}_{53} \mathrm{H}_{95} \mathrm{~N}_{16} \mathrm{O}_{18}\right]^{+}\left([\mathrm{M}+\mathrm{H}]^{+}\right)$: 1243.7005; found: 1243.6985 . 


\subsubsection{Synthesis of Synaptobrevin-2 (61 - 84) (34)}

H-SELDDRADALQAGASQFETSAAKL $-O H$

34

$\mathrm{C}_{104} \mathrm{H}_{168} \mathrm{~N}_{30} \mathrm{O}_{41}$

$2493.2 \mathrm{~g} / \mathrm{mol}$

Peptide 34 was synthesized according to GSP 2 (chapter 8.4.2) by automated solid phase peptide synthesis $(0.10 \mathrm{mmol})$ on a preloaded Fmoc-Leu-WANG resin $(0.32 \mathrm{mmol} / \mathrm{g})$ using the CEM LibertyBlue peptide synthesizer. The following amino acid derivatives were used:

Fmoc-Ala-OH, Fmoc-Arg(Pbf)-OH, Fmoc-Asp(OtBu)-OH, Fmoc-Gln(Trt)-OH, Fmoc-Glu(OtBu)$\mathrm{OH}$, Fmoc-Gly-OH, Fmoc-Leu-OH, Fmoc-Lys(Boc)-OH, Fmoc-Phe-OH, Fmoc-Ser( $t \mathrm{Bu})-\mathrm{OH}$, Fmoc-Thr( $t \mathrm{Bu})-\mathrm{OH}$.

According to GSP 3 (chapter 8.4.3) a small amount of peptide 34 was cleaved from the resin and completely deprotected for analysis by HPLC and mass spectrometry. The residual amount was used resin-bound for further syntheses (peptides 35 and 36).

HPLC (Macherey-Nagel NUCLEODUR ${ }^{\circledR}$ RP-C18 ec analytical column $(100 \AA, 5 \mu \mathrm{m}, 250 \times 4.6 \mathrm{~mm})$, gradient: $10-90 \% \mathrm{~B}$ in $30 \mathrm{~min}$ ): $t_{\mathrm{R}, \text { netto }}=13.65 \mathrm{~min}, t_{\mathrm{R}, \text { brutto }}=16.82 \mathrm{~min}$.

ESI-MS(+) $(\mathrm{m} / \mathrm{z}(\%)): 624.6(7)[\mathrm{M}+4 \mathrm{H}]^{4+}, 832.4(100)[\mathrm{M}+3 \mathrm{H}]^{3+}, 1248.1(24)[\mathrm{M}+2 \mathrm{H}]^{2+}$, 2493.2 (100) [M] (deconvoluted).

HR-MS (ESI):

calculated for $\left[\mathrm{C}_{104} \mathrm{H}_{172} \mathrm{~N}_{30} \mathrm{O}_{41}\right]^{4+}\left([\mathrm{M}+4 \mathrm{H}]^{4+}\right)$ : 624.3069; found: 624.3066, calculated for $\left[\mathrm{C}_{104} \mathrm{H}_{171} \mathrm{~N}_{30} \mathrm{O}_{41}\right]^{3+}\left([\mathrm{M}+3 \mathrm{H}]^{3+}\right)$ : 832.0734; found: 832.0742, calculated for $\left[\mathrm{C}_{104} \mathrm{H}_{170} \mathrm{~N}_{30} \mathrm{O}_{41}\right]^{2+}\left([\mathrm{M}+2 \mathrm{H}]^{2+}\right)$ : 1247.6064; found: 1247.6071 , calculated for $\left[\mathrm{C}_{104} \mathrm{H}_{168} \mathrm{~N}_{30} \mathrm{O}_{41}\right]$ ([M]): 2493.2; found: 2493.2 (deconvoluted). 


\title{
8.8.3 Synthesis of Synaptobrevin-2 (51 - 84) K52K(Nvoc), D57D(DMNPB), K59K(Nvoc) (35)
}

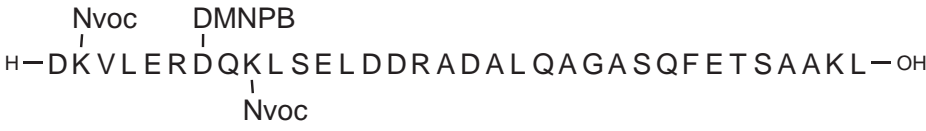 \\ 35 \\ $\mathrm{C}_{189} \mathrm{H}_{293} \mathrm{~N}_{49} \mathrm{O}_{74}$ \\ $4433.1 \mathrm{~g} / \mathrm{mol}$
}

Peptide 35 was synthesized according to GSP 1 (chapter 8.4.1) by manual solid phase peptide synthesis $(100 \mu \mathrm{mol})$ on the preloaded WANG resin $(\sim 544 \mathrm{mg})$ obtained from the synthesis of peptide 34. The following amino acid derivatives were used:

Fmoc-Arg(Pbf)-OH, Fmoc-Asp(DMNPB)-OH (27), Fmoc-Asp(OtBu)-OH, Fmoc-Gln(Trt)$\mathrm{OH}$, Fmoc-Glu(OtBu)-OH, Fmoc-Leu-OH, Fmoc-Lys(Boc)-OH, Fmoc-Lys(Nvoc)-OH (16), Fmoc-Val-OH.

Double coupling was performed with a coupling time of $2 \times 30 \mathrm{~min}(2 \times 45 \mathrm{~min}$ for $\mathbf{1 6}$ and 27) and successful coupling was checked by performing a KAISER test (chapter 8.4.4).

According to GSP 3 (chapter 8.4.3) a small amount of peptide 35 was cleaved from the resin and completely deprotected for analysis by HPLC and mass spectrometry. The residual amount was used resin-bound for further synthesis (peptide 36 ).

HPLC (Macherey-Nagel NUCLEODUR ${ }^{\circledR}$ RP-C18 ec analytical column (100 Å, $\left.5 \mu \mathrm{m}, 250 \times 4.6 \mathrm{~mm}\right)$, gradient: $10-90 \% \mathrm{~B}$ in $30 \mathrm{~min}$ ): $t_{\mathrm{R} \text {,netto }}=21.66 \mathrm{~min}, t_{\mathrm{R}, \text { brutto }}=24.97 \mathrm{~min}$.

ESI-MS(+) $(m / z(\%)): 888.0(9)[\mathrm{M}+5 \mathrm{H}]^{5+}, 1109.8(100)[\mathrm{M}+4 \mathrm{H}]^{4+}, 1479.3(19)[\mathrm{M}+3 \mathrm{H}]^{3+}$, 4433.1 (100) [M] (deconvoluted).

\section{HR-MS (ESI):}

calculated for $\left[\mathrm{C}_{189} \mathrm{H}_{298} \mathrm{~N}_{49} \mathrm{O}_{74}\right]^{5+}\left([\mathrm{M}+5 \mathrm{H}]^{5+}\right)$ : 887.6207; found: 887.6210, calculated for $\left[\mathrm{C}_{189} \mathrm{H}_{297} \mathrm{~N}_{49} \mathrm{O}_{74}\right]^{4+}\left([\mathrm{M}+4 \mathrm{H}]^{4+}\right)$ : 1109.2740 ; found: 1109.2754 , calculated for $\left[\mathrm{C}_{189} \mathrm{H}_{296} \mathrm{~N}_{49} \mathrm{O}_{74}\right]^{3+}\left([\mathrm{M}+3 \mathrm{H}]^{3+}\right)$ : 1478.6963 ; found: 1478.6975 , calculated for $\left[\mathrm{C}_{189} \mathrm{H}_{293} \mathrm{~N}_{49} \mathrm{O}_{74}\right]$ ([M]): 4433.1; found: 4433.1 (deconvoluted). 


\subsubsection{Synthesis of Synaptobrevin-2 (28 - 84) K52K(Nvoc), D57D(DMNPB), K59K(Nvoc) (36)}

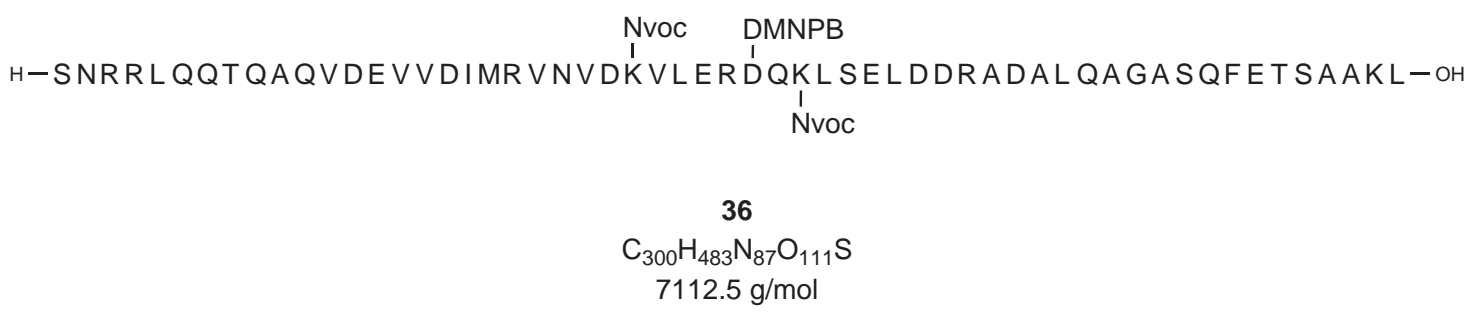

Peptide 36 was synthesized according to GSP 2 (chapter 8.4.2) by automated solid phase peptide synthesis $(0.10 \mathrm{mmol})$ on the preloaded WANG resin obtained from the synthesis of peptide 35 using the CEM LibertyBlue peptide synthesizer. Standard coupling methods were applied expect for R30, R31 (0.10-Tripple Arg Coupling (HS)). The following amino acid derivatives were used:

Fmoc-Ala-OH, Fmoc-Arg(Pbf)-OH, Fmoc-Asn(Trt)-OH, Fmoc-Asp(OtBu)-OH, Fmoc-Gln(Trt)$\mathrm{OH}$, Fmoc-Glu(OtBu)-OH, Fmoc-lle-OH, Fmoc-Leu-OH, Fmoc-Met-OH, Fmoc-Ser $(t \mathrm{Bu})-\mathrm{OH}$, Fmoc-Thr(tBu)-OH, Fmoc-Val-OH.

After the complete synthesis, peptide $\mathbf{3 6}$ was cleaved from the resin and completely deprotected according to GSP 3 (chapter 8.4.3). After purification by HPLC peptide 36 (5.50 mg, $7.73 \mu \mathrm{mol}, 1.6 \%$ ) was obtained only in insufficient purity as a colorless solid.

HPLC (Macherey-Nagel NUCLEODUR ${ }^{\circledR}$ RP-C18 ec semipreperative column (100, $5 \mu \mathrm{m}$, $250 \times 10.0 \mathrm{~mm}$ ), gradient: $20-70 \% \mathrm{~B}$ in $30 \mathrm{~min}): t_{\mathrm{R}, \text { netto }}=16.45 \mathrm{~min}, t_{\mathrm{R}, \text { brutto }}=21.55 \mathrm{~min}$.

ESI-MS(+) $(m / z(\%)): 1017.5(25)[\mathrm{M}+7 \mathrm{H}]^{7+}, 1187.1(100)[\mathrm{M}+6 \mathrm{H}]^{6+}, 1424.3(45)$ $[\mathrm{M}+5 \mathrm{H}]^{5+}, 1779.9(13)[\mathrm{M}+4 \mathrm{H}]^{4+}, 7112.4(100)[\mathrm{M}]$ (deconvoluted).

HR-MS (ESI):

calculated for $\left[\mathrm{C}_{300} \mathrm{H}_{490} \mathrm{~N}_{87} \mathrm{O}_{111} \mathrm{~S}\right]^{7+}\left([\mathrm{M}+7 \mathrm{H}]^{7+}\right)$ : 1017.5020; found: 1017.5006, calculated for $\left[\mathrm{C}_{300} \mathrm{H}_{489} \mathrm{~N}_{87} \mathrm{O}_{111} \mathrm{~S}\right]^{6+}\left([\mathrm{M}+6 \mathrm{H}]^{6+}\right)$ : 1187.0848; found: 1187.0858, calculated for $\left[\mathrm{C}_{300} \mathrm{H}_{488} \mathrm{~N}_{87} \mathrm{O}_{111} \mathrm{~S}\right]^{5+}\left([\mathrm{M}+5 \mathrm{H}]^{5+}\right): 1424.3003$; found: 1424.3017 , calculated for $\left[\mathrm{C}_{300} \mathrm{H}_{487} \mathrm{~N}_{87} \mathrm{O}_{111} \mathrm{~S}\right]^{4+}\left([\mathrm{M}+4 \mathrm{H}]^{4+}\right)$ : 1779.8730; found: 1779.8716 , calculated for $\left[\mathrm{C}_{300} \mathrm{H}_{483} \mathrm{~N}_{87} \mathrm{O}_{111} \mathrm{~S}\right]$ ([M]): 7112.5 ; found: 7112.4 (deconvoluted). 


\title{
8.9 Labelled SNARE transmembrane domains ${ }^{[139-142, ~ 144] ~}$
}

\subsubsection{Synthesis of Syntaxin-4 (262 - 297) Homo sapiens (37)}

\author{
H-ALENQKKARKKKVLI AI CVSI TVVLLAVI'I GVTVVG-OH \\ 37 \\ $\mathrm{C}_{174} \mathrm{H}_{316} \mathrm{~N}_{46} \mathrm{O}_{44} \mathrm{~S}$ \\ $3788.4 \mathrm{~g} / \mathrm{mol}$
}

Peptide 37 was synthesised according to GSP 2 (chapter 8.4.2) by using the CEM Liberty12 peptide synthesizer. For the synthesis the batch size of $0.10 \mathrm{mmol}$ with douple coupling procedure (0.10-Double-Capping) was used as well as the Fmoc-Gly-WANG resin LL $(0.3 \mathrm{mmol} / \mathrm{g}$, $333 \mathrm{mg}$ ). For the amino acids Cys (0.10-Double-Capping 50C) and Arg (0.10-Double-Capping $\operatorname{Arg}(K M))$ special programs were used. After completion of the automated synthesis the resin was transferred from Falcon Tube in a $10 \mathrm{~mL}-B D$-syringe, washed with DCM $(15 \times 8 \mathrm{~mL})$ and dried under reduced pressure. A small amount of the peptide $\mathbf{3 7}$ was cleaved from the resin and completely deprotected according to GSP 3 (chapter 8.4.3) by using $250 \mu \mathrm{L}$ of cleavage mixture containing EDT. The rest of the resin was used for labelling reactions (peptides 41 and $\mathbf{4 5}$ ).

ESI-MS(+) $(m / z(\%)): 758.68(29)[\mathrm{M}+5 \mathrm{H}]^{5+}, 948.10(31)[\mathrm{M}+4 \mathrm{H}]^{4+}, 3788.37(60)$ $[\mathrm{M}+\mathrm{H}]^{+}$.

HR-MS (ESI):

calculated for $\left[\mathrm{C}_{174} \mathrm{H}_{321} \mathrm{~N}_{46} \mathrm{O}_{44} \mathrm{~S}\right]^{5+}\left([\mathrm{M}+5 \mathrm{H}]^{5+}\right)$ : 758.6809; found: 758.6812,

calculated for $\left[\mathrm{C}_{174} \mathrm{H}_{320} \mathrm{~N}_{46} \mathrm{O}_{44} \mathrm{~S}\right]^{4+}\left([\mathrm{M}+4 \mathrm{H}]^{4+}\right)$ : 948.0993; found: 948.0987,

calculated for $\left[\mathrm{C}_{174} \mathrm{H}_{317} \mathrm{~N}_{46} \mathrm{O}_{44} \mathrm{~S}\right]^{+}\left([\mathrm{M}+\mathrm{H}]^{+}\right)$: 3788.37; found: 3788.37 . 


\subsubsection{Synthesis of Syntaxin-1A (254 - 288) Homo sapiens (38)}

H-AVKYQSKARRKKI MI I I CCVI LGI VI ASTVGGI FA-OH

$$
\begin{gathered}
38 \\
\mathrm{C}_{172} \mathrm{H}_{300} \mathrm{~N}_{46} \mathrm{O}_{41} \mathrm{~S}_{3} \\
3762.2 \mathrm{~g} / \mathrm{mol}
\end{gathered}
$$

Peptide 38 was synthesised according to GSP 2 (chapter 8.4.2) by using the CEM Liberty12 peptide synthesizer. For the synthesis the batch size of $0.10 \mathrm{mmol}$ with douple coupling procedure (0.10-Double-Capping) was used as well as the Fmoc-Ala-WANG resin LL $(0.38 \mathrm{mmol} / \mathrm{g}$, $263 \mathrm{mg}$ ). For the amino acids Cys (0.10-Double-Capping 50C) and Arg (0.10-Double-Capping $\operatorname{Arg}(K M))$ special programs were used. After completion of the automated synthesis the resin was transferred from Falcon Tube in a $10 \mathrm{~mL}-B D$-syringe, washed with DCM $(15 \times 8 \mathrm{~mL})$ and dried under reduced pressure. A small amount of the peptide $\mathbf{3 8}$ was cleaved from the resin and completely deprotected according to GSP 3 (chapter 8.4 .3 ) by using $250 \mu \mathrm{L}$ of cleavage mixture containing EDT. The rest of the resin was used for labelling reactions (peptide 42, 46 and 48).

ESI-MS(+) $(\mathrm{m} / \mathrm{z}(\%)): 628.4(12)[\mathrm{M}+6 \mathrm{H}]^{6+}, 753.9(100)[\mathrm{M}+5 \mathrm{H}]^{5+}, 942.1(87)[\mathrm{M}+4 \mathrm{H}]^{4+}$, $1255.7(8)[\mathrm{M}+3 \mathrm{H}]^{3+}, 3762.2(100)[\mathrm{M}]$ (deconvoluted).

\section{HR-MS (ESI):}

calculated for $\left[\mathrm{C}_{172} \mathrm{H}_{306} \mathrm{~N}_{46} \mathrm{O}_{41} \mathrm{~S}_{3}\right]^{6+}\left([\mathrm{M}+6 \mathrm{H}]^{6+}\right)$ : 628.0400; found: 628.0392, calculated for $\left[\mathrm{C}_{172} \mathrm{H}_{305} \mathrm{~N}_{46} \mathrm{O}_{41} \mathrm{~S}_{3}\right]^{5+}\left([\mathrm{M}+5 \mathrm{H}]^{5+}\right)$ : 753.4466; found: 753.4467 , calculated for $\left[\mathrm{C}_{172} \mathrm{H}_{304} \mathrm{~N}_{46} \mathrm{O}_{41} \mathrm{~S}_{3}\right]^{5+}\left([\mathrm{M}+4 \mathrm{H}]^{4+}\right)$ : 941.5564 ; found: 941.5568 , calculated for $\left[\mathrm{C}_{172} \mathrm{H}_{303} \mathrm{~N}_{46} \mathrm{O}_{41} \mathrm{~S}_{3}\right]^{5+}\left([\mathrm{M}+3 \mathrm{H}]^{3+}\right)$ : 1255.0728; found: 1255.0727 , calculated for $\left[\mathrm{C}_{172} \mathrm{H}_{300} \mathrm{~N}_{46} \mathrm{O}_{41} \mathrm{~S}_{3}\right][\mathrm{M}]: 3762.2$; found: 3762.2 (deconvoluted). 


\subsubsection{Synthesis of Syntaxin-1A (254 - 288) K264A, K265A Homo sapiens (39)}

H-AVKYQSKARRAAI MIII CCVI LGI VI ASTVGGI FA-OH

39

$\mathrm{C}_{166} \mathrm{H}_{286} \mathrm{~N}_{44} \mathrm{O}_{41} \mathrm{~S}_{3}$

$3648.0 \mathrm{~g} / \mathrm{mol}$

Peptide 39 was synthesised according to GSP 2 (chapter 8.4 .2 ) by using the CEM Liberty12 peptide synthesizer. For the synthesis the batch size of $0.10 \mathrm{mmol}$ with douple coupling procedure (0.10-Double-Capping) was used as well as the Fmoc-Ala-WANG resin LL $(0.38 \mathrm{mmol} / \mathrm{g}$, $263 \mathrm{mg}$ ). For the amino acids Cys (0.10-Double-Capping 50C) and Arg (0.10-Double-Capping $\operatorname{Arg}(K M))$ special programs were used. After completion of the automated synthesis the resin was transferred from Falcon Tube in a $10 \mathrm{~mL}-B D$-syringe, washed with DCM $(15 \times 8 \mathrm{~mL})$ and dried under reduced pressure. A small amount of the peptide $\mathbf{3 9}$ was cleaved from the resin and completely deprotected according to GSP 3 (chapter 8.4.3) by using $250 \mu \mathrm{L}$ of cleavage mixture containing EDT. The rest of the resin was used for labelling reactions (peptide 43).

\section{HR-MS (ESI):}

calculated for $\left[\mathrm{C}_{166} \mathrm{H}_{290} \mathrm{~N}_{44} \mathrm{O}_{41} \mathrm{~S}_{3}\right]^{4+}\left([\mathrm{M}+4 \mathrm{H}]^{4+}\right)$ : 913.03; found: 913.02, calculated for $\left[\mathrm{C}_{166} \mathrm{H}_{289} \mathrm{~N}_{44} \mathrm{O}_{41} \mathrm{~S}_{3}\right]^{3+}\left([\mathrm{M}+3 \mathrm{H}]^{3+}\right)$ : 1217.03 ; found: 1217.03 , calculated for [ $\mathrm{C}_{166} \mathrm{H}_{286} \mathrm{~N}_{44} \mathrm{O}_{41} \mathrm{~S}_{3}$ ] [M]: 3648.1; found: 3648.1 (deconvoluted). 


\title{
8.9.4 Synthesis of Syntaxin-1A (257 - 288) M267A, C271A, I279A Rattus norvegicus (40)[142]
}

\author{
H-YQSKARRKK I A I I I ACV I LG I I AAST I GG I FG-OH \\ 40 \\ $\mathrm{C}_{154} \mathrm{H}_{266} \mathrm{~N}_{42} \mathrm{O}_{38} \mathrm{~S}$ \\ $3344.0 \mathrm{~g} / \mathrm{mol}$
}

Peptide $\mathbf{4 0}$ was synthesised according to GSP 2 (chapter 8.4.2) by using the CEM Liberty12 peptide synthesizer. For the synthesis the batch size of $0.10 \mathrm{mmol}$ with douple coupling procedure (0.10-Double-Capping) was used as well as the Fmoc-Gly-WANG resin LL $(0.3 \mathrm{mmol} / \mathrm{g}$, $333 \mathrm{mg}$ ). For the amino acids Cys (0.10-Double-Capping 50C) and Arg (0.10-Double-Capping $\operatorname{Arg}(K M))$ special programs were used. After completion of the automated synthesis the resin was transferred from Falcon Tube in a $10 \mathrm{~mL}-B D$-syringe, washed with DCM $(15 \times 8 \mathrm{~mL})$ and dried under reduced pressure. A small amount of the peptide $\mathbf{4 0}$ was cleaved from the resin and completely deprotected according to GSP 3 (chapter 8.4.3) by using $250 \mu \mathrm{L}$ of cleavage mixture containing EDT. The rest of the resin was used for labelling reactions (peptide 44, 47 and 49).

ESI-MS(+) $(\mathrm{m} / \mathrm{z}(\%)): 670.0(33)[\mathrm{M}+5 \mathrm{H}]^{5+}, 837.5(100)[\mathrm{M}+4 \mathrm{H}]^{4+}, 1116.0(19)[\mathrm{M}+3 \mathrm{H}]^{3+}$, $1673.5(8)[\mathrm{M}+2 \mathrm{H}]^{2+}, 3343.9(100)[\mathrm{M}]$ (deconvoluted).

HR-MS (ESI):

calculated for $\left[\mathrm{C}_{154} \mathrm{H}_{271} \mathrm{~N}_{42} \mathrm{O}_{38} \mathrm{~S}\right]^{5+}\left([\mathrm{M}+5 \mathrm{H}]^{5+}\right)$ : 669.8052; found: 669.8051, calculated for $\left[\mathrm{C}_{154} \mathrm{H}_{270} \mathrm{~N}_{42} \mathrm{O}_{38} \mathrm{~S}\right]^{4+}\left([\mathrm{M}+4 \mathrm{H}]^{4+}\right)$ : 837.0046; found: 837.0046, calculated for $\left[\mathrm{C}_{154} \mathrm{H}_{269} \mathrm{~N}_{42} \mathrm{O}_{38} \mathrm{~S}\right]^{3+}\left([\mathrm{M}+3 \mathrm{H}]^{3+}\right)$ : 1116.0047 ; found: 1116.0062 , calculated for $\left[\mathrm{C}_{154} \mathrm{H}_{268} \mathrm{~N}_{42} \mathrm{O}_{38} \mathrm{~S}\right]^{2+}\left([\mathrm{M}+2 \mathrm{H}]^{2+}\right)$ : 1673.5034 ; found: 1673.5022 , calculated for [ $\mathrm{C}_{154} \mathrm{H}_{266} \mathrm{~N}_{42} \mathrm{O}_{38} \mathrm{~S}$ ] [M]: 3344.0; found: 3344.0 (deconvoluted). 


\subsubsection{Synthesis of Atto647N-labelled Syntaxin-4 262 - 297 Homo sapiens $(41)^{[139,}$ 141]}

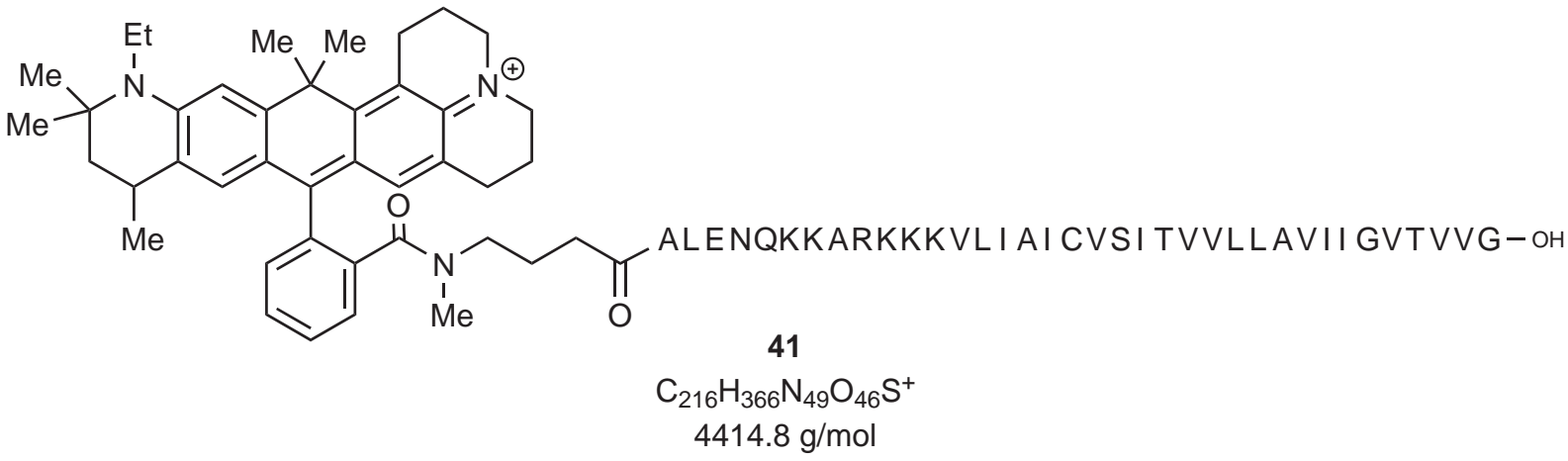

The resin-bound peptide 37 (chapter 8.9.1) was swollen in NMP $(2.00 \mathrm{~mL}$ ) for $1 \mathrm{~h}$ and to remove the final Fmoc-protecting group piperidine $(20 \%)$ in NMP $(2 \times 1.00 \mathrm{~mL})$ was added and shaken for $15 \mathrm{~min}$. The resin was washed with NMP $(5 \times 2.00 \mathrm{~mL}), \mathrm{DCM}(5 \times 2.00 \mathrm{~mL})$ and NMP $(5 \times 2.00 \mathrm{~mL})$. Afterwards, a solution of Atto647N-NHS-ester $(1.00 \mathrm{mg}, 1.19 \mu \mathrm{mol}$, 1.00 eq.), PyBOP (1.23 mg, $2.37 \mu \mathrm{mol}, 2.00$ eq.) and DIPEA (10.0 $\mu \mathrm{L}, 51.8 \mu \mathrm{mol}, 44.0$ eq.) in NMP $(150 \mu \mathrm{L})$ was added to the resin-bound peptide $37(11.0 \mathrm{mg}, 2.37 \mu \mathrm{mol}, 2.00 \mathrm{eq}$.) and shaken overnight under light exclusion. The solution was filtrated and the resin was washed with NMP $(5 \times 2.00 \mathrm{~mL})$ and DCM $(5 \times 2.00 \mathrm{~mL})$ and dried under reduced pressure. The peptide $\mathbf{4 1}$ was cleaved from the resin and completely deprotected according to GSP 3 (chapter 8.4.3) by using EDT containing cleavage mixture $(500 \mu \mathrm{L})$. After precipitation and drying under reduced pressure, the crude peptide was suspended in $\mathrm{H}_{2} \mathrm{O} / \mathrm{MeCN}$ and lyophilised to obtain peptide $\mathbf{4 1}$ as a dark blue solid.

ESI-MS(+) $(m / z(\%)): 883.95(18)[\mathrm{M}+5 \mathrm{H}]^{5+}, 1104.69(32)[\mathrm{M}+4 \mathrm{H}]^{4+}, 1472.58(25)$ $[\mathrm{M}+3 \mathrm{H}]^{3+}, 4414.75$ (48) [M] (deconvoluted).

\section{HR-MS (ESI):}

calculated for $\left[\mathrm{C}_{216} \mathrm{H}_{371} \mathrm{~N}_{49} \mathrm{O}_{46} \mathrm{~S}\right]^{5+}\left([\mathrm{M}+5 \mathrm{H}]^{5+}\right)$ : 883.9534; found: 883.9538, calculated for $\left[\mathrm{C}_{216} \mathrm{H}_{370} \mathrm{~N}_{49} \mathrm{O}_{46} \mathrm{~S}\right]^{4+}\left([\mathrm{M}+4 \mathrm{H}]^{4+}\right)$ : 1104.6942; found: 1104.6944 , calculated for $\left[\mathrm{C}_{216} \mathrm{H}_{369} \mathrm{~N}_{49} \mathrm{O}_{46} \mathrm{~S}\right]^{3+}\left([\mathrm{M}+3 \mathrm{H}]^{3+}\right)$ : 1472.5821 ; found: 1472.5825 , calculated for $\left[\mathrm{C}_{216} \mathrm{H}_{366} \mathrm{~N}_{49} \mathrm{O}_{46} \mathrm{~S}\right.$ ] [M]: 4414.75; found: 4414.75 (deconvoluted). 


\subsubsection{Synthesis of Atto647N-labelled Syntaxin-1A (254 - 288) Homo sapiens (42)[139-141]}

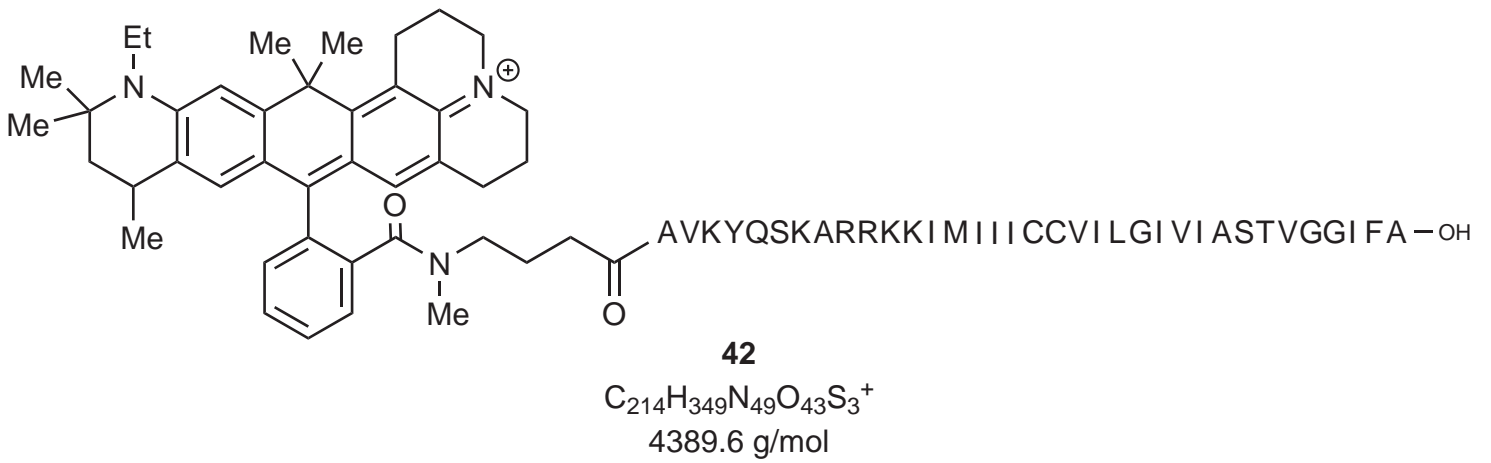

The resin-bound peptide 38 (chapter 8.9.2) was swollen in NMP $(2.00 \mathrm{~mL}$ ) for $1 \mathrm{~h}$ and to remove the final Fmoc-protecting group piperidine $(20 \%)$ in NMP $(2 \times 1.00 \mathrm{~mL})$ was added and shaken for $2 \times 15 \mathrm{~min}$. The resin was washed with NMP $(5 \times 2.00 \mathrm{~mL}), \mathrm{DCM}(5 \times 2.00 \mathrm{~mL})$ and NMP $(5 \times 2.00 \mathrm{~mL})$. Afterwards, a solution of Atto647N-NHS-ester $(1.00 \mathrm{mg}, 1.19 \mu \mathrm{mol}$, 1.00 eq.) and DIPEA $(10.0 \mu \mathrm{L}, 51.8 \mu \mathrm{mol}, 44.0$ eq. $)$ in NMP $(250 \mu \mathrm{L})$ was added to the resin-bound peptide 38 (11.4 $\mathrm{mg}, 2.37 \mu \mathrm{mol}, 2.00$ eq.) and shaken overnight under light exclusion. The solution was filtrated and the resin was washed with NMP $(5 \times 2.00 \mathrm{~mL})$ and DCM $(10 \times 2.00 \mathrm{~mL})$ and dried under reduced pressure. The peptide 42 was cleaved from the resin and completely deprotected according to GSP 3 (chapter 8.4.3) by using EDT containing cleavage mixture $(500 \mu \mathrm{L})$. After precipitation and drying under reduced pressure, the crude peptide was suspended in $\mathrm{H}_{2} \mathrm{O} / \mathrm{MeCN}$ and lyophilised to obtain peptide $\mathbf{4 2}$ as a dark blue solid.

ESI-MS(+) $(\mathrm{m} / z(\%)): 733.1(52)[\mathrm{M}+6 \mathrm{H}]^{6+}, 879.3(100)[\mathrm{M}+5 \mathrm{H}]^{5+}, 1098.9(66)[\mathrm{M}+4 \mathrm{H}]^{4+}$, 4389.6 (100) [M] (deconvoluted).

HR-MS (ESI):

calculated for $\left[\mathrm{C}_{214} \mathrm{H}_{355} \mathrm{~N}_{49} \mathrm{O}_{43} \mathrm{~S}_{3}\right]^{6+}\left([\mathrm{M}+6 \mathrm{H}]^{6+}\right)$ : 732.6038; found: 732.6035,

calculated for $\left[\mathrm{C}_{214} \mathrm{H}_{354} \mathrm{~N}_{49} \mathrm{O}_{43} \mathrm{~S}_{3}\right]^{5+}\left([\mathrm{M}+5 \mathrm{H}]^{5+}\right)$ : 878.9231; found: 878.9219,

calculated for $\left[\mathrm{C}_{214} \mathrm{H}_{353} \mathrm{~N}_{49} \mathrm{O}_{43} \mathrm{~S}_{3}\right]^{4+}\left([\mathrm{M}+4 \mathrm{H}]^{4+}\right)$ : 1098.4021; found: 1098.3996,

calculated for $\left[\mathrm{C}_{214} \mathrm{H}_{349} \mathrm{~N}_{49} \mathrm{O}_{43} \mathrm{~S}_{3}\right]$ [M]: 4389.6; found: 4389.6 (deconvoluted). 


\subsubsection{Synthesis of Atto647N-labelled Syntaxin-1A (254 - 288) K264A, K265A Homo sapiens (43)[140, 141]}

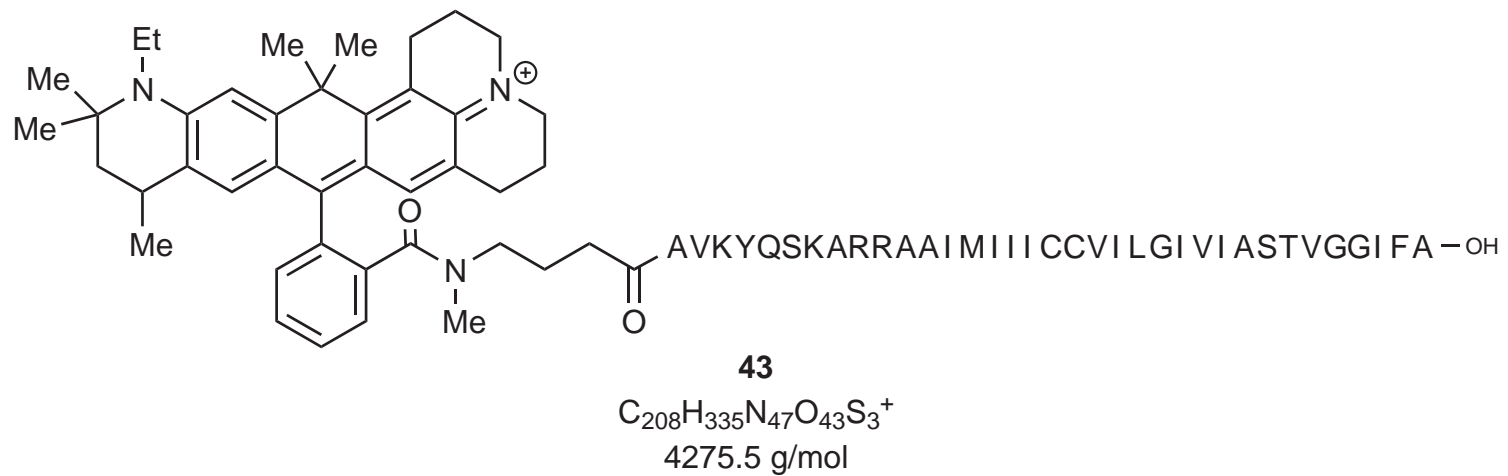

The resin-bound peptide 39 (chapter 8.9.3) was swollen in NMP $(2.00 \mathrm{~mL}$ ) for $1 \mathrm{~h}$ and to remove the final Fmoc-protecting group piperidine $(20 \%)$ in NMP $(2 \times 1.00 \mathrm{~mL})$ was added and shaken for $2 \times 15 \mathrm{~min}$. The resin was washed with NMP $(5 \times 2.00 \mathrm{~mL}), \mathrm{DCM}(5 \times 2.00 \mathrm{~mL})$ and NMP $(5 \times 2.00 \mathrm{~mL})$. Afterwards, a solution of Atto647N-NHS-ester $(1.00 \mathrm{mg}, 1.19 \mu \mathrm{mol}$, 1.00 eq.) and DIPEA $(10.0 \mu \mathrm{L}, 51.8 \mu \mathrm{mol}, 44.0$ eq. $)$ in NMP $(300 \mu \mathrm{L})$ was added to the resin-bound peptide 39 (11.1 mg, $2.37 \mu \mathrm{mol}, 2.00$ eq.) and shaken overnight under light exclusion. The solution was filtrated and the resin was washed with NMP $(5 \times 2.00 \mathrm{~mL})$ and DCM $(10 \times 2.00 \mathrm{~mL})$ and dried under reduced pressure. The peptide 43 was cleaved from the resin and completely deprotected according to GSP 3 (chapter 8.4.3) by using EDT containing cleavage mixture $(500 \mu \mathrm{L})$. After precipitation and drying under reduced pressure, the crude peptide was suspended in $\mathrm{H}_{2} \mathrm{O} / \mathrm{MeCN}$ and lyophilised to obtain peptide 43 as a dark blue solid.

\section{HR-MS (ESI):}

calculated for $\left[\mathrm{C}_{208} \mathrm{H}_{339} \mathrm{~N}_{47} \mathrm{O}_{43} \mathrm{~S}_{3}\right]^{4+}\left([\mathrm{M}+4 \mathrm{H}]^{4+}\right)$ : 1069.87 ; found: 1069.87 , calculated for $\left[\mathrm{C}_{208} \mathrm{H}_{335} \mathrm{~N}_{47} \mathrm{O}_{43} \mathrm{~S}_{3}\right.$ ] [M]: 4275.5; found: 4275.4 (deconvoluted). 


\subsubsection{Synthesis of Atto647N-labelled Syntaxin-1A (257 - 288) M267A, C271A, I279A Rattus norvegicus (44)[139, 141, 142, 144]}

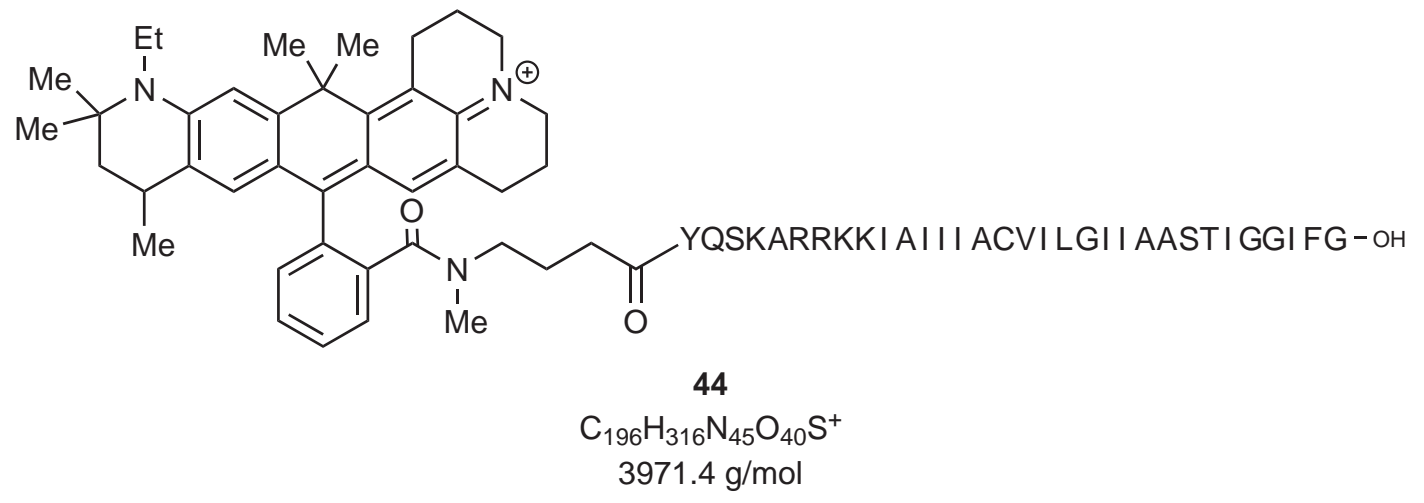

The resin-bound peptide 40 (chapter 8.9.4) was swollen in NMP $(2.00 \mathrm{~mL}$ ) for $1 \mathrm{~h}$ and to remove the final Fmoc-protecting group piperidine $(20 \%)$ in NMP $(2 \times 1.00 \mathrm{~mL})$ was added and shaken for $15 \mathrm{~min}$. The resin was washed with NMP $(5 \times 2.00 \mathrm{~mL}), \mathrm{DCM}(5 \times 2.00 \mathrm{~mL})$ and NMP $(5 \times 2.00 \mathrm{~mL})$. Afterwards, a solution of Atto647N-NHS-ester $(1.00 \mathrm{mg}, 1.19 \mu \mathrm{mol}$, 1.00 eq.), PyBOP (1.23 mg, $2.37 \mu \mathrm{mol}, 2.00$ eq.) and DIPEA (10.0 $\mu \mathrm{L}, 51.8 \mu \mathrm{mol}, 44.0$ eq. $)$ in NMP $(150 \mu \mathrm{L})$ was added to the resin-bound peptide 40 (11.0 mg, $2.37 \mu \mathrm{mol}, 2.00$ eq.) and shaken overnight under light exclusion. The solution was filtrated and the resin was washed with NMP $(5 \times 2.00 \mathrm{~mL})$ and DCM $(5 \times 2.00 \mathrm{~mL})$ and dried under reduced pressure. The peptide $\mathbf{4 4}$ was cleaved from the resin and completely deprotected according to GSP 3 (chapter 8.4.3) by using EDT containing cleavage mixture $(500 \mu \mathrm{L})$. After precipitation and drying under reduced pressure, the crude peptide was suspended in $\mathrm{H}_{2} \mathrm{O} / \mathrm{MeCN}$ and lyophilised to obtain peptide $\mathbf{4 4}$ as a dark blue solid.

ESI-MS(+) $(m / z(\%)): 795.68(21)[\mathrm{M}+5 \mathrm{H}]^{5+}, 994.35(35)[\mathrm{M}+4 \mathrm{H}]^{4+}, 1325.47$ (28) $[\mathrm{M}+3 \mathrm{H}]^{3+}, 3971.37$ (45) $[\mathrm{M}]$ (deconvoluted).

HR-MS (ESI):

calculated for $\left[\mathrm{C}_{196} \mathrm{H}_{321} \mathrm{~N}_{45} \mathrm{O}_{40} \mathrm{~S}\right]^{5+}\left([\mathrm{M}+5 \mathrm{H}]^{5+}\right)$ : 795.6839; found: 795.6841, calculated for $\left[\mathrm{C}_{196} \mathrm{H}_{320} \mathrm{~N}_{45} \mathrm{O}_{40} \mathrm{~S}\right]^{4+}\left([\mathrm{M}+4 \mathrm{H}]^{4+}\right)$ : 994.3530; found: 994.3529, calculated for $\left[\mathrm{C}_{196} \mathrm{H}_{319} \mathrm{~N}_{45} \mathrm{O}_{40} \mathrm{~S}\right]^{3+}\left([\mathrm{M}+3 \mathrm{H}]^{3+}\right)$ : 1325.4683; found: 1325.4693 , calculated for $\left[\mathrm{C}_{196} \mathrm{H}_{316} \mathrm{~N}_{45} \mathrm{O}_{40} \mathrm{~S}\right][\mathrm{M}]$ : 3971.37; found: 3971.37 (deconvoluted). 


\subsubsection{Synthesis of RhodamineRed-labelled Syntaxin-4 (262 - 297) Homo sapiens (45)[139, 141]}

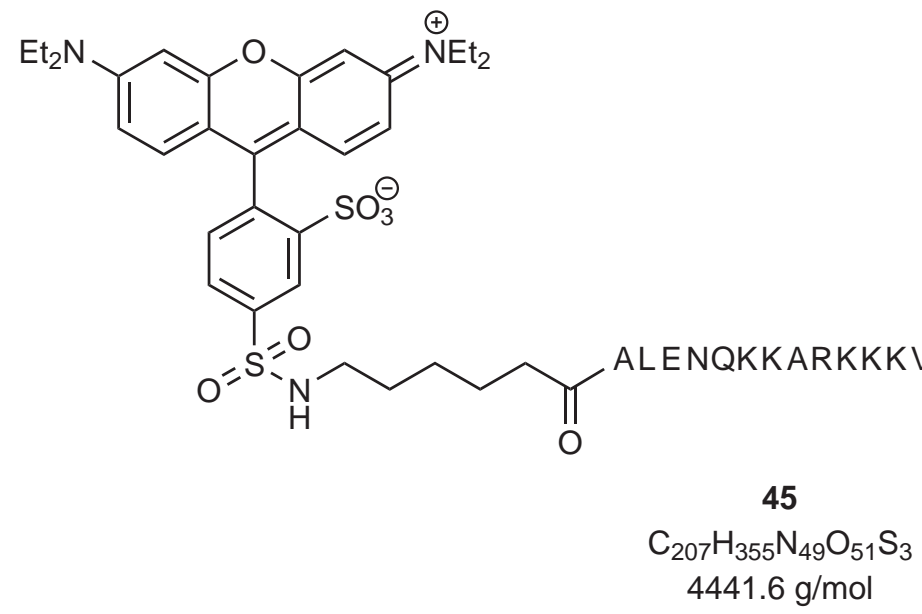

The resin-bound peptide 37 (chapter 8.9.1) was swollen in NMP $(2.00 \mathrm{~mL})$ for $1 \mathrm{~h}$ and to remove the final Fmoc-protecting group piperidine $(20 \%)$ in NMP $(2 \times 1.00 \mathrm{~mL})$ was added and shaken for $15 \mathrm{~min}$. The resin was washed with NMP $(5 \times 2.00 \mathrm{~mL}), \mathrm{DCM}(5 \times 2.00 \mathrm{~mL})$ and NMP $(5 \times 2.00 \mathrm{~mL})$. Afterwards, a solution of RhodamineRed ${ }^{\mathrm{TM}}{ }_{- \text {succinimidylester }}(0.74 \mathrm{mg}$, $0.96 \mu \mathrm{mol}, 1.00$ eq. $)$ and DIPEA $(10.0 \mu \mathrm{L}, 60.0 \mu \mathrm{mol}, 62.5$ eq. $)$ in NMP $(150 \mu \mathrm{L})$ was added to the resin-bound peptide $37(10.0 \mathrm{mg}, 2.00 \mu \mathrm{mol}, 2.08 \mathrm{eq}$.) and shaken overnight under light exclusion. The solution was filtrated and the resin was washed with NMP $(5 \times 2.00 \mathrm{~mL})$ and DCM $(5 \times 2.00 \mathrm{~mL})$ and dried under reduced pressure. The peptide 45 was cleaved from the resin and completely deprotected according to GSP 3 (chapter 8.4.3) by using EDT containing cleavage mixture $(500 \mu \mathrm{L})$. After precipitation and drying under reduced pressure, the crude peptide was suspended in $\mathrm{H}_{2} \mathrm{O} / \mathrm{MeCN}$ and lyophilised to obtain peptide $\mathbf{4 5}$ as a pink solid.

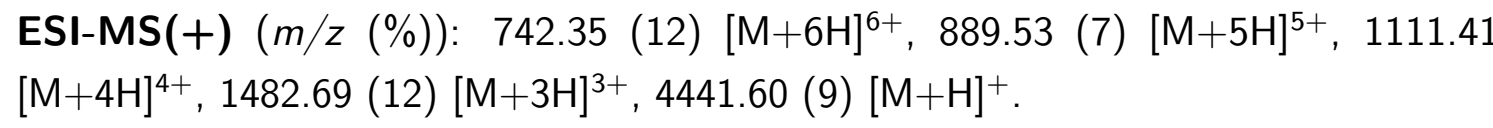

HR-MS (ESI):

calculated for $\left[\mathrm{C}_{207} \mathrm{H}_{356} \mathrm{~N}_{49} \mathrm{O}_{51} \mathrm{~S}_{3}\right]^{+}\left([\mathrm{M}+\mathrm{H}]^{+}\right)$: 4441.59 ; found: 4441.60 . 


\subsubsection{Synthesis of RhodamineRed-labelled Syntaxin-1A (254 - 288) Homo sapiens (46) [139-141]}

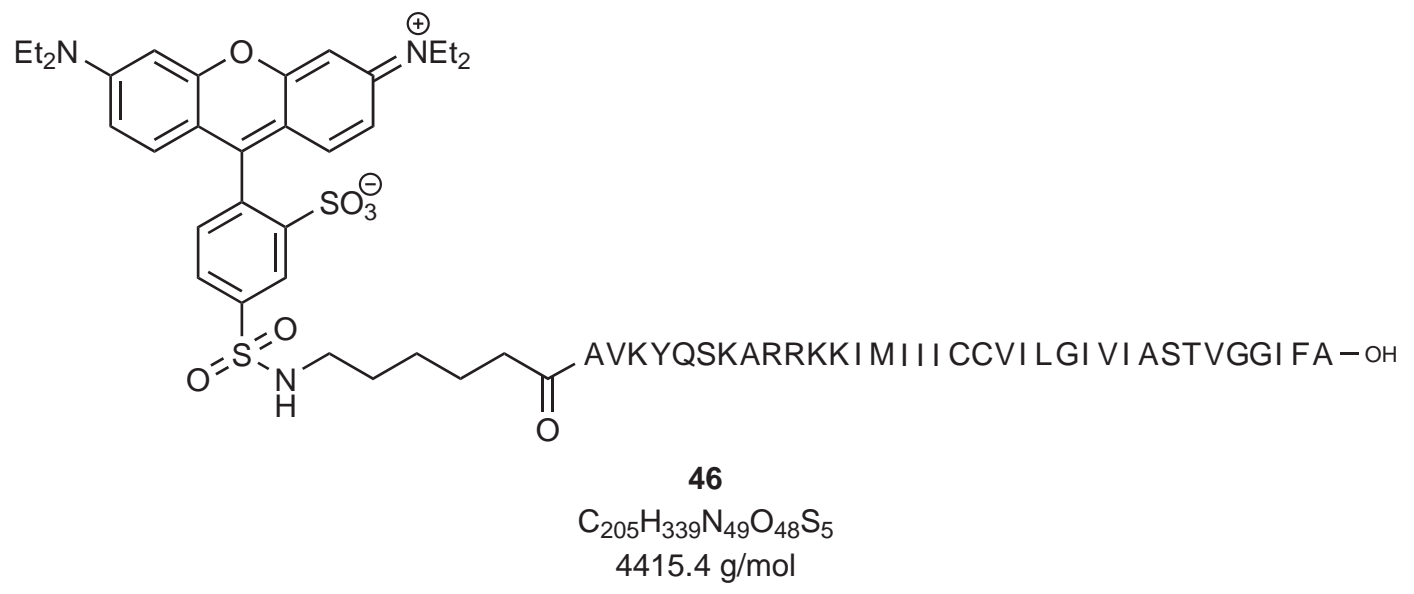

The resin-bound peptide 38 (chapter 8.9.2) was swollen in NMP $(2.00 \mathrm{~mL}$ ) for $1 \mathrm{~h}$ and to remove the final Fmoc-protecting group piperidine $(20 \%)$ in NMP $(2 \times 1.00 \mathrm{~mL})$ was added and shaken for $2 \times 15 \mathrm{~min}$. The resin was washed with NMP $(5 \times 2.00 \mathrm{~mL}), \mathrm{DCM}(5 \times 2.00 \mathrm{~mL})$ and NMP $(5 \times 2.00 \mathrm{~mL})$. Afterwards, a solution of RhodamineRed ${ }^{\mathrm{TM}}$-succinimidylester $(1.00 \mathrm{mg}$, $1.30 \mu \mathrm{mol}, 1.00$ eq.) and DIPEA $(10.0 \mu \mathrm{L}, 60.0 \mu \mathrm{mol}, 46.2$ eq. $)$ in NMP $(250 \mu \mathrm{L})$ was added to the resin-bound peptide 38 (12.5 mg, $2.00 \mu \mathrm{mol}, 2.00$ eq.) and shaken overnight under light exclusion. The solution was filtrated and the resin was washed with NMP $(5 \times 2.00 \mathrm{~mL})$ and DCM $(10 \times 2.00 \mathrm{~mL})$ and dried under reduced pressure. The peptide $\mathbf{4 6}$ was cleaved from the resin and completely deprotected according to GSP 3 (chapter 8.4.3) by using EDT containing cleavage mixture $(500 \mu \mathrm{L})$. After precipitation and drying under reduced pressure, the crude peptide was suspended in $\mathrm{H}_{2} \mathrm{O} / \mathrm{MeCN}$ and lyophilised to obtain peptide $\mathbf{4 6}$ as a pink solid.

ESI-MS(+) $(m / z(\%)): 884.5(100)[\mathrm{M}+5 \mathrm{H}]^{5+}, 1105.6(79)[\mathrm{M}+4 \mathrm{H}]^{4+}, 1473.8(9)[\mathrm{M}+3 \mathrm{H}]^{3+}$, 4415.4 (100) [M] (deconvoluted).

HR-MS (ESI):

calculated for $\left[\mathrm{C}_{205} \mathrm{H}_{345} \mathrm{~N}_{49} \mathrm{O}_{48} \mathrm{~S}_{5}\right]^{6+}\left([\mathrm{M}+6 \mathrm{H}]^{6+}\right)$ : 736.9105; found: 736.9135, calculated for $\left[\mathrm{C}_{205} \mathrm{H}_{344} \mathrm{~N}_{49} \mathrm{O}_{48} \mathrm{~S}_{5}\right]^{5+}\left([\mathrm{M}+5 \mathrm{H}]^{5+}\right)$ : 884.0912; found: 884.0934, calculated for $\left[\mathrm{C}_{205} \mathrm{H}_{343} \mathrm{~N}_{49} \mathrm{O}_{48} \mathrm{~S}_{5}\right]^{4+}\left([\mathrm{M}+4 \mathrm{H}]^{4+}\right)$ : 1104.8622; found: 1104.8644, calculated for $\left[\mathrm{C}_{205} \mathrm{H}_{339} \mathrm{~N}_{49} \mathrm{O}_{48} \mathrm{~S}_{5}\right]$ ([M]): 4415.4; found: 4415.4 (deconvoluted). 


\subsubsection{Synthesis of RhodamineRed-labelled Syntaxin-1A (257 - 288) M267A, C271A, I279A Rattus norvegicus (47)[139, 141]}

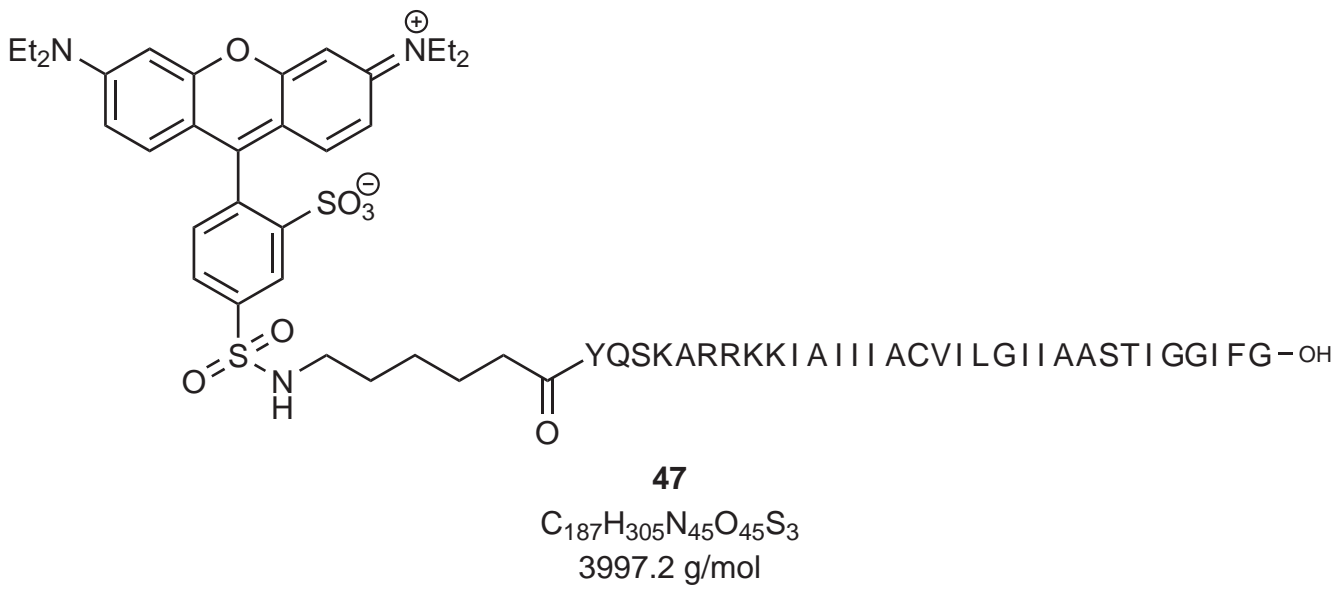

The resin-bound peptide 40 (chapter 8.9.4) was swollen in NMP $(2.00 \mathrm{~mL})$ for $1 \mathrm{~h}$ and to remove the final Fmoc-protecting group piperidine $(20 \%)$ in NMP $(2 \times 1.00 \mathrm{~mL})$ was added and shaken for $2 \times 15 \mathrm{~min}$. The resin was washed with NMP $(5 \times 2.00 \mathrm{~mL}), \mathrm{DCM}(5 \times 2.00 \mathrm{~mL})$ and NMP $(5 \times 2.00 \mathrm{~mL})$. Afterwards, a solution of RhodamineRed ${ }^{\mathrm{TM}}$-succinimidylester $(1.00 \mathrm{mg}$, $1.30 \mu \mathrm{mol}, 1.00$ eq.) and DIPEA $(10.0 \mu \mathrm{L}, 60.0 \mu \mathrm{mol}, 46.2$ eq. $)$ in NMP $(150 \mu \mathrm{L})$ was added to the resin-bound peptide $\mathbf{4 0}(12.0 \mathrm{mg}, 2.60 \mu \mathrm{mol}, 2.00$ eq. $)$ and shaken overnight under light exclusion. The solution was filtrated and the resin was washed with NMP $(5 \times 2.00 \mathrm{~mL})$ and $\operatorname{DCM}(10 \times 2.00 \mathrm{~mL})$ and dried under reduced pressure. The peptide 47 was cleaved from the resin and completely deprotected according to GSP 3 (chapter 8.4.3) by using EDT containing cleavage mixture $(500 \mu \mathrm{L})$. After precipitation and drying under reduced pressure, the crude peptide was suspended in $\mathrm{H}_{2} \mathrm{O} / \mathrm{MeCN}$ and lyophilised to obtain peptide $\mathbf{4 7}$ as a pink solid.

ESI-MS(+) $(m / z(\%)): 1000.3(50)[\mathrm{M}+4 \mathrm{H}]^{4+}, 1333.1(31)[\mathrm{M}+3 \mathrm{H}]^{3+}, 1999.6(2)[\mathrm{M}+2 \mathrm{H}]^{2+}$, 3998.21 (48) [M] (deconvoluted).

HR-MS (ESI):

calculated for $\left[\mathrm{C}_{187} \mathrm{H}_{309} \mathrm{~N}_{45} \mathrm{O}_{45} \mathrm{~S}_{3}\right]^{4+}\left([\mathrm{M}+4 \mathrm{H}]^{4+}\right)$ : 1000.3104 ; found: 1000.3110 , calculated for $\left[\mathrm{C}_{187} \mathrm{H}_{308} \mathrm{~N}_{45} \mathrm{O}_{45} \mathrm{~S}_{3}\right]^{3+}\left([\mathrm{M}+3 \mathrm{H}]^{3+}\right)$ : 1333.4114 ; found: 1333.4124 , calculated for $\left[\mathrm{C}_{187} \mathrm{H}_{307} \mathrm{~N}_{45} \mathrm{O}_{45} \mathrm{~S}_{3}\right]^{2+}\left([\mathrm{M}+2 \mathrm{H}]^{2+}\right)$ : 1999.6134 ; found: 1999.6164 . 


\subsubsection{Synthesis of OregonGreen 488-labelled Syntaxin-1A (254 - 288) Homo sapiens (48)}

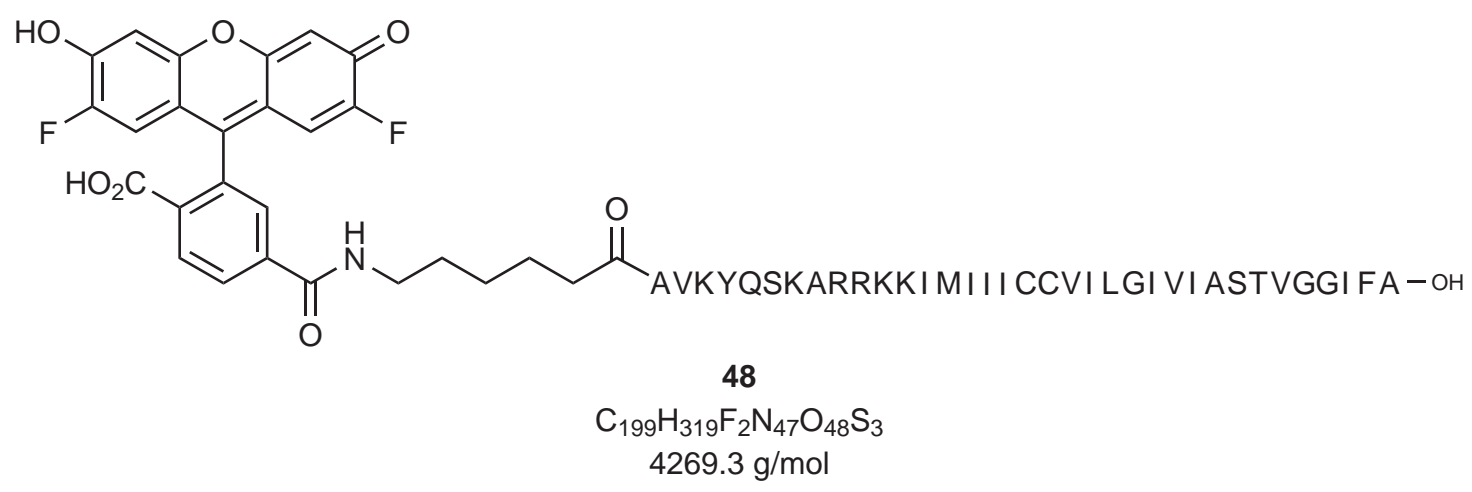

The resin-bound peptide 38 (chapter 8.9.2) was swollen in NMP $(2.00 \mathrm{~mL}$ ) for $1 \mathrm{~h}$ and to remove the final Fmoc-protecting group piperidine $(20 \%)$ in NMP $(2 \times 1.00 \mathrm{~mL})$ was added and shaken for $2 \times 15 \mathrm{~min}$. The resin was washed with NMP $(5 \times 2.00 \mathrm{~mL}), \mathrm{DCM}(5 \times 2.00 \mathrm{~mL})$ and NMP $(5 \times 2.00 \mathrm{~mL})$. Afterwards, a solution of OregonGreen ${ }^{\circledR} 488-X$ succinimidylester $(1.00 \mathrm{mg}$, $1.61 \mu \mathrm{mol}, 1.00$ eq. $)$ and DIPEA $(10.0 \mu \mathrm{L}, 60.0 \mu \mathrm{mol}, 37.3$ eq. $)$ in NMP $(250 \mu \mathrm{L})$ was added to the resin-bound peptide 38 (15.4 mg, $3.21 \mu \mathrm{mol}, 2.00 \mathrm{eq}$.) and shaken overnight under light exclusion. The solution was filtrated and the resin was washed with NMP $(5 \times 2.00 \mathrm{~mL})$ and DCM $(10 \times 2.00 \mathrm{~mL})$ and dried under reduced pressure. The peptide 48 was cleaved from the resin and completely deprotected according to GSP 3 (chapter 8.4.3) by using EDT containing cleavage mixture $(1.00 \mathrm{~mL})$. After precipitation and drying under reduced pressure, the crude peptide was suspended in $\mathrm{H}_{2} \mathrm{O} / \mathrm{MeCN}$ and lyophilised to obtain peptide $\mathbf{4 8}$ as a yellow solid.

ESI-MS(+) $(m / z(\%)): 855.3(83)[\mathrm{M}+5 \mathrm{H}]^{5+}, 1068.9(100)[\mathrm{M}+4 \mathrm{H}]^{4+}, 1424.8(32)$ $[\mathrm{M}+3 \mathrm{H}]^{3+}, 4269.3(100)[\mathrm{M}]$ (deconvoluted).

HR-MS (ESI):

calculated for $\left[\mathrm{C}_{199} \mathrm{H}_{325} \mathrm{~N}_{47} \mathrm{O}_{48} \mathrm{~S}_{3}\right]^{6+}\left([\mathrm{M}+6 \mathrm{H}]^{6+}\right)$ : 712.5589; found: 712.5599, calculated for $\left[\mathrm{C}_{199} \mathrm{H}_{324} \mathrm{~N}_{47} \mathrm{O}_{48} \mathrm{~S}_{3}\right]^{5+}\left([\mathrm{M}+5 \mathrm{H}]^{5+}\right)$ : 854.8692; found: 854.8680, calculated for $\left[\mathrm{C}_{199} \mathrm{H}_{323} \mathrm{~N}_{47} \mathrm{O}_{48} \mathrm{~S}_{3}\right]^{4+}\left([\mathrm{M}+4 \mathrm{H}]^{4+}\right)$ : 1068.3347 ; found: 1068.3323 , calculated for $\left[\mathrm{C}_{199} \mathrm{H}_{322} \mathrm{~N}_{47} \mathrm{O}_{48} \mathrm{~S}_{3}\right]^{3+}\left([\mathrm{M}+3 \mathrm{H}]^{3+}\right)$ : 1424.1105 ; found: 1424.1095, calculated for $\left[\mathrm{C}_{199} \mathrm{H}_{319} \mathrm{~N}_{47} \mathrm{O}_{48} \mathrm{~S}_{3}\right]$ ([M]): 4269.3; found: 4269.3 (deconvoluted). 


\subsubsection{Synthesis of OregonGreen 488-labelled Syntaxin-1A (257 - 288) M267A, C271A, I279A Rattus norvegicus (49)}

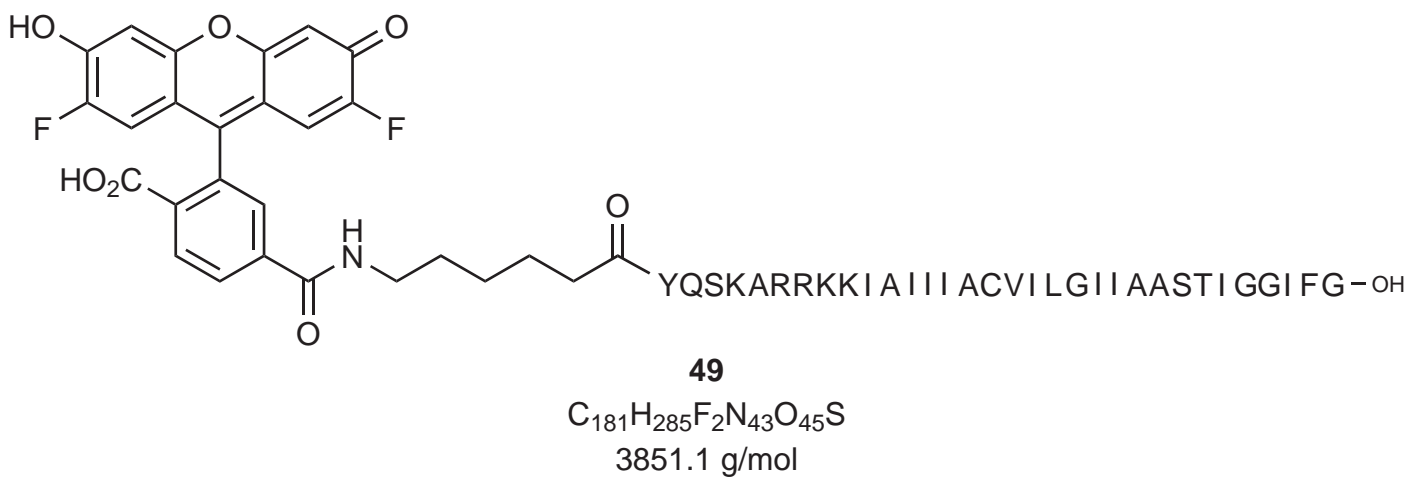

The resin-bound peptide 40 (chapter 8.9.4) was swollen in NMP $(2.00 \mathrm{~mL}$ ) for $1 \mathrm{~h}$ and to remove the final Fmoc-protecting group piperidine $(20 \%)$ in NMP $(2 \times 1.00 \mathrm{~mL})$ was added and shaken for $2 \times 15 \mathrm{~min}$. The resin was washed with NMP $(5 \times 2.00 \mathrm{~mL}), \mathrm{DCM}(5 \times 2.00 \mathrm{~mL})$ and NMP $(5 \times 2.00 \mathrm{~mL})$. Afterwards, a solution of OregonGreen ${ }^{\circledR} 488-X$ succinimidylester $(1.00 \mathrm{mg}$, $1.61 \mu \mathrm{mol}, 1.00$ eq. $)$ and DIPEA $(10.0 \mu \mathrm{L}, 60.0 \mu \mathrm{mol}, 37.3$ eq. $)$ in NMP $(250 \mu \mathrm{L})$ was added to the resin-bound peptide $40(14.6 \mathrm{mg}, 3.21 \mu \mathrm{mol}, 2.00 \mathrm{eq}$.) and shaken overnight under light exclusion. The solution was filtrated and the resin was washed with NMP $(10 \times 2.00 \mathrm{~mL})$ and DCM $(10 \times 2.00 \mathrm{~mL})$ and dried under reduced pressure. The peptide 49 was cleaved from the resin and completely deprotected according to GSP 3 (chapter 8.4.3) by using EDT containing cleavage mixture $(1.00 \mathrm{~mL})$. After precipitation and drying under reduced pressure, the crude peptide was suspended in $\mathrm{H}_{2} \mathrm{O} / \mathrm{MeCN}$ and lyophilised to obtain peptide 49 as a yellow solid.

ESI-MS(+) $(m / z(\%)): 964.3(21)[\mathrm{M}+4 \mathrm{H}]^{4+}, 1285.4(11)[\mathrm{M}+3 \mathrm{H}]^{3+}, 3851.1(27)[\mathrm{M}]$ (deconvoluted).

HR-MS (ESI):

calculated for $\left[\mathrm{C}_{181} \mathrm{H}_{290} \mathrm{~F}_{2} \mathrm{~N}_{43} \mathrm{O}_{45} \mathrm{~S}\right]^{5+}\left([\mathrm{M}+5 \mathrm{H}]^{5+}\right)$ : 771.2277; found: 771.2269, calculated for $\left[\mathrm{C}_{181} \mathrm{H}_{289} \mathrm{~F}_{2} \mathrm{~N}_{43} \mathrm{O}_{45} \mathrm{~S}\right]^{4+}\left([\mathrm{M}+4 \mathrm{H}]^{4+}\right)$ : 963.7829; found: 963.7822 , calculated for $\left[\mathrm{C}_{181} \mathrm{H}_{288} \mathrm{~F}_{2} \mathrm{~N}_{43} \mathrm{O}_{45} \mathrm{~S}\right]^{3+}\left([\mathrm{M}+3 \mathrm{H}]^{3+}\right)$ : 1284.7081 ; found: 1284.7081 , calculated for $\left[\mathrm{C}_{181} \mathrm{H}_{285} \mathrm{~F}_{2} \mathrm{~N}_{43} \mathrm{O}_{45} \mathrm{~S}\right]$ ([M]): 3851.1; found: 3851.1 (deconvoluted). 


\title{
8.9.14 Synthesis of VAMP-3 $(70-100)+C V S$ Rattus norvegicus (50)
}

\author{
H-KLKRKYWWKNCKMWAIGISVLVIIVIIIIVWCVS-OH \\ 50 \\ $\mathrm{C}_{200} \mathrm{H}_{321} \mathrm{~N}_{47} \mathrm{O}_{39} \mathrm{~S}_{3}$ \\ $4101.4 \mathrm{~g} / \mathrm{mol}$
}

Peptide $\mathbf{5 0}$ was synthesised according to GSP 2 (chapter 8.4.2) by using the CEM LibertyBlue peptide synthesizer. For the synthesis the batch size of $0.10 \mathrm{mmol}$ with douple coupling procedure (0.10-Double-Coupling (HS)) was used as well as the Fmoc-Ser(OtBu)-WANG resin $\mathrm{LL}(0.31 \mathrm{mmol} / \mathrm{g}, 323 \mathrm{mg})$. For the amino acids Cys $\left(0.10\right.$-Double $50^{\circ} \mathrm{C}$ Couplin (HS)) special program were used. After completion of the automated synthesis the resin was transferred from the reaction vessel in a $10 \mathrm{~mL}-B D$-syringe, washed with $D C M(15 \times 8 \mathrm{~mL})$ and dried under reduced pressure. A small amount of the peptide $\mathbf{5 0}$ was cleaved from the resin and completely deprotected according to GSP 3 (chapter 8.4.3) by using $250 \mu \mathrm{L}$ of cleavage mixture containing EDT. The rest of the resin was used for labelling reactions (peptide 53 and peptide 56).

ESI-MS(+) $(m / z(\%)): 821.7(85)[\mathrm{M}+5 \mathrm{H}]^{5+}, 1027.1(100)[\mathrm{M}+4 \mathrm{H}]^{4+}, 1368.8(22)$ $[\mathrm{M}+3 \mathrm{H}]^{3+}, 4100.4(100)[\mathrm{M}]$ (deconvoluted).

HR-MS (ESI):

calculated for $\left[\mathrm{C}_{200} \mathrm{H}_{326} \mathrm{~N}_{47} \mathrm{O}_{39} \mathrm{~S}_{3}\right]^{5+}\left([\mathrm{M}+5 \mathrm{H}]^{5+}\right)$ : 821.6832; found: 821.6826,

calculated for $\left[\mathrm{C}_{200} \mathrm{H}_{325} \mathrm{~N}_{47} \mathrm{O}_{39} \mathrm{~S}_{3}\right]^{4+}\left([\mathrm{M}+4 \mathrm{H}]^{4+}\right): 1026.8522$; found: 1026.8509 ,

calculated for $\left[\mathrm{C}_{200} \mathrm{H}_{321} \mathrm{~N}_{47} \mathrm{O}_{39} \mathrm{~S}_{3}\right][\mathrm{M}]:$ 4101.4; found: 4101.4 (deconvoluted). 


\subsubsection{Synthesis of VAMP-8 (63 - 100) Rattus norvegicus (51)}

H-KVARKFWWKNVKMIVIICVIVLIIVILIILFATGTIPT-OH

51

$\mathrm{C}_{215} \mathrm{H}_{358} \mathrm{~N}_{48} \mathrm{O}_{43} \mathrm{~S}_{2}$

$4364.6 \mathrm{~g} / \mathrm{mol}$

Peptide 51 was synthesised according to GSP 2 (chapter 8.4.2) by using the CEM LibertyBlue peptide synthesizer. For the synthesis the batch size of $0.10 \mathrm{mmol}$ with douple coupling procedure (0.10-Double-Coupling (HS)) was used as well as the Fmoc- $\mathrm{Thr}(\mathrm{O} t \mathrm{Bu})$-WANG resin $\mathrm{LL}(0.35 \mathrm{mmol} / \mathrm{g}, 286 \mathrm{mg})$. For the amino acids Cys $\left(0.10\right.$-Double $50^{\circ} \mathrm{C}$ Couplin (HS)) and Arg (0.10-Double Arg Coupling (HS)) special programs were used. After completion of the automated synthesis the resin was transferred from the reaction vessel in a $10 \mathrm{~mL}-B D$-syringe, washed with DCM $(15 \times 8 \mathrm{~mL})$ and dried under reduced pressure. A small amount of the peptide $\mathbf{5 1}$ was cleaved from the resin and completely deprotected according to GSP 3 (chapter 8.4.3) by using $500 \mu \mathrm{L}$ of cleavage mixture containing EDT. The rest of the resin was used for labelling reactions (peptide 54 and peptide 57 ).

ESI-MS(+) $(m / z(\%)): 874.4(28)[\mathrm{M}+5 \mathrm{H}]^{5+}, 1092.9(14)[\mathrm{M}+4 \mathrm{H}]^{4+}, 1456.6(7)[\mathrm{M}+3 \mathrm{H}]^{3+}$, 4364.6 (11) [M] (deconvoluted).

HR-MS (ESI):

calculated for $\left[\mathrm{C}_{215} \mathrm{H}_{362} \mathrm{~N}_{48} \mathrm{O}_{43} \mathrm{~S}_{2}\right]^{4+}\left([\mathrm{M}+4 \mathrm{H}]^{4+}\right)$ : 1092.9283; found: 1092.9281, calculated for $\left[\mathrm{C}_{215} \mathrm{H}_{358} \mathrm{~N}_{48} \mathrm{O}_{43} \mathrm{~S}_{2}\right][\mathrm{M}]:$ 4364.7; found: 4364.6 (deconvoluted). 


\subsubsection{Synthesis of hybrid construct VAMPext (52)}

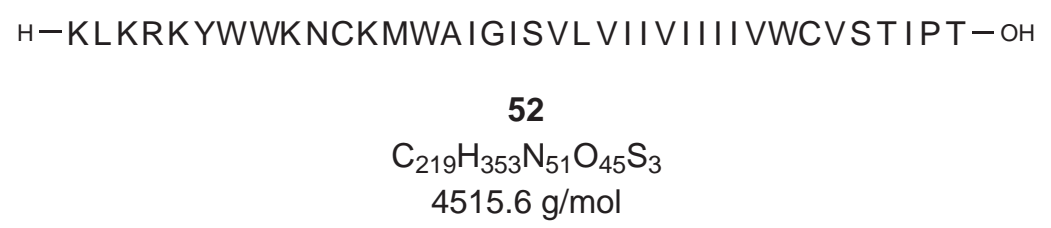

Peptide 52 was synthesised according to GSP 2 (chapter 8.4.2) by using the CEM LibertyBlue peptide synthesizer. For the synthesis the batch size of $0.10 \mathrm{mmol}$ with douple coupling procedure (0.10-Double-Coupling (HS)) was used as well as the Fmoc-Thr(OtBu)-WANG resin $\mathrm{LL}(0.35 \mathrm{mmol} / \mathrm{g}, 286 \mathrm{mg})$. For the amino acids Cys $\left(0.10\right.$-Double $50^{\circ} \mathrm{C}$ Couplin (HS)) special program were used. After completion of the automated synthesis the resin was transferred from the reaction vessel in a $10 \mathrm{~mL}-B D$-syringe, washed with DCM $(15 \times 8 \mathrm{~mL})$ and dried under reduced pressure. A small amount of the peptide $\mathbf{5 2}$ was cleaved from the resin and completely deprotected according to GSP 3 (chapter 8.4.3) by using $500 \mu \mathrm{L}$ of cleavage mixture containing EDT. The rest of the resin was used for labelling reactions (peptide 55).

ESI-MS(+) $(m / z(\%)): 904.1(12)[\mathrm{M}+5 \mathrm{H}]^{5+}, 1130.2(20)[\mathrm{M}+4 \mathrm{H}]^{4+}, 4515.6(19)[\mathrm{M}]$ (deconvoluted).

HR-MS (ESI):

calculated for $\left[\mathrm{C}_{219} \mathrm{H}_{358} \mathrm{~N}_{51} \mathrm{O}_{45} \mathrm{~S}_{3}\right]^{5+}\left([\mathrm{M}+5 \mathrm{H}]^{5+}\right)$ : 904.1; found: 904.1, calculated for $\left[\mathrm{C}_{219} \mathrm{H}_{357} \mathrm{~N}_{51} \mathrm{O}_{45} \mathrm{~S}_{3}\right]^{4+}\left([\mathrm{M}+4 \mathrm{H}]^{4+}\right): 1130.2$; found: 1130.2 , calculated for $\left[\mathrm{C}_{219} \mathrm{H}_{353} \mathrm{~N}_{51} \mathrm{O}_{45} \mathrm{~S}_{3}\right][\mathrm{M}]:$ 4515.6; found: 4515.6 (deconvoluted). 


\subsubsection{Synthesis of Atto647N-labelled VAMP-3 (70 - 100)+CVS Rattus norvegicus (53)}

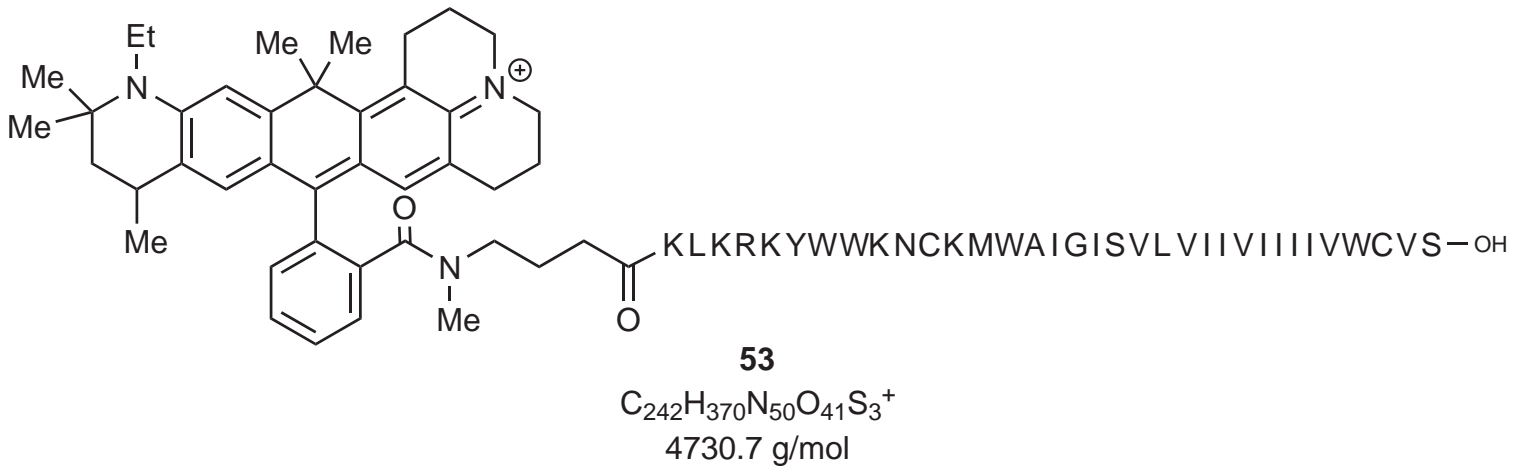

The resin-bound peptide 50 (chapter 8.9.14) was swollen in NMP $(2.00 \mathrm{~mL}$ ) for $1 \mathrm{~h}$ and to remove the final Fmoc-protecting group piperidine $(20 \%)$ in NMP $(2 \times 1.00 \mathrm{~mL})$ was added and shaken for $2 \times 15 \mathrm{~min}$. The resin was washed with NMP $(5 \times 2.00 \mathrm{~mL}), \mathrm{DCM}(5 \times 2.00 \mathrm{~mL})$ and NMP $(5 \times 2.00 \mathrm{~mL})$. Afterwards, a solution of Atto647N-NHS-ester $(1.00 \mathrm{mg}, 1.19 \mu \mathrm{mol}$, 1.00 eq.) and DIPEA $(10.0 \mu \mathrm{L}, 51.8 \mu \mathrm{mol}, 44.0$ eq. $)$ in NMP $(200 \mu \mathrm{L})$ was added to the resin-bound peptide 50 (17.1 mg, $2.37 \mu \mathrm{mol}, 2.00$ eq.) and shaken overnight under light exclusion. The solution was filtrated and the resin was washed with NMP $(5 \times 2.00 \mathrm{~mL})$ and DCM $(10 \times 2.00 \mathrm{~mL})$ and dried under reduced pressure. The peptide $\mathbf{5 3}$ was cleaved from the resin and completely deprotected according to GSP 3 (chapter 8.4.3) by using EDT containing cleavage mixture $(500 \mu \mathrm{L})$. After precipitation and drying under reduced pressure, the crude peptide was suspended in $\mathrm{H}_{2} \mathrm{O} / \mathrm{MeCN}$ and lyophilised to obtain peptide $\mathbf{5 3}$ as a dark blue solid.

ESI-MS(+) $(m / z(\%)): 947.4(23)[\mathrm{M}+5 \mathrm{H}]^{5+}, 1183.9(14)[\mathrm{M}+4 \mathrm{H}]^{4+}, 4630.7(18)[\mathrm{M}]$ (deconvoluted).

HR-MS (ESI):

calculated for $\left[\mathrm{C}_{242} \mathrm{H}_{375} \mathrm{~N}_{50} \mathrm{O}_{41} \mathrm{~S}_{3}\right]^{5+}\left([\mathrm{M}+5 \mathrm{H}]^{5+}\right)$ : 947.1597; found: 947.1590, calculated for $\left[\mathrm{C}_{242} \mathrm{H}_{374} \mathrm{~N}_{50} \mathrm{O}_{41} \mathrm{~S}_{3}\right]^{4+}\left([\mathrm{M}+4 \mathrm{H}]^{4+}\right)$ : 1183.9489; found: 1183.9467, calculated for $\left[\mathrm{C}_{242} \mathrm{H}_{370} \mathrm{~N}_{50} \mathrm{O}_{41} \mathrm{~S}_{3}\right.$ ] [M]: 4730.7; found: 4730.7 (deconvoluted). 


\subsubsection{Synthesis of Atto647N-labelled VAMP-8 (63 - 100) Rattus norvegicus (54)}

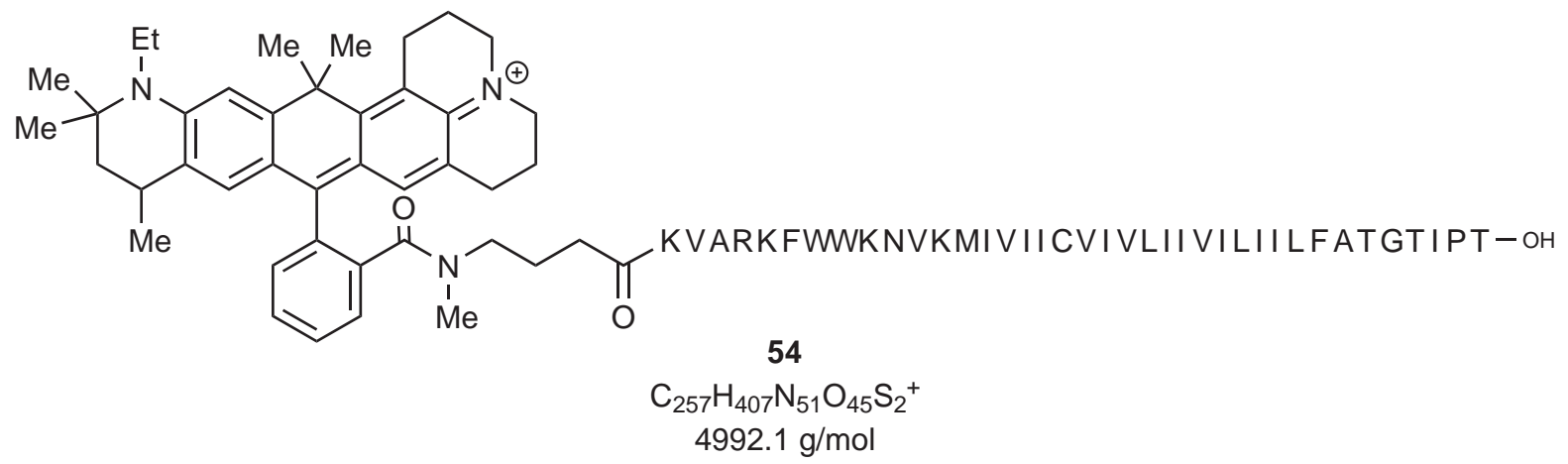

The resin-bound peptide 51 (chapter 8.9.15) was swollen in NMP $(2.00 \mathrm{~mL}$ ) for $1 \mathrm{~h}$ and to remove the final Fmoc-protecting group piperidine $(20 \%)$ in NMP $(2 \times 1.00 \mathrm{~mL})$ was added and shaken for $2 \times 15 \mathrm{~min}$. The resin was washed with NMP $(5 \times 2.00 \mathrm{~mL}), \mathrm{DCM}(5 \times 2.00 \mathrm{~mL})$ and NMP $(5 \times 2.00 \mathrm{~mL})$. Afterwards, a solution of Atto647N-NHS-ester $(1.00 \mathrm{mg}, 1.19 \mu \mathrm{mol}$, 1.00 eq.) and DIPEA $(10.0 \mu \mathrm{L}, 51.8 \mu \mathrm{mol}, 44.0$ eq. $)$ in NMP $(200 \mu \mathrm{L})$ was added to the resin-bound peptide 51 (14.8 mg, $2.37 \mu \mathrm{mol}, 2.00 \mathrm{eq}$.) and shaken overnight under light exclusion. The solution was filtrated and the resin was washed with NMP $(5 \times 2.00 \mathrm{~mL})$ and DCM $(10 \times 2.00 \mathrm{~mL})$ and dried under reduced pressure. The peptide $\mathbf{5 4}$ was cleaved from the resin and completely deprotected according to GSP 3 (chapter 8.4.3) by using EDT containing cleavage mixture $(1.00 \mathrm{~mL})$. After precipitation and drying under reduced pressure, the crude peptide was suspended in $\mathrm{H}_{2} \mathrm{O} / \mathrm{MeCN}$ and lyophilised to obtain peptide $\mathbf{5 4}$ as a dark blue solid.

$\operatorname{ESI-MS}(+)(\mathrm{m} / \mathrm{z}(\%)): 1249.8(11)[\mathrm{M}+4 \mathrm{H}]^{4+}$.

\section{HR-MS (ESI):}

calculated for $\left[\mathrm{C}_{257} \mathrm{H}_{412} \mathrm{~N}_{51} \mathrm{O}_{45} \mathrm{~S}_{2}\right]^{5+}\left([\mathrm{M}+5 \mathrm{H}]^{5+}\right)$ : 1000.2212 ; found: 1000.2210 , calculated for $\left[\mathrm{C}_{257} \mathrm{H}_{411} \mathrm{~N}_{51} \mathrm{O}_{45} \mathrm{~S}_{2}\right]^{4+}\left([\mathrm{M}+4 \mathrm{H}]^{4+}\right): 1249.0215$; found: 1249.0185 , calculated for $\left[\mathrm{C}_{257} \mathrm{H}_{407} \mathrm{~N}_{51} \mathrm{O}_{45} \mathrm{~S}_{2}\right][\mathrm{M}]:$ 4992.1; found: 4992.1 (deconvoluted). 


\subsubsection{Synthesis of Atto647N-labelled hybrid construct VAMPext}

(55)

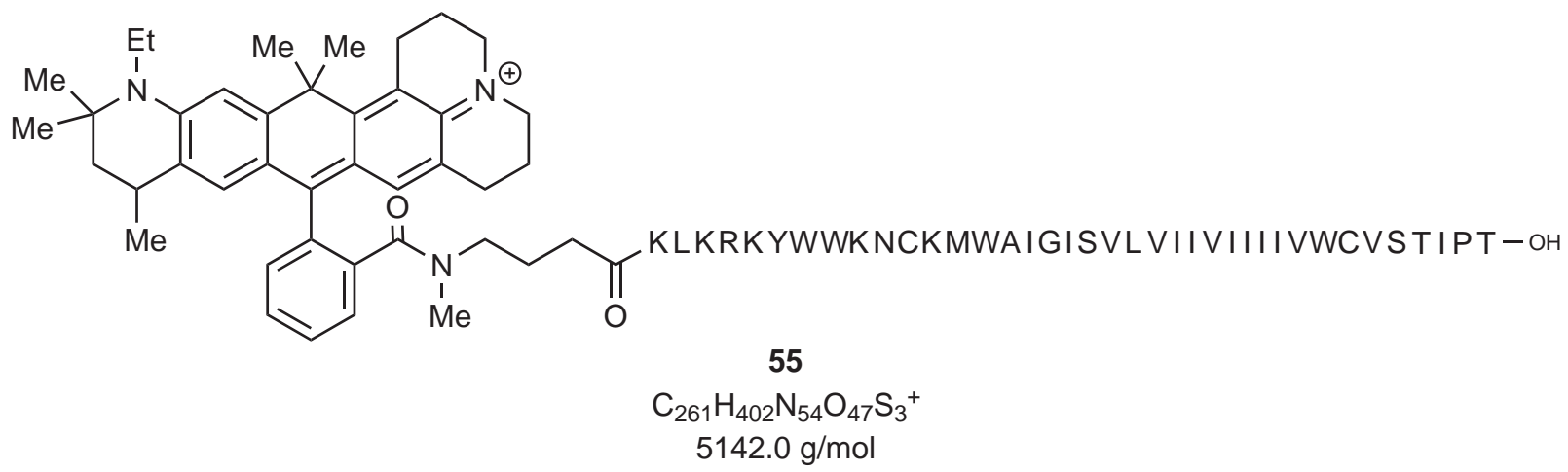

The resin-bound peptide 52 (chapter 8.9.16) was swollen in NMP $(2.00 \mathrm{~mL}$ ) for $1 \mathrm{~h}$ and to remove the final Fmoc-protecting group piperidine $(20 \%)$ in NMP $(2 \times 1.00 \mathrm{~mL})$ was added and shaken for $2 \times 15 \mathrm{~min}$. The resin was washed with NMP $(5 \times 2.00 \mathrm{~mL}), \mathrm{DCM}(5 \times 2.00 \mathrm{~mL})$ and NMP $(5 \times 2.00 \mathrm{~mL})$. Afterwards, a solution of Atto647N-NHS-ester $(1.00 \mathrm{mg}, 1.19 \mu \mathrm{mol}$, 1.00 eq.) and DIPEA $(10.0 \mu \mathrm{L}, 51.8 \mu \mathrm{mol}, 44.0$ eq. $)$ in NMP $(150 \mu \mathrm{L})$ was added to the resin-bound peptide 52 (16.5 mg, $2.37 \mu \mathrm{mol}, 2.00$ eq.) and shaken overnight under light exclusion. The solution was filtrated and the resin was washed with NMP $(5 \times 2.00 \mathrm{~mL})$ and DCM $(10 \times 2.00 \mathrm{~mL})$ and dried under reduced pressure. The peptide $\mathbf{5 5}$ was cleaved from the resin and completely deprotected according to GSP 3 (chapter 8.4.3) by using EDT containing cleavage mixture $(500 \mu \mathrm{L})$. After precipitation and drying under reduced pressure, the crude peptide was suspended in $\mathrm{H}_{2} \mathrm{O} / \mathrm{MeCN}$ and lyophilised to obtain peptide $\mathbf{5 5}$ as a dark blue solid.

ESI-MS(+) $(m / z(\%)): 858.2(9)[\mathrm{M}+6 \mathrm{H}]^{6+}, 1029.6(24)[\mathrm{M}+5 \mathrm{H}]^{5+}, 5142.0(11)[\mathrm{M}]$ (deconvoluted).

HR-MS (ESI):

calculated for $\left[\mathrm{C}_{261} \mathrm{H}_{408} \mathrm{~N}_{54} \mathrm{O}_{47} \mathrm{~S}_{3}\right]^{6+}\left([\mathrm{M}+6 \mathrm{H}]^{6+}\right)$ : 858.2; found: 858.2, calculated for $\left[\mathrm{C}_{261} \mathrm{H}_{407} \mathrm{~N}_{54} \mathrm{O}_{47} \mathrm{~S}_{3}\right]^{5+}\left([\mathrm{M}+5 \mathrm{H}]^{5+}\right)$ : 1029.6; found: 1029.6 , calculated for $\left[\mathrm{C}_{261} \mathrm{H}_{402} \mathrm{~N}_{54} \mathrm{O}_{47} \mathrm{~S}_{3}\right]$ [M]: 5142.0; found: 5142.0 (deconvoluted). 


\subsubsection{Synthesis of RhodamineRed-labelled VAMP-3 (70 - 100)+CVS Rattus norvegicus (56)}

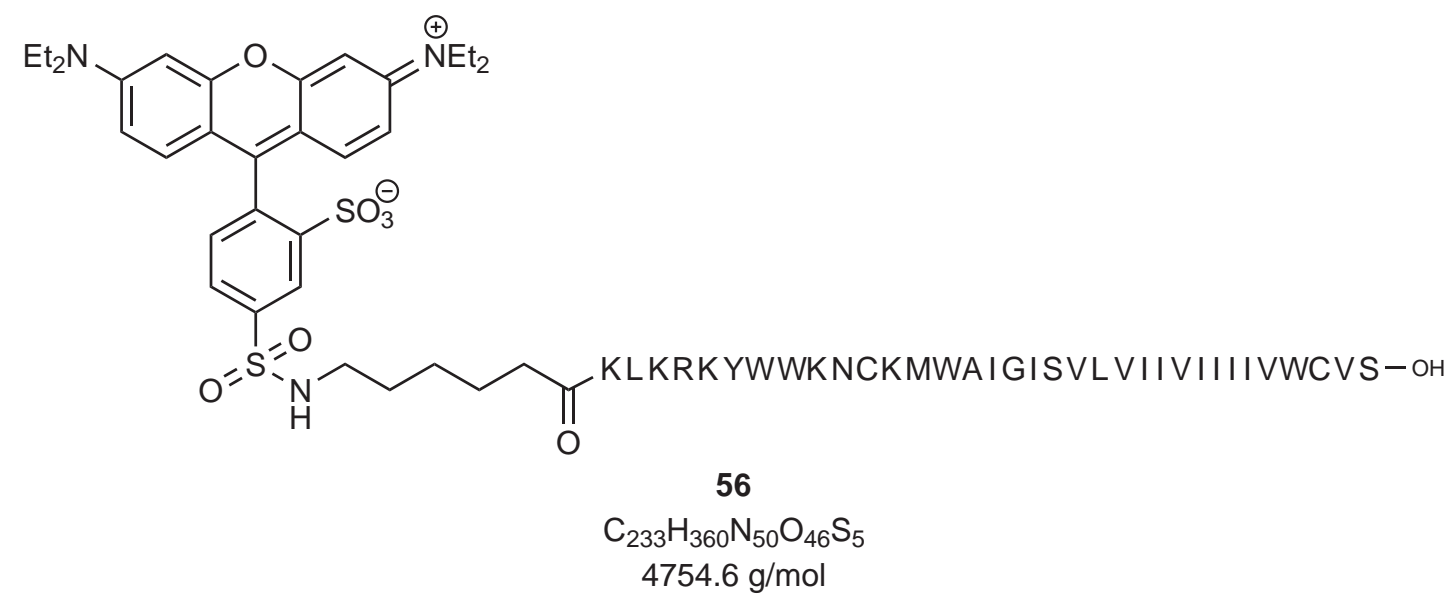

The resin-bound peptide 50 (chapter 8.9.14) was swollen in NMP $(2.00 \mathrm{~mL}$ ) for $1 \mathrm{~h}$ and to remove the final Fmoc-protecting group piperidine $(20 \%)$ in NMP $(2 \times 1.00 \mathrm{~mL})$ was added and shaken for $2 \times 15 \mathrm{~min}$. The resin was washed with NMP $(5 \times 2.00 \mathrm{~mL}), \mathrm{DCM}(5 \times 2.00 \mathrm{~mL})$ and NMP $(5 \times 2.00 \mathrm{~mL})$. Afterwards, a solution of RhodamineRed ${ }^{\mathrm{TM}}$-succinimidylester $(1.00 \mathrm{mg}$, $1.30 \mu \mathrm{mol}, 1.00$ eq.) and DIPEA $(10.0 \mu \mathrm{L}, 60.0 \mu \mathrm{mol}, 46.2$ eq. $)$ in NMP $(150 \mu \mathrm{L})$ was added to the resin-bound peptide 50 (19.2 mg, $2.60 \mu \mathrm{mol}, 2.00$ eq.) and shaken overnight under light exclusion. The solution was filtrated and the resin was washed with NMP $(5 \times 2.00 \mathrm{~mL})$ and DCM $(10 \times 2.00 \mathrm{~mL})$ and dried under reduced pressure. The peptide $\mathbf{5 6}$ was cleaved from the resin and completely deprotected according to GSP 3 (chapter 8.4.3) by using EDT containing cleavage mixture $(500 \mu \mathrm{L})$. After precipitation and drying under reduced pressure, the crude peptide was suspended in $\mathrm{H}_{2} \mathrm{O} / \mathrm{MeCN}$ and lyophilised to obtain peptide $\mathbf{5 6}$ as a pink solid.

ESI-MS(+) $(m / z(\%)): 952.5(9)[\mathrm{M}+5 \mathrm{H}]^{5+}, 1190.7(10)[\mathrm{M}+4 \mathrm{H}]^{4+}, 4754.6$ (8) [M] (deconvoluted).

HR-MS (ESI):

calculated for $\left[\mathrm{C}_{233} \mathrm{H}_{365} \mathrm{~N}_{50} \mathrm{O}_{46} \mathrm{~S}_{5}\right]^{5+}\left([\mathrm{M}+5 \mathrm{H}]^{5+}\right)$ : 952.5; found: 952.5 ,

calculated for $\left[\mathrm{C}_{233} \mathrm{H}_{364} \mathrm{~N}_{50} \mathrm{O}_{46} \mathrm{~S}_{5}\right]^{4+}\left([\mathrm{M}+4 \mathrm{H}]^{4+}\right)$ : 1190.7; found: 1190.7 ,

calculated for $\left[\mathrm{C}_{233} \mathrm{H}_{360} \mathrm{~N}_{50} \mathrm{O}_{46} \mathrm{~S}_{5}\right]$ ([M]): 4754.6; found: 4754.6 (deconvoluted). 


\subsubsection{Synthesis of RhodamineRed-labelled VAMP-8 (63 - 100) Rattus norvegicus (57)}

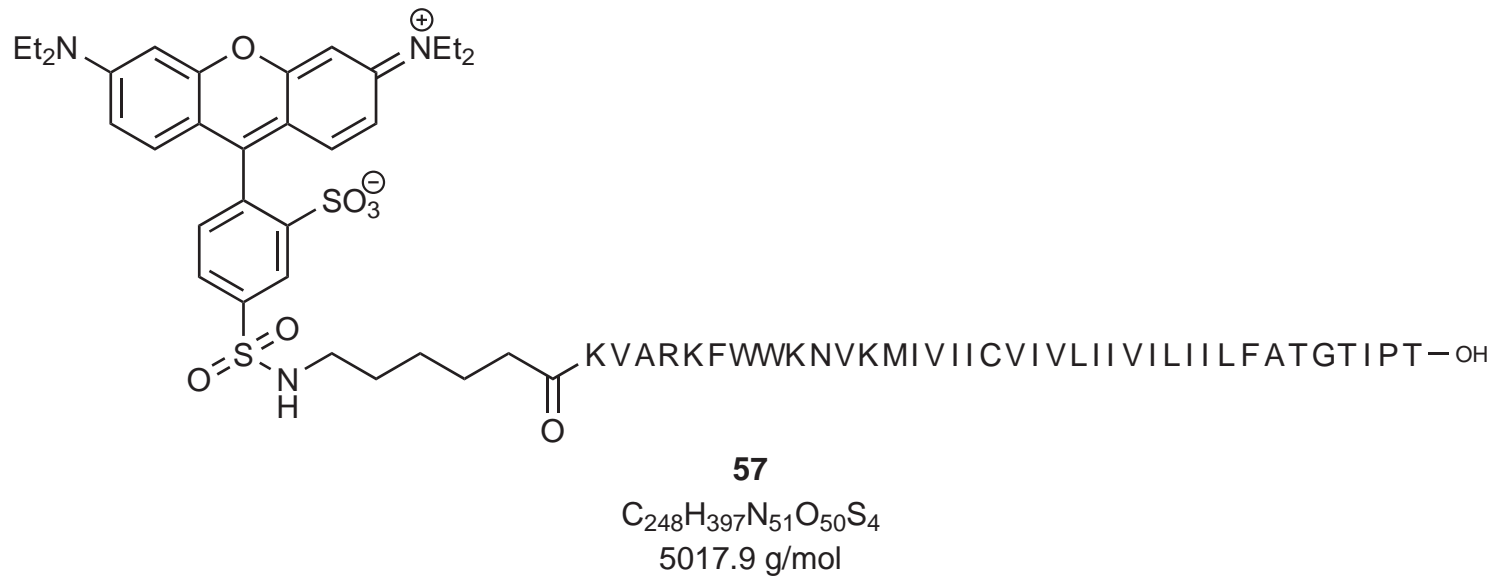

The resin-bound peptide 51 (chapter 8.9.15) was swollen in NMP $(2.00 \mathrm{~mL}$ ) for $1 \mathrm{~h}$ and to remove the final Fmoc-protecting group piperidine $(20 \%)$ in NMP $(2 \times 1.00 \mathrm{~mL})$ was added and shaken for $2 \times 15 \mathrm{~min}$. The resin was washed with NMP $(5 \times 2.00 \mathrm{~mL}), \mathrm{DCM}(5 \times 2.00 \mathrm{~mL})$ and NMP $(5 \times 2.00 \mathrm{~mL})$. Afterwards, a solution of RhodamineRed ${ }^{\mathrm{TM}}$-succinimidylester $(1.00 \mathrm{mg}$, $1.30 \mu \mathrm{mol}, 1.00$ eq.) and DIPEA $(10.0 \mu \mathrm{L}, 60.0 \mu \mathrm{mol}, 46.2$ eq. $)$ in NMP $(200 \mu \mathrm{L})$ was added to the resin-bound peptide 51 (16.2 $\mathrm{mg}, 2.60 \mu \mathrm{mol}, 2.00$ eq.) and shaken overnight under light exclusion. The solution was filtrated and the resin was washed with NMP $(5 \times 2.00 \mathrm{~mL})$ and DCM $(10 \times 2.00 \mathrm{~mL})$ and dried under reduced pressure. The peptide $\mathbf{5 7}$ was cleaved from the resin and completely deprotected according to GSP 3 (chapter 8.4.3) by using EDT containing cleavage mixture $(1.00 \mathrm{~mL})$. After precipitation and drying under reduced pressure, the crude peptide was suspended in $\mathrm{H}_{2} \mathrm{O} / \mathrm{MeCN}$ and lyophilised to obtain peptide $\mathbf{5 7}$ as a pink solid.

ESI-MS(+) $(m / z(\%)): 1005.2(17)[\mathrm{M}+5 \mathrm{H}]^{5+}, 1256.5(19)[\mathrm{M}+4 \mathrm{H}]^{4+}, 5017.9(16)[\mathrm{M}]$ (deconvoluted).

HR-MS (ESI):

calculated for $\left[\mathrm{C}_{248} \mathrm{H}_{402} \mathrm{~N}_{51} \mathrm{O}_{50} \mathrm{~S}_{4}\right]^{5+}\left([\mathrm{M}+5 \mathrm{H}]^{5+}\right)$ : 1005.1887 ; found: 1005.1853 , calculated for $\left[\mathrm{C}_{248} \mathrm{H}_{401} \mathrm{~N}_{51} \mathrm{O}_{50} \mathrm{~S}_{4}\right]^{4+}\left([\mathrm{M}+4 \mathrm{H}]^{4+}\right)$ : 1256.4848; found: 1256.4863 , calculated for $\left[\mathrm{C}_{248} \mathrm{H}_{397} \mathrm{~N}_{51} \mathrm{O}_{50} \mathrm{~S}_{4}\right]$ ([M]): 5017.9; found: 5017.9 (deconvoluted). 


\section{Abbreviations}

\begin{tabular}{|c|c|}
\hline$\Phi$ & Quantum yield \\
\hline$\alpha-\mathrm{CNV}$ & $\alpha$-Carboxy-6-nitroveratryl \\
\hline$\alpha$-SNAP & $\alpha$-soluble NSF attachment protein \\
\hline$\delta$ & Chemical shift \\
\hline$\delta_{\mathrm{a}}$ & Two-photon cross-section \\
\hline$\delta_{\mathrm{u}}$ & Two-photon action cross-section \\
\hline$\lambda_{\max }$ & Wavelength of maximal absorption \\
\hline$\varepsilon$ & Extinction coefficient \\
\hline [M] & Molecule peak (deconvoluted) \\
\hline$B D$ & Becton Dickinson Discardit ${ }^{T M} \|$ \\
\hline H.s. & Homo sapiens \\
\hline$J$ & Coupling constant \\
\hline R.n. & Rattus norvegicus \\
\hline$m / z$ & Mass to charge ratio \\
\hline$p-\mathrm{NPCl}$ & $p$-Nitrophenyl chloroformate \\
\hline$t \mathrm{Bu}$ & tert-Butyle \\
\hline$t_{\mathrm{R}, \text { netto }}$ & Netto retention time \\
\hline$t_{\mathrm{R}}$ & Retention time \\
\hline M & $\mathrm{mol} / \mathrm{L}$ \\
\hline$A$ & Alanine \\
\hline aa & Amino acid \\
\hline $\mathrm{AAA}+$ & ATPases associated with various cellular activities \\
\hline $\mathrm{Ac}_{2} \mathrm{O}$ & Acetic acid anhydride \\
\hline $\mathrm{AcOH}$ & Acetic acid \\
\hline Ad & Adamantyl \\
\hline ADP & Adenosine diphosphate \\
\hline Ala & Alanine \\
\hline
\end{tabular}




\begin{tabular}{|c|c|}
\hline AMPPNP & Adenosine 5'-( $\beta, \gamma$-imido $)$ triphosphate \\
\hline $\operatorname{Arg}$ & Arginine \\
\hline Asn & Asparagine \\
\hline Asp & Aspartic acid \\
\hline ATP & Adenosine triphosphate \\
\hline Boc & tert-Butoxycarbonyle \\
\hline $\mathrm{C}$ & Cysteine \\
\hline CATCHR & Complex associated with tethering containing helical rods \\
\hline CDNI & 4-Carboxymethoxy-7-nitroindolinyl \\
\hline CHAPS & 3-[(3-Cholamidopropyl)dimethylammonio]-1-propanesulfonate \\
\hline $\mathrm{CM}$ & Coumarin-4-ylmethyl \\
\hline COSY & Correlation spectroscopy \\
\hline Cpx & Complexin \\
\hline $\mathrm{Cpx} \mathrm{OG}^{\mathrm{OG}}$ & Complexin I (1 - 134) E39C, C105S, Oregon-Green-labeled \\
\hline Cys & Cysteine \\
\hline $\mathrm{D}$ & Aspartic acid \\
\hline$d$ & Doublet \\
\hline $\mathrm{D}(\mathrm{DMNPB})$ & Fmoc-Asp(DMNPB)-OH (27) \\
\hline D* & Caged aspartic acid \\
\hline DCC & $N, N^{\prime}$-Dicyclohexylcarbodiimide \\
\hline DCM & Dichloromethane \\
\hline Ddz & 3,5-Dimethoxy- $\alpha, \alpha$-dimethylbenzyloxycarbonyl \\
\hline DEACM & 7-(Diethylamino)coumarin-4-yl)methyl \\
\hline DEACM450 & $\begin{array}{l}\text { (E)-2-(3-(7-Diethylamino)-4-(hydroxymethyl)-2-oxo-2H-chromen-3-yl)acryl- } \\
\text { amido)succinate }\end{array}$ \\
\hline DHPC & Dihexanoylphosphatidylcholine \\
\hline DIC & $N, N^{\prime}$-Diisopropylcarbodiimide \\
\hline DIPEA & Diisopropylethylamine \\
\hline DMAP & 4-Dimethylaminopyridine \\
\hline DMCM & 6,7-Dimethoxy(coumarin-4-yl)methyl \\
\hline DMF & Dimethylformamide \\
\hline DMNPB & 3-(4,5-dimethoxy-2-nitrophenyl)-2-butyl \\
\hline DMPC & Dimyristoylphosphatidylcholine \\
\hline
\end{tabular}




\begin{tabular}{|c|c|}
\hline DMSO & Dimethylsulfoxide \\
\hline Dni & 5,7-Dinitroindolinyl \\
\hline DPC & Dodecylphosphocholine \\
\hline DTT & Dithiothreitol \\
\hline$E$ & Glutamic acid \\
\hline EDT & Ethanedithiole \\
\hline EDTA & Ethylenediaminetetraacetic acid \\
\hline EM & Electron microscopy \\
\hline EPL & Expressed protein ligation \\
\hline eq & Equivalent(s) \\
\hline ESI & Electron spray ionisation \\
\hline $\mathrm{Et}_{2} \mathrm{O}$ & Diethylether \\
\hline EtOAc & Ethylacetate \\
\hline $\mathrm{F}$ & Phenylalanine \\
\hline Fmoc & 9-Fluorenylmethoxycarbonyl \\
\hline FRET & Fluorescence resonance energy transfer \\
\hline G & Glycine \\
\hline Gln & Glutamine \\
\hline Glu & Glutamic acid \\
\hline Gly & Glycine \\
\hline GM & GOEPPERT-MAYER unit \\
\hline $\mathrm{H}_{2} \mathrm{O}$ & Water \\
\hline HATU & 2-(1H-7-Azabenzotriazole-1-yl)-1,1,3,3-tetramethyluronium-hexafluorophosphate \\
\hline HBTU & 2-(1H-Benzotriazol-1-yl)-1,1,3,3,-tetramethyluronium-hexafluorophosphate \\
\hline HEPES & 4-(2-hydroxyethyl)-1-piperazineethynesulfonic acid \\
\hline HMBC & Heteronuclear multiple bond correlation \\
\hline HOAt & 1-Hydroxy-7-azabenzotriazole \\
\hline HOBt & 1-Hydroxybenzotriazole \\
\hline HPLC & High performance liquid chromatography \\
\hline HR-MS & High resolution mass spectrometry \\
\hline HS & High-swelling \\
\hline HSQC & Heteronuclear single quantum coherence \\
\hline I & Isoleucine \\
\hline
\end{tabular}


Ile

IR

Isoleucine

ITC

Infrared

ITC

Isothermal titration calorimetry

$\mathrm{K}$

Lysine

$\mathrm{K}(\mathrm{Nvoc})$

Fmoc-Lys(Nvoc)-OH (16)

$\mathrm{K}^{*}$

Caged lysine

$\mathrm{KAc}$

Potassium acetate

KGlu

Potassium glutamate

L

Leucine

Leu

Leucine

LL

Low loaded resin

Lys

Lysine

M

Methionine

$\mathrm{m}$

Multiplet

$\mathrm{MeCN}$

Acetonitrile

Met

Methionine

MS

Mass spectrometry

$\mathrm{N}$

Asparagine

$\mathrm{N}(\mathrm{Ad})$

Fmoc-Asp(Ad)-OH (4)

NaCholate

Sodium cholate

NB

o-Nitrobenzyl

Nboc

o-Nitrobenzyloxycarbonyl

NMP

$N$-methyl-2-pyrrolidinone

NMR

Nuclear magnetic resonance spectroscopy

NSF

$N$-ethylmaleimide-sensitive factor

NV

6-Nitroveratryl

$\mathrm{NV}-\mathrm{OH}$

6-Nitroveratrylalcohol

Nvoc

6-Nitroveratryloxycarbonyl

Nvoc-Cl

6-Nitroveratryl chloroformate

Nycodenz ${ }^{\circledR}$

1-N,3-N-Bis(2,3-dihydroxypropyl)-5-[N-(2,3-dihydroxypropyl)acetamido]-2,4,6triiodobenzene-1,3-dicarboxamide

$\mathrm{OtBu}$ tert-Butylate

OctGlu

Octyl $\beta$-D-glucopyranoside 
OG

Oxyma

$\mathrm{Pbf}$

PC

PE

Phe

$\mathrm{PIP}_{2}$

POPC

POPS

PPG

$\mathrm{PPh}_{3}$

PS

PyBOP

Q

q

$\mathrm{R}$

$\mathrm{R}(\mathrm{Nvoc})$

$\mathrm{R}^{*}$

RhoRed

RIM

RIM-BP

RP

S

$\mathrm{s}$

$\mathrm{Sbr}$

SDS-PAGE

Ser

SM

SN25

SNAP-25

SNARE

SPPS

SUV
Oregon Green $₫ 488$

OxymaPure ${ }^{\circledR}$, Ethyl(hydroxyimino)cyanoacetate

2,2,4,6,7-Pentamethyl-dihydrobenzofurane-5-sulfonyle

Phosphatidylcholine

Phosphatidylethanolamine

Phenylalanine

Phosphatidylinositol 4,5-biphosphate

Palmitoyl-oleyl-sn-phosphatidylcholine

Palmitoyl-oleyl-sn-phosphatidylserine

Photocleavable protecting group

Triphenylphosphine

Phosphatidylserine

Benzotriazol-1-yl-oxytripyrrolidinophosphonium hexafluorophosphate

Glutamine

Quartet

Arginine

Fmoc-Arg(Nvoc)-OH (21)

Caged arginine

Rhodamine Red ${ }^{\mathrm{TM}}$

Rab3-interacting molecule

RIM-binding protein

Reversed phase

Serine

Singlet

Broad singlet

Sodium dodecylsulfate polyacrylamide gel electrophoresis

Serine

Sec1/Munc18-like

25 kDa synaptosomal-associated protein

25 kDa synaptosomal-associated protein

Soluble NSF attachment protein receptor

Solid phase peptide synthesis

Small unilamellar vesicle 


\begin{tabular}{ll} 
Syb & Synaptobrevin \\
Syx & Syntaxin \\
T & Threonine \\
$\mathrm{t}$ & Triplet \\
t-SNARE & Target-SNARE \\
TCEP & Tris(2-carboxyethyl)phosphine \\
TFA & Trifluoroacetic acid \\
THF & Tetrahydrofurane \\
Thr & Threonine \\
TIS & Triisopropylsilane \\
TLC & Thin layer chromatography \\
TMD & Transmembrane domain \\
TMS & Trimethylsilane \\
Trt & Trityle \\
Tyr & Tyrosine \\
UV & Ultraviolet \\
V & Valine \\
v-SNARE & Vesicle-SNARE \\
Val & Valine \\
VAMP & Vesicle-associated membrane protein \\
vis & visible \\
Y & \\
& Tyrosine \\
\hline &
\end{tabular}




\section{Bibliography}

[1] B. Alberts, A. Johnson, J. Lewis, D. Morgan, M. Raff, K. Roberts, P. Walter, Molecular Biology of The Cell, 6. ed., Garland Science, Taylor \& Francis Group, New York, 2015.

[2] D. Nelson, M. Cox, Lehninger Biochemie, 4 ed., Springer-Verlag Berlin, 2009.

[3] R. Jahn, R. H. Scheller, Nat. Rev. Mol. Cell Biol. 2006, 7, 631-643.

[4] T. C. Südhof, J. Rizo, Cold Spring Harbor Perspect. Biol. 2011, 3, a005637.

[5] R. Jahn, D. Fasshauer, Nature 2012, 490, 201-207.

[6] T. Südhof, Neuron 2013, 80, 675-690.

[7] J. Rizo, J. Xu, Annu. Rev. Biophys. 2015, 44, 339-367.

[8] J. Wesolowski, F. Paumet, Virulence 2010, 1, 319-324.

[9] R. B. Sutton, D. Fasshauer, R. Jahn, A. T. Brunger, Nature 1998, 395, 347-353.

[10] P. I. Hanson, J. E. Heuser, R. Jahn, Curr. Opin. Neurobiol. 1997, 7, 310-315.

[11] A. V. Pobbati, A. Stein, D. Fasshauer, Science 2006, 313, 673-676.

[12] K. Wiederhold, D. Fasshauer, J. Biol. Chem. 2009, 284, 13143-13152.

[13] C. Ma, L. Su, A. B. Seven, Y. Xu, J. Rizo, Science 2013, 339, 421-425.

[14] S. Martinez-Arca, S. Arold, R. Rudge, F. Laroche, T. Galli, Traffic 2004, 5, 371-382.

[15] F. Deák, O.-H. Shin, E. T. Kavalali, T. C. Südhof, J. Neurosci. 2006, 26, 6668-6676.

[16] A. M. Walter, K. Wiederhold, D. Bruns, D. Fasshauer, J. B. Sørensen, J. Cell Biol. 2010, 188, 401-413.

[17] K. Wiederhold, T. H. Kloepper, A. M. Walter, A. Stein, N. Kienle, J. B. Sørensen, D. Fasshauer, J. Biol. Chem. 2010, 285, 21549-21559.

[18] J. M. Hernandez, A. Stein, E. Behrmann, D. Riedel, A. Cypionka, Z. Farsi, P. J. Walla, S. Raunser, R. Jahn, Science 2012, 336, 1581-1584. 
[19] F. Li, D. Kümmel, J. Coleman, K. M. Reinisch, J. E. Rothman, F. Pincet, J. Am. Chem. Soc. 2014, 136, 3456-3464.

[20] G. G. Schiavo, F. Benfenati, B. Poulain, O. Rossetto, P. P. de Laureto, B. R. DasGupta, C. Montecucco, Nature 1992, 359, 832-835.

[21] J. Blasi, E. R. Chapman, E. Link, T. Binz, S. Yamasaki, P. D. Camilli, T. C. Südhof, H. Niemann, R. Jahn, Nature 1993, 365, 160-163.

[22] J. Blasi, E. R. Chapman, S. Yamasaki, T. Binz, H. Niemann, R. Jahn, EMBO J. 1993, $12,4821-4828$.

[23] T. H. Söllner, S. W. Whiteheart, M. Brunner, H. Erdjument-Bromage, S. Geromanos, P. Tempst, J. E. Rothman, Nature 1993, 362, 318-324.

[24] A. Stein, G. Weber, M. C. Wahl, R. Jahn, Nature 2009, 460, 525-528.

[25] T. H. Kloepper, C. N. Kienle, D. Fasshauer, Mol. Biol. Cell 2007, 18, 3463-3471.

[26] T. Hayashi, H. McMahon, S. Yamasaki, T. Binz, Y. Hata, T. C. Südhof, H. Niemann, EMBO J. 1994, 13, 5051-5061.

[27] D. Fasshauer, W. Antonin, V. Subramaniam, R. Jahn, Nat. Struct. Mol. Biol. 2002, 9, 144-151.

[28] D. Fasshauer, R. B. Sutton, A. T. Brunger, R. Jahn, PNAS 1998, 95, 15781-15786.

[29] D. Fasshauer, M. Margittai, J. Biol. Chem. 2004, 279, 7613-7621.

[30] J. C. Lerman, J. Robblee, R. Fairman, F. M. Hughson, Biochemistry 2000, 39, 84708479 .

[31] K. M. S. Misura, R. H. Scheller, W. I. Weis, Nature 2000, 404, 355-362.

[32] P. Burkhardt, D. A. Hattendorf, W. I. Weis, D. Fasshauer, EMBO J. 2008, 27, 923-933.

[33] W. S. Trimble, D. M. Cowan, R. H. Scheller, PNAS 1988, 85, 4538-4542.

[34] M. Baumert, P. R. Maycox, F. Navone, P. De Camilli, R. Jahn, EMBO J. 1989, 8, 379-384.

[35] J. E. Rothman, Nature 1994, 372, 55-63.

[36] J. F. Ellena, B. Liang, M. Wiktor, A. Stein, D. S. Cafiso, R. Jahn, L. K. Tamm, PNAS 2009, 106, 20306-20311.

[37] B. Liang, D. Dawidowski, J. F. Ellena, L. K. Tamm, D. S. Cafiso, Biochemistry 2014, 53, 1485-1494. 
[38] J. Burré, M. Sharma, T. Tsetsenis, V. Buchman, M. R. Etherton, T. C. Südhof, Science 2010, 329, 1663-1667.

[39] J. Burré, M. Sharma, T. C. Südhof, J. Neurosci. 2012, 32, 15227-15242.

[40] S. L. Gordon, M. A. Cousin, Traffic 2014, 15, 245-254.

[41] B.-K. Choi, J.-Y. Kim, M.-Y. Cha, I. Mook-Jung, Y.-K. Shin, N. K. Lee, Biochemistry 2015, 54, 1831-1840.

[42] M. Verhage, A. S. Maia, J. J. Plomp, A. B. Brussaard, J. H. Heeroma, H. Vermeer, R. F. Toonen, R. E. Hammer, T. K. van den Berg, M. Missler, H. J. Geuze, T. C. Südhof, Science 2000, 287, 864-869.

[43] F. Varoqueaux, A. Sigler, J.-S. Rhee, N. Brose, C. Enk, K. Reim, C. Rosenmund, PNAS 2002, 99, 9037-9042.

[44] Y. Xu, L. Su, J. Rizo, Biochemistry 2010, 49, 1568-1576.

[45] R. Guan, H. Dai, J. Rizo, Biochemistry 2008, 47, 1474-1481.

[46] K. Weninger, M. E. Bowen, U. B. Choi, S. Chu, A. T. Brunger, Structure 2008, 16, 308-320.

[47] C. Ma, W. Li, Y. Xu, J. Rizo, Nat. Struct. Mol. Biol. 2011, 18, 542-549.

[48] D. W. Wilson, S. W. Whiteheart, M. Wiedmann, M. Brunner, J. E. Rothman, J. Cell Biol. 1992, 117, 531-538.

[49] C. Wimmer, T. M. Hohl, C. A. Hughes, S. A. Müller, T. H. Söllner, A. Engel, J. E. Rothman, J. Biol. Chem. 2001, 276, 29091-29097.

[50] M. Zhao, S. Wu, Q. Zhou, S. Vivona, D. J. Cipriano, Y. Cheng, A. T. Brunger, Nature 2015, 518, 61-67.

[51] M. R. Block, B. S. Glick, C. A. Wilcox, F. T. Wieland, J. E. Rothman, PNAS 1988, 85, 7852-7856.

[52] V. Malhotra, L. Orci, B. S. Glick, M. R. Block, J. E. Rothman, Cell 1988, 54, 221-227.

[53] M. Zhao, A. T. Brunger, J. Mol. Biol. 2016, 428, 1912-1926.

[54] P. I. Hanson, S. W. Whiteheart, Nat. Rev. Mol. Cell Biol. 2005, 6, 519-529.

[55] L.-F. Chang, S. Chen, C.-C. Liu, X. Pan, J. Jiang, X.-C. Bai, X. Xie, H.-W. Wang, S.-F. Sui, Nat. Struct. Mol. Biol. 2012, 19, 268-275. 
[56] R. C. Yu, R. Jahn, A. T. Brunger, Mol. Cell 1999, 4, 97-107.

[57] R. C. Yu, P. I. Hanson, R. Jahn, A. T. Brunger, Nat. Struct. Mol. Biol. 1998, 5, 803-811.

[58] L. M. Rice, A. T. Brunger, Mol. Cell 1999, 4, 85-95.

[59] U. Winter, X. Chen, D. Fasshauer, J. Biol. Chem. 2009, 284, 31817-31826.

[60] Y. Park, W. Vennekate, H. Yavuz, J. Preobraschenski, J. M. Hernandez, D. Riedel, P. J. Walla, R. Jahn, J. Biol. Chem. 2014, 289, 16326-16335.

[61] P. I. Hanson, R. Roth, H. Morisaki, R. Jahn, J. E. Heuser, Cell 1997, 90, 523-535.

[62] T. M. Hohl, F. Parlati, C. Wimmer, J. E. Rothman, T. H. Söllner, H. Engelhardt, Mol. Cell 1998, 2, 539-548.

[63] J. Furst, R. Sutton, J. Chen, A. T. Brunger, N. Grigorieff, EMBO J. 2003, 22, 43654374.

[64] K. E. Marz, J. M. Lauer, P. I. Hanson, J. Biol. Chem. 2003, 278, 27000-27008.

[65] S. Takahashi, H. Yamamoto, Z. Matsuda, M. Ogawa, K. Yagyu, T. Taniguchi, T. Miyata, H. Kaba, T. Higuchi, F. Okutani, S. Fujimoto, FEBS Lett. 1995, 368, 455-460.

[66] T. Ishizuka, H. Saisu, S. Odani, T. Abe, Biochem. Biophys. Res. Commun. 1995, 213, 1107-1114.

[67] H. T. McMahon, M. Missler, C. Li, T. C. Südhof, Cell 1995, 83, 111-119.

[68] T. Trimbuch, C. Rosenmund, Nat. Rev. Neurosci. 2016, 17, 118-125.

[69] R. Mohrmann, M. Dhara, D. Bruns, Cell. Mol. Life Sci. 2015, 72, 4221-4235.

[70] K. Reim, H. Wegmeyer, J. H. Brandstätter, M. Xue, C. Rosenmund, T. Dresbach, K. Hofmann, N. Brose, J. Cell Biol. 2005, 169, 669-680.

[71] M. Xue, K. Reim, X. Chen, H.-T. Chao, H. Deng, J. Rizo, N. Brose, C. Rosenmund, Nat. Struct. Mol. Biol. 2007, 14, 949-958.

[72] S. Pabst, M. Margittai, D. Vainius, R. Langen, R. Jahn, D. Fasshauer, J. Biol. Chem. 2002, 277, 7838-7848.

[73] S. Pabst, J. W. Hazzard, W. Antonin, T. C. Südhof, R. Jahn, J. Rizo, D. Fasshauer, J. Biol. Chem. 2000, 275, 19808-19818.

[74] X. Chen, D. R. Tomchick, E. Kovrigin, D. Arac, M. Machius, T. C. Südhof, J. Rizo, Neuron 2002, 33, 397-409. 
[75] N. Brose, Mol. Cells 2008, 25, 7-19.

[76] R. W. Cho, D. Kümmel, F. Li, S. W. Baguley, J. Coleman, J. E. Rothman, J. T. Littleton, PNAS 2014, 111, 10317-10322.

[77] C. G. Giraudo, W. S. Eng, T. J. Melia, J. E. Rothman, Science 2006, 313, 676-680.

[78] C. G. Giraudo, A. Garcia-Diaz, W. S. Eng, A. Yamamoto, T. J. Melia, J. E. Rothman, J. Biol. Chem. 2008, 283, 21211-21219.

[79] C. G. Giraudo, A. Garcia-Diaz, W. S. Eng, Y. Chen, W. A. Hendrickson, T. J. Melia, J. E. Rothman, Science 2009, 323, 512-516.

[80] S. S. Krishnakumar, D. T. Radoff, D. Kümmel, C. G. Giraudo, F. Li, L. Khandan, S. W. Baguley, J. Coleman, K. M. Reinisch, F. Pincet, J. E. Rothman, Nat. Struct. Mol. Biol. 2011, 18, 934-940.

[81] D. Kümmel, S. S. Krishnakumar, D. T. Radoff, F. Li, C. G. Giraudo, F. Pincet, J. E. Rothman, K. M. Reinisch, Nat. Struct. Mol. Biol. 2011, 18, 927-933.

[82] S. S. Krishnakumar, F. Li, J. Coleman, C. M. Schauder, D. Kümmel, F. Pincet, J. E. Rothman, K. M. Reinisch, eLife 2015, 4, e04463.

[83] T. Trimbuch, J. Xu, D. Flaherty, D. R. Tomchick, J. Rizo, C. Rosenmund, eLife 2014, 3, e02391.

[84] M. Xue, T. K. Craig, J. Xu, H.-T. Chao, J. Rizo, C. Rosenmund, Nat. Struct. Mol. Biol. $2010,17,568-575$.

[85] Q. Zhou, Y. Lai, T. Bacaj, M. Zhao, A. Y. Lyubimov, M. Uervirojnangkoorn, O. B. Zeldin, A. S. Brewster, N. K. Sauter, A. E. Cohen, S. M. Soltis, R. Alonso-Mori, M. Chollet, H. T. Lemke, R. A. Pfuetzner, U. B. Choi, W. I. Weis, J. Diao, T. C. Südhof, A. T. Brunger, Nature 2015, 525, 62-67.

[86] I. Fernandez, D. Araç, J. Ubach, S. H. Gerber, O. Shin, Y. Gao, R. G. Anderson, T. C. Südhof, J. Rizo, Neuron 2001, 32, 1057-1069.

[87] D. Araç, X. Chen, H. A. Khant, J. Ubach, S. J. Ludtke, M. Kikkawa, A. E. Johnson, W. Chiu, T. C. Sudhof, J. Rizo, Nat. Struct. Mol. Biol. 2006, 13, 209-217.

[88] A. B. Seven, K. D. Brewer, L. Shi, Q.-X. Jiang, J. Rizo, PNAS 2013, 110, E3243-E3252.

[89] J. Kim, B. Choi, M. Choi, S. Kim, Y. Lai, Y. Shin, N. K. Lee, EMBO J. 2012, 31, 2144-2155.

[90] H.-M. Lee, D. R. Larson, D. S. Lawrence, ACS Chem. Biol. 2009, 4, 409-427. 
[91] M. J. Hansen, W. A. Velema, M. M. Lerch, W. Szymanski, B. L. Feringa, Chem. Soc. Rev. 2015, 44, 3358-3377.

[92] P. Klán, T. Šolomek, C. G. Bochet, A. Blanc, R. Givens, M. Rubina, V. Popik, A. Kostikov, J. Wirz, Chem. Rev. 2013, 113, 119-191.

[93] P. Rathert, T. Raskó, M. Roth, K. Ślaska Kiss, A. Pingoud, A. Kiss, A. Jeltsch, ChemBioChem 2007, 8, 202-207.

[94] A. Kocer, M. Walko, B. L. Feringa, Nat. Protocols 2007, 2, 1426-1437.

[95] S. K. Nandy, R. S. Agnes, D. S. Lawrence, Org. Lett. 2007, 9, 2249-2252.

[96] H. Li, J.-M. Hah, D. S. Lawrence, J. Am. Chem. Soc. 2008, 130, 10474-10475.

[97] M. E. Hahn, J.-P. Pellois, M. Vila-Perelló, T. W. Muir, ChemBioChem 2007, 8, 21002105.

[98] L. Wang, J. Xie, P. G. Schultz, Annu. Rev. Biophys. Biomol. Struct. 2006, 35, 225-249.

[99] C. Brieke, F. Rohrbach, A. Gottschalk, G. Mayer, A. Heckel, Angew. Chem. Int. Ed. 2012, 51, 8446-8476.

[100] J. A. Barltrop, P. J. Plant, P. Schofield, Chem. Commun. 1966, 22, 822-823.

[101] A. Patchornik, B. Amit, R. B. Woodward, J. Am. Chem. Soc. 1970, 92, 6333-6335.

[102] S. B. Cambridge, D. Geissler, S. Keller, B. Cürten, Angew. Chem. Int. Ed. 2006, 45, 2229-2231.

[103] N. Kotzur, B. Briand, M. Beyermann, V. Hagen, J. Am. Chem. Soc. 2009, 131, 1692716931.

[104] T. Eckardt , V. Hagen, B. Schade, R. Schmidt, C. Schweitzer, , J. Bendig, J. Org. Chem. 2002, 67, 703-710.

[105] J. P. Olson, M. R. Banghart, B. L. Sabatini, G. C. R. Ellis-Davies, J. Am. Chem. Soc. 2013, 135, 15948-15954.

[106] J. P. Olson, H.-B. Kwon, K. T. Takasaki, C. Q. Chiu, M. J. Higley, B. L. Sabatini, G. C. R. Ellis-Davies, J. Am. Chem. Soc. 2013, 135, 5954-5957.

[107] A. Specht, J.-S. Thomann, K. Alarcon, W. Wittayanan, D. Ogden, T. Furuta, Y. Kurakawa, M. Goeldner, ChemBioChem 2006, 7, 1690-1695.

[108] S. Kawata, H.-B. Sun, T. Tanaka, K. Takada, Nature 2001, 412, 697-698. 
[109] C. G. Bochet, Tetrahedron Lett. 2000, 41, 6341-6346.

[110] M. Kessler, R. Glatthar, B. Giese, C. G. Bochet, Organic Letters 2003, 5, 1179-1181.

[111] J.-L. Debieux, C. G. Bochet, Chem. Sci. 2012, 3, 405-406.

[112] J. M. Amatrudo, J. P. Olson, H. K. Agarwal, G. C. R. Ellis-Davies, European Journal of Neuroscience 2015, 41, 5-16.

[113] E. Neher, R. Zucker, Neuron 1993, 10, 21-30.

[114] C. Heinemann, R. H. Chow, E. Neher, R. S. Zucker, Biophys. J. 1994, 67, 2546-2557.

[115] J. B. Sørensen, Pflügers Archiv 2004, 448, 347-362.

[116] G. C. R. Ellis-Davies, Chemical Reviews 2008, 108, 1603-1613.

[117] J. H. Kaplan, G. C. Ellis-Davies, PNAS 1988, 85, 6571-6575.

[118] G. H. Kedar, A. S. Munch, J. R. van Weering, J. Malsam, A. Scheutzow, H. de Wit, S. Houy, B. Tawfik, T. H. Söllner, J. B. Sørensen, M. Verhage, J. Neurosci. 2015, 35 , 14172-14182.

[119] A. Groschner, Design and Synthesis of Modified SNARE Proteins with Respect to the $\alpha-S N A P / N S F$ Mediated Disassembly, Cuvillier Verlag, Göttingen, 2011.

[120] J. A. Ernst, A. T. Brunger, J. Biol. Chem. 2003, 278, 8630-8636.

[121] R. B. Merrifield, J. Am. Chem. Soc. 1963, 85, 2149-2154.

[122] R. B. Merrifield, Science 1965, 150, 178-185.

[123] J. Clayden, N. Greeves, S. Warren, W. Peter, Organic Chemistry, $1^{\text {st }}$ ed., Oxford University Press, Oxford, 2001.

[124] Novabiochem Peptide Synthesis 2012/2013, Merck KGaA, Darmstadt, 2012.

[125] R. Behrendt, P. White, J. Offer, J. Pept. Sci. 2016, 22, 4-27.

[126] A. Speicher, T. Klaus, T. Eicher, J. Prakt. Chem. 1998, 340, 581-583.

[127] R. Subirós-Funosas, R. Prohens, R. Barbas, A. El-Faham, F. Albericio, Chem. Eur. J. 2009, 15, 9394-9403.

[128] J. R. Lakowicz, Principles of Fluorescence Spectroscopy, 3 ed., Springer Science+Business Media, LLC, NewYork, 2006.

[129] K. E. van Holde, W. C. Johnson, P. S. Ho, Principles of Physical Biochemistry, $2^{\text {nd }}$ ed., Pearson Prentice Hall, New Jersey, 2006. 
[130] T. Xu, B. Rammner, M. Margittai, A. R. Artalejo, E. Neher, R. Jahn, Cell 1999, 99, 713-722.

[131] H. Robson Marsden, A. Kros, Angew. Chem. Int. Ed. 2010, 49, 2988-3005.

[132] V. K. Rusiecki, S. A. Warne, Bioorg. Med. Chem. Lett. 1993, 3, 707-710.

[133] K. Nakayama, I. Heise, H. Görner, W. Gärtner, Photochem. Photobiol. 2011, 87, 1031-1035.

[134] J. S. Wood, M. Koszelak, J. Liu, D. S. Lawrence, J. Am. Chem. Soc. 1998, 120, $7145-7146$.

[135] M. Wirkner, S. Weis, V. San Miguel, M. Álvarez, R. A. Gropeanu, M. Salierno, A. Sartoris, R. E. Unger, C. J. Kirkpatrick, A. del Campo, ChemBioChem 2011, 12, 2623-2629.

[136] D. Chu, T. Ye, B. Wang, Novel semi-synthetic glycopeptides as antibacterial agents, UK Patent, GB2464617, 2010.

[137] N. Fomina, C. McFearin, M. Sermsakdi, O. Edigin, A. Almutairi, J. Am. Chem. Soc. 2010, 132, 9540-9542.

[138] I. Güell, S. Vilà, L. Micaló, E. Badosa, E. Montesinos, M. Planas, L. Feliu, Eur. J. Org. Chem. 2013, 2013, 4933-4943.

[139] D. Milovanovic, A. Honigmann, S. Koike, F. Göttfert, G. Pähler, M. Junius, S. Müllar, U. Diederichsen, A. Janshoff, H. Grubmüller, H. J. Risselada, C. Eggeling, S. W. Hell, G. van den Bogaart, R. Jahn, Nat. Commun. 2015, 6, 5984-5993.

[140] D. Milovanovic, M. Platen, M. Junius, U. Diederichsen, I. A. T. Schaap, A. Honigmann, R. Jahn, G. van den Bogaart, J. Biol. Chem. 2016, 291, 7868-7876.

[141] D. Milovanovic, Nanoscale organization and dynamics of SNARE proteins in the presynaptic membranes, eDiss, Niedersächsische Staats- und Universitätsbibliothek, GeorgAugust-Universität, Göttingen, 2015.

[142] K. Meyenberg, Synthese und Untersuchung von peptidischen Modellsystemen für SNAREinduzierte Membranfusion, $1^{\text {st }}$ ed., Books on Demand, Norderstedt, 2011.

[143] C. Eggeling, C. Ringemann, R. Medda, G. Schwarzmann, K. Sandhoff, S. Polyakova, V. N. Belov, B. Hein, C. von Middendorff, A. Schonle, S. W. Hell, Nature 2009, 457, $1159-1162$.

[144] G. van den Bogaart, K. Meyenberg, H. J. Risselada, H. Amin, K. I. Willig, B. E. Hubrich, M. Dier, S. W. Hell, H. Grubmüller, U. Diederichsen, R. Jahn, Nature 2011, 479, 552-555. 
[145] M. M. Bradford, Anal. Biochem. 1976, 72, 248-254.

[146] B. J. Olson, J. Markwell, in Current Protocols in Protein Science, John Wiley \& Sons, Inc., 2007, p. 3.4.1.

[147] E. Kaiser, R. Colescott, C. Bossinger, P. Cook, Anal. Biochem. 1970, 34, 595-598. 



\section{List of Figures}

1.1 Model of neuronal SNARE complex located between synaptic vesicle and plasma membrane. . . . . . . . . . . . . . . . . . . . 2

2.1 Synaptic transmission at nerve terminal . . . . . . . . . . . . . . . . . . . . . 6

2.2 Structure of neuronal SNARE proteins and complex . . . . . . . . . . . . . . . . 8

2.3 Structure of $N$-terminal Habc domain of Syntaxin-1A $\ldots \ldots \ldots$

2.4 Structure of Synaptobrevin-2 $(1-116)$ in DPC micelles . . . . . . . . . . . . 11

2.5 Cycle of SNARE-mediated membrane fusion . . . . . . . . . . . . . . . . 13

2.6 Schematic localisation of neuronal membrane fusion machinery at the active zone 15

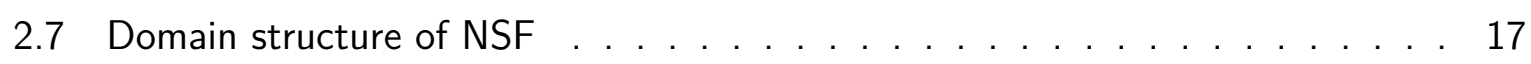

2.8 Full-length NSF in ATP- and ADP-bound state . . . . . . . . . . . . . . . . . . 18

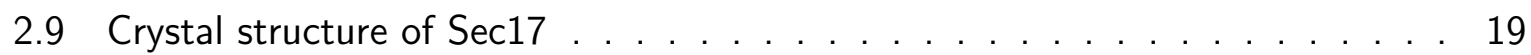

2.10 Timeline of the $20 \mathrm{~S}$ complex structures $\ldots \ldots \ldots$. . . . . . . . . 20

2.11 Interaction between $\mathrm{N}$ domains and $\alpha$-SNAP . . . . . . . . . . . . . . . 22

2.12 Interactions between $\alpha$-SNAPs and SNARE complex . . . . . . . . . . . . . . 23

2.13 Suggested mechanism of NSF/ $\alpha$-SNAP-mediated SNARE complex disassembly 24

2.14 Domain structure of Complexin I $(\mathrm{Cpx} \mathrm{I}) \ldots \ldots$. . . . . . . . . . 26

2.15 Crystal structure of the Complexin/SNARE complex . . . . . . . . . . . . 27

2.16 Sequence and crystal structure of 'superclamp' Complexin/SNARE complex . . 28

2.17 Hypothesised model for the facilitatory function of Complexin's $N$-terminal domain . . . . . . . . . . . . . . . . . . . 30

2.18 Structure of Synaptotagmin-1 (Syt-1) and hypothesised model for cooperation between SNARE complex, Syt-1 and Complexin . . . . . . . . . . . . . . . 32

2.19 Crystal structure of Syt-1-SNARE complex and suggested model . . . . . . . . 33

3.1 Emission spectra of general-purpose light sources . . . . . . . . . . . . . . . . . . 39

3.2 Structures of the 0 -nitrobenzyl moiety used as PPGs . . . . . . . . . . . . . . 40

3.3 Structures of coumarin-4-ylmethyl (CM) derivatives used as PPGs . . . . . . . . 41

3.4 Comparison of single- and two-photon excitation . . . . . . . . . . . . . . 43

3.5 Principle of wavelength-selective uncaging . . . . . . . . . . . . . . . . . . . 44

3.6 Example of solution-phase peptide synthesis including wavelength-selective PPGs. 45

3.7 Examples of photocleavable $\mathrm{Ca}^{2+}$ chelators . . . . . . . . . . . . . 46 
4.1 Structure of $\alpha$-SNAP and modified residues of SNARE complex .

4.2 Synaptobrevin-2 sequence with modifications and structure of unnatural amino acid building block . . . . . . . . . . . . . . . . . . . . . . . . . . . . . . . . . 49

4.3 Synthesis cycle of solid-phase peptide synthesis (SPPS) . . . . . . . . . . . . 50

4.4 Summary of previous results regarding SNARE complex assembly and disassembly properties . . . . . . . . . . . . . . . . . . . . . 52

4.5 Synthesis scheme Fmoc-Asn(Ad)-OH . . . . . . . . . . . . . . . . . . . . . . 52

4.6 Sequences of adamantyl-modified Synaptobrevin-2 derivatives . . . . . . . . . 53

4.7 Experimental setup for fluorescence anisotropy measurements . . . . . . . . . 54

4.8 Schematics of different methods for fluorescence anisotropy measurements . . 56

4.9 Fractions obtained by co-floatation assays analysed by SDS-PAGE . . . . . . . 58

4.10 SNARE assembly experiments with OG-labelled $\Delta$ N complex. . . . . . . . . . . 60

4.11 Disassembly reaction of SNARE complex containing synthesised Syb-2 (28 - 84)

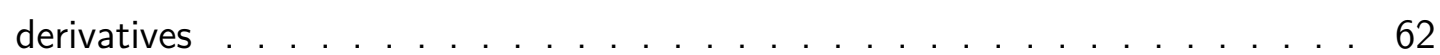

4.12 Complexin binding studies by fluorescence anisotropy as a proof of principle . . 64

4.13 Complexin binding studies of SNARE complexes containing Syb-2 (28 - 84) wild-type (6) . . . . . . . . . . . . . . . . . . . . . . . . . . 66

4.14 Complexin binding studies of SNARE complexes containing Syb-2 (28 - 84)

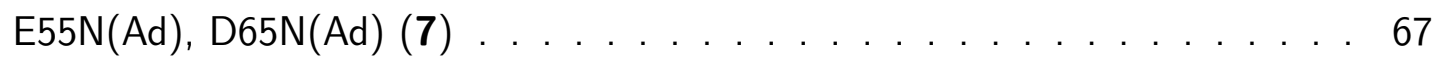

4.15 Complexin binding studies of SNARE complexes containing Syb-2 (28 - 84) Q58N(Ad) (10) . . . . . . . . . . . . . . . . . . . 68

4.16 Validation of complex formation and Complexin binding studies of SNARE complexes containing Syb-2 (28 - 84) Q58N(Ad) (10) . . . . . . . . . . . . . 69

4.17 Validation of complex formation and Complexin binding studies of binary complexes 70

4.18 An overview of performed Complexin binding experiments . . . . . . . . . . . 71

5.1 Overview of investigated Syb-2 truncated sequences regarding SNARE assembly properties. . . . . . . . . . . . . . . . . . . . . . . 77

5.2 Summary of Synaptobrevin-2 point mutation studies . . . . . . . . . . . . . 78

5.3 Helical-wheel of parallel coiled coil dimer . . . . . . . . . . . . . . . . . . . . 80

5.4 Suitable regions of amino acid residues for modification of Synaptobrevin-2 $(28-84)$ SNARE motif . . . . . . . . . . . . . . . . . . . . 80

5.5 Caged amino acid building blocks needed for synthesis of soluble caged Syb-2 . 81

5.6 Synthesis procedure of the caged lysine building block Fmoc-Lys(Nvoc)-OH (16) 82

5.7 Synthesis procedure of the caged arginine building block Fmoc-Arg(Nvoc)-OH (21)

5.8 Synthesis procedure of the caged aspartic acid building block Fmoc-Asp(DMNPB)$\mathrm{OH}(27)$ 
5.9 Overview of synthesised caged test peptides $\mathbf{2 8}-\mathbf{3 1}$ and non-caged peptides 32 and 33

5.10 Test reactions investigating stability of Fmoc-Arg(Nvoc)-OH (21) under standard Fmoc-SPPS conditions . . . . . . . . . . . . . . . . . . . 85

5.11 UV/vis absorption spectra of caged test peptides $29-31 \ldots$. . . . . . . . 86

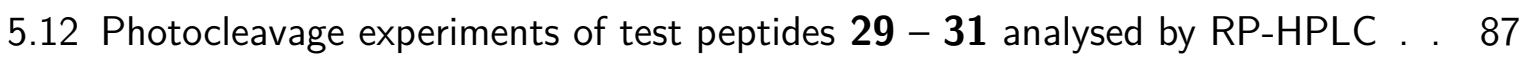

5.13 Proportion of caged peptide or corresponding photocleavage experiments . . . 88

5.14 Synthesis strategy of soluble caged Synaptobrevin-2 (36) . . . . . . . . . . . . 89

5.15 Photocleavage experiments of soluble caged Syb-2 (28 - 84) K52K(Nvoc), D57D(DMNPB), K59K(Nvoc) (36) . . . . . . . . . . . . . . . . . . . . 89

5.16 Binding experiments of $\Delta \mathrm{N}^{\mathrm{OG}}$ complex and soluble caged Syb-2 (28 - 84) K52K(Nvoc), D57D(DMNPB), K59K(Nvoc) (36) . . . . . . . . . . . . 91

6.1 Sequences of Syntaxin TMDs $\mathbf{3 7}-\mathbf{4 0} \ldots \ldots$. . . . . . . . . . . . . . . . . 95

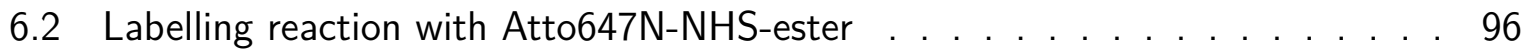

6.3 Labelling reaction with Rhodamine Red-XTM succinimidyl ester . . . . . . . . . 97

6.4 Labelling reaction with Oregon Green ${ }^{\circledR} 488-X$ succinimidylester . . . . . . . . 97

6.5 Sequences of labelled Syntaxin TMDs 41-49 . . . . . . . . . . . . . . . . . . 98

6.6 Sequences of synthesised unlabelled and labelled VAMP TMDs $50-57$. . . . 99 



\section{Acknowledgement/Danksagung}

Besonders danken möchte ich Prof. Dr. Ulf Diederichsen für die Möglichkeit an einer sehr interessanten und äußerst kollaborativen Themenstellung innerhalb seiner Gruppe arbeiten zu dürfen, für seine Unterstützung und Betreuung sowie die gewährte wissenschaftliche Freiheit.

Prof. Dr. Reinhard JAhn danke ich für die freundliche Übernahme des Korreferats, die Betreuung im Rahmen meines Thesis Committees sowie für die Möglichkeit Experimente in seinem Labor durchführen zu können.

Weiterer Dank geht an Prof. Dr. Claudia Steinem für Ihre Ratschläge und Anregungen während der Thesis Committee Meetings.

Prof. Dr. Claudia Höbartner, Dr. Franziska Thomas und Dr. Alexander Breder möchte ich herzlich für die Teilnahme an meiner Prüfungskommission danken.

Herzlicher Dank geht auch an Dr. Halenur Yavuz und Dr. Dragomir Milovanovic aus der Arbeitsgruppe von Prof. Dr. ReINHARD JAHN für die tolle Kooperation im Rahmen des SFB 803 und stete Hilfs- und Diskussionsbereitschaft.

Dem gesamten Team der Massenabteilung - Dr. Holm Frauendorf, Gabriele Krökel und GYÖRGYI SOMmER-UDVARNOKI - möchte ich vielmals danken für die gewissenhafte Anfertigung zahlloser Massenspektren.

Ebenso gilt mein Dank der NMR-Abteilung - Dipl.-Chem. Reinhard MaChineK, Dr. MiChaEL John, Petra Eckhardt, Christiane Siebert, Martin Weitemeyer und Ralf SCHÖNE - für die Anfertigung meiner NMR-Spektren.

Weiterer Dank geht an AOIFE NEviLle für ihre immer währende und freundliche Hilfsbereitschaft. Desweiteren möchte ich AntJe Erdmann, Frauke Bergmann und Tina Trost vom PBCS office danken, dass sie bei Problemen immer mit Rat und Tat da waren.

Meinen ehemaligen Laborkollegen Dr. Selda Kabatas, Dr. Diana Petersen und Anna VERA LÜBBEn möchte ich herzlich danken für die tolle Atmosphäre im Labor 106. Girl's 
power! ;) Dem gesamten AKD danke ich für die gute Atmosphäre und stets vorhandene Hilfsbereitschaft. Besonders der "Kaffeeraum-Gang" - Brigitte Worbs, Christine Hansen, Swantje Nawratil, Janine Wegner und Julia Schneider - möchte ich für die schönen und unterhaltsamen Mittagspausen danken. Außerdem herzlichen Dank an Dr. URSUlA Kotyrba, Barbara Hubrich und Daniel Frank für das gemeinsame "Durchleiden" von HPLC Problemen. Außerdem danke ich Dr. HARITA RAO für die vielen schönen und aufmunterden Gespräche.

Mein besonderer Dank gilt Dr. Selda Kabatas, Dr. Harita Rao, Barbara Hubrich, Christine Hansen, Swantje Nawratil, Julia Schneider, Julia Graf und JanHENRIK QUAST für das Korrekturlesen dieser Arbeit.

Meiner ganzen Familie, vor allem meinen Eltern, möchte ich danken für ihre grenzenlose Unterstützung und ihr Verständnis während meines gesamten Studiums. Danke, dass Ihr mir dieses Studium ermöglicht habt und mir immer mit Rat und Tat zur Seite standet. Danke, für Eure vielen Ermutigungen, ohne die diese Arbeit nicht möglich gewesen wäre.

Mein allergrößter Dank gilt der Liebe meines Lebens, MARCus Junius: Ohne dich hätte ich das alles nicht geschafft. Deine grenzenlose Liebe, Geduld und Heiterkeit, besonders während der "heißen" Phase, haben das alles erst möglich gemacht. Danke für die vielen gemeinsamen Stunden, in denen du mir (geduldig) zugehört hast, mich wieder aufgebaut und zum Lachen gebracht hast. Ich danke dir auch herzlich dafür, dass du mich regelmäßig von der Chemie "befreit" hast. 


\section{Declaration of Authorship}

Herewith I declare, that I prepared the PhD Thesis "Synthesis and Analysis of Modified SNARE Proteins with Respect to Assembly and Disassembly of the SNARE Complex" on my own and with no other sources and aids than quoted.

Göttingen, 29.06.2016

Meike Junius 